\title{
Coordinate-targeted optical nanoscopy: molecular photobleaching and imaging of heterostructured nanowires
}

\author{
DisSERTATION \\ FOR THE AWARD OF THE DEGREE \\ 'DOCTOR RERUM NATURALIUM' (Dr.RER.NAT.) \\ OF THE GeORG-August-Universität GötTINGEN \\ WITHIN THE DOCTORAL PROGRAM PRoPhys
}

of the Georg-August University School of Science (GAUSS)

PUt FORWARD BY

JoANNA ORACZ

BORn In Katowice, Poland 
Thesis Committee

Prof. Dr. Dr. h.c. mult. Stefan W. Hell

(Max Planck Institute for Biophysical Chemistry, Göttingen, Germany)

Prof. Dr. Tim Salditt

(Institute for X-ray Physics, University of Göttingen, Germany)

Prof. Dr. hab. Czesław Radzewicz

(Institute of Experimental Physics, University of Warsaw, Poland)

Members of the Examination Board

Reviewer: Prof. Dr. Dr. h.c. mult. Stefan W. Hell

(Max Planck Institute for Biophysical Chemistry, Göttingen, Germany)

Reviewer: Prof. Dr. Tim Salditt

(Institute for X-ray Physics, University of Göttingen, Germany)

Further MEMBers of the ExAmination BoARd

PD Dr. Alexander Egner

(Department of Optical Nanoscopy, Laser Laboratory Göttingen, Germany)

Prof. Dr. Claus Ropers

(IV. Physical Institute, University of Göttingen, Germany)

Date of the oral examination: 8th March 2018 
Here I declare that my doctoral thesis entitled 'Coordinate-targeted optical nanoscopy: molecular photobleaching and imaging of heterostructured nanowires' has been written independently with no other sources and aids than quoted.

Göttingen, January 2018

Joanna Oracz 



\section{Abstract}

Super-resolution far-field fluorescence microscopy (optical nanoscopy) is a mature set of methods which enable visualization of the nanometer-scale distribution of objects such as organic molecules, photoswitchable proteins, point-like defects in the diamond lattice, upconversion nanoparticles, semiconductor quantum dots, etc. Utilization of internal emitter states in the imaging scheme has made it possible to create contrast at the nanoscale in conventional lens-based optical imaging systems. Spatial resolutions down to the single nanometer scale have been reported in some cases. Nonetheless, routine biological experiments, which employ the molecular probes in physiological conditions, are hampered by photodestructive chemical reactions of fluorophores (photobleaching) which limit signal levels and the attainable resolution to $\sim 20-50 \mathrm{~nm}$. Substantial effort has been devoted to both finding more photostable markers and minimizing the light-induced damage by novel experimental strategies.

This thesis is concerned with coordinate-targeted super-resolution microscopy techniques applied to imaging of common molecular probes and semiconductor heterostructured nanowires at room temperature. These techniques have already demonstrated single nanometer resolution in far-field optical microscopy for ultra-stable emitters: namely color-defects in the diamond lattice. Similar resolution levels have not been reported for standard fluorophores imaged at room temperature. Therefore, the question arises how to increase the imaging capabilities of super-resolution microscopy under biologically relevant conditions.

The first aim of this thesis was to gain new insights into the photobleaching of organic dyes under photon fluxes typically applied in stimulated emission depletion (STED) microscopy. The impact of STED-light photons on the photobleaching of several organic molecules was studied with the goal to identify optimal imaging conditions. To this end, an optical system and experimental strategy were developed to systematically assess the key parameters in STED microscopy: transient de-excitation, irreversible photobleaching and STED-light-induced fluorescence resulting from undesirable excitation events caused by absorption of the STED-light photons. These parameters determine to what extent the STED concept works in practice with a specific dye. We varied the STED pulse duration from $0.13 \mathrm{ps}$ to $500 \mathrm{ps}$ and the time-averaged STED power up to $200 \mathrm{~mW}$ at $80 \mathrm{MHz}$ repetition rate at the popular wavelength of $750 \mathrm{~nm}$, examining common fluorescent compounds (ATTO590, STAR580, ATTO647N, STAR635P) in bulk experiments in thiodiglycol. The magnitude of photobleaching was different for different dyes. In general, two characteristic photobleaching regimes at a given STED pulse energy were found: intensity-dependent (high-order) and intensity-independent (low-order) bleaching. Surprisingly, for ATTO647N we observed a single effective photobleaching scaling over a wide range of STED peak powers $(\sim 0.1-200 \mathrm{~W})$. Based on this observation, we developed an intuitive model for this dye which provides a quantitative prediction of the influence of STED-light photons on the resolution and bleaching. We inferred the spatial distribution of photobleaching probability, the role of detection time gating and the impact of residual STED intensity at the targeted coordinate on the resulting image at different STED pulse energies. The dominant bleaching mechanism determines the optimal STED pulse duration to acquire a super-resolved image with minimal photodamage of the marker. High-order photobleaching can be efficiently reduced by increasing STED pulse duration up 
to roughly the fluorescence lifetime. For low-order photobleaching, chemical triplet quenchers and optical strategies allowing dark-state relaxation hold more promise. Overall, this is the first systematic study of molecular photobleaching in STED microscopy, aimed at finding the optimal optical conditions which minimize STED-light-induced damage.

The second project within this thesis investigated the inherent photoluminescence of heterostructured gallium phosphide-gallium indium phosphide (GaP-GaInP) nanowires (NWs) to improve the resolution of far-field optical microscopy of these emitters. Due to their small diameter $(<100 \mathrm{~nm})$ but significantly larger length, and their tunable electro-optical properties, semiconductor nanowires are gaining interest in intra- and extracellular biological research. They hold potential as local probes of, for example, electric field or forces. Many of these applications require precise localization and identification of NWs featuring different geometries and surface coatings in a scattering biological environment. Traditional fluorescence microscopy, while suitable for nanowires and live-cell imaging, is hampered by limited spatial resolution. We addressed this issue and found that ground state depletion (GSD) microscopy can resolve heterostructured nanowires with a 5-fold resolution enhancement over confocal microscopy. This resolution improvement allowed us to image nanowires with diameters of 20-80 nm characterized by different geometries of photoluminescent GaInP segments of lengths $50-200 \mathrm{~nm}$ spaced by $50-150 \mathrm{~nm}$. The influence of the GaInP segment sizes and positions within a single NW on the GSD image contrast is discussed in detail. The relative simplicity of this method and its moderate laser power requirements make it relevant for further biological studies. 


\section{Published content and contributions}

This thesis comprises the following publications:

1) Photobleaching in STED nanoscopy and its dependence on the photon flux applied for reversible silencing of the fluorophore

Joanna Oracz, Volker Westphal, Czesław Radzewicz, Steffen J. Sahl and Stefan W. Hell

Scientific Reports 7, 11354 (2017)

Reprinted on pages 51-64.

I built the experimental setup, designed and performed all measurements, analyzed the data and wrote numerical simulations with input from all co-authors. I wrote the first draft of the manuscript. The final version was written together with Dr. Steffen J. Sahl and discussed with all co-authors.

2) Ground state depletion nanoscopy resolves semiconductor nanowire barcode segments at room temperature

Joanna Oracz, Karl Adolfsson, Volker Westphal, Czesław Radzewicz, Magnus T. Borgström, Steffen J. Sahl, Christelle N. Prinz, and Stefan W. Hell

Nano Letters 17, 2652-2659 (2017)

Reprinted on pages 85-92.

I built the experimental setup, designed and conducted GSD nanoscopy imaging of nanowires, and performed numerical simulations. I wrote the first draft of the manuscript. The final manuscript was written together with Dr. Steffen J. Sahl and contributions of all co-authors. 



\section{Contents}

Abstract $\quad$ v

$\begin{array}{ll}\text { Published content and contributions } & \text { vii }\end{array}$

$\begin{array}{ll}\text { Table of contents } & \text { ix }\end{array}$

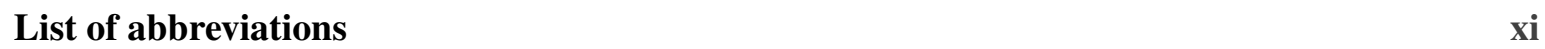

1 Introduction 1

2 Background $\quad 5$

2.1 Advances in far-field optical nanoscopy . . . . . . . . . . . . . . . . . 5

2.2 STED nanoscopy . . . . . . . . . . . . . . . . . . . . . . 10

2.2.1 Diffraction-limited focal spots . . . . . . . . . . . . 13

2.2.2 Resolution scaling with de-excitation photon flux . . . . . . . . . . 15

2.2.3 Photobleaching of molecular probes . . . . . . . . . . . . . 17

2.3 GSD nanoscopy with emitters resistant to photobleaching . . . . . . . . . . . . . 22

2.3.1 Quantum emitters alternative to molecular probes . . . . . . . . . . 22

2.3.2 GaP-GaInP heterostructured nanowires . . . . . . . . . . . . 25

2.3.3 Principles of GSD microscopy . . . . . . . . . . . . . . 27

2.3.4 Resolution scaling with excitation photon flux . . . . . . . . . . 30

2.3.5 Influence of the excitation pulse duration on the GSD image . . . . . . . 31

2.3.6 Restoration of emitter distributions from the GSD image . . . . . . . . . 32

3 Concluding discussion $\quad 35$

$\begin{array}{lr}\text { Bibliography } & 39\end{array}$

4 Photobleaching in STED nanoscopy and its dependence on the photon flux applied for reversible silencing of the fluorophore

5 Ground state depletion nanoscopy resolves semiconductor nanowire barcode segments at room temperature 
Curriculum vitae 


\section{List of abbreviations}

\begin{tabular}{|c|c|}
\hline 1PE & one-photon excitation \\
\hline 2PE & two-photon excitation \\
\hline 3D & three-dimensional \\
\hline APD & avalanche photodiode \\
\hline CW & continuous wave \\
\hline DM & dichroic mirror \\
\hline DOF & depth of field \\
\hline DyMIN & dynamic intensity minimum \\
\hline ESA & excited state absorption \\
\hline FOV & field of view \\
\hline FRET & Förster resonance energy transfer \\
\hline FWHM & full width at half maximum \\
\hline GFP & green fluorescent protein \\
\hline GSD & ground state depletion \\
\hline gSTED & time-gated STED \\
\hline HPP & helical phase plate \\
\hline ISC & intersystem crossing \\
\hline MINFIELD & minimal scanning field \\
\hline MINFLUX & minimal photon flux \\
\hline NV & nitrogen-vacancy \\
\hline NW & nanowire \\
\hline
\end{tabular}


Obj objective lens

PA-GFP photoactivatable GFP

PALM photo-activated localization microscopy

PSF point spread function

QD quantum dot

RESCue reduction of state transition cycles

RESOLFT reversible saturable optical linear fluorescence transitions

SLM spatial light modulator

STED stimulated emission depletion

STORM stochastic optical reconstruction microscopy

T-Rex triplet relaxation

TCSPC time-correlated single photon counting

TDE thiodiglycol 


\section{Chapter 1}

\section{Introduction}

The ability to observe biological specimens at different length scales has led to numerous discoveries throughout the history of natural sciences. One good example is Robert Hooke, a 17th-century English natural philosopher who, by using the first compound-lens microscope, distinguished basic units in plants which he called cells ${ }^{1}$. Since then, far-field optical microscopy has become one of the most powerful techniques in the natural sciences largely due to its non-invasive nature. Unfortunately, many biological objects are highly transparent to visible light and difficult to image with transmitted or scattered photons. For this reason, cellular imaging currently relies heavily on fluorescence microscopy. This technique is based on the specific labeling of targets by using small molecules, which absorb visible photons and emit light of longer wavelength in a process called fluorescence. Certain advantages, such as high specificity, high contrast, multiplexing and real-time 3D visualization make fluorescence microscopy irreplaceable in the imaging of living systems. Despite these benefits, this powerful technique has been for a long time limited in resolution by the diffraction of visible light ${ }^{2}$ to roughly $200 \mathrm{~nm}$. Many biologically relevant entities, such as viruses or protein complexes are considerably smaller than this so-called diffraction limit. Due to the lack of alternatives, scientists have used complementary techniques which can provide even atomic resolution, such as, for instance, electron microscopy. However, these other methods often limit clear conclusions in the biological context due to the requirement to investigate the process of interest under specific conditions: near the surface of glass or air, or after a harsh sample preparation. Not surprisingly, many efforts were made to overcome the diffraction barrier in optical microscopy. Slight resolution improvements were realized, for example, by the confocal detection (which mainly improves background suppression) or two-photon excitation schemes (leading to a minor improvement in the lateral resolution by a factor of $\sim \sqrt{2}$ in comparison with one-photon excitation at a given wavelength). The diffraction limit of fluorescence microscopy was finally overcome by methods called super-resolution microscopy, or simply nanoscopy ${ }^{3,4}$, which were recognized by the award of Nobel Prize in Chemistry in 2014. Resolution enhancements of roughly 10 -fold were demonstrated experimentally by incorporation of at least two intrinsic fluorophores states ('on' $\leftrightarrow$ 'off') in the imaging scheme. Thus, the ability to prepare fluorophores in two discernible states and their properties define resolution in these methods. Superresolution microscopy has been successfully applied to image different types of quantum emitters including organic molecules ${ }^{5,6}$, photoswitchable proteins ${ }^{7,8}$, defects in diamond crystal structure ${ }^{9}$, 
semiconductor quantum dots ${ }^{10-12}$, or upconversion nanoparticles ${ }^{13,14}$. Single-digit nanometer resolutions were first reported for color centers in the diamond lattice at room temperature ${ }^{15}$, and later for organic fluorophores at cryo-temperatures ${ }^{16,17}$. Achieving such results for common fluorescent markers at standard physiological conditions is still challenged by the permanent loss of their ability to emit fluorescence under light exposure, referred to as photobleaching.

In this work, we study the photobleaching of several organic dyes in the super-resolution technique called stimulated emission depletion microscopy (STED) ${ }^{18}$ with the aim to identify the optimal imaging conditions for low bleaching. In particular, we study the effect of the STED pulse duration. In STED microscopy, the flux of photons applied to induce stimulated emission transiently deactivates the marker's ability to emit fluorescence and, in principle, should be harmless to the emitters. However, to achieve high resolution one must apply sufficiently strong de-excitation beam intensities close to the center of the doughnut-shaped STED beam. This results in significant overexposure of the molecular probes to the STED light at the crest of the doughnut beam. Undesirable effects then become problematic as the fluorophores (or neighbor molecules, which then react with the fluorophores) are excited by instantaneous or sequential absorption steps involving photons from the STED beam. These absorption events create populations in higher excited states and result in an increased reactivity of the chemical compounds, as the binding of an excited electron is weaker. Light-induced destruction of fluorescence emitters has been extensively studied in one- and two-photon excitation fluorescence microscopy ${ }^{19-22}$, however systematic studies of photobleaching in STED nanoscopy have been missing. Besides many successful implementations of the STED concept, from sub-nanosecond de-excitation pulses $^{5,23,24}$ up to simple continuous-wave operation ${ }^{25,26}$, the variability of conditions (e.g., spectral distribution of radiation, class of dyes used, labeling density, or host substance) make it difficult to compare various realizations. The previous work on photobleaching in STED microscopy has shown that absorption of STED photons by molecular probes in singlet and triplet systems results in accelerated photodamage ${ }^{27-31}$. However, the impact of the processes involved in photobleaching at different resolution levels was not determined. Here, we investigate in detail how the temporal distribution of photons at a commonly used STED wavelength of $750 \mathrm{~nm}$ affects the photobleaching of several orange- and red-emitting organic fluorophores (ATTO590, STAR580, ATTO647N, STAR635P). The de-excitation photon flux was varied by changing the pulse duration (from subpicosecond pulses to continuous-wave operation) and peak power of STED light (up to $200 \mathrm{~W}$ ) to directly measure transient de-excitation and photobleaching of the fluorescent markers in thiodiglycol (TDE). This study provides an initial step of screening for the optimal parameters in STED microscopy which could minimize bleaching and thus make further resolution enhancement possible.

An interesting alternative to traditional molecular probes which are subject to photobleaching is offered by artificial nanostructures. Their intriguing properties not only allow for efficient imaging, but very often also for novel biological applications. Of particular interest are semiconductor nanostructures, where one can alter optoelectronic properties relatively easily. For example, quantum dots (QDs) are used extensively to visualize biological specimens. Their broad absorption band, narrow and tunable emission spectra resulting from quantum confinement, excellent quantum yield, 
high photostability and biofunctionalization make them useful probes in many applications including multiplexed optical coding, fluorescence resonance energy transfer or high-throughput screening ${ }^{32-35}$. One-dimensional nanostructures, such as nanowires (NW) or nanotubes, allow for measurements of electrical activity ${ }^{36,37}$ or cellular forces ${ }^{38,39}$. Despite the clear potential of artificial nanostructures in sensing applications, their use within living specimens is still controversial due to their poorly characterized cell permeability and toxicity. The interactions at the nanoscale are still not well understood and require further studies. Reproducibility and high levels of control over epitaxial growth of NWs make them perfect model structures in these studies. Previously, NWs were studied in biological contexts by traditional optical microscopy ${ }^{40-43}$. To improve the contrast in scattering biological samples, NWs were imaged with common fluorescent dyes either by direct labeling of their surface or by spatial exclusion of the surrounding fluorescent dye. These experiments were hampered by limited resolution which did not allow for precise localization of the nanostructures with respect to the cell compartments, and by photobleaching of the fluorescent dyes, which limited long-term studies. It has been proposed that photobleaching issues might be overcome by using as a contrast mechanism the inherent photoluminescence of the semiconductor $\mathrm{NWs}^{44}$. The designed heterostructured pattern, created by alternation of non-luminescent $\mathrm{GaP}$ and luminescent GaInP segments ( 'barcodes') within a single NW, allowed for identification of multiple NWs with different geometries and surface coatings in a tissue. Some barcode-structured NWs were not resolvable using a confocal microscope ${ }^{44}$. The success of nanoscopy methods demonstrated for other emitters encouraged us to test if it is possible to increase resolution for the extended semiconductor photoluminescent segments of the NWs. The most common optical nanoscopy techniques as $\mathrm{STED}^{18}$ or PALM $^{7,8} / \mathrm{STORM}^{6}$ are difficult to apply directly to the heterostructured nanowires, as their optical properties differ from commonly used point-like molecular probes. In this work, we study the photoluminescence of $\mathrm{GaP}-\mathrm{GaInP}$ nanowires to improve the resolution of far-field optical microscopy in this system.

\section{Structure of the thesis}

Chapter 2 contains an introduction to the optical nanoscopy methods, highlighting some of the current developments and challenges in this dynamically growing research area. Nanoscopy techniques relevant to the content of the thesis (STED nanoscopy and GSD nanoscopy) are described in more detail, to allow the reader to follow the specific topics covered in the further parts of the thesis. Chapter 3 summarizes the results and provides a general discussion. The following Chapter 4 comprises the results on molecular photobleaching in STED microscopy, as published in Scientific Reports. Finally, Chapter 5 contains super-resolution imaging of heterostructured GaP-GaInP nanowires using GSD nanoscopy; work which was published in Nano Letters. 



\section{Chapter 2}

\section{Background}

\subsection{Advances in far-field optical nanoscopy}

The early $21^{\text {st }}$ century has witnessed a fundamental change in our perception of lens-based optical microscopy. An almost 10-fold resolution enhancement has been achieved over the last two decades for imaging with traditional fluorescent markers. The increased ability to distinguish closely spaced objects emerged from the incorporation of intrinsic properties of the molecular probes in the imaging scheme. The utilization of at least two emitter states, typically a fluorescent 'on' and a non-fluorescent 'off' state, makes it possible to push the resolution, limited by diffraction to $\sim 200 \mathrm{~nm}$ for visible light, down to $\sim 20-50 \mathrm{~nm}$ in biological samples. Generally, super-resolution techniques can be divided into two categories described briefly below: the first group separates features by the molecular states at targeted position in space (coordinate-targeted methods), whereas the second group establishes the state difference at the single-molecule level and at random positions (coordinate-stochastic methods).

In coordinate-targeted methods (e.g., STED ${ }^{18}$, GSD $^{45}$, RESOLFT ${ }^{46}$ ), differentiation of molecular 'on' and 'off' states within the extended diffraction-limited focal volume is realized by a spatially shaped transition beam. This beam should confine the molecular state difference to a possibly small region. In fact, these methods scale down the point spread function (PSF) of the far-field scanning optical microscope. The resolution improvement is determined by the efficiency of the 'on' $\leftrightarrow$ 'off' transition. For a linear optical transition, the spatial resolution $\Delta d_{\mathrm{eff}}$ scales roughly inversely proportionally to the square root of the transition beam intensity $I\left(\Delta d_{\mathrm{eff}} \propto I^{-1 / 2}\right)$. The most intuitive switching mechanism is realized in stimulated emission depletion microscopy (STED ${ }^{18}$ ). To transiently silence the fluorescence at the outer region of the diffraction-limited excitation spot, a doughnut-shaped de-excitation beam (transition beam) is applied. Fluorescence is allowed only in the close vicinity of the coordinate targeted by the minimum of the STED beam which, ideally, features zero intensity. In the outer regions molecules are transferred to the ground state by stimulated emission, before fluorescence can occur. The STED intensity (more precisely, the efficiency of stimulated emission) determines the effective fluorescent volume. As an image is created by raster scanning of the region of interest, the diminished fluorescent volume corresponds to the increase in optical resolution.

In stochastic methods (e.g., PALM $^{7}$, FPALM $^{8}$, STORM $^{6}$, PAINT ${ }^{47}$, GSDIM $^{48}$ ), the separation of 
emitters in close vicinity occurs also by 'on' $\leftrightarrow$ 'off' discrimination, but somewhat differently: at a given time only a single molecule at a random spatial position within a diffraction-limited area is allowed to emit. The small size of the molecule $(\sim 1 \mathrm{~nm})$ compared to its image spot $(\sim 200 \mathrm{~nm})$ makes it to appear as a point emitter. This image registered on a camera represents the diffraction-limited PSF of the far-field microscope. The intensity distribution in the single-molecule image contains information about the molecular coordinates. These can be determined (e.g., by finding the center of the intensity distribution) with a precision higher than the PSF width itself. The error of localization $\sigma_{r}$ is given by the standard deviation $\sigma_{r}=\sigma_{\mathrm{PSF}} / \sqrt{N}$, where $\sigma_{\mathrm{PSF}}$ is the spatial probability of the photon detection, that is, the standard deviation of the PSF and $N$ is the number of signal photons detected from a single emitter in the 'on'-state. To obtain a truly sub-diffraction image, many individual molecules have to be resolved within the diffraction volume in sequential frames recorded on the camera. In each of them, only an optically resolvable subset of the emitters is activated to the 'on' state, these then emit a number of fluorescence photons to eventually end-up (permanently) in the 'off' state. A simple overlap of the sequential frames results in a blurred image. Nonetheless, the combined localizations of the single molecules contain features separated by distances much closer than the diffraction limit. The (mean) resolution enhancement scales in the same way as the localization precision with the square root of $N\left(\Delta d_{\text {eff }} \propto N^{-1 / 2}\right)$. A single molecule can emit approximately $N=10^{6}$ photons before photobleaching occurs $^{49}$, which in case of a perfect detection system corresponds to a resolution which is three orders of magnitude higher than that of conventional optical microscopy. This very intuitive idea of resolution improvement by localization of a single entity (mentioned by Heisenberg ${ }^{50}$ for scattering of an electron already in 1930) was waiting a few decades for experimental realization in fluorescence microscopy. One of the reasons for this is that registration of the single-molecule signal in the presence of many host molecules requires an exceptional sensitivity for photon-limited emitters, such as the fluorescence markers. This fact demands a high absorption cross-section, fluorescence quantum yield, photostability of the markers as well as detection sensitivity. In biologically relevant conditions, these requirements have to be fulfilled in an aqueous environment at approximately room temperature. Additionally, to acquire super-resolution images, an active control of the emission is necessary. Several attempts of single-molecule localization microscopy were undertaken, including photobleaching 51,52 or blinking ${ }^{53}$ of various emitters, but satisfactory results were not reached until photoswitchable fluorophores were developed (e.g., PA-GFP ${ }^{54,55}$, Dronpa ${ }^{56}, \mathrm{Cy} 5^{6,57}$ ). For instance, the discovery of photoactivatable green fluorescent protein (PA-GFP) was a milestone for photo-activated localization microscopy $\left(\operatorname{PALM}^{7}\right)$. In this method, an optically resolvable subset of photoactivatable proteins is converted to a fluorescent variant ('on') by a weak ultraviolet pulse at the activation wavelength $\lambda_{\text {act }}$. Subsequently, only the activated proteins are able to interact with the excitation light at $\lambda_{\mathrm{exc}}$ and emit fluorescence photons until photobleaching occurs ('off'). The detected image reveals the molecular positions. The sequence is repeated until all markers are photobleached, that is, no fluorescence signal can be detected. Reconstructed positions from the individual camera frames are added together, creating the super-resolved image.

Both aforementioned methods have their strengths and weaknesses. Coordinate-targeted ap- 
proaches provide typically better time resolution and do not require an expertise in photochemistry to obtain a proper kinetic of stochastic switching nor reconstruction of the image, which may produce artifacts. On the other hand, they necessitate higher photon fluxes of implemented beams to acquire the same resolution enhancement as coordinate-stochastic methods. These relatively high light intensities may cause increased photobleaching or phototoxicity in biological samples. Secondly, the experimental setups with a patterned transition beam or even multiple beams are more sophisticated and expensive than the simple wide-field microscopes used for stochastic methods. The choice of the best method depends on the particular imaging needs.

In this context it should be mentioned that the classification of methods presented above is not necessarily clear-cut. Linking the coordinate-targeted emission with stochastic activation (transfer to 'on'-state) of single molecules led to nanoscopy with exceptionally low photon fluxes in an approach called MINFLUX ${ }^{58}$. In this method, single-molecule detection is performed sequentially in time like for the stochastic techniques. The novelty lies in probing an unknown emitter position $x_{\mathrm{em}}$ nearby a minimum $x_{\mathrm{i}}$ of a spatially-shaped excitation beam. In the one-dimensional case, the excitation minimum can be approximated by a parabola $I_{\mathrm{exc}}(x) \propto a\left(x-x_{i}\right)^{2}$. The emitted photon flux $\Phi_{\mathrm{fl}}$ depends on the displacement between the well-defined coordinate of the excitation beam intensity minimum $x_{\mathrm{i}}$ and the unknown molecular position $x_{\mathrm{em}}$. A small shift from the perfect overlap between $x_{\mathrm{i}}$ and $x_{\mathrm{em}}$ (significantly smaller than the beam focal spot size) is reflected by a (small) number of emitted photons. The registered photon flux is proportional to the intensity of the excitation $\Phi_{\mathrm{fl}}(x) \propto a\left(x_{\mathrm{em}}-x_{\mathrm{i}}\right)^{2}$. To obtain the one-dimensional (1D) emitter vector $x_{\text {em }}$, which identifies the emitter position with respect to the beam coordinate $x_{\mathrm{i}}$, at least two consecutive measurements are necessary $i=1,2$. Each of them corresponds to different excitation beam positions and, thus, different numbers of detected photons $n_{1,2} \propto \Phi_{\mathrm{fl}_{1,2}} \propto a\left(x_{\mathrm{em}}-x_{1,2}\right)^{2}$. The $1 \mathrm{D}$ emitter vector can be estimated by $x_{\mathrm{em}}=\frac{x_{2} \sqrt{n_{1}}+x_{1} \sqrt{n_{2}}}{\sqrt{n_{1}}+\sqrt{n_{2}}}$. To obtain the emitter position in two dimensions, at least three consecutive measurements are necessary ${ }^{58}$. Since the coordinates of the excitation beam minimum can be determined a priori with precision $<1 \mathrm{~nm}$, the limitation to molecular position estimation lies in the fluctuations of the detected photon numbers. The photon shot noise determines the minimal detection time for each exposure. In contrast to other super-resolution methods, the applied wavelength no longer enters the expression for the achieved position localization precision (and thus resolution). The MINFLUX concept minimizes the requirements on registered photon numbers to localize single emitters. This technique recently demonstrated state-of-the-art images of molecular probes with one nanometer resolution at room temperature ${ }^{58}$.

Recent developments in nanoscopy techniques focus on further improvements of imaging capabilities. The general goal is to acquire enhanced information about the labeled specimen (3D space and time), with the least possible influence on the observed system. It is actually very challenging from the experimental point of view because the new regime of resolution demands smaller fluorescent labels, sufficient labeling density as required by the Nyquist criterion, aberration-free focal spots possibly over a large depth of field (DOF, that is, far away from the cover slip), minimal photobleaching and phototoxicity, high throughput and a very stable optical system over the measurement time. Particular 
needs can be addressed by establishing new optical strategies, modifying the emitter properties (and their surrounding) or exploring other molecular states to provide the features' separation. In the following, a few examples of important advances in super-resolution microscopy are given.

For point-scanning coordinate-targeted nanoscopy, parallelization was an important step towards speeding up the image acquisition in a large field of view (FOV) $)^{59,60}$. The pixel dwell time in nanoscopy is limited by the 'on' $\leftrightarrow$ 'off' switching kinetics and the necessary number of cycles that a molecule has to undergo to register an acceptable signal-to-noise ratio. For large FOV, the common point scanning approach has a low throughput. Instead of using a single transition beam (which features a single intensity minimum) and a bucket detector, it is advantageous to create an intensity distribution with many minima and register the signal on a camera-based detector from the whole FOV simultaneously. In this case, the need for scanning to separately address subdiffraction features is reduced to within the diffraction-limited area. Parallelization of the image collection has an excellent result for RESOLFT microscopy with photoswitchable proteins, where the photon flux necessary to acquire a substantial resolution gain is relatively moderate ${ }^{3}$. Namely, high-power lasers allow for spreading their energy over a large region, while still maintaining sufficient photon densities nearby the minima of the transition light distribution to accomplish the molecular state difference preparation. Consequently, an image within a $\sim 100 \times 100 \mu \mathrm{m}^{2}$ field with lateral resolution below $100 \mathrm{~nm}$ can be registered in less than a second ${ }^{60}$.

The success of all nanoscopy methods is determined by the photostability of fluorescent markers. This problem is especially severe in STED microscopy, where a majority of the markers' optical transitions 'on' $\leftrightarrow$ 'off' do not contribute to the registered fluorescence signal. The extended exposure of the molecular probes to photons of STED light eventually results in a permanent loss of their ability to fluoresce. To overcome this issue, several strategies based on controlled light exposure have been proposed. It is known from early experiments with dye solutions that one possible photobleaching pathway is mediated by the lowest triplet state. The magnitude of the photodamage assigned to the triplet system can be reduced by preventing a build-up of the lowest triplet state. Triplet relaxation (T-Rex) can be realized for instance by application of low repetition-rate lasers ${ }^{29,30}$. The time interval between consecutive pulses has to be longer than the lifetime of the triplet state. In these conditions, population of the triplet state is minimal and the molecules trapped in this state are not exposed to photons of the following laser pulses. Such an arrangement protects them from promotion to more reactive higher triplet states by absorption of photons from the implemented beams. Reduction of photobleaching increases the maximally applicable STED photon flux and allows for further resolution enhancement. Indeed, the T-Rex concept reported the best resolution to date of $\sim 15-20 \mathrm{~nm}$ in STED microscopy with organic molecules at room temperature ${ }^{29}$. Nonetheless, this method is not popular because it compromises imaging speed significantly. The relatively long lifetime of the molecular triplet state $\left(\sim 10^{-4} \mathrm{sec}\right)$ requires reduction of laser repetition rate from commonly used $80 \mathrm{MHz}$ down to $<1 \mathrm{MHz}$. To maintain the same signal level, the pixel dwell time has to be increased, which is disadvantageous in many applications.

Super-resolution methods based on single-molecule localization are facing other difficulties. For 
instance, the resolution enhancement in the axial direction over the large DOF is particularly challenging. The problem originates from symmetric and elongated shape of the PSF along the objective axis, which does not change significantly over $\sim 100 \mathrm{~nm}$. The common approaches to obtain resolution enhancement in the axial direction are based on introducing defocusing ${ }^{61,62}$ or astigmatism ${ }^{63,64}$ in the detection path of the optical system such that the shape of the single-molecule image in the detector represents the axial coordinate. These methods, while providing super-resolution information in all three dimensions, have limited DOF to approximately the axial PSF size of $\sim 600 \mathrm{~nm}$. The DOF can be slightly improved by multiplane imaging ${ }^{65}$ or adaptive optics strategies ${ }^{66}$. More sophisticated engineering of the PSF can notably increase the DOF. Converting a Gaussian-like PSF to the so-called double helix can more than double the accessible depth in PALM microscopy (to $\sim 2 \mu \mathrm{m})^{67}$. The double helix concentrates the fluorescence light into two spinning lobes over an extended axial range. The actual twist between them registered in the image plane depends on the axial position of the emitter. By application of appropriate estimators, nearly uniform localization precision (i.e. resolution) in lateral and axial directions have been reported ${ }^{67}$.

The possibility to image simultaneously different targets, called multiplexing, is a modality extensively used in fluorescence microscopy. Typical multicolor setups employ several fluorescence filters, which to some extent separate the emission spectral composition of various dyes compatible with the available laser sources. The signals from the individual spectral windows are integrated in the respective bucket detectors and later assigned to different color channels. This approach to create multicolor images suffers from the fact that emission spectra of the dyes often overlap between the color channels. Spectral overlap results in cross-talk which influences the contrast of the final image. The cross-talk can be reduced by a spectrally resolved imaging scheme ${ }^{68}$ demonstrated for stochastic optical reconstruction microscopy (STORM). By applying a dispersive element (e.g., a prism) in the detection path, both the position and the spectra of individual molecules can be detected simultaneously on the camera. Such an experimental design allowed for multicolor imaging of four markers with maxima of spectral distribution separated by only $\sim 10 \mathrm{~nm}$, providing nearly cross-talk-free imaging ${ }^{68}$.

The current advances in optical microscopy provide new insights in biological research ${ }^{69}$. Farfield optical techniques can now access the spatial orientation and dynamics of proteins and protein complexes, providing a better understanding of the intracellular mechanisms. For instance, STED microscopy resolved Bax protein oligomerization, indicating a pore-like release mechanism of proteins from mitochondria to the cytosol ${ }^{70}$. Another example is the organization of actin in the axons of neurons. Actin filaments, among other functions, regulate shape and motility of the cell. STORM fluorescence microscopy, thanks to high spatial resolution and specificity, demonstrated that actin forms regular, isolated rings in axons separated by $\sim 190 \mathrm{~nm}^{71}$. A variety of the cytoskeletal proteins which are contained in the nerve cell hid actin organization in electron microscopy studies. Finally, PALM microscopy visualized hemagglutinin molecules in fibroblasts ${ }^{72}$. The accessible resolution of $\sim 40 \mathrm{~nm}$ and dynamics at $\sim 150 \mathrm{~ms}$ sampling revealed the organization and structure of these proteins, revising the currently existing membrane domain models. 


\subsection{STED nanoscopy}

Stimulated emission depletion (STED) microscopy is the first super-resolution method conceived and described theoretically by Stefan Hell and Jan Wichmann in $1994^{18}$. A few years later, breaking of the diffraction barrier of far-field optical microscopy was demonstrated experimentally. This section serves as a brief introduction to this technique.

The basic scheme of a STED microscope is presented in Fig. 2.1a. The excitation beam (shown in blue) and the STED beam (shown in red) are combined by dichroic mirrors (DMs), in such a way that they overlap in the focal plane of the objective lens $(\mathrm{Obj})$. To ensure uniform interaction of photons with all possible orientations of molecular transition dipoles present in the sample, both beams typically feature a circular polarization. The linear polarization of the incoming beams is transformed into a circular one by a broadband quarter waveplate $(\lambda / 4)$ placed close to the back pupil plane (back aperture) of the objective lens. The diffraction-limited focal spots require laser beams which overfill the objective back pupil plane. Therefore, both beams have diameters of $\sim 5.6 \mathrm{~mm}$. The role of the excitation beam is to excite molecules to a fluorescent state ('on'). A STED beam is implemented to transiently disable the fluorescence ability in the outer region of the excitation spot by stimulated emission ('off'). To accomplish this, the STED beam exhibits an intensity minimum in the middle of the focal spot (see Fig. 2.1c). The STED beam minimum is created by imprinting controlled spatial phase differences on the beam, just before focusing. Phase retardation can be applied by a commercial helical phase plate (HPP) with varying thickness of a polymer layer which corresponds to a $0-2 \pi$ phase shift within the beam (Fig. 2.1a). A perfect zero-intensity minimum is formed only for a certain STED wavelength, as only then the optical path difference corresponds to the desired retardation. The doughnut beam quality depends on the polarization of the STED light. For a tight-focusing objective lens, only one circular polarization (left or right handed, depending on the orientation of the HPP $)^{73}$ results in a good quality of the STED beam, featuring ideally a perfect zero intensity at the center. In many applications the fixed HPP plate is replaced by a more flexible spatial light modulator (SLM), which allows not only for wavelength-dependent doughnut beam shaping, but also for an additional adaptive correction of aberrations caused by transparent or scattering tissue. A different way to generate the doughnut-shaped beam is to modify polarization in a spatially selective manner in the STED beam before focusing ${ }^{74,75}$. Image acquisition occurs as in confocal microscopy by raster scanning of the sample, using a piezoelectric stage or scanning mirrors. Fluorescence (Fig. 2.1a, shown in green) originating at each scanning step is registered with a bucket detector, here a single-photon-sensitive avalanche photodiode (APD). High numerical aperture objectives are infinity-corrected and the image of the fluorescence spot is formed by an additional tube lens (L). In the image plane, a multimode optical fiber (MMF) acts as a confocal pinhole to reject out-of-focus fluorescence and enhance the image contrast.

The molecular states and optical transitions relevant to the image formation in STED microscopy are presented in the Jabłoński diagram in Fig. 2.1b. Possible electronic states are simplified to just two: the ground state ( $S_{0}$, 'off') and the first excited singlet state ( $S_{1}$, 'on'). All molecules are considered to be initially in the ground state. The excitation beam transfers the ground-state molecules to a higher 
a

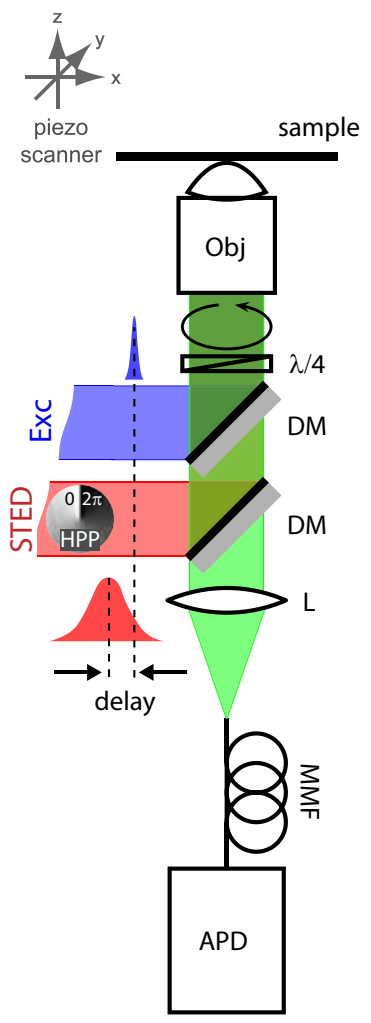

b

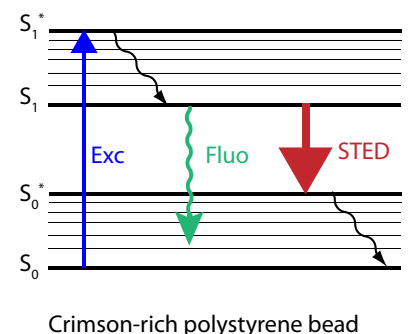

d

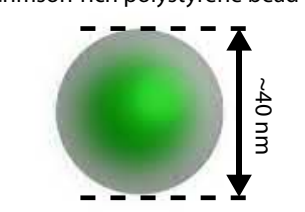

f

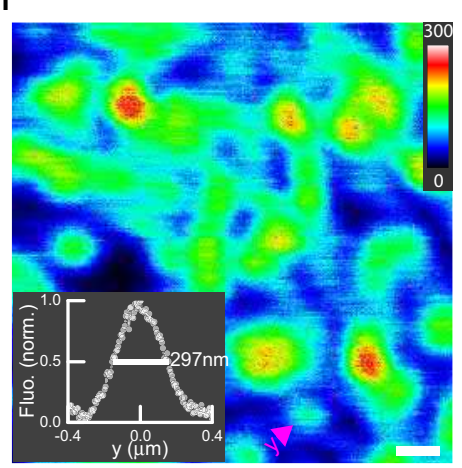

C

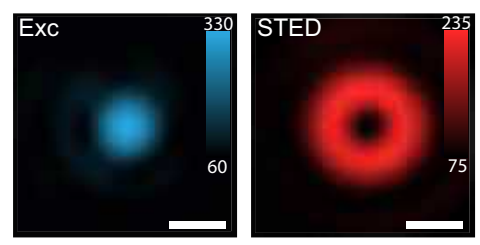

e

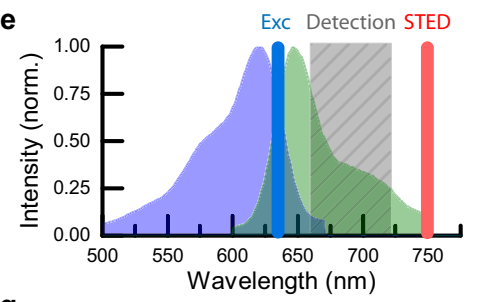

g

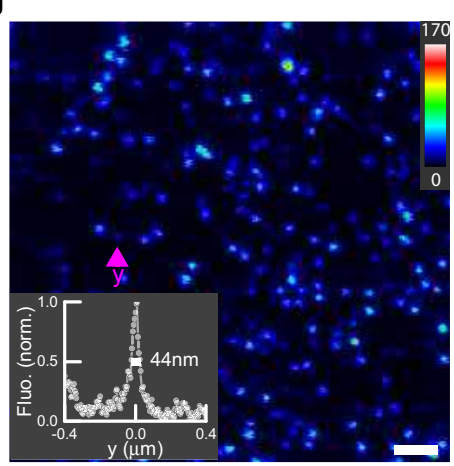

Figure 2.1: STED nanoscopy. (a) Basic scheme of a STED microscope (Obj: objective lens, $\lambda / 4$ : quarterwave plate, DM: dichroic mirror, HPP: helical phase plate, L: tube lens, MMF: multimode fiber, APD: avalanche photodiode). (b) Jabłoński diagram of organic molecule electronic states showing the ground state $\left(S_{0}\right)$, the first excited state $\left(S_{1}\right)$ and the optical transitions of excitation (Exc), spontaneous emission (Fluo) and stimulated emission (STED). Black arrows indicate solvent-related vibrational relaxations. (c) Point-spread functions ( $x y$ sections) of excitation beam (Exc) and STED beam as measured by scattering of an $80 \mathrm{~nm}$ gold bead. The scattered signal was measured separately on a photomultiplier tube (path not shown). (d) Schematic drawing of a $40 \mathrm{~nm}$ diameter crimson fluorescent bead. (e) Spectroscopic properties of crimson dye with selected excitation (Exc), detection and stimulated emission (STED) spectral windows. (f) Example of crimson beads confocal image. The inset shows the intensity profile along the direction indicated by the $y$ arrow. Excitation average power: $10 \mu \mathrm{W}$, excitation central wavelength: $635 \mathrm{~nm}$, excitation pulse duration: $500 \mathrm{fs}$ (FWHM). (g) STED image of the same area as in (f). The inset shows the intensity profile along the direction indicated by the $y$ arrow. STED average power: $300 \mathrm{~mW}$, STED central wavelength: $750 \mathrm{~nm}$, STED pulse duration: $200 \mathrm{ps}$. Excitation properties are the same as in (f). The objective was an immersion oil lens of high numerical aperture (HCX PL APO 100×/1.4NA, Leica). All average powers are measured at the back aperture of the objective lens. Repetition rate: $80 \mathrm{MHz}$. All scale bars: $500 \mathrm{~nm}$.

vibrational level of the first excited singlet state upon absorption of photons $\left(S_{0} \rightarrow S_{1}^{*}\right)$. This process occurs on a femtosecond time scale. Subsequently, within picoseconds, solvent-related nonradiative vibrational relaxation occurs $\left(S_{1}^{*} \rightsquigarrow S_{1}\right)$, followed by fluorescence $\left(S_{1} \rightsquigarrow S_{0}\right)$. The lifetime of the $S_{1}$ state typically equals a few nanoseconds. STED photons deliberately transfer the excited molecules to higher vibrational levels of the ground state by stimulated emission $\left(S_{1} \rightarrow S_{0}^{*}\right)$. The detection spectral 
window has a cut-off below the STED wavelength so that stimulated photons do not contribute to the registered signal (see Fig. 2.1e). By fast vibrational relaxation, $\left(S_{0}^{*} \rightsquigarrow S_{0}\right)$, the molecules return to the initial ground state and the cycle repeats. The role of the STED beam is to transiently disable the markers' ability to emit fluorescence. To do so, stimulated emission has to occur faster than the spontaneous decay. The probability of stimulated emission can be controlled by the number of STED photons acting on the molecules in $S_{1}$ state. In order to obtain a high efficiency of de-excitation, the STED pulse must directly follow the excitation pulse. The dynamics of the involved relaxation processes limit the range of applicable STED pulse durations. To avoid unproductive flipping between singlet states by an intense STED photon flux $\left(S_{0}^{*} \leftrightarrow S_{1}\right)$, the STED pulse has to be longer than the vibrational relaxation of the marker. On the other hand, the STED pulse should act only on the molecules in the first excited singlet state $S_{1}$, which determines the upper limit for the STED pulse duration roughly to the excited state lifetime. This broad range of applicable pulse durations is further constrained by other processes involving STED light, such as undesirable multiphoton or sequential absorption of photons from the STED beam by fluorescent markers. The magnitude of these processes increases with the STED-light intensity, and these effects have different contributions depending on the resolution enhancement required.

The population of molecular states with higher energy (in both singlet and triplet systems) often leads to more pronounced photodamage. Despite the theoretical predictions of the most efficient de-excitation with a few picoseconds STED pulse durations ${ }^{18}$, a significant resolution improvement was not reported with molecular probes until long STED pulses of $\sim 50$ ps were applied ${ }^{23}$. Nowadays, de-excitation is commonly performed with STED pulse durations $\tau_{\text {STED }}$ in the range $200-1000 \mathrm{ps}^{76}$. The STED concept has been also successfully implemented with continuous-wave (CW) de-excitation beams ${ }^{25,26}$. Simpler and lower-cost CW STED setups, however, necessitate a higher average STED power to provide a sufficient de-excitation photon flux over the molecular excited state lifetime. The increased average power results in more pronounced undesired linear processes, such as one-photon excitation of 'hot' ground state molecules (occupying higher vibrational energy levels of $S_{0}$ ) by absorption of STED-light photons. Moreover, as optical switching occurs relatively slowly in CW STED, there is a leakage of fluorescence signal over the STED exposure time called early-fluorescence signal. Early-fluorescence photons partially hamper the contrast and resolution of the registered image. This drawback can be overcome by time-gating of fluorescence detection ${ }^{26,77}$. The signal collection in time-gated STED (gSTED) starts typically $\sim 1 \mathrm{~ns}$ after the excitation pulse. Detection time gating is realized by time-correlated single photon counting (TCSPC) modules, which provide sufficient time resolution. The super-resolution contrast enhancement is achieved at the expense of the registered fluorescence signal.

The quality of the beams and their spatial alignment are determined by measuring the scattered signal from a small object placed in the focal plane. Fig. 2.1c shows the excitation and STED focal spots as measured by scattering of a single $80 \mathrm{~nm}$-diameter gold bead. As the size of the gold bead is smaller than the expected resolution $(\sim 230 \mathrm{~nm})$, the images roughly represent the respective PSFs. Special attention should be paid to the residual intensity in the STED beam minimum. The rule of 
thumb is that high-quality STED imaging requires an intensity minimum below $1 \%$ of the STED doughnut crest value.

An example of the resolution enhancement in STED microscopy is shown for the fluorescent beads dispersed on the cover glass (Fig. 2.1d-g). Fluorescence beads are polymer microspheres heavily loaded with a specific dye. Such shielding protects the organic compounds from potential destructive chemical reactions with the microenvironment. Therefore, fluorescent beads are very bright and photostable, constituting an excellent target to demonstrate the resolution improvement. Here, we imaged crimson fluorescent beads (Thermo Fisher Scientific) with a mean size of $\sim 40 \mathrm{~nm}$ (Fig. 2.1d). The absorption and emission spectra of the crimson dye with the excitation, detection and STED spectral windows are presented in Fig. 2.1e. By application of the excitation beam alone the conventional confocal image is recorded (Fig. 2.1f). Scanning the same area with co-aligned excitation and STED beams results in $\sim 7$ times increased lateral resolution (Fig. 2.1g).

\subsubsection{Diffraction-limited focal spots}

For aberration-free objective lens, the minimal volume of the far-field focal spot is determined by diffraction of the light. The finite beam size in the focal plane directly corresponds to the finite resolution of the raster-scanning optical system. The reason for this is that the signal from all emitters within that volume is collected simultaneously on the detector and smaller features cannot be distinguished in the image. This section concerns the diffraction-limited focal spots of the excitation and de-excitation beams which constitute the best possible starting point for further resolution improvement in STED microscopy.

The so-called diffraction barrier of far-field optical microscopy was first rationalized by Ernst Abbe, who introduced the famous equation indicating a resolution limit ${ }^{2}$

$$
\Delta d \geqslant \frac{\lambda}{2 N A}
$$

where $\Delta d$ is the resolution of the optical microscope in the direction perpendicular to the objective axis (lateral direction), $\lambda$ is the wavelength of light used to create an image and NA is the numerical aperture of the objective lens. The light distribution in the focal plane $I_{\text {focal }}(x, y)$ can be calculated using the Fraunhofer diffraction integral. The exact calculations can be found elsewhere ${ }^{78}$. For a thin lens, the approximate intensity in the focal plane is given by

$$
I_{\text {focal }}(x, y) \propto|\mathscr{F}\{E(x, y) t(x, y)\}|^{2} / \lambda^{2} f^{2}
$$

where $\mathscr{F}$ is the Fourier transform of the electric field $E(x, y)$ just before the focusing lens multiplied by the lens aperture function $t(x, y)$ and $f$ is the lens focal length (Fig. 2.2a). To obtain a minimal focal volume, the Gaussian laser beam has to overfill the back pupil plane of the objective lens. In this case, the electric field $E(x, y)$ is roughly constant within the aperture and the intensity profile in the focal plane corresponds to the Fourier transform of a circular aperture $I_{\text {focal }}(x, y) \propto|\mathscr{F}\{t(x, y)\}|^{2}$ known as the Airy disc (Fig. 2.2c-d). The Airy disc is the smallest focal spot possible created by a circular lens. In the presence of measurement noise, this function is well approximated by a Gaussian 
a
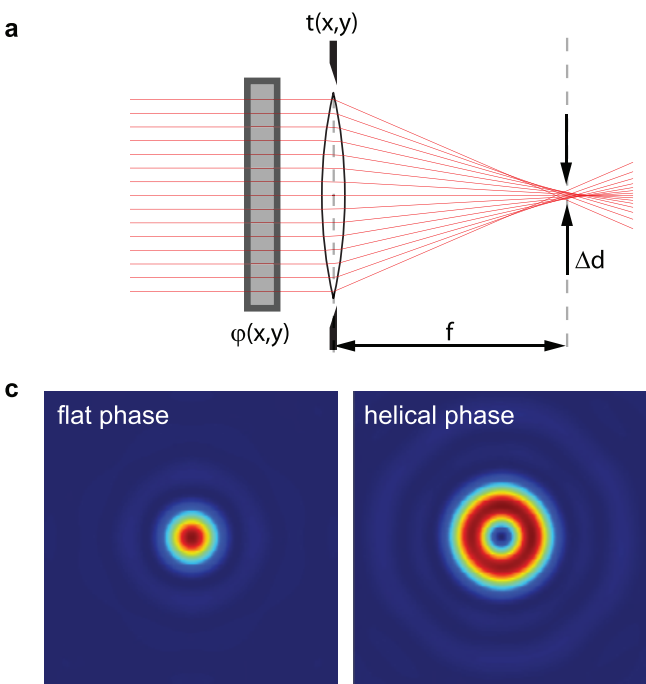

b
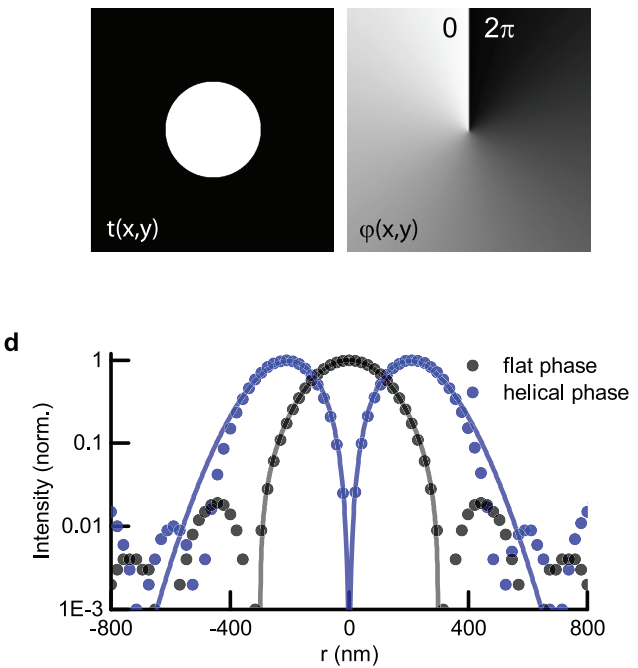

Figure 2.2: Light intensity in the focal plane for flat and helical spatial phase of the laser beam. (a) Schematic drawing of light focusing by a thin lens, where $\varphi(x, y)$ denotes the spatial retardation of the phase plate, $t(x, y)$ is the lens aperture function, $f$ is the lens focal length, and $\Delta d$ is the lateral width of the focal spot. (b) Aperture function of the lens $t(x, y)$ and helical spatial phase $\varphi(x, y)$. (c) Intensity distributions in the focal plane for flat and helical spatial phase of the beam. (d) Intensity profiles (dots) of the images presented in (c) with fitted Gaussian (black line) and Laguerre-Gaussian (blue line) functions. The data in (c,d) was calculated using a scalar diffraction approach.

distribution due to the low intensity of concentric rings $(<2 \%)$ in respect to the bright region in the center (Fig. 2.2d)

$$
I(r)=I_{\mathrm{peak}} e^{-\frac{4 \ln 2 r^{2}}{\Delta d^{2}}}
$$

where $I_{\text {peak }}$ is the maximal beam intensity, $\Delta d$ is the width of the intensity distribution (defined as full width at half maximum, FWHM) in the lateral direction and $r$ is the radial distance. By introducing a spatial phase retarder $\varphi(x, y)$ in front of the lens, the focal spot shape can be altered. For example, the helical spatial phase $\left(\varphi(x, y)=e^{i \theta}\right.$, where $\theta$ is the polar angle in a spherical coordinate system, Fig. 2.2b) can generate a doughnut-shaped intensity profile in the focus (Fig. 2.2c,d). This profile can be approximated by a Laguerre-Gaussian function (Fig. 2.2d) described as

$$
I_{\mathrm{LG}}(r)=I_{\text {peak }}\left(\frac{2 r^{2}}{w_{0}^{2}}\right) e^{1-\frac{2 r^{2}}{w_{0}^{2}}}
$$

where $w_{0}$ is the beam size parameter. For a laser beam operating at the wavelength of $\lambda=750 \mathrm{~nm}$ with a flat spatial phase, the diffraction-limited Gaussian-like focal spot has a diameter $\Delta d \approx 270 \mathrm{~nm}$ (FWHM). By imprinting the helical phase, the same beam focuses to a diffraction-limited Laguerre-Gaussian doughnut-shaped spot with $w_{0} \approx 290 \mathrm{~nm}$. Such a focal profile is characterized by two diameters corresponding to intensities equal half of the crest intensity: the inner dip diameter $\Delta d_{\text {inner }} \approx 190 \mathrm{~nm}$ and the outer diameter $\Delta d_{\text {outer }} \approx 660 \mathrm{~nm}$. The inner diameter of the doughnut-shaped beam is narrower than the corresponding Gaussian-like focus. This fact has been utilized for a minor resolution enhancement in confocal microscopy ${ }^{79}$. 
It is important to note that the calculations presented above are based on the thin lens (smallangle) approximation of scalar diffraction theory. More precise calculations of focal spot intensity distributions for a high numerical aperture objective lens (with an entrance angle $\alpha \approx 72^{\circ}$ ) require including the polarization effects. A vectorial diffraction theory description for high numerical aperture optical systems can be found elsewhere ${ }^{80,81}$.

\subsubsection{Resolution scaling with de-excitation photon flux}

In the ideal case, photons of the STED beam exclusively cause de-excitation of molecules in the first excited singlet state $S_{1}$ (Fig. 2.1a). Changes in the $S_{1}$ population are given by

$$
\frac{d S_{1}}{d t}=-\left(\gamma_{\mathrm{fl}}+\frac{\sigma_{\mathrm{STED}} I_{\mathrm{STED}}}{\hbar \omega}\right) S_{1}
$$

where $S_{1}$ is the population of excited molecules, $\gamma_{\mathrm{fl}}=1 / \tau_{\mathrm{fl}}$ is the fluorescence decay rate with $\tau_{\mathrm{fl}}$ being the fluorescence lifetime, $\sigma_{\text {STED }}$ is the stimulated emission cross-section at the STED wavelength, $I_{\text {STED }}$ is the local intensity of the STED beam and $\hbar \omega$ is the STED photon energy. If we assume a rectangular STED pulse with constant intensity for times $0<t \leq \tau_{\text {STED }}$, we can integrate Equation 2.5 to obtain

$$
S_{1}\left(t, I_{\mathrm{STED}}\right)=S_{1}(0) e^{-\gamma_{\mathrm{ft}} t} e^{-\frac{\sigma_{\mathrm{STED}} I_{\mathrm{STED}}}{\hbar \omega} \tau_{\mathrm{STED}}}, t \geq \tau_{\mathrm{STED}}
$$

where $S_{1}(0)$ is the population of excited molecules at $t=0$ (just after excitation). We can then define the suppression factor of fluorescence emission $\eta\left(I_{\mathrm{STED}}\right)$

$$
\eta\left(I_{\mathrm{STED}}\right)=e^{-\frac{\sigma_{\mathrm{STED}} I_{\mathrm{STED}}}{\hbar \omega}} \tau_{\mathrm{STED}}=e^{-\ln 2 \frac{I_{\mathrm{STED}}}{I_{\mathrm{S}}}}
$$

with a characteristic parameter $I_{\mathrm{s}}$ for which the probability of fluorescence emission is suppressed by a factor of two

$$
I_{\mathrm{S}}=\frac{\ln 2 \hbar \omega}{\sigma_{\mathrm{STED}} \tau_{\mathrm{STED}}} .
$$

The effective PSF $h_{\text {eff }}$ which governs resolution (without confocal detection) is given by

$$
h_{\mathrm{eff}}(r)=h(r) \eta(r)=e^{-\ln 2 \frac{4 r^{2}}{\Delta d^{2}}} e^{-\ln 2 \frac{I_{\mathrm{STED}}(r)}{I_{\mathrm{s}}}}
$$

where $h(r)$ is the diffraction-limited PSF of an excitation focal spot with diameter $\Delta d$. $I_{\mathrm{STED}}(r)$ is described by the Laguerre-Gaussian function (Equation 2.4). Nearby the coordinate targeted by the STED beam minimum, the intensity distribution can be approximated by a parabola $I_{\text {STED }}(r) \approx I_{\text {peak }} \frac{e \cdot 2 r^{2}}{w_{0}^{2}}$ (see Equation 2.4). Therefore, Equation 2.9 simplifies to

$$
h_{\mathrm{eff}}(r) \approx e^{-\ln 2 r^{2}\left(\frac{4}{\Delta d^{2}}+\frac{I_{\mathrm{peak}}}{I_{\mathrm{sat}}} \frac{e}{w_{0}^{2}}\right)} .
$$

The resolution $\Delta d_{\mathrm{eff}}$ can be calculated as the FWHM of $h_{\mathrm{eff}}(r)$

$$
\Delta d_{\mathrm{eff}} \approx \frac{\Delta d}{\sqrt{1+\frac{I_{\text {peak }}}{I_{\mathrm{s}}} \frac{e \Delta d^{2}}{2 w_{0}^{2}}}} .
$$

Taking into account that the diameter of the diffraction-limited excitation focal spot ( $\Delta d \approx 230 \mathrm{~nm}$ for $\lambda_{\mathrm{exc}}=635 \mathrm{~nm}$ ) is comparable to the parameter $w_{0}$ of the red-shifted Laguerre-Gaussian STED focus 
a

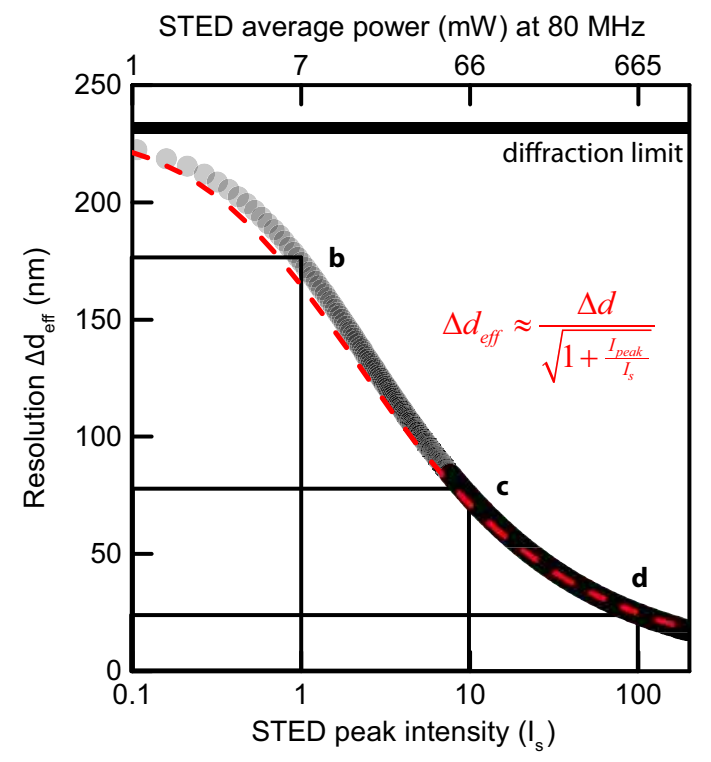

b

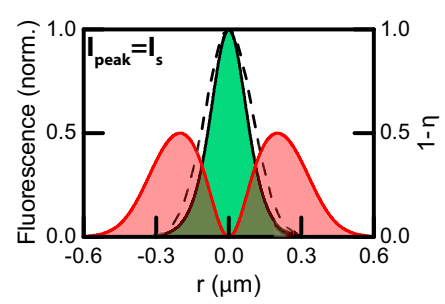

c
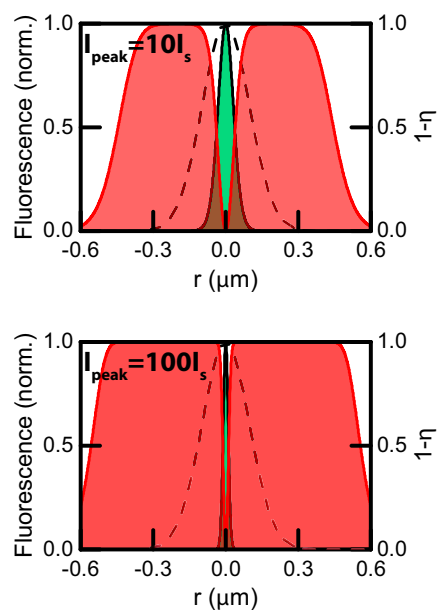

Figure 2.3: Resolution scaling in STED microscopy. (a) Resolution scaling with STED peak intensity for the STED pulse duration $\tau_{\mathrm{STED}}=200 \mathrm{ps}$ and the repetition rate $f=80 \mathrm{MHz}$. Gray dots represent resolution calculated numerically from Equation 2.9 (FWHM); dashed red line is a fit of the shown expression to the numerical data. Diffraction limited resolution $\Delta d \approx 230 \mathrm{~nm}$, saturation intensity $I_{\mathrm{s}} \approx 90 \mathrm{MW} / \mathrm{cm}^{2}$. (b-d) Diffraction-limited PSF (dashed black line), residual fluorescence (green) and fluorescence suppression factor (1- $\eta$ ) (red) for the peak intensity $I_{\text {peak }}=I_{\mathrm{s}}$ in (b), $I_{\text {peak }}=10 I_{\mathrm{s}}$ in $(\mathrm{c})$ and $I_{\text {peak }}=100 I_{\mathrm{s}}$ in $(\mathrm{d})$.

$\left(w_{0} \approx 285 \mathrm{~nm}\right.$ for $\left.\lambda_{\mathrm{STED}}=750 \mathrm{~nm}\right)$, the term $\frac{e \Delta d^{2}}{2 w_{0}^{2}} \approx 1$ and Equation 2.11 simplifies to the well-known inverse square-root law of resolution scaling in STED nanoscopy ${ }^{82}$

$$
\Delta d_{\mathrm{eff}} \approx \frac{\Delta d}{\sqrt{1+\frac{I_{\text {peak }}}{I_{s}}}}
$$

The resolution scales inversely with the square root of the ratio between STED intensity and parameter $I_{s}$. For a STED wavelength of $\lambda_{\mathrm{STED}}=750 \mathrm{~nm}$, a STED pulse duration of $\tau_{\mathrm{STED}}=200 \mathrm{ps}$ and a crosssection for stimulated emission at the STED wavelength $\sigma_{\mathrm{STED}} \approx 1 \times 10^{-17} \mathrm{~cm}^{2}, I_{S}$ equals (Equation 2.8 )

$$
I_{\mathrm{S}} \approx \frac{0.69 \cdot 1 \times 10^{-34} \mathrm{~J} \mathrm{~s} \cdot 2.5 \times 10^{15} \mathrm{~s}^{-1}}{1 \times 10^{-17} \mathrm{~cm}^{2} \cdot 200 \times 10^{-12} \mathrm{~s}} \approx 90 \mathrm{MW} / \mathrm{cm}^{2}
$$

The resolution scaling with STED intensity is shown in Fig. 2.3. The STED peak intensities required for a substantial resolution gain $\left(\geq 10 I_{S}\right)$ are higher than the intensities applied in conventional onephoton excitation fluorescence microscopy $\left(\sim 100 \mathrm{~kW} / \mathrm{cm}^{2}\right)$, but lower than the excitation intensities in two-photon microscopy $\left(\sim 100 \mathrm{GW} / \mathrm{cm}^{2}\right)$. Conceptually, even high STED intensities should be harmless for fluorophores as photons are applied to de-excite molecules in the reactive excited state to the non-reactive ground state. Unfortunately, at high STED intensity levels (resolutions), the absorption events of STED-light photons by excited fluorophores (or neighbor molecules) cannot be neglected. The resulting population of chemically reactive molecular excited states (singlet and triplet) 
results in photobleaching observable as a permanent loss of fluorescence signal during scanning. In addition to photobleaching, such high irradiation can cause significant phototoxicity in living systems.

\subsubsection{Photobleaching of molecular probes}

Photoinduced degradation of fluorescent markers is a practical limitation for all super-resolution microscopy techniques. Both single-molecule localization and coordinate-targeted methods suffer from the fact that each fluorophore is characterized by the finite number of excitation-emission cycles, which limits the detected fluorescence signal. In traditional fluorescence microscopy and coordinatestochastic nanoscopy, nearly all of the excitation events result in emission of fluorescence photons. This means that the majority of the optical transitions are contributing to the image formation. In STED microscopy, a large portion of the optical transitions are applied to suppress the fluorescence emission by stimulated emission and thus are not registered as signal. The conceptually harmless STED photons in practice influence the markers' survival time by considerable absorption events within a complex molecular energy structure, which results in a higher chemical reactivity of the excited organic compounds. Therefore, photostability of the fluorophore under STED-wavelength illumination plays an essential role for successful imaging.

Photobleaching refers to any chemical process mediated by the excited state of a fluorophore which results in a permanent loss of the marker's ability to emit fluorescence. Only excited molecules are at the risk of photobleaching as 'good fluorophores' are assumed to be chemically stable in the normally occupied ground state $S_{0}$. The magnitude of photobleaching depends on the illumination, on the specific dye and its neighboring molecules, which facilitate certain chemical reactions. Promotion of an electron to one of the excited states by absorption of a photon is followed by rearrangements of the molecular electronic structure. Thus, the excited molecule has photochemical properties different than the same molecule in the ground state, and often is considered as a 'different species' ${ }^{83}$. Upon excitation, the excess of energy is relatively quickly released by emission of a photon (called fluorescence or phosphorescence), heat (e.g., vibrational relaxation), chemical energy (e.g., dissociation) or energy transfer to another molecule (e.g., FRET). In general, molecules in higher excited states are more vulnerable to photobleaching as the electronic bond is weaker. Therefore, the primary step of photobleaching depends on the excitation of molecules by absorption of light at a certain photon energy. Photobleaching initially has been studied in fluorescent dye solutions and solid matrices. It was a very active research area as dyes had many practical applications including their role as photosensitizers in photography or lasing mediums in dye lasers. Many of these early experiments gave a good foundation for our understanding of photobleaching mechanisms and further investigations of light-induced damage in optical microscopy conditions. The early experiments showed that bleaching is proportional to the absorption of light by the system. It was noticed that the lifetime of an excited state is of crucial importance for the further photodamage pathway as the chemical reaction has to occur within that time. The first photobleaching models considered only the damage mediated by long-lived states in the singlet and triplet system: the first excited singlet state and the first triplet state $\left(S_{1}, T_{1}\right)$. For short-lived singlet states, only very fast chemical processes can occur (e.g., dissociation). 
The long-lived states have a high chance to undergo slow chemical reactions (e.g., redox reactions with neighboring molecules). The first experiments revealed the complexity of photobleaching processes, which strongly depend on the class of the dyes in use, solvent polarity, temperature, $\mathrm{pH}$ and the energy of illuminating photons ${ }^{83,84}$.

Photobleaching measurements under fluorescence microscopy conditions for free and chemically bound fluorescent markers confirmed the involvement of the first excited states $\left(S_{1}, T_{1}\right)$ in photodamage at low irradiation levels ${ }^{19,20,22,85,86}$. One pathway of photobleaching was identified as photooxidation of excited fluorophores occupying $T_{1}$. The electron transfer reaction to molecular oxygen results in singlet oxygen and a non-fluorescent radical species of the fluorophore. Singlet oxygen and radicals can further react with fluorophores, causing photobleaching of markers and phototoxicity in living cells. This pathway was proven by the strong impact of oxygen presence in the photobleaching of several dyes $19,20,85,87$. Samples prepared under argon-saturated conditions were typically characterized by minimized (but nonzero) photodamage ${ }^{19,20}$. Direct photobleaching reactions from the first excited states $\left(S_{1}, T_{1}\right)$ result in bleaching proportional to the population of $S_{1}$, called low-order photobleaching. There, photodamage effectively scales with light intensity, as does the fluorescence signal. Therefore, in one-photon excitation (1PE) microscopy, low-order photobleaching $B$ is proportional to the excitation intensity $\left(B \propto I^{b}, b=1\right)$ whereas in two-photon excitation (2PE) microscopy the same mechanism of damage leads to a quadratic dependence $(b=2)$. Low-order photobleaching has been observed for fluorescent dyes and some fluorescent proteins at low irradiances in 1PE microscopy ${ }^{21,88,89}$. More intense photon fluxes, however, result in a higher order $b$ of photobleaching, and a simple three-level system $\left(S_{0}, S_{1}, T_{1}\right)$ is by no means sufficient to explain the experimental observations ${ }^{85,89,90}$. For 1PE microscopy, a photobleaching scaling $B \propto I^{b}$ with $b>1$ has been reported for fluorescent dyes and many fluorescent proteins ${ }^{22,85,89}$. Even higher photobleaching orders $b$ have been reported in $2 \mathrm{PE}$ fluorescence microscopy with $b=3-5$ for organic fluorophores ${ }^{21,22}$ and green fluorescent protein (GFP) ${ }^{88,90}$. Such scalings indicate the involvement of higher excited electronic states in the markers' photodamage. This bleaching regime is called high-order or accelerated photobleaching. High-order bleaching is often dominant in multiphoton fluorescence microscopy. One of the mechanisms of photodamage is a dissociation of the excited electron (in $S_{n}$ or $T_{n}$ ) by a solvent cation. This pathway is strongly influenced by the polarity of the solvent in use ${ }^{22}$.

In STED microscopy, the intense photon flux is applied to suppress the fluorescence ability of molecular probes by stimulated emission $\left(S_{1} \rightarrow S_{0}\right)$. Thus, in the ideal case STED photons protect the fluorophores from following the photodamage pathways starting at $S_{1}$. In practice, however, depending on the photophysical properties of the molecules and the environment, intense STED light can also cause excitation of markers. Of particular importance seems to be the excited state absorption(ESA) the spectrum of which is often unknown. ESA refers to any absorption events starting from the excited states of the fluorophore. STED-light photons can directly transfer the excited molecule in a singlet or triplet state to any energetically matching higher-excited electronic state with the same multiplicity (e.g., $S_{1} \rightarrow S_{n}, T_{1} \rightarrow T_{n}$ ). The excitation can occur in one- or multi-photon absorption processes. The population of higher excited states with respective photobleaching probabilities results in a STED 
intensity-dependent photobleaching. Knowledge of the photobleaching order $b$ in STED microscopy $\left(B \propto I_{\text {STED }}^{b}\right)$ would greatly benefit the experimental strategies, by providing information on the optimal de-excitation pulse duration which minimizes STED-light-induced damage.

The involvement of triplet-mediated ESA $\left(T_{1} \rightarrow T_{n}\right)$ in photobleaching under STED microscopy conditions has been proven for several dyes by the success of strategies allowing triplet state relaxation, as T-Rex or fast scanners ${ }^{29,30,91,92}$. Dramatic differences have been observed in the STED performance for dyes with different ESA spectra in the singlet system $\left(S_{1} \rightarrow S_{n}\right)^{31}$. However, none of these experiments revealed to which extent the photobleaching depends on the STED intensity. One of the reasons is that simple measurements of photobleaching as a function of STED intensity by varying the time-averaged STED power usually lead to a sublinear bleaching dependence $b<1$. Such a scaling is the result of a drastic decrease of $S_{1}$ population with increased number of STED photons (and thus decrease of photobleaching magnitude). Therefore, measurements of photobleaching where the STED average power is varied hide the involvement of higher excited molecular states $S_{n}, T_{n}$ in STED-light-induced damage. Investigations with the aim to extract the photobleaching order $b$ under STED microscopy conditions necessitate a constant de-excitation energy, such that, while changing the STED intensity, the population of the excited state $S_{1}$ is roughly constant.

To describe the aforementioned light-induced damage observed in STED microscopy experiments, a five-level system (including $S_{n}, T_{n}$ with $n>1$ ) has to be considered (Fig. 2.4a). The schematic model of the relevant electronic energy states is based on previous work on photobleaching in fluorescence microscopy ${ }^{85}$. Each of the excited states $\left(S_{1}, S_{n}, T_{1}, T_{n}\right)$ is characterized by its own photobleaching rate represented schematically by a pink arrow. Upon excitation $\left(S_{0} \rightarrow S_{1}\right)$, the excited electron can release energy by emission of a photon (by fluorescence $S_{1} \rightsquigarrow S_{0}$ or stimulated emission $S_{1} \rightarrow S_{0}$ ), be promoted to a higher excited singlet state by absorption of a STED-light photon $\left(S_{1} \rightarrow S_{n}\right.$, ESA) or with a certain probability undergo intersystem crossing $\left(S_{1} \rightsquigarrow T_{1}\right.$, ISC) and enter the first excited triplet state $T_{1}$. The probability of ISC is typically low (ISC $<0.01$ ) as direct optical transitions between states with different multiplicity are forbidden. ISC requires spin flipping of the excited electron. Despite its low probability, $T_{1}$ population can be easily built up in common microscopy setups due to the large number of excitation cycles undergone. Demands for high fluorescence photon fluxes require high repetitionrate lasers, which does not allow for relaxation of molecules trapped in the long-lived first excited triplet state (with lifetime from micro- to milliseconds) between subsequent pulses. After multiple excitation events, the $T_{1}$ population can be substantial. The STED-light photons can further excite molecules in the first excited triplet state to a higher excited triplet state $\left(T_{1} \rightarrow T_{n}\right.$, ESA). Moreover, STED-light photons can cause excitation of 'hot' ground-state molecules by one-photon absorption ( $S_{0} \rightarrow S_{1}$ ) or excitation of ground-state molecules by multiphoton absorption to a higher excited singlet state ( $S_{0} \rightarrow \rightarrow S_{n}$ ). Higher excited singlet states $S_{n}$ tend to relax relatively quickly (within picoseconds) to $S_{1}$ by internal conversion. Therefore, mechanisms of damage mediated by the singlet system can be subsumed to the first excited singlet state, with an effective photobleaching probability dependent on the STED light intensity. The triplet-related photodamage occurs on a totally different time scale. For example, the second excited triplet state $T_{2}$ can be characterized by a lifetime of the order $100 \mathrm{~ns}$. 

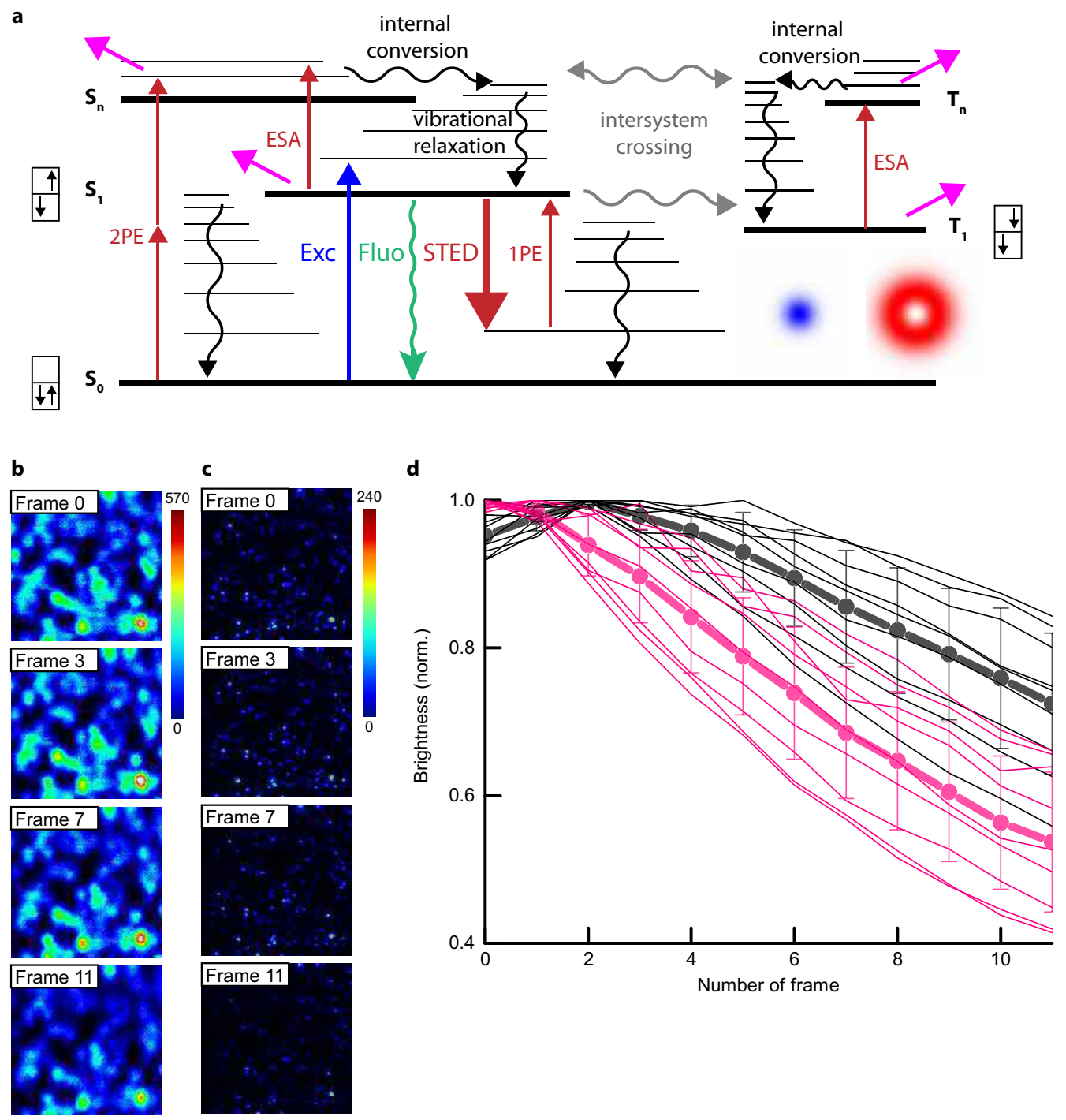

Figure 2.4: Photobleaching in STED microscopy. (a) Simplified model of states possibly involved in photobleaching, including a higer excited singlet state $S_{n}$, the first excited triplet state $T_{1}$ and higher excited triplet state $T_{n}$. 1PE: one-photon excitation by STED light, 2PE: two-photon excitation by STED light, ESA: excited-state absorption by STED light. (b-c) Consecutive images of crimson fluorescent beads dispersed on a cover glass under confocal (b) and STED (c) conditions. Exc: $\lambda_{\text {exc }}=635 \mathrm{~nm}, \tau_{\mathrm{exc}}=500 \mathrm{fs}, P_{\mathrm{exc}}=20 \mu \mathrm{W}$, STED: $\lambda_{\mathrm{STED}}=750 \mathrm{~nm}, \tau_{\mathrm{STED}}=500 \mathrm{ps}, P_{\mathrm{STED}}=150 \mathrm{~mW}$, repetition rate $80 \mathrm{MHz}$, FOV: $5 \times 5 \mu^{2}\left(90 \times 90 \mathrm{px}^{2}\right)$, pixel dwell time: $0.5 \mathrm{~ms}$. (d) Changes in brightness shown in (b-c) for confocal (black) and STED mode (pink) of different regions of the crimson bead sample. The bold lines represent the mean value with standard deviation as the error of measurement.

The situation is even more complex, as the excited molecules in the triplet system can undergo reverse ISC and return into the singlet system $\left(T_{n} \rightsquigarrow S_{n}\right)$. Fluorescence emission from molecules which passed through the triplet system is called delayed fluorescence. Delayed fluorescence has the same spectrum as the singlet spontaneous emission, but a much longer emission time ( $\sim 100 \mathrm{~ns})$.

Additionally to the complex dynamics of the molecular electronic states, the STED beam intensity distribution in the focal plane causes a spatially varying photobleaching probability. The effects of photodamage measured as changes in fluorescence signal (brightness) in a STED microscope 
are integrated in the confocalized bucket detector. Such measurements often lead to an observable biexponential signal decay even for the simplest case of a single photobleaching process from the excited state $S_{1}$. Therefore, the photodamage observed in STED microscopy is often difficult to interpret. As an example, the brightness of crimson fluorescent beads over sequential imaging frames is shown in Fig. 2.4b-d for the confocal and STED imaging modes. The fluorescent beads were dispersed on the cover glass. The Gaussian excitation and doughnut-shaped STED beams were nearly diffraction-limited. Confocal measurements were taken with the excitation beam operating at a wavelength $\lambda_{\mathrm{exc}}=635 \mathrm{~nm}$, with a pulse duration of $\tau_{\mathrm{exc}}=500 \mathrm{fs}$ and a time-averaged power of $P_{\text {exc }}=20 \mu \mathrm{W}$ at $80 \mathrm{MHz}$ repetition rate. Figure $2.4 \mathrm{~b}$ shows one data set of such measurements, where the same FOV $\left(5 \times 5 \mu \mathrm{m}^{2}\right)$ was imaged in twelve sequential frames. The total brightness was calculated as the sum of the signal from individual pixels. The results from several measurement series are plotted in Fig. 2.4d (black lines). Each line represents the same measurement series as presented in Fig. 2.4b for a fresh area of the fluorescent bead sample. The bold line represents the mean value of the respective data set, with standard deviation as the error of measurement. Clearly, the detected signal (brightness) changes over the time of light exposure. However, even in the simple confocal case the results are surprising. The total brightness initially increases, reaching its maximal value roughly for Frame 2. Increases of signal upon light exposure have been observed for densely packed fluorophores ${ }^{93,94}$. Such a behavior is explained by self-quenching of excited molecules via energy transfer processes between adjacent fluorescent molecules. The quenching can be minimized by increasing the average distance between the fluorophores. The same measurements taken in the STED mode are shown in Fig. 2.4c,d. The excitation parameters were the same as for the confocal measurements. The STED parameters were as follows: $\lambda_{\mathrm{STED}}=750 \mathrm{~nm}, \tau_{\mathrm{STED}}=500 \mathrm{ps}, P_{\mathrm{STED}}=150 \mathrm{~mW}$ at $80 \mathrm{MHz}$, corresponding to $\sim 4$ times resolution improvement. Exposure to the STED-light photons on average accelerated the loss of signal (Fig. 2.4d, pink bold line). However, significant differences among the individual series (reflected by the error of measurement) did not allow for more quantitative conclusions. To obtain a quantitative comparison, the analysis of photobleaching requires a simpler model sample.

It is important to note that photobleaching in STED microscopy can also be minimized by strategies which reduce unnecessary exposure of molecular probes to the intense STED light. In these methods, additional knowledge about the mechanism of photobleaching is not necessarily required. The light exposure can be shortened in time by an intelligent scanning strategy which adapts the pixel dwell time in response to the registered fluorescence signal at a given time $\left(\mathrm{RESCue}^{95}\right)$. Alternatively, the exposure can be diminished by taking into account the spatial distribution of markers in the sample. To do so, a preliminary image has to be recorded containing a rough estimation of the molecular positions (e.g., a confocal image). Then, the exposure to STED light can be limited for instance by restriction of the scanning to just subdiffractional regions around the expected molecular positions (MINFIELD ${ }^{96}$ ). Thus, the markers experience rather low STED-light intensities around the coordinate targeted by the doughnut 'zero'. Another approach is based on the dynamic pixel-to-pixel adaptation of the STEDlight intensity (DyMIN ${ }^{97}$ ). The STED intensity is kept relatively high only when it is beneficial, that is, nearby the estimated molecular positions. Furthermore, photobleaching in STED microscopy can 
be reduced by utilizing multiple states of a fluorophore in an image acquisition scheme. For example, reversibly photoswitchable fluorescent proteins can be reversibly transformed to a non-fluorescent (non-absorbing) variant upon absorption of a certain photon energy. By applying an additional doughnut-shaped beam, operating at the wavelength matching this transition, fluorescent proteins can be kept in a non-fluorescent (non-absorbing) state at a high intensity of the STED doughnut crest (protected STED ${ }^{98}$ ). Thus, protein variants which are not optically active at the STED wavelength are secured from the STED-light-induced damage.

\subsection{GSD nanoscopy with emitters resistant to photobleaching}

\subsubsection{Quantum emitters alternative to molecular probes}

Organic fluorophores are one of the most popular contrast agents in optical nanoscopy. Certain advantages, such as their small size (Fig. 2.5a), variety of colors and well-established labeling procedures make them convenient probes for targeting the structures of interest. Nonetheless, organic dyes have several drawbacks such as limited brightness, wide emission spectra or only moderate photostability, which limit their performance in imaging. Moreover, not all nanoscopy techniques are easily applicable with common fluorescent molecules. While coordinate-targeted strategies like STED ${ }^{18}$ microscopy are relatively straightforward, the coordinate-stochastic methods (e.g., STORM ${ }^{6}$ ) require an organic dye or even a pair of dyes interacting with a special host buffer (solution). The role of the medium is to promote a long-lived 'off' state of the molecules and thus make it possible to resolve just a sparse subset of the population in an 'on' state during each frame exposure of a camera. This requirement makes single-molecule localization microscopy with organic probes often unsuitable for live-cell experiments, as the utilized buffer is also toxic.

The other popular class of labels, photoswitchable fluorescent proteins, is highly compatible with live-cell imaging and widely applied in coordinate-targeted and -stochastic optical nanoscopy (e.g., RESOLFT $^{99}$, PALM $^{7}$ ). Fluorescent proteins have a barrel-like structure, bigger in size than the organic compounds (Fig. 2.5b). The complex arrangement of amino acids protects the chromophore (shown in red) from the environment ${ }^{100}$. The non-fluorescent form of the photoswitchable protein ('off') can be transformed into the fluorescent variant ('on') upon absorption of a certain photon energy, followed by the rearrangement of the protein structure. Depending on the response to light, this process can be reversible (e.g., cis-trans isomerization) or irreversible (e.g., covalent changes in chromophore structure $)^{101}$. The clear advantage of photoconvertible fluorescent proteins over the organic dyes is the relatively low photon flux necessary for 'on' $\leftrightarrow$ 'off' optical switching. This property makes them attractive in biological research, as possible phototoxic effects related to light exposure of the living system are diminished. The trade-off is a rather slow switching kinetics. Furthermore, fluorescent proteins typically feature lower brightness than organic dyes, which compromises the contrast. These drawbacks are especially severe in point-scanning optical nanoscopy with reversibly photoswitchable fluorescent proteins, resulting in relatively slow image acquisition.

Alternative probes for optical nanoscopy are nanocrystals, including color centers in nanodia- 


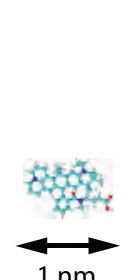

b

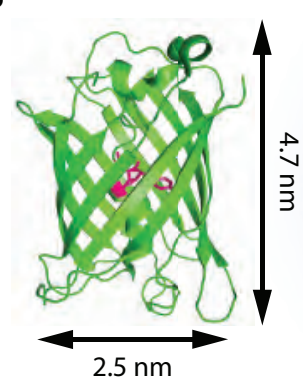

c

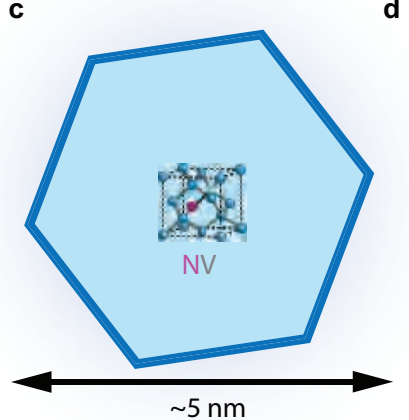

d

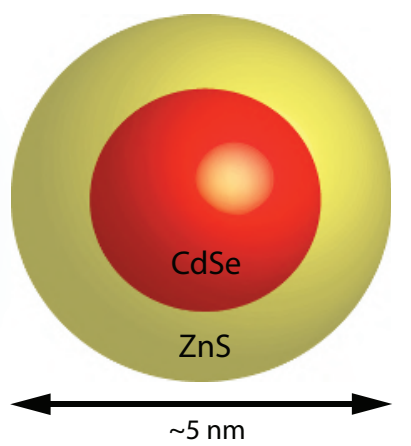

Figure 2.5: Emitters imaged by super-resolution microscopy techniques. (a) Organic dye ATTO647N. (b) Green fluorescent protein (GFP) with a barrel-like structure of amino acids surrounding the chromophore (red). (c) Schematic drawing of point-like NV impurity in a nanodiamond crystal lattice. (d) Schematic drawing of a (CdSe)ZnS core-shell quantum dot.

monds ${ }^{9,15}$, excitonic quantum dots ${ }^{10-12}$ and upconversion nanoparticles ${ }^{13,14}$. The major advantage over the aforementioned fluorophores is a higher brightness and robust photon emission. The benefits come in line with challenges related to their rather substantial size $(\sim 10 \mathrm{~nm})$, complicated surface chemistry necessary to accomplish bio-targeting, limited cell permeability and often increased toxicity. Because of that, the potential of nanocrystalline particles in live imaging is still controversial.

The most successful emitters imaged by coordinate-targeted super-resolution microscopy are the atomic-scale defects in the diamond crystal lattice called NV centers. One or more defects can be implanted in the bulk material or in form of nanodiamond particles. Nanodiamonds can be synthesized with a size down to a few nanometers, as presented schematically in Fig. 2.5c. The pure diamond crystal is a transparent material with a broad transmission range, due to a large bandgap of $\sim 5.5 \mathrm{eV}$. The variety of diamond colors result from many different impurities in the crystal structure (color centers). The NV center is the most popular impurity, constituting a nitrogen atom (in the carbon position) next to a charged vacancy site. The electrons of this defect form a triplet system which can be excited by photons in the visible spectral range. Upon excitation, the NV center emits bright, red fluorescence that is easily detectable even from individual impurities at room temperature. As the emission results from relaxation of a higher excited triplet state, the fluorescence lifetime is relatively long $\left(\tau_{\mathrm{fl}}=11 \mathrm{~ns}\right)$. Typically, neither transient emission intermittency (blinking) nor permanent bleaching is observed, as the color centers are protected from the environment by a very rigid diamond crystal structure. However, for small nanodiamonds ( $\sim 5 \mathrm{~nm}$ in size) emission intermittency can occur ${ }^{102}$. Moreover, the electron spins of NV centers can be easily polarized by external perturbations, such as magnetic fields or microwaves, and read out by changes of the fluorescence signal. Therefore, nanodiamonds with NV centers are particularly interesting as biolabels or nanosensors of local electric and magnetic fields with single-electron field sensitivity. 
The very rapid growth of nanotechnology brings novel particles with optical properties that can be utilized to accomplish super-resolution imaging. One type of particles are photoluminescent quantum dots (QDs). Photoluminescent QDs are semiconducting nanoparticles which, as a result of small dimensions, restrict the free motion of excited electrons normally occurring in the bulk material. The restriction of motion in three dimensions results in discrete electronic energy levels. The relative separation between the different electronic states depends on the geometry of the nanoparticle. Thus, QDs' energy structure is similar to that naturally occurring in atoms and molecules. This cluster of atoms effectively acts as one giant particle and is often referred to as an 'artificial atom'. Photoluminescence in QDs can occur upon promotion of a valence-band electron to an empty excited state in the conduction band by, for instance, absorption of a photon. The excited electron leaves the empty energy state in the valence band called an electron hole. The hole has a net positive charge and is attracted to the excited electron by the Coulomb interaction. The electron-hole pair creates a quasi-particle called an exciton. Exciton energy can be released by recombination of the electron with the hole, accompanied by emission of a photon. The properties of electron-hole pair recombination, such as lifetime or spectra, can vary for a given material composition with the size of the nanoparticle. This effect is called quantum confinement and occurs when the size of the nanoparticle is on the order of the physical separation between electron and hole in bulk material given by the exciton Bohr radius. QDs have a relatively narrow emission band $(<50 \mathrm{~nm})$, which is advantageous in multiplexing, as different labels can be separated with low cross-talk between individual color channels. The significant difference between QDs and common fluorophores lies in very broad absorption spectra of the former. QDs at room temperature can absorb nearly any photon with an energy higher than the bandgap. The absorption cross-section increases approximately linearly with the excitation photon energy. This feature is advantageous in conventional optical microscopy, where multiple labels can be excited with just a single UV laser, but it becomes problematic with strategies like STED ${ }^{12}$. STED-light photons applied with the intention to de-excite often cause increased excitation of the emitters by multiphoton absorption. Another problem is the QD emission intermittency (blinking). Widely accepted blinking mechanisms are associated with either light-induced charging of the QD core (opening nonradiative Auger-like recombination channels) or electron-accepting surface sites which could intercept the electrons ${ }^{103,104}$. The second type of blinking is related to tunneling of 'hot' electrons outside the nanocrystal core and can be suppressed by a wider bandgap semiconductor shell of a proper thickness. Despite some attempts ${ }^{53}$, the relatively short lifetime of QD 'off' states makes it difficult to employ this property in coordinate-stochastic super-resolution microscopy.

The last emitters mentioned here are upconversion nanoparticles. Upconversion is anti-Stokes emission upon excitation by absorption of several low-energy photons. This behavior is characteristic of nanocrystals doped with ions such as thulium or praseodymium. The advantages of using such emitters in microscopy lie in their high photostability and ability of background-free fluorescence imaging, resulting from the relatively long excitation wavelength. Upconversion nanocrystals can be synthesized with a size of down to $\sim 10 \mathrm{~nm}$. Their optical properties, such as a long lifetime ${ }^{13}$ or a photo-avalanche-like effect between adjacent ions within a single nanoparticle ${ }^{14}$, make it possible to 
acquire subdiffractional images at relatively low light intensities.

\subsubsection{GaP-GaInP heterostructured nanowires}

The term 'nanowires' (NWs) refers to high-aspect-ratio nanostructures with a diameter of $<100 \mathrm{~nm}$ and length from several to hundreds of micrometers. The properties of the material at the nanoscale differ from the properties of the same material in bulk. Thus, NWs are often characterized by enhanced optical and elastomechanical properties, ballistic electrical conductivity or exceptional sensitivity to environmental conditions related to their substantial surface-to-volume ratio ${ }^{105}$. Of particular interest are semiconductor NWs, as their electro-optical properties can be tuned by changing the material composition. The most common NWs are built from III-V semiconductor compounds. Their absorption and emission spectra can cover nearly the entire visible and infrared spectral range. Additionally to the broad choice of materials, NWs can be synthesized as heterostructures by substituting two or more alloys within a single wire in axial or radial (core-shell) direction.

Due to their aforementioned unique properties, these nanoneedles have gained considerable attention in many areas of science and technology, including biological research. In biology, NWs have been used for cell guidance, as injection devices and as sensors of local electric fields or local forces at the cell scale (see reviews, ref. ${ }^{106,107}$ ). The mechanisms of interaction between the inorganic nanomaterials and a living system, however, are not yet fully understood ${ }^{108}$. NWs, due to excellent controllability of their synthetic growth, are a promising model sample in nanotoxicity studies ${ }^{109}$. All of these applications necessitate visualization methods suitable for biological conditions.

In this thesis, gallium phosphide-gallium indium phosphide (GaP-GaInP) axial heterostructured NWs are studied. The widely used semiconductor GaP has an indirect bandgap, which means that, upon electronic excitation, the optical relaxation requires an involvement of the crystal lattice vibrations called phonons. This process occurs with a very low probability and therefore GaP photoluminescence is typically not observed ${ }^{44}$. On the other hand, the GaInP alloy has a direct bandgap for InP concentration $>0.3$ Ref. 110 . The electron-hole recombination over the bandgap results in a bright and robust photoluminescence signal at room temperature. The bandgap and thus the photoluminescence spectrum can be tuned from the visible to the near infrared region $(560-920 \mathrm{~nm})$ by changing the molar fraction of indium.

Inherently luminescent $\mathrm{GaP}-\mathrm{GaInP}$ heterostructures have been grown to overcome the challenges of NW visualization encountered in previous studies ${ }^{44}$. For example, NWs imaged by scattered photons featured a low contrast. In biological specimens, they could be easily confused with other scattering components. Better contrast has been achieved by targeting the NW's surface with fluorescent probes ${ }^{39,41}$. The use of organic molecular probes, however, limits time-lapse studies due to photobleaching and also influences the surface chemistry of the wires. The NWs' morphology and surface chemistry are expected to play a crucial role in their nanotoxicology. Combination of nonluminescent $\mathrm{GaP}$ and luminescent $\mathrm{GaInP}$ segments within a single wire creates a distinctive barcode pattern, which can be employed for visualization and identification of nanowires featuring different chemical and morphological properties in cells and tissues ${ }^{44}$. 
In general, there exist two approaches to produce nanowires: the top-down and the bottom-up approach. The top-down approach reduces the size of an initially big object to create a nanometerscale structure. It is used relatively rarely, for example, to produce an optical NW by stretching the optical fiber ${ }^{111}$. The bottom-up approach is more popular, a good example being metalorganic vapor phase epitaxy (MOVPE). MOVPE relies on chemical vapor deposition on a base substrate. All NWs investigated in this thesis were grown at the Center for Nanoscience of Lund University using MOVPE. MOVPE provides a high degree of control, flexibility and reproducibility of the synthesized nanostructures. The 1D NW growth was assisted with catalyst gold particles placed on a GaP substrate. The diameters of the deposited Au-particles roughly correspond to the final NW diameters. Next, the substrate was placed in the MOVPE reactor chamber and precursor gases were added at controllable flow rates. At temperatures of $>400{ }^{\circ} \mathrm{C}$, eutectic droplets of the metal particles and the reactant gases were formed, followed by the deposition of the semiconductor material underneath the gold particle. The wires' length depends on the reaction duration. By changing the composition of the precursor gases, axial heterostructures with designed segment compositions and lengths were formed.

All NWs studied here were detached from the GaP substrate by sonication and deposited on the cover glass. Examples of conventional confocal images of GaP NWs containing a single photoluminescent GaInP segment are presented in Fig. 2.6a,d. The excitation wavelength equals $\sim 700 \mathrm{~nm}$. The confocal images show the scattered excitation light (gray) overlapping photoluminescence signal from GaInP segments extracted by a longpass filter (red). The photoluminescence signal provides significantly higher contrast than the scattered light. In the case of relatively large GaInP segments (such as in Fig. 2.6a), neutral density filters had to be placed in the detection path to avoid saturation of the detector. When the size of the luminescent segment is decreased, the signal scales roughly proportionally to the GaInP volume. As the large GaInP segments are very bright (Fig. 2.6a), we were able to measure the photoluminescence spectrum from the single GaInP segment (Fig. 2.6b). GaInP photoluminescence had a central wavelength of $760 \mathrm{~nm}$ and a bandwidth $<70 \mathrm{~nm}$. Due to poor time resolution of the detector, the photoluminescence lifetime (Fig. 2.6c) was only roughly estimated to $<100 \mathrm{ps}$. The detector time resolution was measured as the instrument response function (IRF) to an ultrashort laser pulse ( $\tau \approx 500 \mathrm{fs})$.

Even short GaInP segments featured robust emission. An image of $20 \times 20 \mu \mathrm{m}^{2}\left(512 \times 512 \mathrm{px}^{2}\right)$ in size with a pixel dwell time of $0.5 \mathrm{~ms}$ can be collected repeatedly over hours with a relatively high excitation power $P_{\text {exc }}=1.5 \mathrm{~mW}$ at $80 \mathrm{MHz}$ repetition rate and an excitation pulse duration of $\tau_{\text {exc }}=5 \mathrm{ps}$ (Fig. 2.6d). The brightness of GaInP segments in sequential frames is plotted in Fig. 2.6e,f. Despite the differences of parameters between the brightness measurements for NWs and those of molecular probes presented previously (compare Fig. 2.6e,f to Fig. 2.4d black lines), it is apparent that the internal GaInP photoluminescence offers a superior photostability over fluorescent beads.

GaP-GaInP NWs with diameter smaller than $40 \mathrm{~nm}$ exhibit emission intermittencies. The signal fluctuation decreases with increased excitation power (Fig. 2.6g,h). For artificial structures, emission intermittency is expected to occur when their size approaches the exciton Bohr radius of the material. For GaInP, the exciton Bohr radius equals $\sim 4-10 \mathrm{~nm}$. 


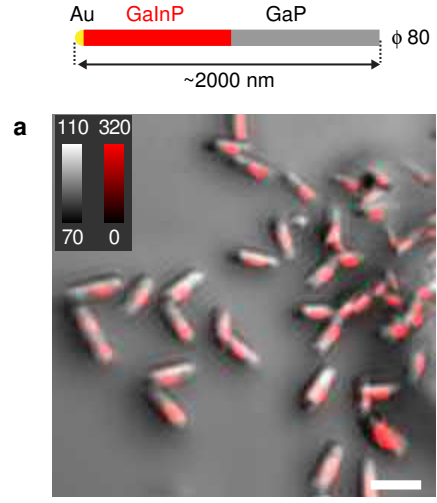

b
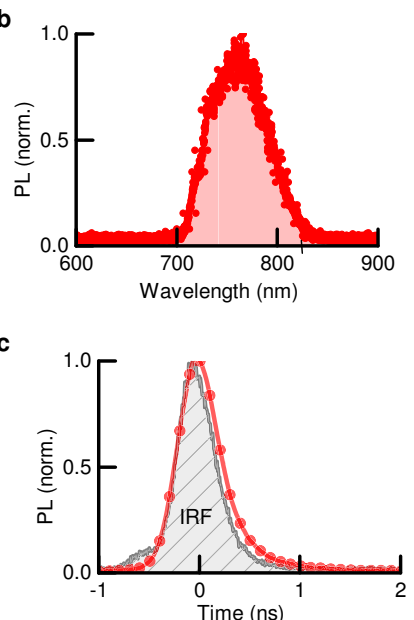

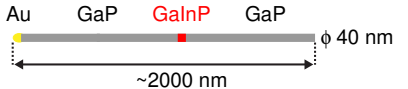

d
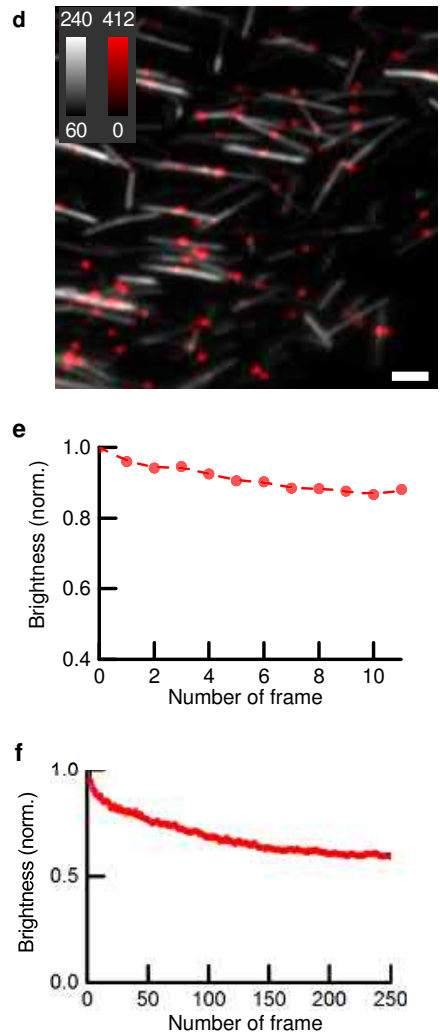
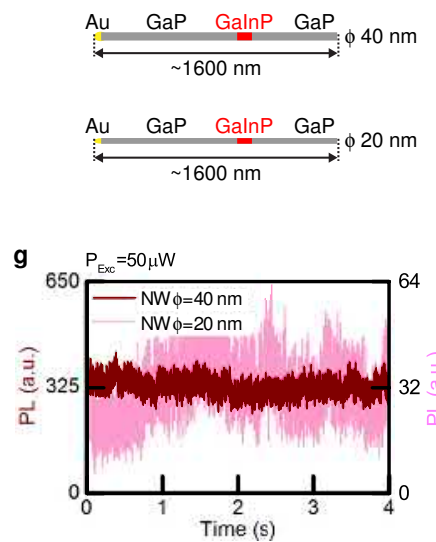

$\stackrel{\mathrm{Au} \quad \mathrm{GaP} \quad \text { GalnP GaP }}{\rightleftarrows} \mathrm{i} 20 \mathrm{~nm}$

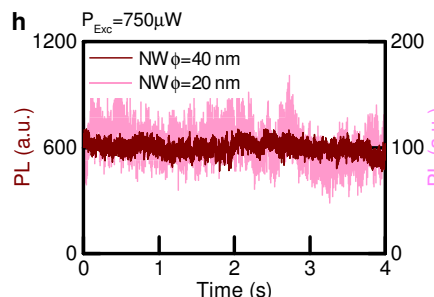

Figure 2.6: Heterostructured GaP-GaInP nanowires with a single GaInP photoluminescent segment. (ac) GaP nanowires with a relatively long GaInP segment. Conventional optical microscopy image, the gray scale represents the scattering signal and the red scale represents photoluminescence (a). GaInP photoluminescence (PL) spectrum (b). GaInP photoluminescence lifetime measurement (red) with instrument response function (IRF, gray, $\tau_{\text {jitter }} \approx 430 \mathrm{ps}$ ). (d-f) GaP nanowires with a short GaInP segment. Example of a scattering (gray) and photoluminescence (red) image (d). Normalized brightness of nanowires in consecutive frames showing the excellent photostability (e,f). Exc: $\lambda_{\mathrm{exc}}=700 \mathrm{~nm}, \tau_{\mathrm{exc}}=5 \mathrm{ps}, P_{\mathrm{exc}}=1.5 \mathrm{~mW}$, repetition rate: $80 \mathrm{MHz}$, FOV: $20 \times 20 \mu \mathrm{m}^{2}\left(512 \times 512 \mathrm{px}^{2}\right)$, dwell time: $0.5 \mathrm{~ms}$. All scale bars: $2 \mu \mathrm{m}$. (g,h) Emission intermittencies detected for nanowires with different diameters for excitation powers $P_{\mathrm{exc}}=50 \mu \mathrm{W}(\mathrm{g})$ and $P_{\mathrm{exc}}=750 \mu \mathrm{W}(\mathrm{h})$.

The short luminescence lifetime and the absorption cross-section of GaInP did not allow us to improve the far-field optical resolution using the STED concept. STED-light photons applied within the photoluminescence emission spectra caused an increase of the registered signal, indicating excitation of GaInP by the STED beam (data not shown). The high photostability of the GaInP photoluminescence encouraged us to use a complementary super-resolution method called ground state depletion (GSD) microscopy.

\subsubsection{Principles of GSD microscopy}

Ground state depletion (GSD) microscopy is a superresolution method complementary to STED microscopy, proposed by Stefan Hell in $1995^{45}$. Several years later, the applicability of this concept to organic fluorophores was proven experimentally ${ }^{112}$. The imaging scheme is very similar to STED 
microscopy, however, separation of the states is mediated by the process of optical excitation rather than by stimulated emission. The doughnut-shaped GSD beam is applied to deplete the ground state by optical shelving into an excited state, everywhere except at the targeted coordinate, where the beam intensity ideally equals zero. Originally, the GSD concept was proposed to utilize the transfer of fluorescent emitters from the ground state $S_{0}$ to a metastable long-lived dark state, as for instance the first excited triplet state $T_{1}$ naturally occurring in molecular dye probes. The molecular shelving into $T_{1}$ can be indirectly controlled by the excitation light, seeing that with each optical transition a small fraction of the excited molecules will eventually undergo intersystem crossing $\left(S_{0} \rightarrow S_{1} \rightsquigarrow T_{1}\right)$. Thus, the efficiency of this process depends on the intensity of the excitation GSD beam, which populates $S_{1}$ and effectively provides the ground state depletion. Upon separate illumination by a second weaker Gaussian-shaped probe beam operating at the same wavelength, molecules trapped in $T_{1}$ cannot generate fluorescence signal ('off' state). Only molecules nearby the coordinate targeted by the minimum of the GSD beam remain in the singlet system ('on' state) and can interact with the probing light. These emitters can be promoted to the first excited singlet state $\left(S_{0} \rightarrow S_{1}\right)$ and emit

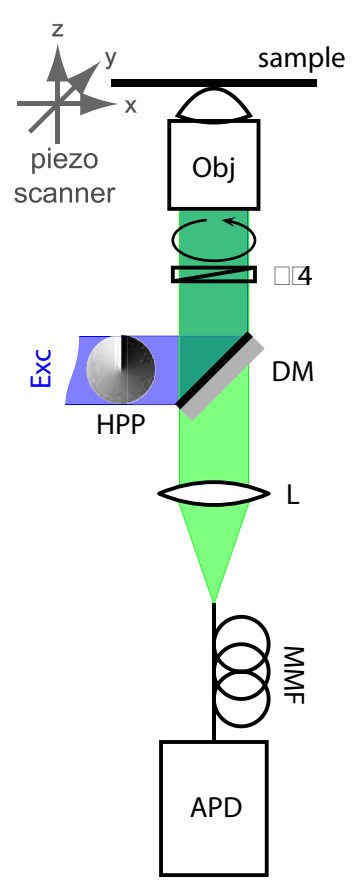

b

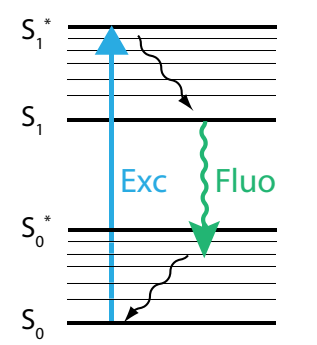

d

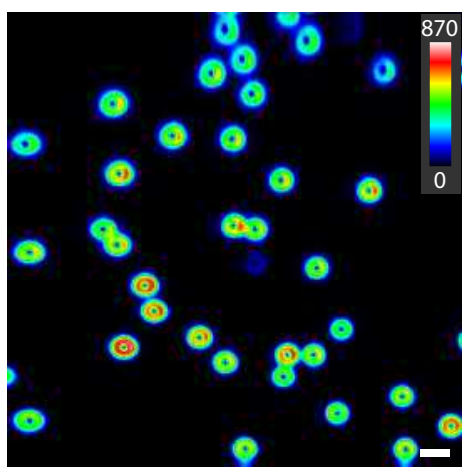

C

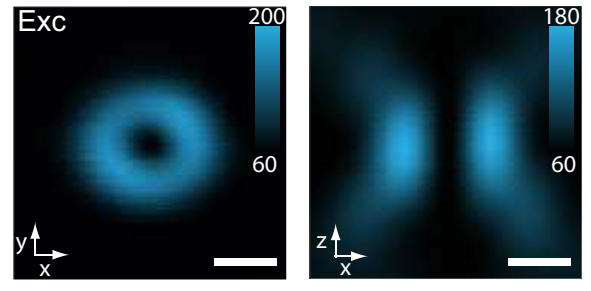

e

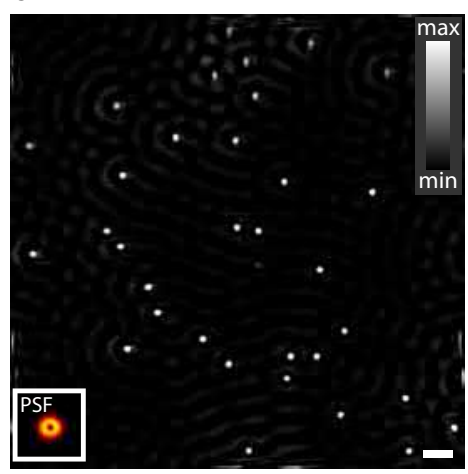

Figure 2.7: GSD nanoscopy. (a) Scheme of the GSD microscope. (Obj: objective lens, $\lambda / 4$ : quarter-wave plate, DM: dichroic mirror, HPP: helical phase plate, L: tube lens, MMF: multimode fiber, APD: avalanche photodiode). (b) Jabłoński diagram of an organic molecule showing the relevant electronic states: excitation (Exc) and spontaneous relaxation by fluorescence (Fluo). (c) Point spread function of the excitation beam in lateral $(x y)$ and axial $(x z)$ directions, as measured by scattering of a $80 \mathrm{~nm}$ gold bead. The scattering signal was measured on a photomultiplier tube (path not shown in (a)). The excitation wavelength was $720 \mathrm{~nm}$. (d) Raw data image of red-emitting fluorospheres $(715 \mathrm{~nm} / 755 \mathrm{~nm}$; maximal absorption and emission, respectively) with a diameter of $80 \mathrm{~nm}$. The average excitation power equals $\sim 500 \mu \mathrm{W}$ at $80 \mathrm{MHz}$. (e) Bead positions from image (d) restored by Wiener deconvolution with PSF (inset) estimated from a single bead image. Scale bars: $500 \mathrm{~nm}(\mathrm{c}), 1 \mu \mathrm{m}(\mathrm{d}, \mathrm{e})$. 
fluorescence signal. The volume of the effective 'on'-state emitters, which governs the resolution, can be significantly smaller than the diffraction limit, depending on the intensity of the GSD beam. The general concept of GSD microscopy is not restricted to the metastable dark state. Any excited molecular state which population depletes the ground state can be employed to increase the optical resolution. The very same idea was applied later in saturated structured illumination microscopy $(\mathrm{SSIM})^{113}$, where ground state depletion occurs by optical shelving of emitters in the first excited singlet state $S_{1}$ in a spatially parallelized manner. Employment of a long-lived dark state has the benefit of a more intuitive imaging modality which, unlike SSIM, does not necessitate any post-acquisition processing of data. The drawback is a limited speed, as for the point-to-point image acquisition markers trapped in the triplet state have to be able to return to the singlet system.

For some fluorophores, the triplet state cannot be efficiently populated due to a low probability of ISC. In this case, ground state depletion can be realized by shelving the emitters in the first excited singlet state $S_{1}$. Such a simple realization of the GSD concept is presented in Fig. 2.7. The experimental setup is very similar to that presented previously for STED microscopy (see Fig. 2.1). The doughnut-shaped excitation (GSD) focal spot is generated by a helical phase plate (HPP, Fig. 2.7a). To ensure a high-quality doughnut 'zero' and uniform interactions of photons with randomly oriented molecular dipoles, the beam features circular polarization in the focal plane. The image is created by point-by-point scanning and collection of the fluorescence signal map. Confocal detection is realized by a multimode optical fiber (MMF) to increase the image contrast in the axial direction. The possible molecular energy states are simplified to the ground state $S_{0}$ and the first excited singlet state $S_{1}$ (Fig. 2.7b). For an excitation rate significantly higher than the fluorescence rate, depletion of emitters from $S_{0}$ to $S_{1}$ is possible. At such high excitation intensities, the molecular response (fluorescence) to the excitation light is no longer linear. In this case, the fluorophore image represents the convolution of a molecular response function at the given excitation intensity and the diffractionlimited doughnut-shaped excitation focal spot (Fig. 2.7c). An example of a GSD raw-data image of red-emitting fluorescent beads is presented in Fig. 2.7d . The GSD variant discussed here has a 'negative' modality as the information about the molecular position is encoded in the minimum of the detected signal. The effective width of the dip, i.e. resolution, depends on the properties of the utilized optical transition. A more intuitive representation of the emitter positions can be retrieved after deconvolution (Fig. 2.7e, Wiener filtering). It is important to note that emitters at the doughnut crest will be subjected to significantly higher intensities than are necessary for shelving them in $S_{1}$. The success of this strategy in practice depends on how well the emitters can tolerate the light exposure. This method, even though experimentally simpler than STED, is usually strongly limited by photobleaching. Thus, the GSD concept has been realized only in special cases of low or negligible photobleaching, with NV centers in the diamond lattice ${ }^{9,114}$, some organic fluorophores at room temperature ${ }^{112}$ and organic fluorophores at liquid helium temperatures ${ }^{16}$. 


\subsubsection{Resolution scaling with excitation photon flux}

The resolution enhancement in the simplest version of GSD microscopy, which utilizes just the ground state $S_{0}$ and the first excited state $S_{1}$, can be derived from the population of $S_{1}$ described by

$$
\frac{d S_{1}}{d t}=\frac{\sigma_{\mathrm{exc}} I_{\mathrm{exc}}}{\hbar \omega} S_{0}-\gamma_{\mathrm{fl}} S_{1}
$$

where $\sigma_{\mathrm{exc}}$ is the optical cross-section for ground state absorption at the excitation wavelength, $I_{\mathrm{exc}}$ is the intensity of the excitation (GSD) beam, $\hbar \omega$ is the photon energy, $\gamma_{\mathrm{fl}}=\frac{1}{\tau_{\mathrm{fl}}}$ is the fluorescence rate with $\tau_{\mathrm{fl}}$ the fluorescence lifetime. If we assume that the molecules remain either in $S_{0}$ or in $S_{1}$ state we have $S_{0}+S_{1}=1$ and Equation 2.14 can be rewritten as a first-order differential equation

$$
\frac{d S_{1}}{d t}+\left(\frac{\sigma_{\mathrm{exc}} I_{\mathrm{exc}}}{\hbar \omega}+\gamma_{\mathrm{ff}}\right) S_{1}=\frac{\sigma_{\mathrm{exc}} I_{\mathrm{exc}}}{\hbar \omega} .
$$

Assuming the initial population in the excited state $S_{1}(t=0)=0$ and a rectangular excitation pulse with intensity $I_{\mathrm{exc}}$ acting in the time window $0 \leq t \leq \tau$, the population of $S_{1}$ just after excitation pulse is given by

$$
S_{1}(\tau)=\frac{w_{\mathrm{exc}}}{w_{\mathrm{exc}}+\gamma_{\mathrm{fl}}}\left(1-e^{-\left(w_{\mathrm{exc}}+\gamma_{\mathrm{fl}}\right) \tau}\right)
$$

where the excitation rate $w_{\text {exc }}=\frac{\sigma_{\text {exc }} I_{\text {exc }}}{\hbar \omega}$. For excitation pulses significantly longer than the excited-state lifetime $\left(\tau \gg \tau_{\mathrm{fl}}\right)$ or relatively high $w_{\mathrm{exc}}$, the equation simplifies to the steady-state population of $S_{1}$ given by

$$
S_{1}\left(I_{\mathrm{exc}}\right) \approx \frac{w_{\mathrm{exc}}}{w_{\mathrm{exc}}+\gamma_{\mathrm{fl}}}=\frac{I_{\mathrm{exc}}}{I_{\mathrm{exc}}+I_{\mathrm{s}}}
$$

a

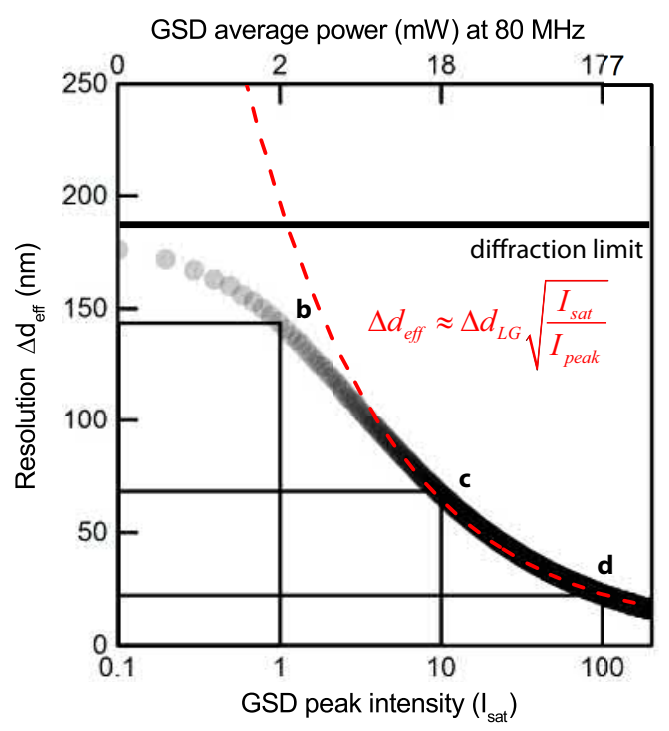

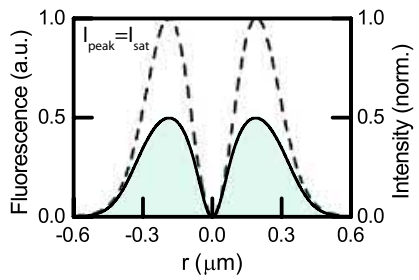

C
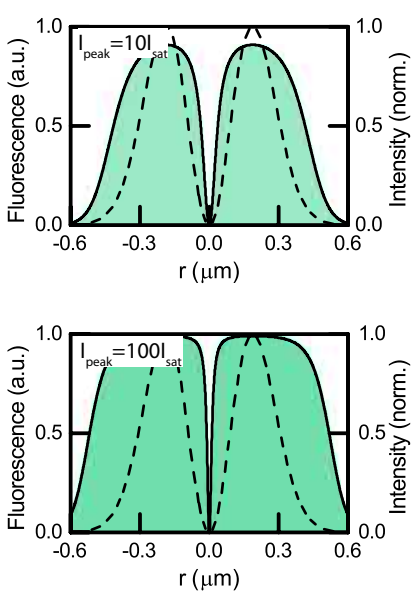

Figure 2.8: Resolution scaling in GSD microscopy. (a) Resolution scaling with GSD intensity for a pulse duration of $\tau_{\text {exc }}=3.5 \mathrm{~ns}$. Gray dots represent resolution calculated numerically from Equation 2.16 (inner dip diameter, FWHM); dashed red line is a fit of the given expression to the numerical data. (b-d) Excitation beam profile (dashed black line) and effective fluorescence (green) for the peak intensity $I_{\text {peak }}=I_{\text {sat }}$ in (b), $I_{\text {peak }}=10 I_{\text {sat }}$ in (c) and $I_{\text {peak }}=100 I_{\text {sat }}$ in (d). 
with characteristic intensity $I_{\mathrm{S}}=\frac{\gamma_{\mathrm{f}} \hbar \omega}{\sigma_{\mathrm{exc}}}$. The population of $S_{1}$ corresponds to the detected signal $\eta$ and the effective PSF is described by

$$
h_{\mathrm{eff}}(r) \propto \eta(r) \propto \frac{I_{\mathrm{exc}}(r)}{I_{\mathrm{exc}}(r)+I_{\mathrm{s}}}
$$

Nearby the targeted coordinate, the excitation intensity minimum can be approximated by the parabola (see Equation 2.4)

$$
I_{\mathrm{exc}}(r) \approx I_{\mathrm{peak}} \frac{2 e r^{2}}{w_{0}^{2}}
$$

where $w_{0}$ is the Laguerre-Gaussian beam parameter. The GSD resolution $\Delta d_{\mathrm{eff}}$ can be calculated from the FWHM of $h_{\mathrm{eff}}(r)$ as

$$
\Delta d_{\mathrm{eff}} \approx w_{0} \sqrt{\frac{I_{\mathrm{s}}}{I_{\mathrm{peak}}}}
$$

For an excitation wavelength of $700 \mathrm{~nm}$, an absorption cross-section of an organic fluorophore $\sigma_{\text {exc }}=1 \times 10^{-16} \mathrm{~cm}^{2}$ and a fluorescence lifetime $\tau_{\mathrm{fl}}=3.5 \mathrm{~ns}$, the characteristic intensity is $I_{\mathrm{s}}=811 \mathrm{~kW} / \mathrm{cm}^{2}$. The resolution gain in GSD microscopy as a function of the excitation peak intensity for an excitation pulse duration of $\tau_{\mathrm{exc}}=3.5 \mathrm{~ns}$ is shown in Fig. 2.8. A substantial resolution enhancement requires excitation intensities $I_{\text {peak }} \geq 10 I_{\text {s }}$.

\subsubsection{Influence of the excitation pulse duration on the GSD image}

In GSD microscopy, spontaneous relaxation competes with emitter shelving in the excited state. Efficient shelving necessitates a sufficient number of excitation photons applied ideally within a time shorter than the time for spontaneous relaxation $\tau_{l}$. Application of relatively long excitation (GSD) pulses results in an inefficient use of photons, as within the pulse duration some markers are able to relax to the ground state and need to be promoted again to the excited state. Therefore, long GSD pulses $\left(\tau_{\mathrm{exc}} \gg \tau_{l}\right)$ necessitate more photons to provide optical shelving and generate a higher fluorescence signal than short GSD pulses $\left(\tau_{\mathrm{exc}} \ll \tau_{l}\right)$.

As an example, saturation of the luminescence signal (fluorescence or photoluminescence) for a different excitation (GSD) pulse duration is considered. The emitter states are simplified to a 4-level system, including the ground state $E_{0}$, the excited state $E_{1}$ and the corresponding vibrational excited states $E_{0}^{*}, E_{1}^{*}$, as shown in Fig. 2.9. The dynamics of the system is described by the set of rate equations

$$
\begin{aligned}
\frac{\mathrm{d}}{\mathrm{d} t} E_{1}{ }^{*} & =w_{\mathrm{exc}}\left(E_{0}-E_{1}{ }^{*}\right)-\gamma_{\mathrm{vib}} E_{1}{ }^{*} \\
\frac{\mathrm{d}}{\mathrm{d} t} E_{1} & =\gamma_{\mathrm{vib}} E_{1}{ }^{*}-\gamma_{1} E_{1} \\
\frac{\mathrm{d}}{\mathrm{d} t} E_{0}{ }^{*} & =\gamma_{1} E_{1}-\gamma_{\mathrm{vib}} E_{0}{ }^{*} \\
\frac{\mathrm{d}}{\mathrm{d} t} E_{0} & =\gamma_{\mathrm{vib}} E_{0}{ }^{*}-w_{\mathrm{exc}}\left(E_{0}-E_{1}{ }^{*}\right)
\end{aligned}
$$

where $\gamma=\frac{1}{\tau}, \tau$ is the lifetime of the state, $w_{\mathrm{exc}}=\frac{\sigma_{\mathrm{exc}} I_{\mathrm{exc}}}{\hbar \omega}, \sigma_{\mathrm{exc}}$ is the excitation cross-section, $I_{\mathrm{exc}}$ is the excitation intensity and $\hbar \omega$ is the photon energy. Assuming a luminescence lifetime of $\tau_{l}=1 \mathrm{~ns}$, a fast vibrational relaxation of $\tau_{\mathrm{vib}}=0.001 \tau_{l}=1 \mathrm{ps}$, an excitation wavelength of $700 \mathrm{~nm}$ and an excitation cross-section of $\sigma_{\text {exc }}=1 \times 10^{16} \mathrm{~cm}^{2}$ numerical solution of the equations leads to the molecular response functions presented in Fig. 2.9a. The efficiency of optical shelving is the highest for the shortest pulse duration $\tau_{\mathrm{exc}}=0.1 \tau_{1}$. For an excitation pulse with a time-averaged power of $P_{\mathrm{exc}}=5 \mathrm{~mW}$ at $80 \mathrm{MHz}$ 
pulse repetition rate, the expected GSD resolution equals $\Delta d_{\mathrm{eff}}=88 \mathrm{~nm}$. Longer pulses provide higher signal and lower resolution for the same number of excitation photons provided. Fig. $2.9 \mathrm{~b}$ contains two images of the same GaInP segment incorporated in the GaP NW, at constant excitation time-averaged power for $\mathrm{CW}$ operation and pulsed excitation with $\sim 5 \mathrm{ps}$ pulse duration. Both signal and resolution are clearly dependent on the photon flux applied. The optimal imaging conditions depend on the dominant mechanism of light-induced damage. Photodamage proportional to the pulse energy (e.g., heat) favors relatively short excitation pulses $\left(\sim 0.1 \tau_{l}\right)$. Longer pulses should be applied in case of a high-order photodamage mechanism.

a
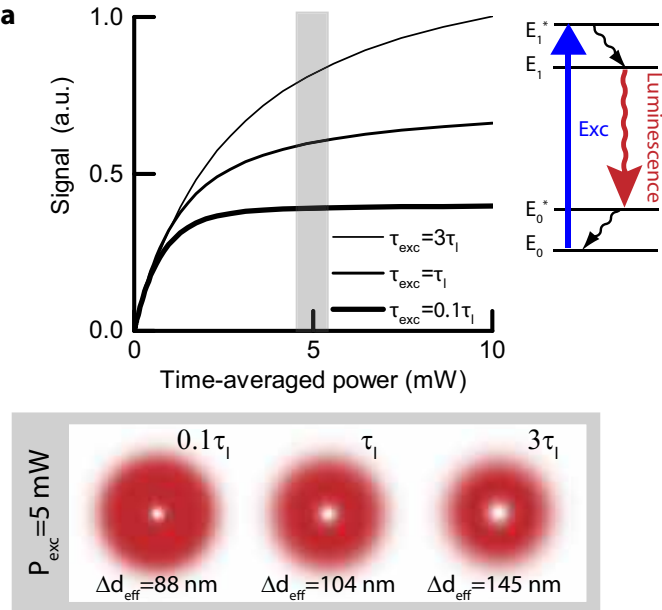
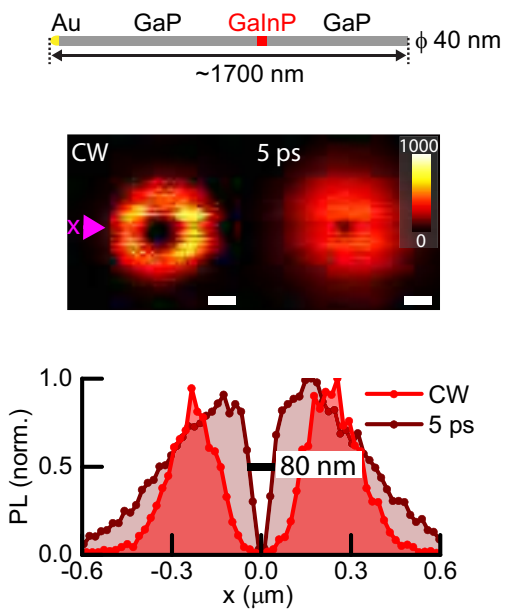

Figure 2.9: GSD imaging with different excitation pulse lengths. (a) (top) Luminescence signal saturation for different excitation pulses $\tau_{\text {exc }}$ (results of numerical integration of Eq. 2.21). The luminescence lifetime equals $\tau_{l}$. The inset represents the relevant energy states (see text). (bottom) Calculated GSD point spread functions for different excitation pulse lengths at a constant excitation power of $P_{\text {exc }}=5 \mathrm{~mW}$ at $80 \mathrm{MHz}$ repetition rate. (b) GaP-GaInP nanowire with a single GaInP segment imaged by continuous wave (CW) and pulsed excitation at a constant time-averaged power of $P_{\mathrm{exc}}=2 \mathrm{~mW}$. Excitation pulse duration: $\tau_{\mathrm{exc}}=5 \mathrm{ps}$; the pulse repetition rate: $80 \mathrm{MHz}$. The pink arrow indicates the direction $(x)$ of the selected intensity profile lines. Short excitation pulses provide better optical shelving, reflected by more narrow intensity dips and lower photoluminescence (PL) signal. Scale bars: $200 \mathrm{~nm}$.

\subsubsection{Restoration of emitter distributions from the GSD image}

The GSD microscopy discussed here provides raw-data images in a 'negative' modality, as the emitter positions are encoded in the detected signal minima. Mathematically, an image $g(r)$ formed in a raster scanning GSD microscope is described by the convolution of the effective PSF $h_{\mathrm{GSD}}(r)$ and the real emitter positions $f_{\mathrm{obj}}\left\{\delta\left(r-r_{j}\right)\right\}$ in the sample

$$
g(r)=h_{\mathrm{GSD}}(r) * f_{\mathrm{obj}}\left\{\delta\left(r-r_{j}\right)\right\} .
$$


where $r_{j}$ are the positions of the molecules and $*$ denotes the convolution operator. In GSD microscopy, the effective PSF $h_{\mathrm{GSD}}$ is dependent on the 'on' $\leftrightarrow$ 'off' state dynamics and given by

$$
h_{\mathrm{GSD}}\left(r, I_{\mathrm{exc}}\right)=h_{\mathrm{PSF}}(r) * h_{\mathrm{obj}}\left(\delta, I_{\mathrm{exc}}\right) \text {, }
$$

where $h_{\mathrm{PSF}}$ is the diffraction-limited (linear) excitation PSF and $h_{\mathrm{obj}}$ denotes the response function of the point-like emitter to the given excitation intensity $I_{\text {exc }}$.

A map of the emitters' distribution $f_{\text {obj }}(r)$ can be approximately restored from the raw-data GSD image $g(r)$ by deconvolution. A basic deconvolution algorithm exploits the fact that convolution corresponds to a multiplication in Fourier domain

$$
G_{\mathrm{GSD}}\left(v, I_{\mathrm{exc}}\right)=H_{\mathrm{GSD}}\left(v, I_{\mathrm{exc}}\right) F_{\mathrm{obj}}(v)
$$

where $G_{\mathrm{GSD}}, H_{\mathrm{GSD}}, F_{\mathrm{obj}}$ are the frequency representation of $g_{\mathrm{GSD}}, h_{\mathrm{GSD}}$ and $f_{\mathrm{obj}}$, respectively. The estimation of the exact object distribution can be described by

$$
f_{\mathrm{obj}}(r)=\mathscr{F}^{-1}\left\{F_{\mathrm{obj}}(v)\right\}=\mathscr{F}^{-1}\left\{\frac{G_{\mathrm{GSD}}\left(v, I_{\mathrm{exc}}\right)}{H_{\mathrm{GSD}}\left(v, I_{\mathrm{exc}}\right)}\right\},
$$

if $H_{\mathrm{GSD}}\left(v, I_{\mathrm{exc}}\right) \neq 0$ and $\mathscr{F}^{-1}$ denotes the inverse Fourier transform. It is very unlikely that $H_{\mathrm{GSD}}\left(v, I_{\mathrm{exc}}\right) \neq 0$ for all frequencies. Thus, presented above simple approach requires additional regularization parameters to minimize related artifacts. Moreover, both the estimated effective PSF $H_{\mathrm{GSD}}$ and registered image $G_{\mathrm{GSD}}$ are affected by presence of the measurement noise. Artifacts are visible as wave patterns ('ringing') around edges of the retrieved object positions (see restored image by Wiener deconvolution in Fig. 2.7e). Additional artifacts originate from the image boundary conditions. Signal discontinuities, between right-left and top-bottom borders of the image result in horizontal and vertical oscillations ('stripes') propagating trough the restored image. The most widely used deconvolution algorithms are Wiener filtering ${ }^{115}$ and Richardson-Lucy deconvolution ${ }^{116,117}$. 



\section{Chapter 3}

\section{Concluding discussion}

This research examined the impact of STED-light photon flux on the photobleaching of several commercially available organic dyes (ATTO647N, STAR635P, ATTO590, STAR580) in bulk experiments in thiodiglycol. The observed effects of STED-light induced photodamage varied among the investigated dyes. In general, we observed two characteristic bleaching regimes for a constant STED pulse energy, which roughly corresponds to a given factor of resolution enhancement: STED-intensitydependent photobleaching and STED-intensity-independent photobleaching. For ATTO647N the (bulk) photobleaching measurements revealed a single effective scaling with the STED intensity $\propto I_{\text {STED }}^{1.4}$, indicating a significant involvement of higher-excited molecular states in STED-light-induced damage. We proposed an intuitive photobleaching model for this dye, which provides a link from the bulk results to STED imaging conditions. This model can predict the attainable resolution and spatially-dependent photobleaching probability at different STED intensity levels. We showed that the high-order photobleaching component is minimized by increasing the STED pulse duration. To maintain the maximal resolution at a given STED pulse energy, the fluorescence detection gating has to be adjusted to the STED pulse duration. The optimal STED pulse length depends on the photobleaching scaling with the STED-light intensity and has to be optimized for a particular molecular probe and its microenvironment. These results rationalized the evident success of super-resolution imaging demonstrated with $\sim 1 \mathrm{~ns}$ STED pulses ${ }^{76}$. Moreover, this work revealed that recently demonstrated image acquisition strategies as MINFIELD ${ }^{96}$ or DyMIN ${ }^{97}$ could benefit from using relatively short STED pulses ( $\sim 10-100 \mathrm{ps})$, as the high-order photodamage component associated with excessive intensities present at the STED doughnut crest is already minimized. The application of shorter STED pulses would make it possible to collect more fluorescence signal at the coordinate targeted by the STED beam minimum, since detection gating is not necessary. Signal increase would shorten the necessary STED-light exposure time per pixel to acquire a suitable signal-to-noise ratio and therefore reduce photodamage.

Due to the complexity of the photobleaching process, we have limited the investigations to STED microscope operating at the commonly used de-excitation wavelength of $750 \mathrm{~nm}$. The choice of dyes (listed above) was restricted by the laser spectral properties. All measurements were conducted for a single environment of the molecular probes (thiodiglycol). These limitations should be addressed in follow-up studies. For example, optimization of the stimulated emission spectral window could 
diminish the damage mediated by particular optical transitions of the markers induced by absorption of STED-light photons. Unfortunately, the excited-state absorption spectra (in the singlet and triplet systems) at the STED wavelengths are often unknown for commonly used STED probes. Moreover, at high STED pulse energies, required for currently accessible resolutions of $\sim 20 \mathrm{~nm}$, a significant part of photobleaching is likely mediated by one-photon excitation of 'hot' molecules by photons from the STED beam. These events are not apparent in the STED experiment because of efficient de-excitation occurring in parallel, and also due to the confocalized detection system, which rejects a large part of the signal generated outside the targeted coordinate. The photobleaching initiated by one-photon absorption of ground-state molecules can be diminished by shifting the STED operation to a longer wavelength which is further away from the absorption maximum of the fluorophore. This approach reduces in the same time the stimulated emission cross-section. However, the roughly exponential decay of the thermally broadened red edge of the absorption spectral band is typically faster than the decay of the stimulated emission cross-section with STED wavelength. Additionally, the STED-light-mediated excitation of ground-state fluorophores is expected to be diminished at low temperatures. Low temperatures should freeze the Boltzmann broadening of the absorption spectral lines and allow to provide STED photons closer to the maximum of stimulated emission cross-section. The low-temperatures conditions, however, are less relevant in the biological context. Moreover, the photophysical properties of the molecules are different than at room temperature (e.g., longer lifetime of the lowest triplet state) and may require the modification of currently existing imaging schemes.

Our experiment did not discriminate singlet-system photobleaching from triplet-system photobleaching. Additional data on the photobleaching scaling in singlet and triplet systems with STED intensity would be of great interest. Techniques such as fluorescence correlation spectroscopy could bring new insights. The triplet-mediated damage can be significantly reduced by application of low repetition rate lasers ${ }^{29,30}$, fast scanning over a large field of view ${ }^{91}$ or chemical triplet quenchers ${ }^{20}$. After reduction of the triplet-mediated bleaching, any considerable high-order photobleaching could be lowered by application of relatively long STED pulses.

Further photobleaching studies of organic dyes and fluorescent proteins, especially in aqueous environments, could advance the capabilities of STED microscopy in biological systems. Research on the photobleaching of fluorescent proteins revealed the involvement of higher excited states in photobleaching under conventional confocal microscopy conditions ${ }^{89}$, indicating that long STED pulses could reduce photobleaching also for many of these emitters. However, little is known about the photobleaching of fluorescent proteins induced by light at the STED wavelengths, and further studies are necessary.

Reducing photobleaching of the fluorophores would make it possible to provide more STED photons to the sample and, therefore, increase the currently achievable resolution. Further increases require a better spatial definition of the STED doughnut minimum. At the currently applied STED energies, a significant part of the signal generated at the targeted coordinate is affected by the residual STED intensity in the minimum (causing stimulated emission). New experimental protocols to validate and improve the STED beam quality would, therefore, be valuable. 
The second part of this thesis demonstrated the use of GSD nanoscopy to image emissive GaInP segments within a non-emissive GaP nanowire. We have shown a 5-fold lateral resolution enhancement over conventional confocal microscopy. By using just a single laser beam at a wavelength of $700 \mathrm{~nm}$ (featuring low cellular toxicity) with a moderate power of $\sim 3 \mathrm{~mW}$ (pulse duration $\tau=5 \mathrm{ps}$, repetition rate $80 \mathrm{MHz}$ ), we resolved different geometries of GaP-GaInP nanowire barcodes with diameters of 20-80 nm. We put an emphasis on studying the influence of barcode geometry on the GSD image contrast. These findings should be relevant for applications of heterostructured nanowires in biological systems, as well as in other fields.

The combined super-resolution imaging of biological structures labeled with fluorophores and GSD imaging of heterostructured nanowires entails several challenges. A careful choice of the fluorescent markers is required. The GSD beam should ideally interact only with NWs and not with other molecular probes present in the sample. The spectral properties of the fluorophores should avoid one- and two-photon excitation events from GSD pulses of relatively short duration. Even in case of molecular excitation by GSD-light, the photoluminescence signal from nanowires can be decoupled from the fluorescence signal by lifetime differentiation using detection gating. The NWs investigated in this study featured emission lifetimes of $\sim 10 \mathrm{ps}$, which is several orders of magnitude shorter than the fluorescence lifetime of common fluorescent probes $(\sim 1-5 \mathrm{~ns})$. In addition, time gating can be employed to image fluorophores and nanowires with overlapping emission spectral composition.

The limiting factor to further resolution improvements in the GSD imaging demonstrated here is related to the light-induced damage of photoluminescent GaInP segments. At certain GSD powers of $\sim 3-7 \mathrm{~mW}$, we observed an abrupt and irreversible loss of the photoluminescence signal. The mechanism of photodamage is not yet understood. Further quantitative studies of the NWs' photodamage could enable the development of new procedures for nanostructure synthesis. For example, axial coating of GaP-GaInP nanowires by a higher bandgap semiconductor (core-shell engineering) could protect the GaInP electrons from chemical reactions with the local environment. Quantitative studies, however, require more detailed information on the GaInP crystal structure before and after the GSD-light-induced damage. Energy dispersive X-ray spectra and low-temperature photoluminescence spectra may bring new insights. Such additional data would make it possible to study the influence of dopants and surface defects on GaInP photostability for different nanowire geometries. In the current work, we were not able to address these issues, as even for identical growth parameters the consecutive GaInP segments within a single nanowire exhibited significant differences in the photoluminescence strength, likely indicating a difference in the segments' material composition.

The imaging of GaP-GaInP nanowires with diameters $<20 \mathrm{~nm}$ constitutes another challenge. With the decrease of nanowire diameter we noticed an increase of random fluctuations of the emitted photoluminescence signal. For $\sim 10 \mathrm{~nm}$ diameter NWs, we have observed relatively strong signal intermittencies. This severe drawback did not allow us to resolve the GaInP segments using GSD nanoscopy in this case. Signal fluctuations can be possibly reduced by core-shell engineering, similarly as it has been done to reduce blinking in quantum dots ${ }^{103,104}$. As signal flickering occurs when the 
nanostructure size is approaching the characteristic excition size (Bohr radius) of a semiconductor material, imaging of thinner nanowires could be realized by choosing a NW material with a smaller exciton Bohr radius.

The point-by-point image acquisition scheme applied here is not time-efficient for large fields of view. However, the moderate power requirements of GSD imaging would allow for parallelization of the image collection, in a similar manner to the scheme demonstrated for RESOLFT nanoscopy ${ }^{60}$.

Many applications involve nanowire arrays, where nanowires are oriented in parallel to the objective axis of the microscopy system. An axial resolution enhancement for heterostructured nanowires has not been demonstrated yet, however, this should be relatively straightforward for GSD nanoscopy, as the strategy can be replicated from that previously applied in STED nanoscopy ${ }^{23}$. 


\section{Bibliography}

[1] Hooke, R. Micrographia, or, Some physiological descriptions of minute bodies made by magnifying glasses: With observations and inquiries thereupon. (Printed by J. Martyn and J. Allestry, 1665).

[2] Abbe, E. Beiträge zur Theorie des Mikroskops und der mikroskopischen Wahrnehmung. Archiv für mikroskopische Anatomie 9, 413-418 (1873).

[3] Hell, S. W. Far-field optical nanoscopy. Science 316, 1153-1158 (2007).

[4] Huang, B., Babcock, H. \& Zhuang, X. Breaking the diffraction barrier: Super-resolution imaging of cells. Cell 143, 1047-1058 (2010).

[5] Dyba, M. \& Hell, S. W. Focal spots of size $\lambda / 23$ open up far-field florescence microscopy at $33 \mathrm{~nm}$ axial resolution. Physical Review Letters 88, 163901 (2002).

[6] Rust, M. J., Bates, M. \& Zhuang, X. Sub-diffraction-limit imaging by stochastic optical reconstruction microscopy (STORM). Nature Methods 3, 793-796 (2006).

[7] Betzig, E. et al. Imaging intracellular fluorescent proteins at nanometer resolution. Science 313, 1642-1645 (2006).

[8] Hess, S. T., Girirajan, T. P. K. \& Mason., M. D. Ultra-high resolution imaging by fluorescence photoactivation localization microscopy. Biophysical Journal 91, 4258-4272 (2006).

[9] Rittweger, E., Wildanger, D. \& Hell, S. W. Far-field fluorescence nanoscopy of diamond color centers by ground state depletion. Europhysics Letters 86, 14001 (2009).

[10] Dertinger, T., Colyer, R., Iyer, G., Weiss, S. \& Enderlein, J. Fast, background-free, (3D) super-resolution optical fluctuation imaging (SOFI). Proceedings of the National Academy of Sciences 106, 22287-22292 (2009).

[11] Hoyer, P., Staudt, T., Engelhardt, J. \& Hell, S. W. Quantum Dot Blueing and Blinking Enables Fluorescence Nanoscopy. Nano Letters 11, 245-250 (2011).

[12] Hanne, J. et al. STED nanoscopy with fluorescent quantum dots. Nature Communications 6, 7127 (2015). 
[13] Kolesov, R. et al. Super-resolution upconversion microscopy of praseodymium-doped yttrium aluminum garnet nanoparticles. Physical Review B 84, 153413 (2011).

[14] Liu, Y. et al. Amplified stimulated emission in upconversion nanoparticles for super-resolution nanoscopy. Nature 543, 229-233 (2017).

[15] Wildanger, D. et al. Solid immersion facilitates fluorescence microscopy with nanometer resolution and sub-ångström emitter localization. Advanced Materials 24, OP309-OP313 (2012).

[16] Yang, B., Trebbia, J.-B., Baby, R., Tamarat, P. \& Lounis, B. Optical nanoscopy with excited state saturation at liquid helium temperatures. Nature Photonics 9, 658-662 (2015).

[17] Weisenburger, S. et al. Cryogenic optical localization provides (3D) protein structure data with angstrom resolution. Nature Methods 14, 141-144 (2017).

[18] Hell, S. W. \& Wichmann, J. Breaking the diffraction resolution limit by stimulated emission: stimulated-emission-depletion fluorescence microscopy. Optics Letters 19, 780-782 (1994).

[19] Song, L., Hennink, E., Young, I. \& Tanke, H. Photobleaching kinetics of fluorescein in quantitative fluorescence microscopy. Biophysical Journal 68, 2588-2600 (1995).

[20] Song, L., Varma, C. A., Verhoeven, J. W. \& Tanke, H. J. Influence of the triplet excited state on the photobleaching kinetics of fluorescein in microscopy. Biophysical Journal 70, 2959-2968 (1996).

[21] Patterson, G. H. \& Piston, D. W. Photobleaching in two-photon excitation microscopy. Biophysical Journal 78, 2159-2162 (2000).

[22] Eggeling, C., Volkmer, A. \& Seidel, C. A. M. Molecular photobleaching kinetics of rhodamine $6 \mathrm{~g}$ by one- and two-photon induced confocal fluorescence microscopy. ChemPhysChem $\mathbf{6}$, 791-804 (2005).

[23] Klar, T. A., Jakobs, S., Dyba, M., Egner, A. \& Hell, S. W. Fluorescence microscopy with diffraction resolution barrier broken by stimulated emission. Proceedings of the National Academy of Sciences 97, 8206-8210 (2000).

[24] Westphal, V. \& Hell, S. W. Nanoscale resolution in the focal plane of an optical microscope. Physical Review Letters 94, 143903 (2005).

[25] Willig, K. I., Harke, B., Medda, R. \& Hell, S. W. STED microscopy with continuous wave beams. Nature Methods 4, 915-918 (2007).

[26] Vicidomini, G. et al. Sharper low-power STED nanoscopy by time gating. Nature Methods $\mathbf{8}$, 571-573 (2011). 
[27] Hell, S. W. et al. Stimulated emission on microscopic scale: Light quenching of Pyridine 2 using a Ti:sapphire laser. Journal of Microscopy 180, RP1-RP2 (1995).

[28] Dyba, M. \& Hell, S. W. Photostability of a fluorescent marker under pulsed excited-state depletion through stimulated emission. Applied Optics 42, 5123 (2003).

[29] Donnert, G. et al. Macromolecular-scale resolution in biological fluorescence microscopy. Proceedings of the National Academy of Sciences 103, 11440-11445 (2006).

[30] Donnert, G., Eggeling, C. \& Hell, S. W. Major signal increase in fluorescence microscopy through dark-state relaxation. Nature Methods 4, 81-86 (2006).

[31] Hotta, J. et al. Spectroscopic rationale for efficient stimulated-emission depletion microscopy fluorophores. Journal of the American Chemical Society 132, 5021-5023 (2010).

[32] Han, M., Gao, X., Su, J. Z. \& Nie, S. Quantum-dot-tagged microbeads for multiplexed optical coding of biomolecules. Nature Biotechnology 19, 631-635 (2001).

[33] Dahan, M. Diffusion dynamics of glycine receptors revealed by single-quantum dot tracking. Science 302, 442-445 (2003).

[34] Jovin, T. M. Quantum dots finally come of age. Nature Biotechnology 21, 32-33 (2003).

[35] Barroso, M. M. Quantum dots in cell biology. Journal of Histochemistry \& Cytochemistry 59, 237-251 (2011).

[36] Patolsky, F. et al. Detection, stimulation, and inhibition of neuronal signals with high-density nanowire transistor arrays. Science 313, 1100-1104 (2006).

[37] Robinson, J. T. et al. Vertical nanowire electrode arrays as a scalable platform for intracellular interfacing to neuronal circuits. Nature Nanotechnology 7, 180-184 (2012).

[38] Suyatin, D. B. et al. Gallium phosphide nanowire arrays and their possible application in cellular force investigations. Journal of Vacuum Science \& Technology B: Microelectronics and Nanometer Structures 27, 3092 (2009).

[39] Hällström, W. et al. Fifteen-piconewton force detection from neural growth cones using nanowire arrays. Nano Letters 10, 782-787 (2010).

[40] Kim, W., Ng, J. K., Kunitake, M. E., Conklin, B. R. \& Yang, P. Interfacing silicon nanowires with mammalian cells. Journal of the American Chemical Society 129, 7228-7229 (2007).

[41] Shalek, A. K. et al. Vertical silicon nanowires as a universal platform for delivering biomolecules into living cells. Proceedings of the National Academy of Sciences of the United States of America 107, 1870-1875 (2010).

[42] Berthing, T. et al. Intact mammalian cell function on semiconductor nanowire arrays: New perspectives for cell-based biosensing. Small 7, 640-647 (2011). 
[43] Mumm, F., Beckwith, K. M., Bonde, S., Martinez, K. L. \& Sikorski, P. A transparent nanowirebased cell impalement device suitable for detailed cell nanowire interaction studies. Small $\mathbf{9}$, 263-272 (2013).

[44] Adolfsson, K. et al. Fluorescent nanowire heterostructures as a versatile tool for biology applications. Nano Letters 13, 4728-4732 (2013).

[45] Hell, S. W. \& Kroug, M. Ground-state-depletion fluorescence microscopy: A concept for breaking the diffraction resolution limit. Applied Physics B 60, 495-497 (1995).

[46] Hell, S. W., Dyba, M. \& Jakobs, S. Concepts for nanoscale resolution in fluorescence microscopy. Current Opinion in Neurobiology 14, 599 - 609 (2004).

[47] Sharonov, A. \& Hochstrasser, R. M. Wide-field subdiffraction imaging by accumulated binding of diffusing probes. Proceedings of the National Academy of Sciences 103, 18911-18916 (2006).

[48] Fölling, J. et al. Fluorescence nanoscopy by ground-state depletion and single-molecule return. Nature Methods 5, 943-945 (2008).

[49] Moerner, W. E. \& Fromm, D. P. Methods of single-molecule fluorescence spectroscopy and microscopy. Review of Scientific Instruments 74, 3597-3619 (2003).

[50] Heisenberg, W. The Physical Principles of the Quantum Theory. Dover Books on Physics and Chemistry (1949).

[51] Gordon, M. P., Ha, T. \& Selvin, P. R. Single-molecule high-resolution imaging with photobleaching. Proceedings of the National Academy of Sciences of the United States of America 101, 6462-6465 (2004).

[52] Qu, X., Wu, D., Mets, L. \& Scherer, N. F. Nanometer-localized multiple single-molecule fluorescence microscopy. Proceedings of the National Academy of Sciences of the United States of America 101, 11298-11303 (2004).

[53] Lidke, K. A., Rieger, B., Jovin, T. M. \& Heintzmann, R. Superresolution by localization of quantum dots using blinking statistics. Optics Express 13, 7052-7062 (2005).

[54] Dickson, R. M., Cubitt, A. B., Tsien, R. Y. \& Moerner, W. E. On/off blinking and switching behaviour of single molecules of green fluorescent protein. Nature 388, 355-358 (1997).

[55] Patterson, G. H. \& Lippincott-Schwartz, J. A photoactivatable GFP for selective photolabeling of proteins and cells. Science 297, 1873-1877 (2002).

[56] Habuchi, S. et al. Reversible single-molecule photoswitching in the GFP-like fluorescent protein Dronpa. Proceedings of the National Academy of Sciences of the United States of America 102, 9511-9516 (2005). 
[57] Bates, M., Blosser, T. R. \& Zhuang, X. Short-range spectroscopic ruler based on a singlemolecule optical switch. Physical Review Letters 94, 108101 (2005).

[58] Balzarotti, F. et al. Nanometer resolution imaging and tracking of fluorescent molecules with minimal photon fluxes. Science 355, 606-612 (2016).

[59] Bingen, P., Reuss, M., Engelhardt, J. \& Hell, S. W. Parallelized STED fluorescence nanoscopy. Optics Express 19, 23716-23726 (2011).

[60] Chmyrov, A. et al. Nanoscopy with more than 100,000 'doughnuts'. Nature Methods 10, 737-740 (2013).

[61] van Oijen, A., Köhler, J., Schmidt, J., Müller, M. \& Brakenhoff, G. 3-Dimensional superresolution by spectrally selective imaging. Chemical Physics Letters 292, 183-187 (1998).

[62] Speidel, M., Jonáš, A. \& Florin, E.-L. Three-dimensional tracking of fluorescent nanoparticles with subnanometer precision by use of off-focus imaging. Optics Letters 28, 69-71 (2003).

[63] Kao, H. \& Verkman, A. Tracking of single fluorescent particles in three dimensions: use of cylindrical optics to encode particle position. Biophysical Journal 67, 1291-1300 (1994).

[64] Huang, B., Wang, W., Bates, M. \& Zhuang, X. Three-dimensional super-resolution imaging by stochastic optical reconstruction microscopy. Science 319, 810-813 (2008).

[65] Ram, S., Chao, J., Prabhat, P., Ward, E. S. \& Ober, R. J. A novel approach to determining the three-dimensional location of microscopic objects with applications to 3D particle tracking. In Three-Dimensional and Multidimensional Microscopy: Image Acquisition and Processing XIV (SPIE, 2007).

[66] Izeddin, I. et al. PSF shaping using adaptive optics for three-dimensional single-molecule super-resolution imaging and tracking. Optics Express 20, 4957-4967 (2012).

[67] Pavani, S. R. P. et al. Three-dimensional, single-molecule fluorescence imaging beyond the diffraction limit by using a double-helix point spread function. Proceedings of the National Academy of Sciences 106, 2995-2999 (2009).

[68] Zhang, Z., Kenny, S. J., Hauser, M., Li, W. \& Xu, K. Ultrahigh-throughput single-molecule spectroscopy and spectrally resolved super-resolution microscopy. Nature Methods 12, 935-938 (2015).

[69] Sahl, S. J., Hell, S. W. \& Jakobs, S. Fluorescence nanoscopy in cell biology. Nature Reviews Molecular Cell Biology 18, 685-701 (2017).

[70] Grosse, L. et al. Bax assembles into large ring-like structures remodeling the mitochondrial outer membrane in apoptosis. The EMBO Journal 35, 402-413 (2016). 
[71] Xu, K., Zhong, G. \& Zhuang, X. Actin, spectrin, and associated proteins form a periodic cytoskeletal structure in axons. Science 339, 452-456 (2012).

[72] Hess, S. T. et al. Dynamic clustered distribution of hemagglutinin resolved at $40 \mathrm{~nm}$ in living cell membranes discriminates between raft theories. Proceedings of the National Academy of Sciences 104, 17370-17375 (2007).

[73] Iketaki, Y., Watanabe, T., Bokor, N. \& Fujii, M. Investigation of the center intensity of first- and second-order Laguerre-Gaussian beams with linear and circular polarization. Optics Letters 32, 2357-2359 (2007).

[74] Kozawa, Y. \& Sato, S. Sharper focal spot formed by higher-order radially polarized laser beams. Journal of the Optical Society of America A 24, 1793-1798 (2007).

[75] Reuss, M., Engelhardt, J. \& Hell, S. W. Birefringent device converts a standard scanning microscope into a STED microscope that also maps molecular orientation. Optics Express 18, 1049-1058 (2010).

[76] Göttfert, F. et al. Coaligned dual-channel STED nanoscopy and molecular diffusion analysis at 20 nm resolution. Biophysical Journal 105, L01-L03 (2013).

[77] Moffitt, J. R., Osseforth, C. \& Michaelis, J. Time-gating improves the spatial resolution of STED microscopy. Optics Express 19, 4242-4254 (2011).

[78] Wilson, T., Shepparu, C. J. R. \& Löschke, K. Theory and Practice of Scanning Optical Microscopy (Academic Press, 1984).

[79] Dehez, H., Piché, M. \& Koninck, Y. D. Resolution and contrast enhancement in laser scanning microscopy using dark beam imaging. Optics Express 21, 15912-15925 (2013).

[80] Richards, B. \& Wolf, E. Electromagnetic diffraction in optical systems, II. Structure of the image field in an aplanatic system. Proceedings of the Royal Society of London A: Mathematical, Physical and Engineering Sciences 253, 358-379 (1959).

[81] Sheppard, C. J. R. \& Wilson, T. The image of a single point in microscopes of large numerical aperture. Proceedings of the Royal Society of London A: Mathematical, Physical and Engineering Sciences 379, 145-158 (1982).

[82] Harke, B. et al. Resolution scaling in STED microscopy. Optics Express 16, 4154-4162 (2008).

[83] Calvert, J. G. \& Jr., J. N. P. Photochemistry (John Wiley \& Sons, 1966).

[84] Kaminow, I. P., Stulz, L. W., Chandross, E. A. \& Pryde, C. A. Photobleaching of organic laser dyes in solid matrices. Applied Optics 11, 1563 (1972).

[85] Eggeling, C., Widengren, J., Rigler, R. \& Seidel, C. A. M. Photobleaching of fluorescent dyes under conditions used for single-molecule detection: evidence of two-step photolysis. Analytical Chemistry 70, 2651-2659 (1998). 
[86] Niesner, R., Roth, W. \& Gericke, K.-H. Photophysical aspects of single-molecule detection by two-photon excitation with consideration of sequential pulsed illumination. ChemPhysChem $\mathbf{5}$, 678-687 (2004).

[87] Zheng, Q. et al. Ultra-stable organic fluorophores for single-molecule research. Chemical Society Reviews 43, 1044-1056 (2014).

[88] Chen, T.-S., Zeng, S.-Q., Luo, Q.-M., Zhang, Z.-H. \& Zhou, W. High-order photobleaching of green fluorescent protein inside live cells in two-photon excitation microscopy. Biochemical and Biophysical Research Communications 291, 1272-1275 (2002).

[89] Cranfill, P. J. et al. Quantitative assessment of fluorescent proteins. Nature Methods 13, 557-562 (2016).

[90] Kalies, S., Kuetemeyer, K. \& Heisterkamp, A. Mechanisms of high-order photobleaching and its relationship to intracellular ablation. Biomedical Optics Express 2, 805-816 (2011).

[91] Wu, Y. et al. Resonant scanning with large field of view reduces photobleaching and enhances fluorescence yield in STED microscopy. Scientific Reports 5, 14766 (2015).

[92] Schneider, J. et al. Ultrafast, temporally stochastic STED nanoscopy of millisecond dynamics. Nature Methods 12, 827-830 (2015).

[93] Scott, R. H. \& Balasubramanian, S. Properties of fluorophores on solid phase resins; implications for screening, encoding and reaction monitoring. Bioorganic \& Medicinal Chemistry Letters 7, 1567-1572 (1997).

[94] Yan, B., Martin, P. C. \& Lee, J. Single-bead fluorescence microspectroscopy: detection of self-quenching in fluorescence-labeled resin beads. Journal of Combinatorial Chemistry $\mathbf{1}$, 78-81 (1999).

[95] Staudt, T. et al. Far-field optical nanoscopy with reduced number of state transition cycles. Optics Express 19, 5644-5657 (2011).

[96] Göttfert, F. et al. Strong signal increase in STED fluorescence microscopy by imaging regions of subdiffraction extent. Proceedings of the National Academy of Sciences 114, 2125-2130 (2017).

[97] Heine, J. et al. Adaptive-illumination STED nanoscopy. Proceedings of the National Academy of Sciences 114, 9797-9802 (2017).

[98] Danzl, J. G. et al. Coordinate-targeted fluorescence nanoscopy with multiple off states. Nature Photonics 10, 122-128 (2016).

[99] Hofmann, M., Eggeling, C., Jakobs, S. \& Hell, S. W. Breaking the diffraction barrier in fluorescence microscopy at low light intensities by using reversibly photoswitchable proteins. Proceedings of the National Academy of Sciences 102, 17565-17569 (2005). 
[100] Swaminathan, R., Hoang, C. \& Verkman, A. Photobleaching recovery and anisotropy decay of green fluorescent protein GFP-S65T in solution and cells: cytoplasmic viscosity probed by green fluorescent protein translational and rotational diffusion. Biophysical Journal $\mathbf{7 2}$, 1900-1907 (1997).

[101] Zhou, X. X. \& Lin, M. Z. Photoswitchable fluorescent proteins: ten years of colorful chemistry and exciting applications. Current Opinion in Chemical Biology 17, 682-690 (2013).

[102] Bradac, C. et al. Observation and control of blinking nitrogen-vacancy centres in discrete nanodiamonds. Nature Nanotechnology 5, 345-349 (2010).

[103] Galland, C. et al. Two types of luminescence blinking revealed by spectroelectrochemistry of single quantum dots. Nature 479, 203-207 (2011).

[104] Efros, A. L. \& Nesbitt, D. J. Origin and control of blinking in quantum dots. Nature Nanotechnology 11, 661-671 (2016).

[105] Lu, W. \& Lieber, C. M. Semiconductor nanowires. Journal of Physics D: Applied Physics 39, R387-R406 (2006).

[106] Patolsky, F. \& Lieber, C. M. Nanowire nanosensors. Materials Today 8, 20-28 (2005).

[107] Christelle, N. P. Interactions between semiconductor nanowires and living cells. Journal of Physics: Condensed Matter 27, 233103 (2015).

[108] Nel, A. Toxic potential of materials at the nanolevel. Science 311, 622-627 (2006).

[109] Adolfsson, K. GaP and GaInP nanowires as model particles for in vivo fiber toxicity studies. Ph.D. thesis, Lund University (2017).

[110] Lee, J.-W., Schremer, A. T., Fekete, D., Shealy, J. R. \& Ballantyne, J. M. Growth of direct bandgap GalnP quantum dots on GaP substrates. Journal of Electronic Materials 26, 1199-1204 (1997).

[111] Brambilla, G. et al. Optical fiber nanowires and microwires: fabrication and applications. Advances in Optics and Photonics 1, 107-161 (2009).

[112] Bretschneider, S., Eggeling, C. \& Hell, S. W. Breaking the diffraction barrier in fluorescence microscopy by optical shelving. Physical Review Letters 98, 218103 (2007).

[113] Gustafsson, M. G. L. Nonlinear structured-illumination microscopy: Wide-field fluorescence imaging with theoretically unlimited resolution. Proceedings of the National Academy of Sciences of the United States of America 102, 13081-13086 (2005).

[114] Han, K. Y., Kim, S. K., Eggeling, C. \& Hell, S. W. Metastable dark states enable ground state depletion microscopy of nitrogen vacancy centers in diamond with diffraction-unlimited resolution. Nano Letters 10, 3199-3203 (2010). 
[115] Wiener, N. Extrapolation, interpolation, and smoothing of stationary time series, with engineering applications. (Technology Press of the Massachusetts Institute of Technology, 1950).

[116] Richardson, W. H. Bayesian-based iterative method of image restoration. Journal of the Optical Society of America 62, 55-59 (1972).

[117] Lucy, L. B. An iterative technique for the rectification of observed distributions. The Astronomical Journal 79, 745-754 (1974). 

Chapter 4

Photobleaching in STED nanoscopy and its dependence on the photon flux applied for reversible silencing of the fluorophore 

Received: 10 May 2017

Accepted: 1 August 2017

Published online: 12 September 2017

\section{Photobleaching in STED nanoscopy and its dependence on the photon flux applied for reversible silencing of the fluorophore}

\author{
Joanna Oracz $\mathbb{D}^{1,2}$, Volker Westphal ${ }^{1}$, Czesław Radzewicz ${ }^{2}$, Steffen J. Sahl ${ }^{1}$ \& Stefan W. Hell ${ }^{1,3}$
}

In STED (stimulated emission depletion) nanoscopy, the resolution and signal are limited by the fluorophore de-excitation efficiency and photobleaching. Here, we investigated their dependence on the pulse duration and power of the applied STED light for the popular $750 \mathrm{~nm}$ wavelength. In experiments with red- and orange-emitting dyes, the pulse duration was varied from the subpicosecond range up to continuous-wave conditions, with average powers up to $200 \mathrm{~mW}$ at $80 \mathrm{MHz}$ repetition rate, i.e. peak powers up to $1 \mathrm{~kW}$ and pulse energies up to $2.5 \mathrm{~nJ}$. We demonstrate the dependence of bleaching on pulse duration, which dictates the optimal parameters of how to deliver the photons required for transient fluorophore silencing. Measurements with the dye ATTO647N reveal that the bleaching of excited molecules scales with peak power with a single effective order $\sim 1.4$. This motivates peak power reduction while maintaining the number of STED-light photons, in line with the superior resolution commonly achieved for nanosecond STED pulses. Other dyes (ATTO590, STAR580, STAR635P) exhibit two distinctive bleaching regimes for constant pulse energy, one with strong dependence on peak power, one nearly independent. We interpret the results within a photobleaching model that guides quantitative predictions of resolution and bleaching.

Stimulated emission depletion (STED) nanoscopy ${ }^{1}$ is now a well-established method which allows minimally invasive optical access to length scales of tens of nanometers, in fixed and living biological specimens ${ }^{2}$. In addition to the focused excitation beam of confocal laser scanning microscopy, a now widespread implementation of STED uses a doughnut-shaped de-excitation profile to keep fluorophore molecules outside the central nanoscale region effectively in the ground state for the duration of detection at a given scan coordinate. The STED light's role is therefore to transiently keep molecules non-fluorescent and briefly switch fluorescence "off". Two key strengths of STED nanoscopy in comparison to other super-resolution fluorescence methods are the universality of its resolution-enabling fluorescence off-switching mechanism, allowing STED to operate well with many different markers, and its ability to image fast dynamics at video rate and beyond ${ }^{3-5}$. The presence of sufficiently intense STED light guarantees the off-switching of fluorophores by stimulated emission, except at the central intensity minimum (ideally, a zero of intensity) of the doughnut, where the "on"-state is established. The spatial resolution in STED nanoscopy is consequently no longer limited by the extent of the focal intensity distribution set by diffraction, but depends on the creation of the "on"-"off" state difference among fluorophores by switching.

To record a super-resolved image by scanning, the fluorescence ability of fluorophores at positions near the targeted coordinate (region of the doughnut) has to be switched off many times anew, with typical peak intensities present in the off-switching profile on the order of $\sim 1 \mathrm{GW} / \mathrm{cm}^{2}$ (ref. 6). This high irradiance at the doughnut crest poses challenges, notably the photobleaching of fluorescent markers, in particular of the widely employed small-molecule organic dyes. Several chemical approaches have been demonstrated to partially mitigate this problem, including the development of new fluorescent dyes ${ }^{7,8}$, decreasing the concentration of free oxygen to minimize destructive chemical reactions ${ }^{9}$, quenching of the triplet state ${ }^{10}$, as well as different optical strategies,

${ }^{1}$ Max Planck Institute for Biophysical Chemistry, Department of NanoBiophotonics, Am Fassberg 11, 37077, Göttingen, Germany. ${ }^{2}$ University of Warsaw, Faculty of Physics, Pastera 5, 02-093, Warsaw, Poland. ${ }^{3}$ Max Planck Institute for Medical Research, Department of Optical Nanoscopy, Jahnstr. 29, 69120, Heidelberg, Germany. Correspondence and requests for materials should be addressed to J.O. (email: joracz@mpibpc.mpg.de) or S.W.H. (email: shell@mpibpc.mpg.de) 
such as: use of longer STED pulses to avoid multiphoton excitation ${ }^{11}$, fast scanners or lasers with low repetition rates to allow triplet state relaxation between two successive pulses ${ }^{12-14}$, or other approaches which minimize unnecessary exposure to the intense light ${ }^{15-17}$.

Despite the success of STED methods with different implementations and laser schemes - from pulsed Ti:sapphire lasers to continuous wave (CW) diode lasers and, more recently, nanosecond pulsed fiber lasers - the full potential of the STED concept has not been realized. While $<10 \mathrm{~nm}$ resolution has been achieved for ultrastable fluorescent color centers in diamond ${ }^{18,19}$, closing the gap to molecular resolution for small organic fluorophores that are subject to bleaching remains a challenge. The question which experimental parameters hold the key to achieving routine STED resolution levels well below the present 20-30 nm mark remains unanswered. One main reason for this is that it has been difficult to compare different, disparate experiments and implementations of STED, and conclude on the importance of individual parameters. In general, simultaneous determination of photobleaching impact and the resolution achieved is challenged by the fact that higher resolution is typically associated with more pronounced photobleaching. This makes repetitive measurements to gain the necessary statistics on both quantities difficult to acquire. The situation is even more complicated in biological specimens, which often feature variable local environments and randomly varying fluorophore distributions. Moreover, the non-uniform intensity of the doughnut-shaped STED profile in the focal plane causes a spatial dependence of the STED-beam-induced photobleaching. This spatial variability of bleaching is integrated over in the signal collection, thus limiting definitive conclusions.

Here, we report a detailed study of molecular photobleaching and de-excitation efficiency in STED nanoscopy with organic fluorophores. The contribution to signal related to the unwanted absorption of photons from the STED beam (STED-light-induced fluorescence) is also systematically assessed. We investigated how the intensity impacts bleaching over a large range of pulse peak powers and energies. Our study was enabled by thorough experimental design to reduce the variability of conditions and error of measurement. Concretely, to obtain quantitative results on the impact of bleaching as a function of the flux of STED-light photons, it was necessary to perform measurements with a nearly uniform spatial light distribution in the focal plane, and in a bright sample to acquire the necessary statistics in a reasonable measurement time. We also had to assure a relatively uniform, stable local environment and avoid the fluorescence quenching often observed for densely labelled biological samples ${ }^{20}$ or fluorescent beads, as well as the heterogeneity of the single-molecule approach to photobleaching measurements ${ }^{21}$. Measurements with overlapped Gaussian excitation and de-excitation focal spots inside a non-concentrated dye solution fulfilled these requirements and allowed to obtain quantitative data for important parameters in STED microscopy, at the popular wavelength of $750 \mathrm{~nm}$ applied here: fluorophore de-excitation (i.e., reversible fluorescence silencing by excited-state depletion), irreversible photobleaching, and excitation by absorption of STED-light photons (resulting in STED-light-induced fluorescence) for various STED photon fluxes. Measurements were performed for several commercially available dyes using a fluorescence recovery approach ${ }^{22,23}$. We modified the pulse duration of STED by spectral phase shaping of the Ti:Sapphire laser emission by either a spatial light modulator (SLM) for the short pulse regime, or different lengths of optical fibers for the long pulse regime. In the first case, we were able to create arbitrarily shaped STED pulses of durations up to a few picoseconds (further increases of the pulse duration were limited by the maximal phase retardation of the SLM and the finite laser bandwidth). Using the second approach, we controlled the pulse durations up to $\sim 500 \mathrm{ps.}$ We compared results from experiments across this large range of pulse durations to CW and gated CW (gCW) STED operation ${ }^{24-26}$. Further, we examined the experimental data within a simple photobleaching model, which predicts the influence of STED photon distributions in imaging. The model allowed us to obtain information that is difficult to access experimentally, namely the resolution and spatially dependent probability of photobleaching with respect to the targeted coordinate. The goal was to identify optimal parameters for providing the STED de-excitation photons (in the time domain), previously studied theoretically ${ }^{27}$ without including photobleaching, to collect maximal information from the sample during imaging, while at the same time reducing undesirable interactions of fluorophores with the photons applied, conceptually, for de-excitation only. When working with the model to guide choices of future experimental parameters, we were interested in temporal photon distributions within the natural fluorescence decay of the fluorophores studied. CW de-excitation was not considered in our theoretical modelling. This modality, while it features technical simplicity and lower cost of implementation, has the drawback that a constant photon flux acts on the sample also at times when all the fluorophores are already in the dark "off"-state (no fluorescence is expected), which results in increased STED-light-induced fluorescence and bleaching during these times.

\section{Methods}

Experimental setup. The experimental setup is presented in Fig. 1a. Both the excitation and the STED pulse were created using a commercial mode-locked Ti:Sapphire laser (MaiTai HP, Spectra-Physics) operating at $750 \mathrm{~nm}$ wavelength with $80 \mathrm{MHz}$ repetition rate and initial pulse duration of $\sim 100 \mathrm{fs}(8.5 \mathrm{~nm}$ bandwidth). The excitation pulse (shown in blue), with duration of a few hundred femtoseconds, was generated in a supercontinuum fiber (SCG-800, Newport) and limited to $10 \mathrm{~nm}$ bandwidth at $635 \mathrm{~nm}$ central wavelength by a laser line clean-up filter BP (Z635/10X, Chroma). The STED pulse (shown in red) was temporally shaped in different ways, depending on the required pulse duration. For short pulses (0.1-3 ps) a home-built prism pre-compressor and pulse shaper were used. The compressor comprised two SF11 prisms which pre-compensate the later optical elements. The pulse shaper consisted of a diffraction grating DG (2400 lines/mm, PC 2400 NIR, Spectrogon), a long focal length lens $\mathrm{f}=400 \mathrm{~mm}$ and a liquid crystal spatial light modulator SLM (SLM-S640d, Jenoptik) in double-pass configuration. Temporal shaping was performed by applying different spectral phases to the de-excitation pulse. For long pulses ( $>10 \mathrm{ps}$ ), polarization-maintaining $(\mathrm{PM})$ fibers of different lengths were used (PM630-HP, Thorlabs; PMC-600-4, Schäfter\&Kirchhoff; PMJ-A3AHPC, OZ Optics). The relative time between excitation and STED pulses was adjusted by an optical delay line DL (Supplementary Information). 
a

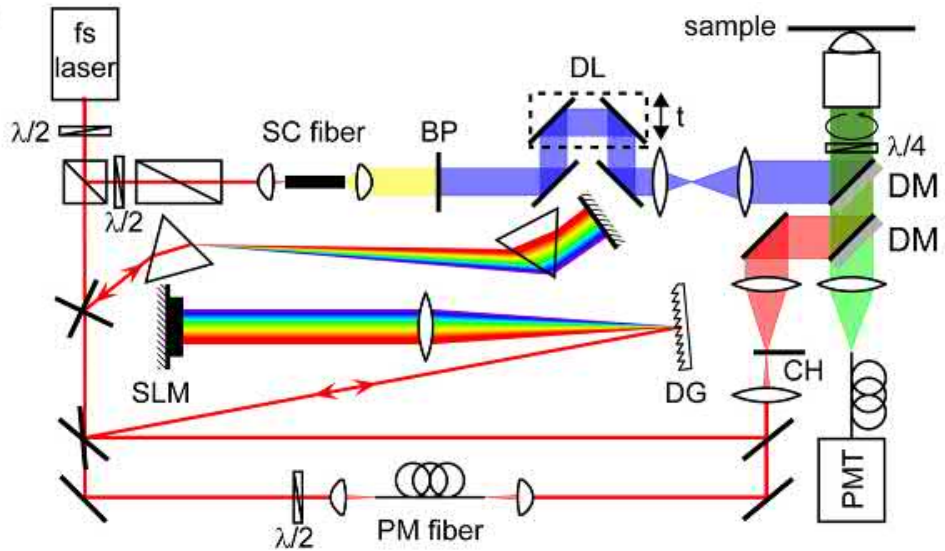

Exc

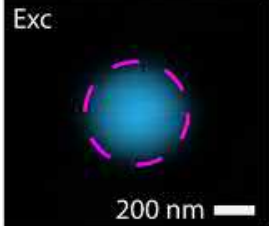

STED

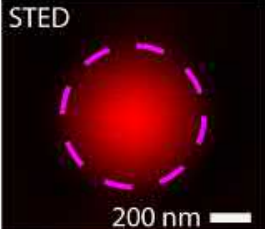

b

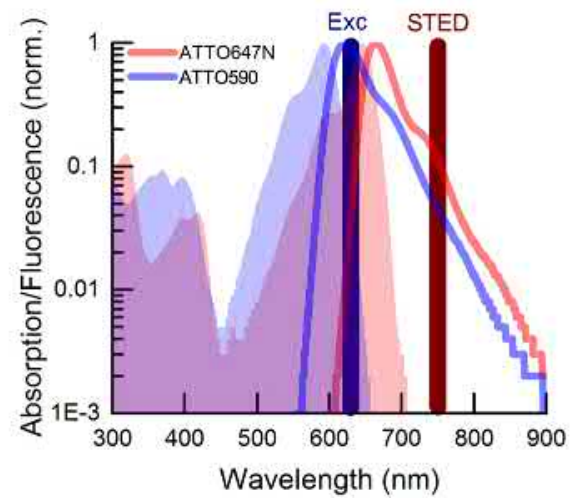

C

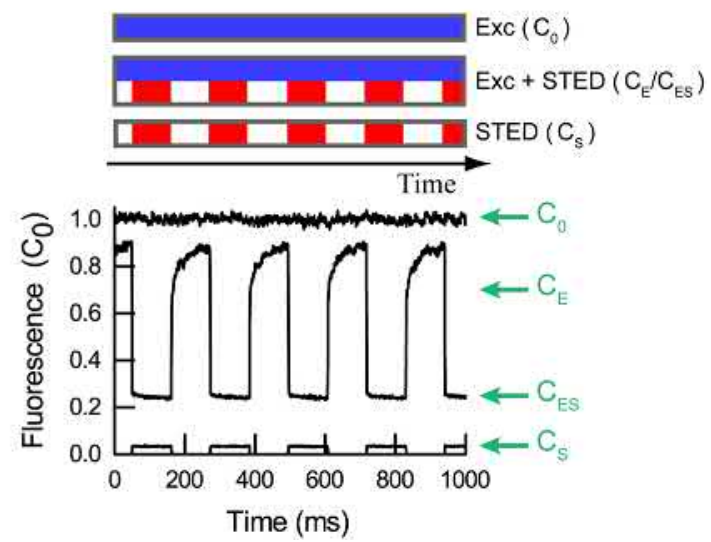

Figure 1. Experimental setup and measurement principle to extract STED de-excitation, bleaching and STEDlight-induced fluorescence. (a) Microscope with controllable fluxes of STED de-excitation photons. The laser was a femtosecond oscillator operating at $750 \mathrm{~nm}$. The excitation pulse was generated in a supercontinuum (SC) fiber. The excitation wavelength was spectrally selected by the bandpass filter BP $(635 \mathrm{~nm} / 10 \mathrm{~nm})$ and synchronized to the de-excitation pulse by the optical delay line DL. The STED pulse shape was controlled in two ways: for short pulses (0.1-3 ps), the optical path consisted of SF11 prisms as pre-compressor and a homebuilt pulse shaper. The pulse shaper was formed by the diffraction grating DG, the long focal-length lens and the spatial light modulator SLM placed in the Fourier plane created by those elements. For long pulses (10-500 ps, $\mathrm{CW}$ ), the optical path consisted of polarization-maintaining (PM) fibers of various lengths. CW operation was obtained by preventing mode-locking of the Ti:sapphire laser, and an additional diode laser was used as excitation source in this case (path not shown). To register fluorescence recovery curves, the STED beam intensity was chopped. The excitation (blue) and de-excitation (red) pulses were coupled into the microscope by dichroic mirrors DM and focused into the sample by an objective lens. The fluorescence (green) was collected in back-propagation and registered by a hybrid photomultiplier PMT in a confocal arrangement, with the data acquisition synchronized to the chopper wheel $(\mathrm{CH})$. The insets represent the focal spots of excitation (Exc) and STED beam, as measured by scattered signal of $80 \mathrm{~nm}$ gold beads. The dashed circles correspond to the FWHM of intensity. (b) Normalized spectral properties of Atto647N and Atto590, with the respective laser wavelengths for excitation and STED. The detection was centred at $690 \mathrm{~nm}$ and had $60 \mathrm{~nm}$ width (not shown). (c) Example excerpt of raw data set for a single measurement (Atto647N), with characteristic levels extracted to derive bleaching, de-excitation and STED-light-induced fluorescence. Each measurement consisted of three intensity traces (curves), registered sequentially: excitation-only, excitation with chopped STED and chopped STED only (acquisition time in each case: $\sim 100 \mathrm{~s}$ ). $\mathrm{C}_{0}, \mathrm{C}_{\mathrm{E}}, \mathrm{C}_{\mathrm{ES}}, \mathrm{C}_{\mathrm{S}}$ denote respectively: $\mathrm{C}_{0}$ the initial signal of fluorescence (due to the excitation beam), $\mathrm{C}_{\mathrm{E}}$ the fluorescence signal right after end of exposure to the STED beam, $\mathrm{C}_{\mathrm{ES}}$ the residual fluorescence signal in the presence of both the excitation and STED (de-excitation) beam, and $\mathrm{C}_{S}$ the fluorescence signal caused by the STED light. Signals correspond to respective fractions of fluorophores. Excitation: $\sim 500$ fs pulse duration (FWHM), $30 \mu \mathrm{W}$ average power; STED (shown measurement): $\sim 25$ ps pulse duration (FWHM), $50 \mathrm{~mW}$ average power. The delay between excitation pulse and STED pulse was $\sim 50 \mathrm{ps}$. All powers were measured at the back aperture of the objective lens. The powers in the focal plane were $\sim 70 \%$ of these values due to cut-off at the entrance pupil and transmission of the objective lens.

CW measurements were obtained by suppressing the mode-locking of the Ti:Sapphire laser and using an additional pulsed excitation laser at $80 \mathrm{MHz}$ (LDH-P-635, PicoQuant). All beams were expanded and coupled into the microscope using dichroic mirrors DM. The objective was an immersion oil lens of high numerical aperture 
(HCX PL APO $100 \times / 1.4$ NA, Leica). The excitation and STED focal spots were spatially overlapped, with the excitation focal spot nearly diffraction-limited and the STED spot enlarged $\sim 1.5$ times to ensure a more uniform illumination across the excitation focus. We realized confocal fluorescence detection, employing a fiber-coupled hybrid photomultiplier PMT (HPM-100-40, Becker\&Hickl) and bandpass filter (HQ690/60, AHF). The STED beam intensity was modulated by a fast chopper $\mathrm{CH}$ (3501 Optical Chopper, New Focus) with rise and fall times of $30 \mu \mathrm{s}$ at $4.5 \mathrm{~Hz}$ frequency, similarly to early experiments ${ }^{22,23}$. The dynamics of fluorescence recovery after stopping the STED beam by the optical chopper allowed us to disentangle the irreversible bleaching and reversible fluorescence inhibition by de-excitation (Fig. 1c). The fast recovery component corresponds to the fraction of signal instantaneously silenced by stimulated emission. The slow component is related to translational diffusion of fresh molecules into the focal spot and its magnitude represents the STED-light-induced photobleaching in the focal region (see further discussion in the Supplementary Information).

Photobleaching measurements. All measurements were carried out with a stationary focus (to avoid position drift of the scanning system, as often observed in long-term experiments with molecular layers) placed into dye solution samples to fulfill uniform chemical conditions. We chose to investigate four commercially available organic dyes in detail. These were: two standard red-emitting dyes for the STED laser wavelength applied in this work (ATTO647N, STAR635P), as well as two dyes with spectra shifted to the blue (ATTO590, STAR580). These dyes were obtained from ATTO-TEC (Siegen, Germany) and Abberior (Göttingen, Germany). The example spectral properties of both groups of dyes are presented in Fig. 1b. The fluorophores were dissolved in thiodiglycol $\left(2,2^{\prime}\right.$-thiodiethanol, TDE, $\mathrm{S}\left(\mathrm{CH}_{2} \mathrm{CH}_{2} \mathrm{OH}\right)_{2}$, Sigma) as obtained from the bottle. The solvent was chosen such that its viscosity allowed for slow re-diffusion of fresh dye molecules into the focal spot region, and thus slow fluorescence recovery to compensate for bleached dyes. Long exposure to the STED light $(\sim 100 \mathrm{~ms}$ at a time) ensured to include photobleaching mediated by the triplet state (triplet lifetime $\tau_{t r} \approx 1 \mu \mathrm{s}-10 \mathrm{~ms}$ ) or other long-lived dark states. All samples were enclosed between a concavity microscope slide and a standard cover glass sealed with nail polish. Each measurement was taken in steady-state conditions, with signals averaged over $\sim 100 \mathrm{~s}$. One such averaged data set consisted of three intensity traces, obtained separately (Fig. 1c): one for the excitation beam applied alone, one for excitation light together with the periodically blocked STED beam, and one for the modulated STED beam alone. All curves were normalized to the initial fluorescence signal registered for excitation light applied on its own $\left(C_{0}\right)$. From the characteristic levels $C_{E}, C_{E S}, C_{S}$, the STED de-excitation (depletion of excited state) $(D)$, bleaching $(B)$ and STED-light-induced fluorescence $(S F)$ can be derived as follows ${ }^{11}$ :

$$
\begin{gathered}
D=1-\frac{\left(C_{E S}-C_{S}\right)}{C_{E}} \\
B=1-C_{E} \\
S F=C_{S}
\end{gathered}
$$

The excitation power was kept constant $(30 \mu \mathrm{W})$ in the experiments described.

\section{Results}

STED de-excitation photon flux influence on "on"-"off" switching contrast and photobleaching. Temporal distribution of de-excitation photons: short de-excitation pulses (0.1-3 ps). To characterize photobleaching, we investigated the influence of the pulse duration $(\tau)$ for short de-excitation pulses $(0.13-3 \mathrm{ps})$, delayed slightly ( $7 \mathrm{ps})$ with respect to the ultrashort excitation pulse. All measurements were performed for the same dye concentration $(10 \mu \mathrm{M})$ and de-excitation pulse energy $\left(E_{p}=0.125 \mathrm{~nJ}\right.$, STED power $\mathrm{P}=10 \mathrm{~mW}$ ). Results for ATTO647N are presented in Fig. 2a. For de-excitation pulses comparable in duration to vibrational relaxation of the excited state, the de-excitation efficiency decreases due to unproductive de-excitation/excitation cycles related to the high STED photon flux, before vibrational relaxation can occur ${ }^{1}$. With increasing pulse durations, de-excitation is more efficient. Our data indicates that bleaching is a nonlinear process, as it depends on the pulse duration as $\left(E_{p} / \tau\right)^{b} \tau \sim \tau^{-(b-1)}$, where $b$ is the order of nonlinearity. The observed nonlinearity results from the interaction of excited molecules with photons that are applied to induce the de-excitation. Destructive chemical reactions often occur following optical transitions to higher (more reactive) excited states of the fluorophore. The fluorescence induced by the STED beam in this case (ATTO647N) is nearly negligible (typically $\sim 5 \%$ of initial excitation). The observation of increased STED-light-induced fluorescence for short pulses $(<1.5 \mathrm{ps})$ indicates both a reduction of excited-state depletion and higher involvement of two-photon absorption compared to the longer pulses.

The order of nonlinearity $b$ for bleaching and the order of nonlinearity $s f$ of STED-light-induced fluorescence with intensity can be retrieved as the line slopes in log-log representation (Fig. 2b,c). Analyzing the dependencies for different dyes gives respective values of the photobleaching order in the range $b \approx 1.4-1.9$ and STED-light-induced fluorescence $s f \approx 1.5-2.2$ (Table 1). For the red dyes ATTO647N and STAR635P, the SF signal was negligible $(\sim 5 \%)$. For this class of dyes, the major interaction between ground-state molecules and STED photons is by one-photon, Anti-Stokes linear absorption, which raises hot molecules to the first excited state. These results are in good agreement with the expected linear absorption cross-section at the used STED wavelength $\left(\sim 2 \cdot 10^{-5}\right.$ of the maximal excitation absorption cross-section for ATTO647N) and the low average STED power $(10 \mathrm{~mW})$ used in the experiments. Since unwanted excitation by the STED beam at $10 \mathrm{~mW}$ is negligible (a few percent of initial excitation), most photobleaching processes are initiated by the interaction of already 
a

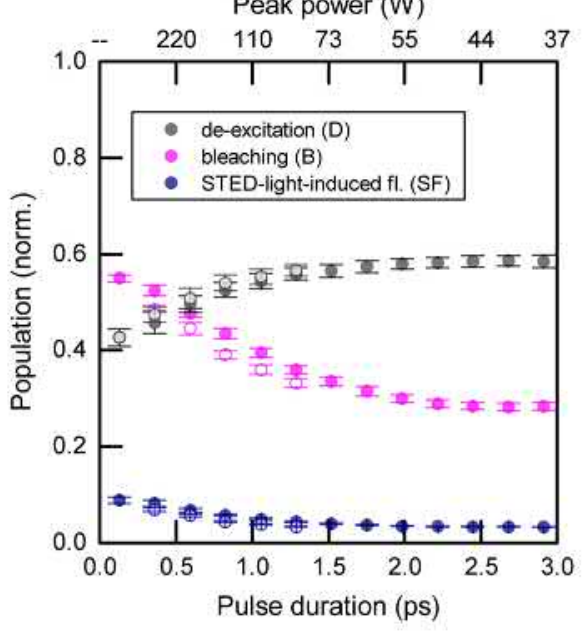

C

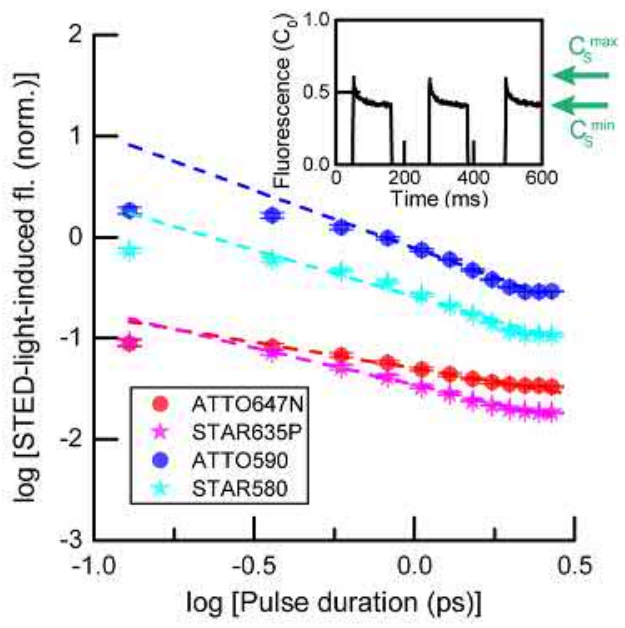

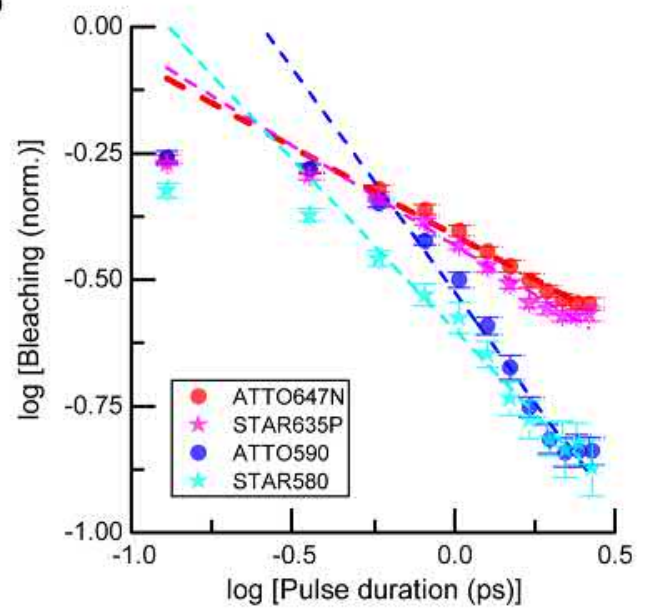

d

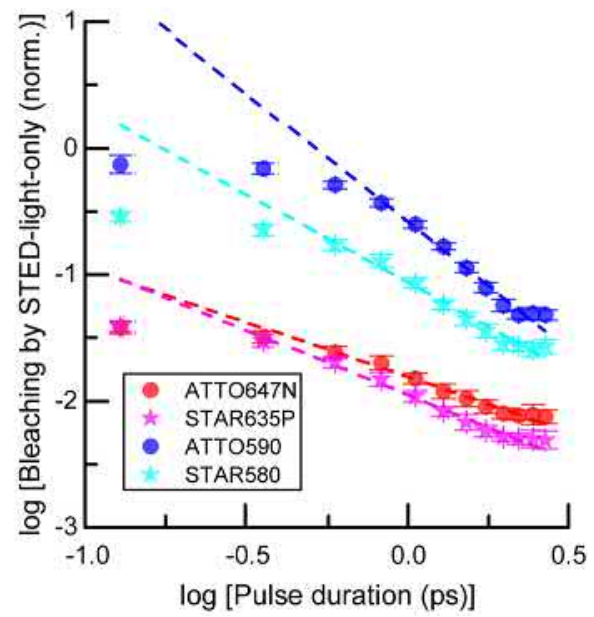

Figure 2. Pulse duration influence (0.1-3 ps) on de-excitation, bleaching and fluorescence induced by the STED beam at $750 \mathrm{~nm}$. (a) Example ATTO647N: De-excitation, bleaching and STED-light-induced fluorescence vs. STED pulse duration. Hollow data points represent down-chirped STED pulses. (b-d) Bleaching (b), STED-light-induced fluorescence (c) and bleaching induced by the STED light on its own (d) vs. STED pulse duration for four dyes: ATTO647N, ATTO590, STAR635P and STAR580. Data is shown in log-log representation. The magnitude of bleaching by the STED pulse alone (d) was calculated from the reduction in the STED-light-induced fluorescence signal ((c), inset (example for ATTO590) - see text). Time-averaged STED power: $10 \mathrm{~mW}$. All measurements were carried out for the same dye concentration $(10 \mu \mathrm{M})$.

\begin{tabular}{|l|l|l|l|}
\hline & $\begin{array}{l}\text { STED-light-induced } \\
\text { fluorescence }(\boldsymbol{s} f)\end{array}$ & Bleaching $(\boldsymbol{b})$ & $\begin{array}{l}\text { Bleaching by } \\
\text { STED only }\end{array}$ \\
\hline ATTO647N & $1.52 \pm 0.02$ & $1.39 \pm 0.02$ & $1.86 \pm 0.05$ \\
\hline ATTO590 & $2.15 \pm 0.07$ & $1.89 \pm 0.06$ & $3.02 \pm 0.08$ \\
\hline STAR635P & $1.73 \pm 0.02$ & $1.35 \pm 0.02$ & $2.03 \pm 0.04$ \\
\hline STAR580 & $1.92 \pm 0.05$ & $1.66 \pm 0.04$ & $2.40 \pm 0.07$ \\
\hline
\end{tabular}

Table 1. Orders of nonlinearity for STED-light-induced fluorescence, photobleaching and photobleaching by STED light on its own, as measured by varying the STED pulse duration over the range $0.1-3 \mathrm{ps}$. Tabulated coefficients are retrieved from line slopes presented in Fig. 2b-d, also shown in Fig. 3b,c. Time-averaged STED power: $10 \mathrm{~mW}\left(\mathrm{E}_{\mathrm{p}}=0.125 \mathrm{~nJ}\right)$.

excited molecules with STED photons which did not perform the de-excitation within the excitation focal spot. For both red dyes, the bleaching shows a nonlinear dependence, with order of nonlinearity $b \approx 1.4$. For the orange-emitting dyes ATTO590 and STAR580, the STED-light-induced fluorescence was comparable or higher 
a

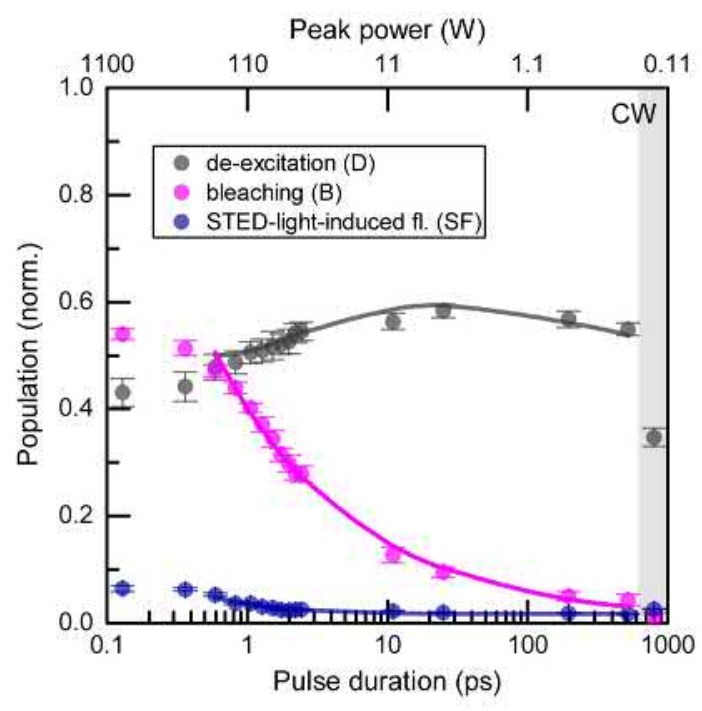

b

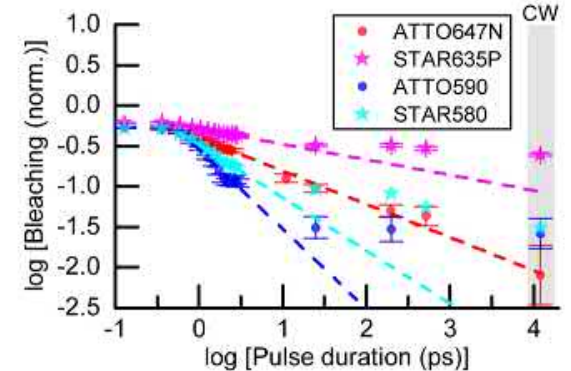

C

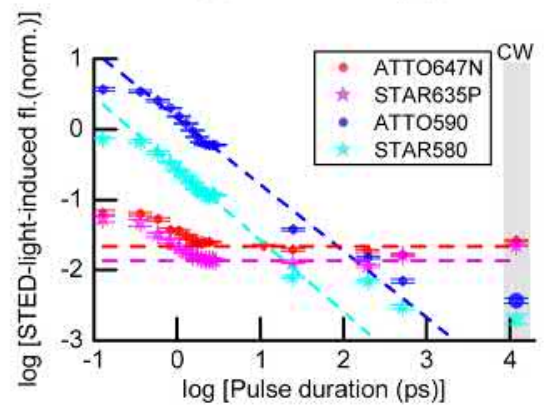

Figure 3. Pulse duration influence on de-excitation, bleaching and fluorescence induced by the STED beam at $750 \mathrm{~nm}$. (a) De-excitation, bleaching and STED-light-induced fluorescence vs. pulse duration for ATTO647N. CW represents measurements for continuous-wave STED laser operation. The solid lines represent values calculated within the photobleaching model (Supplementary Information). (b) Bleaching vs. STED pulse duration for different dyes. The dashed lines represent the orders of photobleaching, with values already determined for pulses of $0.1-3$ ps (Fig. 2). Deviations from this scaling indicate the dominant involvement of a different photobleaching pathway in these pulse-duration regimes (see text). (c) STED-light-induced fluorescence vs. STED pulse duration. For organic dyes typically used with the laser wavelengths applied in this study (ATTO647N, STAR635P), STED-light-induced fluorescence is mainly constant (flat dashed lines). For the orange-emitting dyes studied (ATTO590, STAR580), significant fluorescence emission induced by the STED beam occurs via two-photon absorption. Time-averaged STED power: $10 \mathrm{~mW}\left(\mathrm{E}_{\mathrm{p}}=0.125 \mathrm{~nJ}\right)$. Measurements were performed for a low dye concentration $(\sim 0.5-10 \mu \mathrm{M})$.

than the excitation signal, and a meaningful de-excitation efficiency cannot be specified (Fig. 2c). For these two dyes (ATTO590, STAR580), the mechanism of STED-light-induced fluorescence was two-photon absorption $(s f$ $\approx 2$, Table 1 ), manifesting as a strong increase $(\sim 1 / \tau)$ of the STED-light-induced fluorescence signal with shortening pulse duration (Fig. 2c). The bleaching showed a higher order of nonlinearity than for the red-emitting dyes, as it is initiated by multiphoton excitation by the STED beam at $750 \mathrm{~nm}$, which additionally populates the excited state. Moreover, for all four investigated dyes, the SF signal indicated strong bleaching due to the STED light alone acting on the molecules (defined as the difference in signal between maximal and minimal induced fluorescence $\left(C_{S}{ }^{\text {max }}-C_{S}{ }^{\text {min }}\right)$ during illumination by the STED light (Fig. $2 c$ inset and Fig. 2 d). Photobleaching due to the STED beam acting alone exhibited a higher-order dependence than the bleaching of already excited molecules (by the excitation light), for all four dyes examined (Fig. $2 \mathrm{~d}$ and Table 1). In this case, the effective order of photobleaching depends both on the order of the STED-light-mediated excitation mechanism (e.g. linear vs. two-photon) and on the order of the effective photobleaching rate starting from the first excited state with respect to intensity (related to the dominant photobleaching pathway, e.g. via the first triplet, or sequential absorption steps to higher states, see below). Interestingly, we observed a noticeable dependence of bleaching and STED-light-induced fluorescence on the chirp of the de-excitation light (Fig. 2a hollow points, see Supplementary Information). Chirped pulses are characterized by temporal delays of different spectral components along the ultrashort pulse. For all tested dyes and peak intensities, down-chirped STED pulses (led by the bluer components) showed $25 \%$ lower SF and $\sim 10 \%$ lower bleaching in comparison to up-chirped de-excitation pulses in this short-pulse regime. Within the measurement error, fluorescence de-excitation was similar in both cases. The error of measurement in the tabulated data represents the standard error of the line fit in the pulse duration range $>500 \mathrm{fs}$.

Longer de-excitation pulses ( $>10 \mathrm{ps}$ ). We extended our investigations to longer durations of de-excitation pulses (Fig. 3). Here, the delays between excitation and de-excitation pulses were adjusted to approximately the STED pulse duration, $\sim \tau$ (FWHM), to maintain the highest possible de-excitation (see Supplementary Information). The STED pulse energy was fixed during all measurements as before $\left(E_{p}=0.125 \mathrm{~nJ}\right)$, concentrations of the dye solutions were in the range $\sim 0.5-10 \mu \mathrm{M}$. While de-excitation and STED-light-induced fluorescence were nearly constant for ATTO647N over a large range of pulse durations (10-500 ps) (Fig. 3a), the non-recovered fraction of signal after STED-beam exposure representing bleaching, $B$, showed a strong dependence on pulse duration (Fig. 3a,b), being reduced from $B \approx 0.5$ for $\sim 1$ ps pulses to $B \approx 0.01$ or below for CW de-excitation. The least bleaching was observed for $\mathrm{CW}$, which also produced the lowest de-excitation: under 
CW conditions only a fraction ( $30 \%)$ of the STED-light photons act on the excited-state molecules and can potentially switch off fluorescence at the $80 \mathrm{MHz}$ repetition rate of excitation pulses, acting within the decay of spontaneous emission ( 3.5 ns). As for the example ATTO647N (Fig. 3a), the de-excitation efficiency was nearly constant over a broad range of pulse durations also for the other investigated dyes (not shown). For the orange-emitting dyes (ATTO590, STAR580), the STED-light-induced fluorescence showed a clear signature of two-photon absorption from STED pulses over the entire range of pulse durations, while both red-emitting dyes (ATTO647N, STAR635P) showed STED-light-induced fluorescence at a largely constant level for long pulses (25 ps to CW) (Fig. 3c), indicating linear absorption as the dominant STED-light-induced excitation mechanism.

Despite these differences in interaction between ground-state molecules with STED-light photons, photobleaching clearly depends on the peak intensity for all dyes, and the peak intensity should be as low as possible to reduce high-order photobleaching (Fig. 3b), while also seeking to maintain sufficient de-excitation. Notably, for ATTO647N, the bleaching followed a constant scaling law of order $\sim 1.4$ over the whole range of pulse durations (Fig. 3b). However, for the three other dyes examined, the bleaching showed different dependencies than expected based on the previous measurements in the short-pulse regime (Fig. 3b, dashed lines and deviations therefrom). This suggests that different photobleaching mechanisms ${ }^{28,29}$ (low vs. high order) play a limiting role at different (regimes of) peak powers, with different contributions of multi-step absorption processes of STED light vs. the constant bleaching (e.g. intensity-independent bleaching mediated by the build-up of the first triplet state or other dark states).

While there were differences in the absolute magnitudes of photobleaching in comparison to the data presented above, the order of photobleaching $b$ was found to be similar in both measurement series (compare Figs $2 \mathrm{~b}$ and $3 \mathrm{~b}$ ). The offset between them can be explained by different low-order photobleaching (e.g., photobleaching rate independent of intensity, related, for example, to the triplet state $T_{1}$ ). The difference likely arose due to a change of the solvent (TDE had to be taken from a different bottle for the experiments of Fig. 2 vs. those of Fig. 3 , with possibly slightly different oxygen content). We observed no difference in the photobleaching order with dye concentration changes $(1-10 \mu \mathrm{M})$ between the measurement series.

Power dependencies. The measurements described above were performed for much lower average power $(10 \mathrm{~mW})$ than typically applied in standard STED operation for high resolution levels $(>100 \mathrm{~mW})$ at this repetition rate. The reason is that we desired to perform the comparative study for the whole range of pulse durations. Yet, at just slightly higher average powers $(\sim 13 \mathrm{~mW})$, we had observed a drastic increase in STED-light-induced fluorescence (likely as a result of photolysis) for the shortest, $\sim 130 \mathrm{fs}$, STED pulses with peak power $\sim 1.2 \mathrm{~kW}$ (Fig. 4a, ATTO647N), a pulse length regime we desired to include in the series. To explore bleaching under more realistic STED conditions we further examined the influence of the STED photon flux by varying the average power for a set of different pulse durations (Fig. 4). Increasing the pulse duration from $130 \mathrm{fs}$ to $200 \mathrm{ps}$ or CW allowed to apply more de-excitation photons without increasing bleaching (Fig. 4b,c). Regardless of the nonlinear nature of photobleaching shown above for measurements with varied pulse duration (b $>1$, Figs 2 and 3 ), measurements as a function of STED power $P_{\text {STED }}$ revealed a sublinear bleaching dependency $\sim P^{b}$ with $b<1$ (Fig. 4a-c,e; Table 2), even for de-excitation pulses as short as $130 \mathrm{fs}$. As previously, we determined the orders of nonlinearity $b$ and $s f$ for long pulse durations $(\sim 25 \mathrm{ps}, \sim 200 \mathrm{ps}, \sim 500 \mathrm{ps})$ by line fits in log-log representation for all investigated dyes (Fig. $4 \mathrm{~d}, \mathrm{e}$ ). Surprisingly, $b$ and $s f$ did not vary significantly over the investigated range of pulse durations. Averaged values for all dyes are presented in Table 2, with errors of measurement estimated from maximal and minimal deviations from the line fit (Fig. 4d,e). The sublinear power dependence of photobleaching is related to the drastic decrease in occupation of the excited state with increasing STED power (pulse energy), which masks the underlying mechanism(s) of photobleaching as a function of photon flux (instantaneous intensity).

We observed similar photobleaching behaviours for 200 ps Gaussian pulses and for CW, however, to maintain the same de-excitation efficiency, the STED average power has to be $\sim$ five-fold higher in the second case. This mandatory power increase results in increased STED-light-induced fluorescence for the CW case, due to increased linea absorption of STED photons. Similar de-excitation to $200 \mathrm{ps}$ STED pulses, at the same power level, can be obtained by applying gated detection $(\mathrm{gCW})^{25,26}$, at the expense of detected fluorescence signal (Fig. $4 \mathrm{c}$, hollow points). For the gCW measurements, we registered the signal starting at $\sim \tau_{f l} / 2$ after excitation, where $\tau_{f l}=3.5 \mathrm{~ns}$ denotes the fluorescence lifetime. This meant that the fluorescence signal was reduced by $\sim 40 \%$ by gating. Note that, for gCW, the STED-light-induced fluorescence signal, as detected with gating, is higher than for CW STED at the same power. The determined bleaching coincided for both cases. Maintaining the same signal for dim samples requires longer dwell times and would increase photobleaching due to increased exposure times.

Influence of STED photon flux in imaging. We modelled the influence of the STED photons on the resulting super-resolution image by simplifying the involved molecular electronic states to a standard 4-level system (Fig. 5a and Supplementary Information). We chose this system as it described the de-excitation efficiency over the whole investigated temporal range, including population-inversion for short pulses (see reduction in the de-excitation $D$ for $\tau<5$ ps in Fig. 3a). To include photobleaching, we inserted an additional state $(\beta)$ corresponding to irreversibly bleached molecules. We assumed that photodestruction occurs by interaction of excited molecules $\left(\mathrm{S}_{1} *, \mathrm{~S}_{1}\right.$ states $)$ with the STED photons, i.e., molecules in the ground state $\left(\mathrm{S}_{0} *, \mathrm{~S}_{0}\right)$ are chemically stable, and the bleaching driven by the excitation light is negligible. Initially, all molecules are in the ground state and can be transferred to the $\mathrm{S}_{1}$ * state by linear absorption of photons (from the excitation or STED pulse) or by two-photon absorption of STED photons. Then, after vibrational relaxation to $S_{1}$, molecules can relax to $S_{0} *$ by emitting fluorescence, or be transferred to the state $\mathrm{S}_{0} *$ by stimulated emission, or photobleach to end up in $\beta$. The paths taken by the excited-state fluorophore depend on the instantaneous STED intensity and probabilities of each process, which have to be found experimentally. We chose to model the behaviour of ATTO647N, as the 
a

Peak power $(\mathrm{W})$

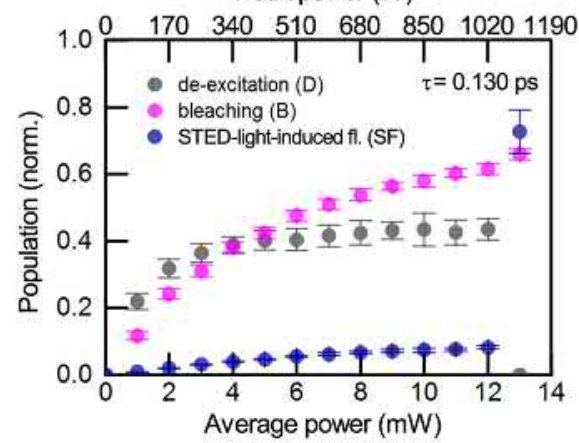

d
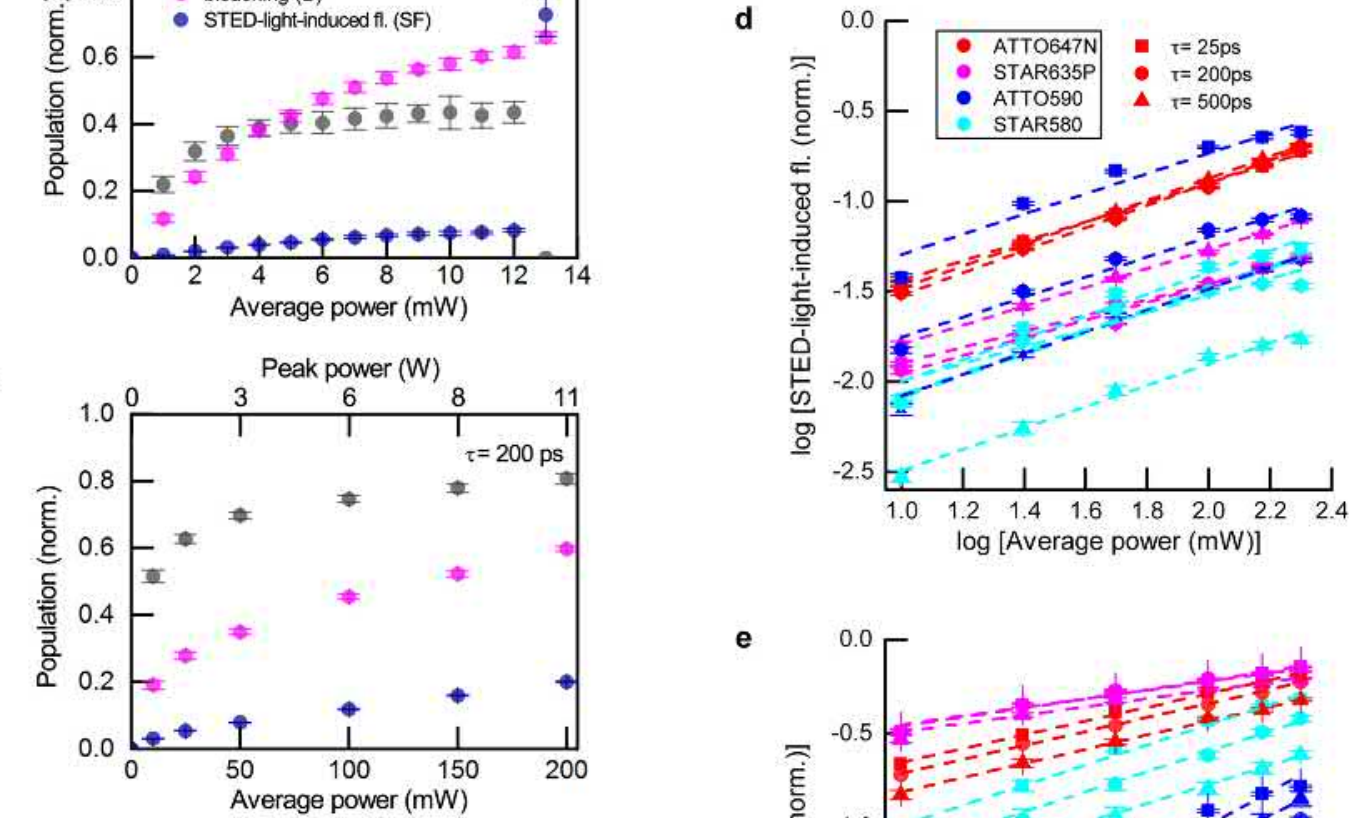

c

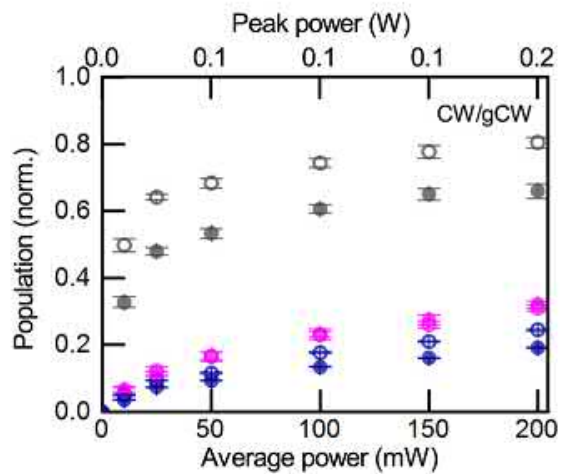

e

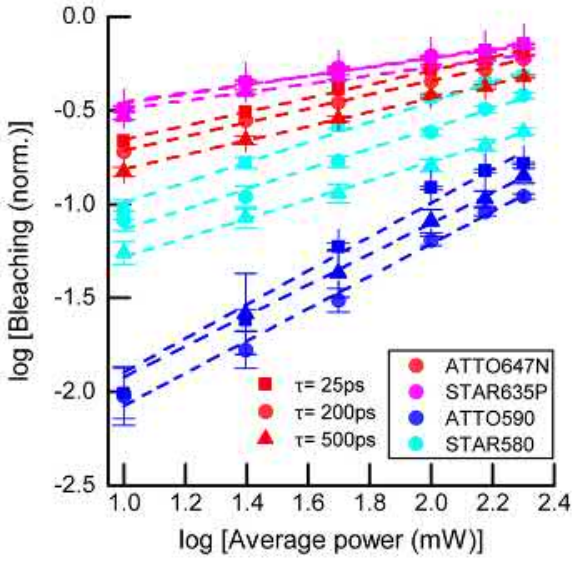

Figure 4. Influence of STED power on de-excitation, bleaching and fluorescence induced by the STED beam. (a-c) De-excitation, bleaching and STED-light-induced fluorescence vs. time-averaged power for Gaussian STED pulses of $130 \mathrm{fs}(\mathbf{a}), 200 \mathrm{ps}(\mathbf{b})$ duration, and for CW STED (c). The hollow circles in (c) indicate CW STED operation with time-gating of detected fluorescence (see text). Measurements in (a-c): ATTO647N. (d) STED-light-induced fluorescence vs. power for different dyes and different pulse durations. (e) Bleaching vs. power for different dyes and different pulse durations. Note the log-log representation of data in $(\mathrm{d}, \mathrm{e})$.

\begin{tabular}{|l|l|l|}
\hline & $\begin{array}{l}\text { STED-light-induced } \\
\text { fluorescence } \mathbf{( s f})\end{array}$ & Bleaching $(\mathbf{b})$ \\
\hline ATTO647N & $0.59 \pm 0.04$ & $0.37 \pm 0.01$ \\
\hline ATTO590 & $0.54 \pm 0.06$ & $0.9 \pm 0.1$ \\
\hline STAR635P & $0.49 \pm 0.04$ & $0.23 \pm 0.01$ \\
\hline STAR580 & $0.59 \pm 0.04$ & $0.53 \pm 0.03$ \\
\hline
\end{tabular}

Table 2. Orders of nonlinearity of bleaching and STED-light-induced fluorescence, as measured by varying the STED average power. Tabulated coefficients are retrieved from line slopes of the lines presented in Fig. 4d,e. 
a

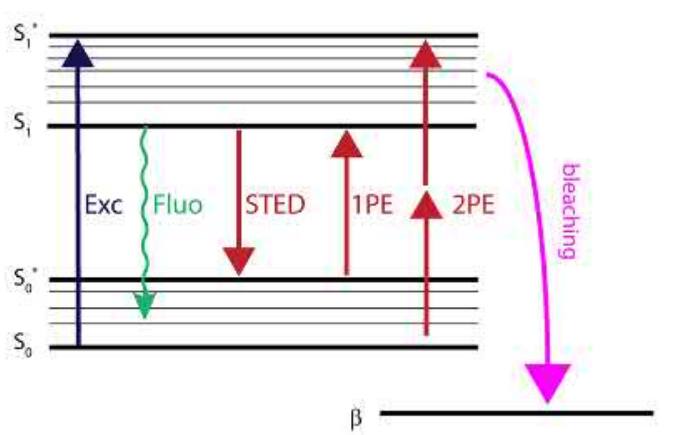

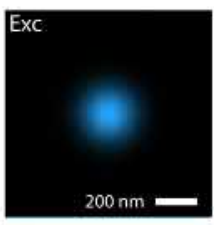

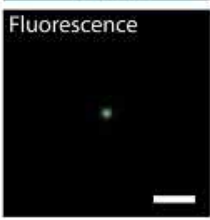

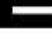

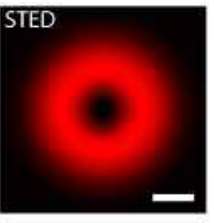

Bleaching

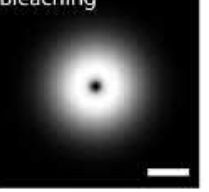

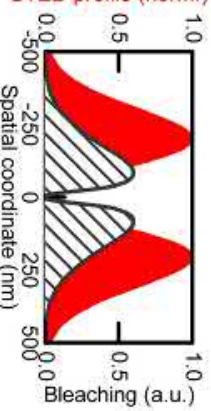

d

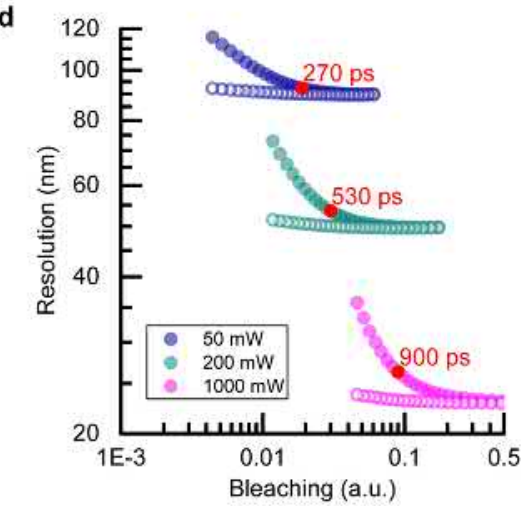

e
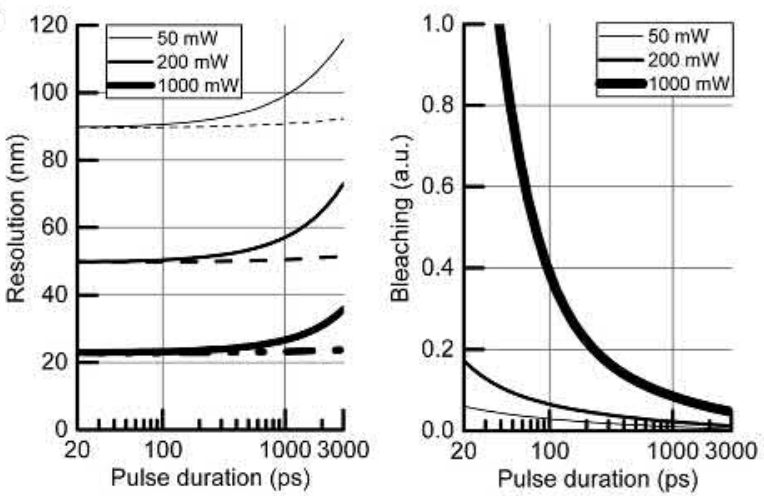

f
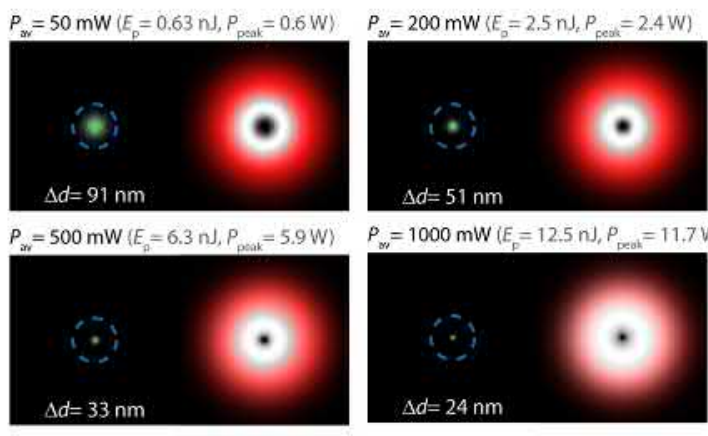

$P=1000 \mathrm{~mW}(E=12.5 \mathrm{~nJ}, P=11.7 \mathrm{~W}$

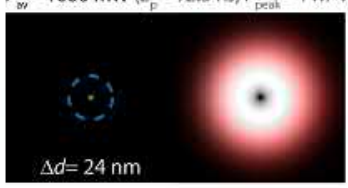

Figure 5. Simulation of the STED performance at $750 \mathrm{~nm}$ in imaging (based on parameters derived from measurements with ATTO647N). (a) Simplified model of de-excitation, bleaching and STED-light-mediated 1-photon (1PE) and 2-photon (2PE) excitation processes. (b) Focal-plane excitation (Exc) and STED spatial profiles (top). Resulting fluorescence and probability of photobleaching (bottom), shown here for a STED pulse duration of $\tau=200 \mathrm{ps}$ and average power $\mathrm{P}=450 \mathrm{~mW}$ ( $80 \mathrm{MHz}$ repetition rate). (c) Resolution and bleaching as a function of pulse duration for three time-averaged STED powers. The dashed lines represent gated detection. (d) The same data as in c presented as resolution vs. bleaching. The lowest bleaching and the highest resolution can be obtained by adjusting the STED pulse duration and applying gated detection (hollow points, $\mathrm{t}_{\text {gate }}=\tau$ ). (e) Influence of residual STED intensity $\zeta$ at the targeted coordinate (intensity minimum) on resolution and fluorescence signal amplitude for three different levels: 0, 0.0025 and 0.01 of doughnut crest intensity. STED pulse duration: $\tau=1000 \mathrm{ps}$, gated detection $\mathrm{t}_{\text {gate }}=\tau .1000 \mathrm{~mW}$ at $80 \mathrm{MHz}$ repetition rate (this simulation) correspond in terms of pulse energy and peak power to $250 \mathrm{~mW}$ at $20 \mathrm{MHz}$, a common repetition rate for STED with nanosecond pulses. Note that the three black curves (resolution) overlap. (f) Spatial distribution of fluorescence (green), normalized probabilities of photobleaching (grey scale, with highest photobleaching in white) and normalized STED beam profile as reference (red) for different average STED powers (STED pulse duration: $\tau=1000 \mathrm{ps}$ ). The stated resolution values $\Delta \mathrm{d}$ represent the respective FWHM of the fluorescence profile.

data for this dye suggested a single scaling of photobleaching over the whole range of pulse lengths (peak powers), as $\sim I_{S T E D}{ }^{1.4}$ (Fig. 3b). To find the optimal parameters which simultaneously describe the three experimental curves $(D, B, S F$ in Fig. $3 b)$ in a global fit analysis, we varied the STED pulse durations to match measurements and numerically calculated the expected values for de-excitation, bleaching and STED-light-induced fluorescence in our confocal detection scheme, for 3D spatial distributions of excitation and STED foci (Supplementary Information). We inferred the following parameters: stimulated emission cross-section $\sigma_{S T E D}=4.8 \cdot 10^{-18} \mathrm{~cm}^{2}$, 
cross-section for linear excitation by the STED beam $\sigma_{1 P E}=3 \cdot 5 \cdot 10^{-21} \mathrm{~cm}^{2}$, cross-section for two-photon excitation by the STED beam $\sigma_{2 P E}=3.5 \cdot 10^{-50} \mathrm{~cm}^{4}=3.5 \mathrm{GM}$, and an effective photobleaching coefficient from the excited state $k_{1}=5.2 \cdot 10^{-10} \mathrm{~Hz}$. The intensity-dependent photobleaching rate $k$ was defined as $k=k_{1} \cdot\left(I / I_{0}\right)^{b}$, with $b=1.4$ and $I_{0}=1 \mathrm{~W} / \mathrm{m}^{2}$. The quality of the fit with respect to experimental data is seen in Fig. 3a (solid lines).

By introducing the spatial intensity distribution for the Gaussian excitation and doughnut-shaped STED profile in the focal plane, the effective fluorescence point spread function (PSF) and the spatially dependent probability of photobleaching were numerically calculated in the focal-plane, based on the inferred parameters (Fig. 5b,f). We defined the resolution as the full width at half maximum (FWHM) of the fluorescence profile, and computed photobleaching as proportional to the integral over its spatial distribution (Supplementary Information). To enable a fair comparison between different pulse durations, we chose the most favourable time delay between excitation and STED pulses, which was calculated numerically to be within FWHM/2 and FWHM of the Gaussian STED pulse duration for pulses longer than $100 \mathrm{ps}$ (Supplementary Information).

We modelled the resolution and photobleaching as a function of Gaussian pulse duration for different time-averaged STED powers, with results for $50 \mathrm{~mW}, 200 \mathrm{~mW}$ and $1 \mathrm{~W}$ shown in Fig. $5 \mathrm{c}$. Differences in de-excitation pulse duration affect both the de-excitation efficiency and the bleaching, and we thus additionally present resolution as a function of photobleaching (rather than separate duration dependencies) to facilitate comparisons (Fig. 5d). The resolution, similarly to de-excitation (its enabling process), is independent of pulse duration over a broad range of pulse durations (20-200 ps), as stimulated emission is a linear process in pulse energy, i.e. linear with the number of photons applied. In contrast, the bleaching shows a strong dependence on pulse duration. For example, by changing the duration from 200 ps to $1000 \mathrm{ps}$ at $200 \mathrm{~mW}$ average STED power, resolution drops by only $\sim 10 \%$, with a reduction of bleaching by more than $50 \%$. Optimal pulse duration (the best compromise between resolution and bleaching) depends on the available average power (approximately, the red points in Fig. 5d). Under low average power conditions (with low intensity-dependent bleaching, resolution $\sim 70 \mathrm{~nm}$ ), short pulses are preferable $(\sim 270 \mathrm{ps})$, as they provide the most efficient de-excitation from the excited state in non-gated STED operation. Higher-power applications are limited by photobleaching, and long de-excitation pulses ( ns) provide a better trade-off. Applying long pulses combined with gated detection (Fig. 5c, dashed lines; Fig. 5d, hollow circles) allows always to maintain the highest resolution and low bleaching, at the expense of a reduction of registered fluorescence signal. It is important to note that high STED powers require particularly low residual intensity at the coordinate targeted by the intensity minimum (Fig. 5e). For example, for a relative residual intensity of $\zeta \approx 1 \%$ at the intensity minimum (compared to the doughnut crest) and resolution level of $\sim 20 \mathrm{~nm}$, nearly $70 \%$ of the initial fluorescence amplitude is lost due to de-excitation of fluorophores directly at the doughnut minimum ${ }^{30}$.

Our model quite successfully described de-excitation, photobleaching and STED-light-induced fluorescence as a function of STED pulse duration (Fig. 3a). Nonetheless, applying the same model to measurements as a function of the average STED power led to inconsistencies (Fig. 6a): the measured de-excitation $D$ saturated at lower values and the measured STED-light-induced fluorescence $S F$ was significantly higher than expected from the model based on the previously determined parameters for ATTO647N. Nonetheless, for all measurements, photobleaching shows the same 'slope' as a function of STED power and is described by the proposed model (showing the same scaling with STED power). As mentioned above, for different ATTO647N samples with solvent from either bottle, the registered photobleaching shows different constant offset (low-order photobleaching, compare Figs $4 \mathrm{~b}$ and $6 \mathrm{a}$ squares), which was corrected in Fig. 6 by subtracting a constant value $(\sim 0.2)$ from one experimental data set (Fig. 6a squares).

The observed differences for de-excitation and STED-light-induced fluorescence may stem from the fact that other, additional electronic states are involved (Fig. 6b), which become relevant at higher STED powers. It is important to consider at least three other states: the higher excited state $S_{n}$, and the lower and higher triplet states $\mathrm{T}_{1}, \mathrm{~T}_{\mathrm{n}}^{31,32}$. The effects of bleaching from singlet states $\mathrm{S}_{\mathrm{n}}$ could be subsumed to processes from $\mathrm{S}_{1}$ (due to very fast relaxation times ( $\sim$ few ps) by internal conversion IC). However, it is challenging to include the involvement of triplet states in numerical simulations, due to their much longer time scale and ambiguities that arise if several new states, and therefore new parameters, are postulated. The observed increased STED-light-induced fluorescence at high average powers might be explained by delayed fluorescence: molecules trapped in the lower triplet state $T_{1}$ can be directly excited by STED light to higher triplet states $T_{n}$ and then, with some probability (defined by intersystem crossing ICS) come back to the electronic singlet system. The time scale of this process is related to the lifetimes of higher triplet states; for instance, the typical lifetime of the second triplet state $\mathrm{T}_{2}$ is $\sim 100 \mathrm{~ns}$. A five-level system, incorporating states as shown in Fig. $6 c$, is sufficient to describe photobleaching data in micros$\mathrm{copy}^{31,32}$. Nevertheless, the number of free parameters makes it less intuitive. Moreover, the influence of the photobleaching from the triplet or other dark states should be significantly reduced by application of low-repetition rate lasers and triplet quenchers, in which case the contribution of high-order photobleaching would become dominant. Note that the influence of the triplet system could in principle be elucidated by time-reversal of the excitation and STED pulse, respectively, and this was studied in prior work ${ }^{11}$. By applying repetition rates below $\mathrm{MHz}$, the involvement of all triplets can be reduced ${ }^{12,13}$ at the expense of significantly increased measurement time to collect fluorescence signal. Reduction of the repetition rate from $80 \mathrm{MHz}$ to e.g. $10 \mathrm{MHz}$ should reduce the build-up of the second triplet state (with lifetime $\sim 100 \mathrm{~ns}$ ) and reduce photobleaching associated with this state. The same outcome can be obtained by very fast scanning to allow dark-state relaxation ${ }^{5,14}$.

It is important to note that higher resolution demands higher pulse energies (sufficiently large numbers of de-excitation photons), and careful attention is required in choosing the fluorophore. For standard dyes such as ATTO647N, the linear absorption cross-section of the STED light is five orders of magnitude lower than the absorption cross-section at the excitation wavelength (Supplementary Information). This means that applying an excitation power of $\sim 3 \mu \mathrm{W}$ and a STED power of $\sim 300 \mathrm{~mW}$ will result in comparable numbers of molecules excited per unit volume by both beams. The difference is that the STED light also serves to de-excite molecules 

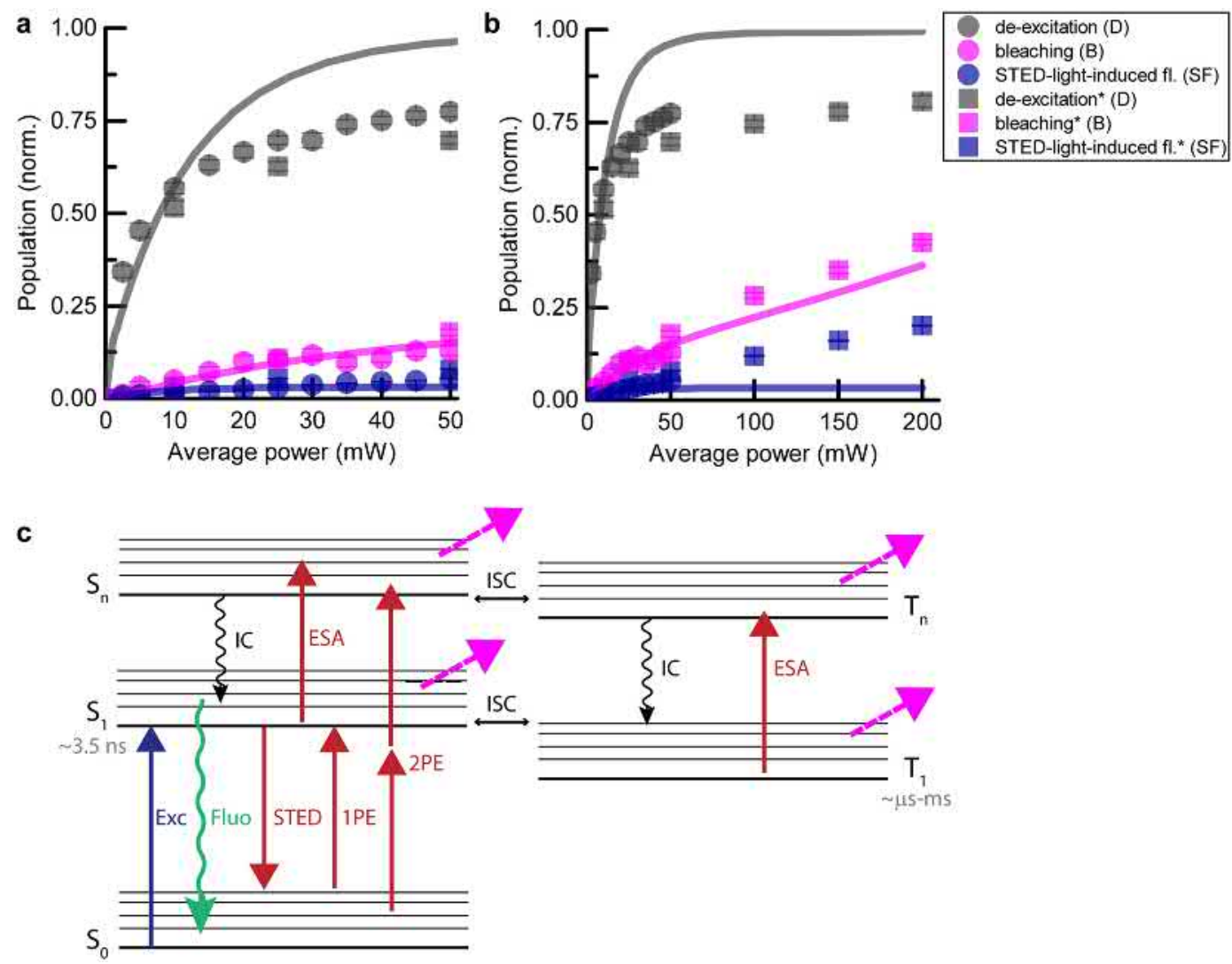

Figure 6. Limitations of the photobleaching model in explaining the data at high STED powers (ATTO647N) (see text). (a,b) Comparison between experimental and modelled data for de-excitation, bleaching and STEDlight-induced fluorescence vs. STED power. (a) represents a subset of the data shown in (b). Circles and squares correspond to two independent measurement series). (c) Extended model of states possibly involved in photobleaching ${ }^{32}$, including an additional excited singlet state $S_{n}$, and two triplet states $T_{1}$ and $T_{n}$. ISC: intersystem crossing; IC: internal conversion; ESA: excited-state absorption processes.

- meaning that those excitation events are followed by de-excitation and thus do not contribute to the measured signal. Additionally, confocal detection rejects signal from outside the excitation-light spot. The underlying interaction (STED light with molecule), however, causes major photobleaching in the peripheral region surrounding the targeted coordinate, i.e. predominantly at the doughnut crest (Fig. 5b, Supplementary Information) for high STED average powers. Shifting the STED wavelength to even longer wavelengths and/or using dyes with spectra shifted to shorter wavelengths should thus be beneficial for STED implementations with very high average powers where linear absorption of STED-light photons initiates further excitation steps and thus photobleaching. To illustrate the behaviour of a different (orange) dye, modelling for ATTO590 is discussed in the Supplementary Information (see Supplementary Fig. S8 and Supplementary Table S4). Careful studies are still needed to establish the spectroscopic properties of other states, and in particular to shed more light on the process of excited state absorption $^{33}$ (ESA) and its involvement at the STED wavelength for the dyes in use.

\section{Discussion}

In STED nanoscopy, efficiently de-exciting fluorophores outside the targeted coordinate is the key to super-resolution. High resolution requires to deliver the necessary numbers of photons while fluorophores reside in the excited state. Sufficient intensity close to the targeted coordinate is accompanied by much higher excess exposure at the doughnut crest. It is especially these high de-excitation light intensities which lead to unwanted photon absorption events and photobleaching. Depending on the photon flux acting on the excited fluorophores, two different photobleaching regimes can generally be distinguished for constant pulse energy: bleaching that is independent of the intensity (low-order photobleaching), and intensity-dependent bleaching which is associated with both singlet and triplet higher excited states (high-order photobleaching). One low-order photobleaching pathway is by reactions undergone by fluorophores trapped in the first triplet state, or other dark states. High-order bleaching occurs because of the more reactive nature of the higher excited states, which are populated by multiphoton or multiple sequential absorption steps.

In this study, we provide quantification of a number of experimental aspects in STED microscopy. Our data corroborate previous strategies that had been followed based on physical intuition, particularly the realization that longer pulses have advantages in minimizing photobleaching. The results highlight subtle but important differences 
even for dyes that have very similar emission spectra (STAR635P vs. ATTO647N), suggesting that different optical strategies, tailored to the particular dye and its environment during imaging, will best mitigate bleaching.

At low STED powers (e.g. $\sim 10 \mathrm{~mW}$ for ATTO647N), the de-excitation photons predominantly interact with fluorophores to induce stimulated emission, which serves to transfer them to the non-reactive ground state and thus reduce photobleaching in comparison to the excitation light acting alone ${ }^{14,34}$. As the STED intensity is increased for higher resolution, other interactions with the STED light cannot be neglected any more: absorption of STED-light photons, which raise the fluorophores to either the first excited singlet and/or higher excited singlet and triplet states, becomes more probable. Occupancy of each of these excited states contributes to the overall magnitude of bleaching. We argue that the exact chemical mechanisms need not strictly be known in detail to identify optimal parameters for STED imaging (pulse energy, pulse duration, repetition rate). Rather, the overall dependence of photobleaching on STED photon flux needs to be characterized, and this was the approach taken in this study. In this work, we verified the reduction of high-order photobleaching by lowering the peak intensity (using longer pulses), whereas low-order photobleaching is not strongly affected by pulse duration, and other strategies such as the aforementioned dark-state relaxation schemes ${ }^{13,14}$ and triplet quenchers hold more promise to reduce this contribution.

In our study, photobleaching measurements as a function of STED intensity, by varying the time-averaged power (Fig. 4), resulted in sub-linear dependence (orders $<1$ ), but measurements in which the pulse duration was varied (Figs 2 and 3) showed a super-linear dependence (orders $>1$ ). This can be explained as in measurements vs. power the numbers of delivered photons are varied, affecting both the de-excitation and bleaching in ways that are difficult to disambiguate. In measurements vs. pulse duration, the energy per pulse was constant (i.e., a fixed number of de-excitation photons), with little effect on the de-excitation (Fig. 3a), meaning that these measurements isolate the photobleaching behaviour, as they are not compromised by altered occupancies of the excited state.

A significant reduction of photobleaching was observed for increasing de-excitation pulse durations, which implies a high-order photobleaching mechanism (orders in the range 1.4 to 1.9) for all four investigated dyes at high peak powers of $10 \mathrm{~W}$ and above. Our data therefore reveal the involvement of high-order photobleaching at the currently applied STED peak powers ${ }^{2,8}$. For very short pulses $(0.13-3 \mathrm{ps})$, photobleaching by the STED beam action alone was noticeable. Moreover, for de-excitation pulses shorter than $\sim 2 \mathrm{ps}$, it was impossible to obtain efficient de-excitation due to numerous unproductive de-excitation/excitation cycles caused by the very high photon flux, as expected theoretically ${ }^{1}$ and seen in early experiments ${ }^{22}$. The number of these cycles was reduced to some extent by applying pulses with a down-chirp. The down-chirp not only reduced the STED-light-induced fluorescence but also the photobleaching (compared to the standard up-chirped pulses): effectively, fluorophores will be found in the reactive excited state for a shorter time (Supplementary Information). The influence of chirp for long de-excitation pulses is likely minor, as it depends on the vibrational lifetime ( $\sim 1 \mathrm{ps})$ and may only become relevant at high photon fluxes.

For lower photon fluxes (peak powers $<10 \mathrm{~W}$ ), three of the four dyes exhibited almost intensity-independent photobleaching for constant pulse energy. We found that ATTO647N followed a single scaling of bleaching for all peak powers (order 1.4), which may be explained by low ISC and thus negligible bleaching from the triplet system, in line with previous observations. The bleaching from the whole singlet system effectively scales like bleaching from the first singlet state, as the higher singlet states' lifetimes are shorter by orders of magnitude, and their bleaching contributions can be subsumed into bleaching from the first excited singlet state (with one effective rate, dependent on intensity due to involvement of the higher states).

Our results highlight the importance of the STED-light absorption. It leads to a small contribution to fluorescence emission induced by the STED beam. The collected signal is typically negligible due to efficient de-excitation and confocality of detection, but even the finite occupation of the excited state under a strong photon flux, with many molecules repeatedly transferred between the ground and excited electronic state, results in significant photobleaching. Moreover, the registered dependence of photobleaching vs. STED intensity, and thus optimal pulse duration, is strongly affected by the mechanism of STED-light excitation of fluorophores (one- vs. two-photon process). This mechanism of excitation is difficult to access experimentally due to the involvement of stimulated emission. Based on modelling (Fig. 3a), we conclude that two-photon absorption of the STED light by ATTO647N is negligible at the peak powers accessed in all STED experiments to date (up to $\sim 20 \mathrm{~W}$, Supplementary Fig. S9), and that the dominant mechanism of its STED-light-induced fluorescence is by linear absorption. For STED implementations with de-excitation pulses of a few hundred picoseconds, resolutions down to $\sim 30-40 \mathrm{~nm}$ have been reported for red dyes and nano-beads (Supplementary Fig. S9). A possible explanation why further resolution increases, requiring higher STED photon flux, have not been realized with sub-nanosecond pulses, lies in the pronounced intensity-dependent photobleaching contribution under STED conditions (as experiments were not power-limited). In contrast, current STED implementations with $\sim 1 \mathrm{~ns}$ pulses have allowed to reach the $\sim 20 \mathrm{~nm}$ resolution level by delivering more photons at the same peak power.

The majority of bleaching for $\sim 1$ ns pulses with red dyes at high STED pulse energies is expected to be mediated by one-photon absorption events of the STED light (based on the room-temperature absorption cross-section at the STED wavelength $750 \mathrm{~nm}$ ). This means that even the outermost parts of the STED doughnut drive bleaching (Fig. $5 \mathrm{f}$ and Supplementary Fig. S7c). The definition of the intensity zero (minimum) becomes even more important in order not to diminish the signal by off-switching at the targeted coordinate (Fig. 5e). Longer dwell times to accumulate more signal are accompanied by increased bleaching.

We have shown the spatial dependence of bleaching with respect to the targeted coordinate (Fig. 5b,f), which may prompt other insights. For example, applying a scanning scheme like MINFIELD ${ }^{17}$ will favour short durations of de-excitation pulses, as high-order photobleaching is already reduced by avoiding the exposure of molecules to the high intensity at the doughnut crest and periphery. 
In summary, our experiments confirm that optimal de-excitation occurs for STED pulse durations of few tens of picoseconds, as molecules are switched off quickly before spontaneous relaxation by fluorescence takes place. Such comparatively short pulses, while featuring the best de-excitation, come with increased high-order bleaching, which plays a significant role in STED at high intensities, as our measurements indicate. High-resolution STED requires sufficient photon flux in the direct vicinity of the targeted coordinate to define the "on"- "off" contrast. This causes high-order photobleaching associated with excited states of the fluorophore, mainly outside the target coordinate. High-order bleaching can be controlled by providing the de-excitation photons in longer STED pulses, a strategy pursued over the past years of STED development. The success of this approach depends on the magnitude of low-order photobleaching, for which triplet-quencher or dark-state relaxation strategies exist. The application of longer STED pulses will have to be combined with suitable gating to maintain maximal resolution. Based on our experimental data, the recently shown superior resolution for long, nanosecond STED pulses can be rationalised, as it reduced the high-order bleaching.

\section{References}

1. Hell, S. W. \& Wichmann, J. Breaking the diffraction resolution limit by stimulated emission: stimulated-emission-depletion fluorescence microscopy. Opt. Lett. 19, 780-782 (1994).

2. Göttfert, F. et al. Coaligned Dual-Channel STED Nanoscopy and Molecular Diffusion Analysis at $20 \mathrm{~nm}$ Resolution. Biophys. J. 105 L01-L03 (2013).

3. Westphal, V., Lauterbach, M. A., Di Nicola, A. \& Hell, S. W. Dynamic far-field fluorescence nanoscopy. New. J. Phys. 9, 435 (2007).

4. Westphal, V. et al. Video-Rate Far-Field Optical Nanoscopy Dissects Synaptic Vesicle Movement. Science 320, 246-249 (2008).

5. Schneider, J. et al. Ultrafast, temporally stochastic STED nanoscopy of millisecond dynamics. Nat. Methods 12, 827-830 (2015).

6. Hell, S. W. Microscopy and its focal switch. Nat. Methods 6, 24-32 (2009).

7. Kolmakov, K. et al. Red-Emitting Rhodamines with Hydroxylated, Sulfonated, and Phosphorylated Dye Residues and Their Use in Fluorescence Nanoscopy. Chem. Eur. J. 18, 12986-12998 (2012).

8. Wurm, C. A. et al. Novel red fluorophores with superior performance in STED microscopy. Opt. Nanoscopy 1, 7 (2012).

9. Pawley, J. B. Handbook of Biological Confocal Microscopy. 3rd edn. (Springer, 2006).

10. Song, L. L., Varma, C., Verhoeven, J. W. \& Tanke, H. J. Influence of the triplet excited state on the photobleaching kinetics of fluorescein in microscopy. Biophys. J. 70, 2959-2968 (1996).

11. Dyba, M. \& Hell, S. W. Photostability of a fluorescent marker under pulsed excited-state depletion through stimulated emission. Appl. Opt. 42, 5123-5129 (2003).

12. Donnert, G. et al. Macromolecular-scale resolution in biological fluorescence microscopy. Proc. Natl. Acad. Sci. USA 103, 11440-11445 (2006).

13. Donnert, G., Eggeling, C. \& Hell, S. W. Major signal increase in fluorescence microscopy through dark-state relaxation. Nat. Methods 4, 81-86 (2007).

14. Wu, Y. et al. Resonant Scanning with Large Field of View Reduces Photobleaching and Enhances Fluorescence Yield in STED Microscopy. Sci. Rep. 5, 14766 (2015).

15. Hoebe, R. A. et al. Controlled light-exposure microscopy reduces photobleaching and phototoxicity in fluorescence live-cell imaging. Nat. Biotechnol. 25, 249-253 (2007).

16. Staudt, T. et al. Far-field optical nanoscopy with reduced number of state transition cycles. Opt. Express 19, $5644-5657$ (2011).

17. Göttfert, F. et al. Strong signal increase in STED fluorescence microscopy by imaging regions of subdiffraction extent. Proc. Natl. Acad. Sci. USA 114, 2125-2130 (2017).

18. Rittweger, E., Han, K. Y., Irvine, S. E., Eggeling, C. \& Hell, S. W. STED microscopy reveals crystal colour centres with nanometric resolution. Nat. Photon. 3, 144-147 (2009).

19. Wildanger, D. et al. Solid Immersion Facilitates Fluorescence Microscopy with Nanometer Resolution and Sub-Angström Emitter Localization. Adv. Mater. 24, 309-313 (2012).

20. Kolmakov, K. et al. A Versatile Route to Red-Emitting Carbopyronine Dyes for Optical Microscopy and Nanoscopy. Eur. J. Org. Chem. 2010, 3593-3610 (2010).

21. Chirico, G., Cannone, F., Baldini, G. \& Diaspro, A. Two-Photon Thermal Bleaching of Single Fluorescent Molecules. Biophys. J. 84, 588-598 (2003).

22. Hell, S. W. et al. Stimulated emission on a microscopic scale: Light quenching of pyridinium 2 using a Ti:sapphire laser. J. Microsc. 180, RP1-RP2 (1995).

23. Schrader, M. et al. Monitoring the excited state of a dye in a microscope by stimulated emission. Bioimaging 3, 147-153 (1995).

24. Willig, K. I., Harke, B., Medda, R. \& Hell, S. W. STED microscopy with continuous wave beams. Nat. Methods 4, 915-918 (2007).

25. Moffitt, J. R., Osseforth, C. \& Michaelis, J. Time-gating improves the spatial resolution of STED microscopy. Opt. Express 19, 4242-4254 (2011).

26. Vicidomini, G. et al. Sharper low-power STED nanoscopy by time gating. Nat. Methods 8, 571-573 (2011).

27. Leutenegger, M., Eggeling, C. \& Hell, S. W. Analytical description of STED microscopy performance. Opt. Express 18, 26417-26429 (2010).

28. Kalies, S., Kuetemeyer, K. \& Heisterkamp, A. Mechanisms of high-order photobleaching and its relationship to intracellular ablation. Biomed. Opt. Express 2, 805-816 (2011).

29. Patterson, G. H. \& Piston, D. W. Photobleaching in two-photon excitation microscopy. Biophys. J. 78, 2159-2162 (2000).

30. Hell, S. W. \& Schönle, A. In Science of Microscopy Vol. 2 (eds Spence, J. C. H. \& Hawkes, P. W.) 790-834 (Springer, 2007).

31. Eggeling, C. et al. Analysis of photobleaching in single-molecule multicolor excitation and Förster resonance energy transfer measurements. J. Phys. Chem. A 110, 2979-2995 (2006)

32. Eggeling, C., Widengren, J., Rigler, R. \& Seidel, C. A. M. Photobleaching of fluorescent dyes under conditions used for singlemolecule detection: Evidence of two-step photolysis. Anal. Chem. 70, 2651-2659 (1998).

33. Hotta, J. et al. Spectroscopic Rationale for Efficient Stimulated-Emission Depletion Microscopy Fluorophores. J. Am. Chem. Soc. 132, 5021-5023 (2010)

34. Eggeling, C., Willig, K. I., Sahl, S. J. \& Hell, S. W. Lens-based fluorescence nanoscopy. Q. Rev. Biophys. 48, 178-243 (2015).

\section{Acknowledgements}

This work was supported by the Foundation for Polish Science International Ph.D. Projects Programme, cofinanced by the EU European Regional Development Fund.

\section{Author Contributions}

J.O. designed and performed experiments, analysed data and performed simulations. V.W. and C.R. advised on experimental aspects during the early phase of the project. J.O. and S.J.S. discussed all experimental and simulation data, and wrote the manuscript with input from S.W.H. who provided guidance throughout the project. 


\section{Additional Information}

Supplementary information accompanies this paper at doi:10.1038/s41598-017-09902-X

Competing Interests: S.W.H. owns shares of the companies Abberior and Abberior Instruments GmbH, producing super-resolution STED dyes and microscopes, respectively.

Publisher's note: Springer Nature remains neutral with regard to jurisdictional claims in published maps and institutional affiliations.

(c) (i) Open Access This article is licensed under a Creative Commons Attribution 4.0 International License, which permits use, sharing, adaptation, distribution and reproduction in any medium or format, as long as you give appropriate credit to the original author(s) and the source, provide a link to the Creative Commons license, and indicate if changes were made. The images or other third party material in this article are included in the article's Creative Commons license, unless indicated otherwise in a credit line to the material. If material is not included in the article's Creative Commons license and your intended use is not permitted by statutory regulation or exceeds the permitted use, you will need to obtain permission directly from the copyright holder. To view a copy of this license, visit http://creativecommons.org/licenses/by/4.0/.

(c) The Author(s) 2017 


\title{
Supplementary Information
}

\section{Photobleaching in STED nanoscopy and its dependence on the photon flux applied for reversible silencing of the fluorophore}

\author{
Joanna Oracz ${ }^{1,2, *}$, Volker Westphal ${ }^{1}$, Czesław Radzewicz ${ }^{2}$, Steffen J. Sahl ${ }^{1}$, and Stefan W. Hell ${ }^{1,3, *}$
}

${ }^{1}$ Max Planck Institute for Biophysical Chemistry, Department of NanoBiophotonics, Am Fassberg 11, 37077

Göttingen, Germany

${ }^{2}$ University of Warsaw, Faculty of Physics, Pastera 5, 02-093 Warsaw, Poland

${ }^{3}$ Max Planck Institute for Medical Research, Department of Optical Nanoscopy, Jahnstr. 29, 69120 Heidelberg,

Germany

*Correspondence should be addressed to S.W.H. (shell@mpibpc.mpg.de) or J.O. (joracz@mpibpc.mpg.de).

\section{Contents}

1 Temporal shaping of STED pulses $\quad$ S2

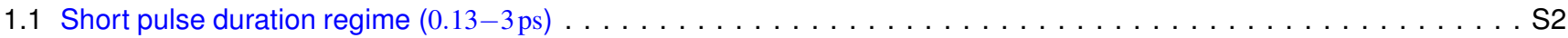

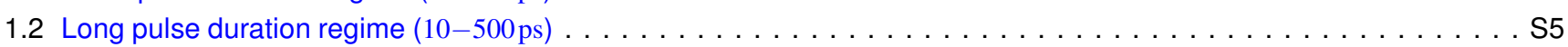

2 Data analysis $\quad S 5$

3 Influence of the STED pulse chirp S6

4 Simulation of de-excitation, bleaching and STED-light-induced fluorescence for ATTO647N S8

$4.13 \mathrm{D}$ fitting procedure to experimental data $\ldots \ldots \ldots \ldots \ldots \ldots$

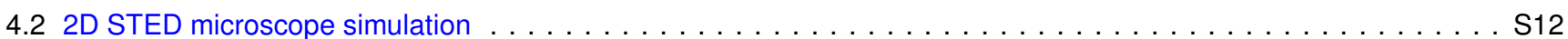

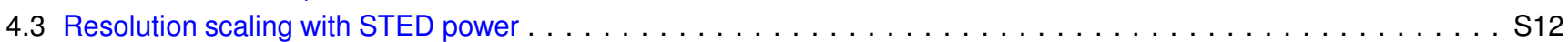

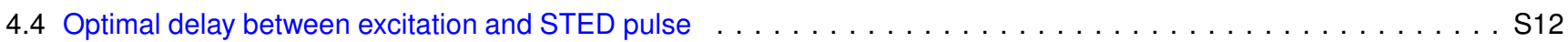

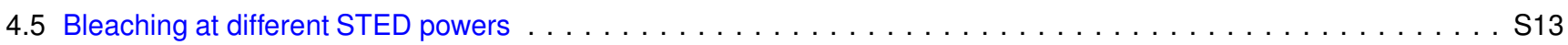

5 Simulation of de-excitation, bleaching and STED-light-induced fluorescence for ATTO590 S14

6 Comparison: Resolution in STED microscopy reported for red dyes and fluorescent beads S16

Supplementary References $\quad \mathrm{S17}$ 


\section{Temporal shaping of STED pulses}

\subsection{Short pulse duration regime $(0.13-3 \mathrm{ps})$}

\section{Characterization of the STED pulses at the back aperture of the objective lens}

To obtain roughly transform-limited ultrashort pulses at the back aperture of the objective lens, we aligned the home-built pulse compressor to measure the shortest possible pulse duration $(\sim 130 \mathrm{fs}$, data not shown $)$ with a commercial autocorrelator (pulseCheck, APE). Next, we characterized the spectral phase introduced by the home-built pulse shaper. We compared the phase shift imparted at the spatial light modulator (SLM) on a femtosecond pulse with an unmodified replica of the same pulse ("reference pulse") using Fourier transform spectral interferometry (SI) ${ }^{1}$. The relative delay between two pulses $t$ was controlled by the optical delay line (DL) placed in one of the arms of a Mach-Zehnder interferometer (Supplementary Fig. $\mathrm{S} 1 \mathrm{a})$. We registered the interference spectrum $\left(S_{S I}\right)$ of the pulses on a high-resolution spectrometer (500M, Spex), to resolve the high-frequency components over the narrow laser bandwidth (Supplementary Fig. S1b, FWHM $\approx 8.5 \mathrm{~nm}$ ). The registered spectrum $S_{S I}$ is described by:

$$
S_{S I}(\omega)=2 S(\omega)(1+\cos (\Delta \varphi+\omega t))
$$

where $S(\omega)$ is the spectral amplitude of the reference and shaped pulse, $\Delta \varphi$ is the relative spectral phase between them, and $t$ is the time delay. An example of a measured spectrum is presented in Supplementary Fig. S1b. After applying the Fourier transform, the modulated part can be distinguished in the side lobes (with separation proportional to pulse delay $t$ ). The relative spectral phase can be retrieved by the inverse Fourier Transform from one of either lobe signals. The example of a comparison between applied and retrieved $3^{\text {rd }}$-order spectral phase $\left(\varphi_{3}=3 \cdot 10^{6} \mathrm{fs}^{3}\right)$ is presented in Supplementary Fig. S1c, showing excellent agreement within the pulse spectrum.
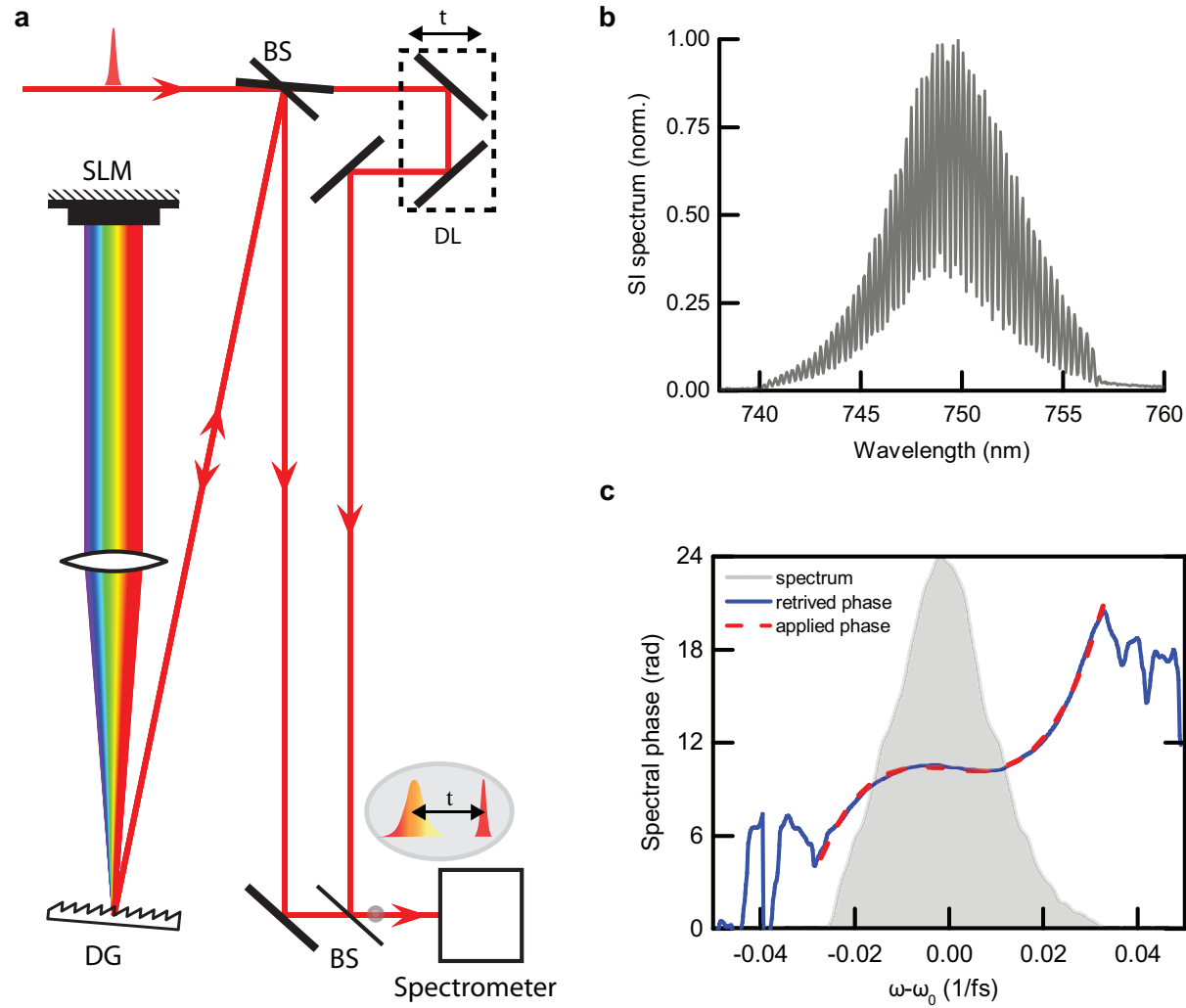

c

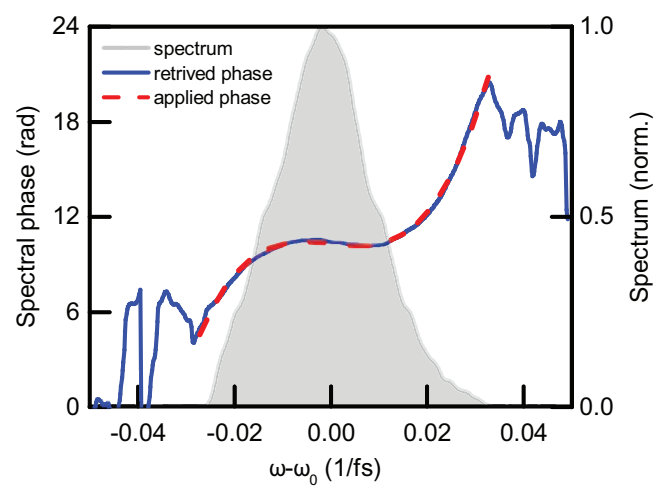

Supplementary Figure S1. Characterization of the pulse shaper by Fourier-transform spectral interferometry. (a) Schematic drawing of Mach-Zehnder interferometer with two copies of an ultrashort pulse created by a 50:50 low-dispersion non-polarizing beamsplitter (BS). One replica is shaped by the spatial light modulator (SLM) in the Fourier plane created by a diffraction grating (DG) and a long focal length lens $(f=400 \mathrm{~mm})$. The other pulse passes an optical delay line (DL) which controls the relative timing between two pulses $t$. The two pulses are combined by a second BS, and the modulated spectra are registered on the spectrometer. (b) Example of registered spectral interferometry (SI) data showing strong signal modulation $\left(\varphi_{3}=3 \cdot 10^{6} \mathrm{fs}^{3}\right)$. (c) Retrieved spectral phase of the pulse from spectrum presented in (b). The retrieved phase shows perfect agreement with the applied phase on the $\operatorname{SLM}\left(\varphi_{3}=3 \cdot 10^{6} \mathrm{fs}^{3}\right.$, red dashed line). 


\section{Characterization of the STED pulses in the focal plane}

The high-numerical-aperture objective lens introduces an additional spectral phase which smears out the temporal profile of an ultrashort pulse in the focal plane. We corrected this influence by an iterative procedure, monitoring two-photon excitation fluorescence (2PEF) in Coumarin 120 (Lambda Physik) dissolved in TDE. We iteratively maximized the 2PEF signal by changing the spectral phase on the shaper up to the $4^{\text {th }}$ order. The final correction phase is presented in Supplementary Fig. S2a. Next, we measured the autocorrelation function (ACF) in the focal plane in the same Coumarin 120 solution, before and after the objective phase correction (Supplementary Fig. S2b). For corrected spectral phase, we observed higher signal and a narrower width of the ACF. A slightly asymmetrical ACF for the uncorrected pulse is related to spatial misalignment between the two pulses. The ACF width indicated a pulse duration of $\sim 130 \mathrm{fs}$ in the focal plane, corresponding perfectly to the measured transform-limited pulse ACF at the back aperture by a commercial autocorrelator (data not shown). To explore, we also applied more complicated spectral phases to the STED pulse, to generate, from a single femtosecond pulse ( $\sim 130 \mathrm{fs})$, a burst of 9 pulses, each with $\sim 130$ fs pulse duration separated by $\sim 500 \mathrm{fs}$ (peak to peak). We calculated the necessary spectral phase for such an intensity profile by the iterative Fast Fourier Transform algorithm ${ }^{2}$. The spectral phase retrieved by SI and the expected temporal profile are presented in Supplementary Fig. S2c. To verify the temporal profile of the pulse in the focal plane, we measured the ACF as before, finding perfect agreement with the expected distribution (Supplementary Fig. S2d). The duration of the Gaussian STED pulse was controlled by applying different $2^{\text {nd }}$-order spectral phases (chirp), according to the relation:

$$
\tau=\tau_{0} \sqrt{1+\left(4 \ln 2 \frac{\varphi_{2}}{\tau_{0}^{2}}\right)^{2}} \approx 4 \ln 2 \frac{\varphi_{2}}{\tau_{0}}
$$

where $\tau$ is the pulse duration, $\tau_{0}$ is the transform-limited pulse duration and $\varphi_{2}$ is a $2^{\text {nd }}$-order spectral phase (i.e. pulse chirp). An up-chirped pulse corresponds to instantaneous frequency increases with time $\left(\varphi_{2}>0\right)$, in down-chirped pulses frequency decreases with time $\left(\varphi_{2}<0\right)$. We measured the STED pulse duration $\tau$ in the focal plane for different chirp values $\varphi_{2}$ (Supplementary Fig. S2e, Supplementary Table S1), finding a linear relation. In the short-pulse regime, the time delay between excitation and STED pulse was measured by the crosscorrelation function (CCF) of the ultrashort STED pulse replica with the excitation and STED pulse in the focal plane (in Coumarin 120 solution). The example of a CCF of the STED (750 nm) and excitation $(635 \mathrm{~nm})$ pulse is presented in Supplementary Fig. S2f. These measurements allow to precisely place the STED pulse after the excitation by use of the DL and estimate the excitation pulse duration to $\sim 500 \mathrm{fs}$ in the focal plane.

Supplementary Table S1. Control of the STED pulse duration by the second-order spectral phase applied on the SLM. Autocorrelation function width (FWHM) as measured in the focal plane and deconvolved pulse duration (Gaussian, FWHM) for different $2^{\text {nd }}$ order spectral phases $\left(\varphi_{2}\right)$.

\begin{tabular}{ccc}
\hline Spectral phase $\varphi_{2}\left(\mathrm{fs}^{2}\right)$ & ACF width $(\mathrm{ps})$ & pulse duration $\tau(\mathrm{ps})$ \\
\hline 0 & 0.179 & 0.127 \\
$3 \cdot 10^{4}$ & 1.168 & 0.826 \\
$6 \cdot 10^{4}$ & 2.276 & 1.626 \\
$9 \cdot 10^{4}$ & 3.522 & 2.516 \\
\hline
\end{tabular}


a

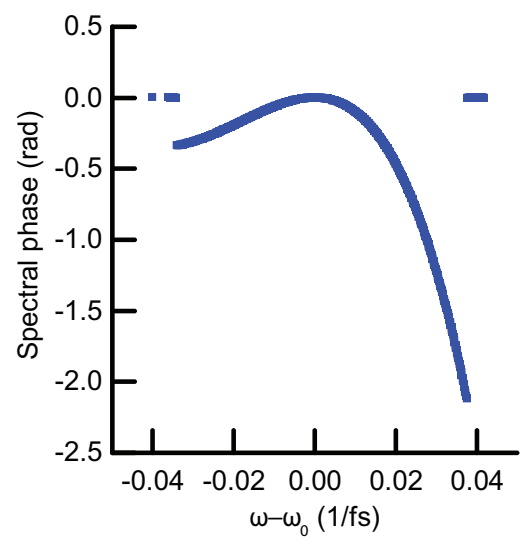

c Burst pulse generation
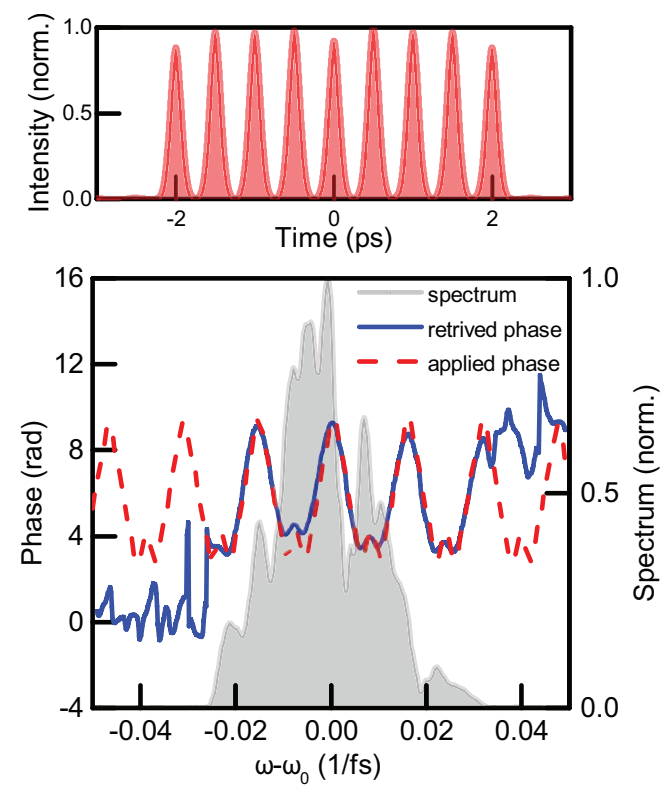

e Pulse duration in the focal plane

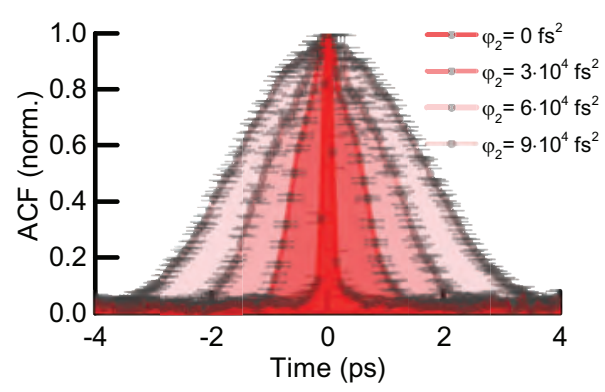

b ACF in the focal plane

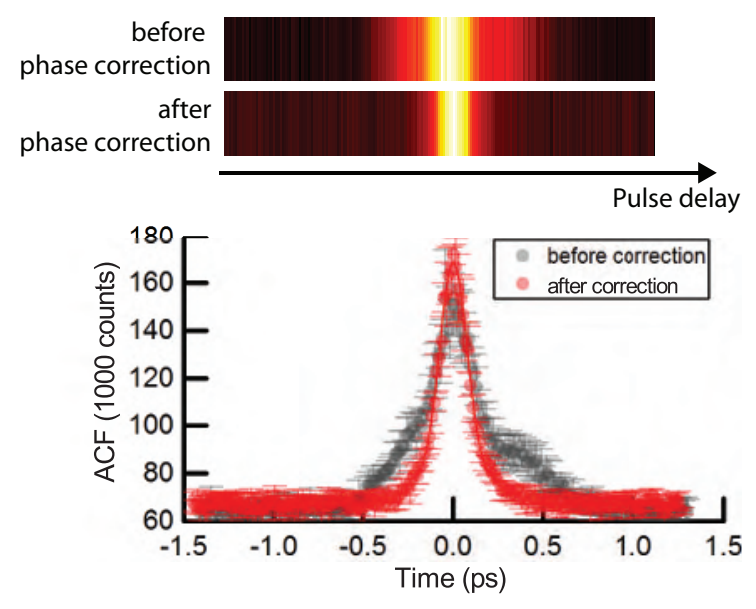

d ACF in the focal plane
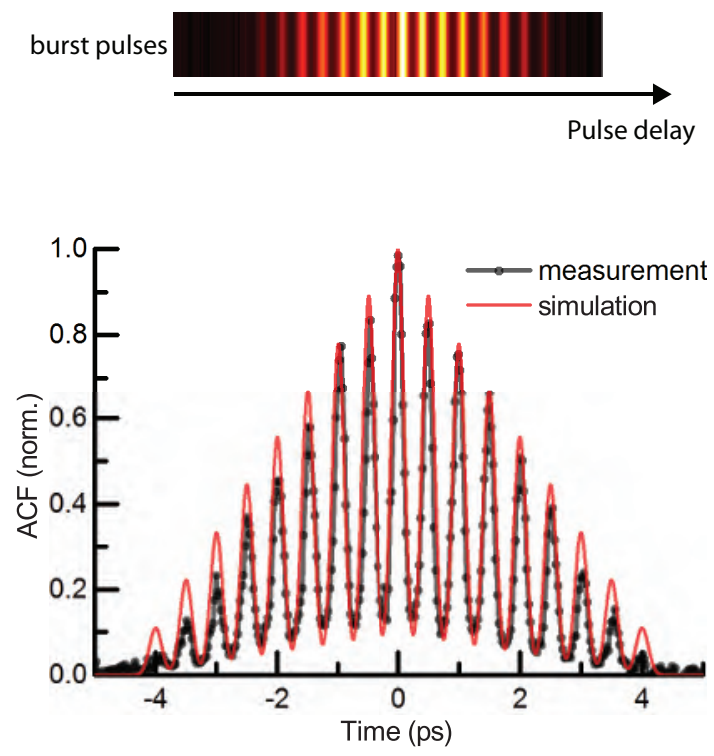

f Excitation and STED pulse delay

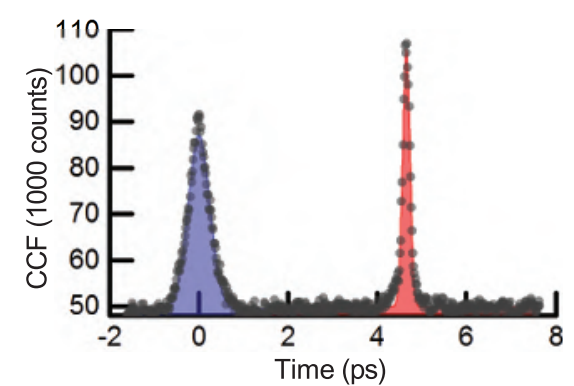

Supplementary Figure S2. STED pulse characterization in the ultrashort pulse regime (0.13-3 ps). (a) Retrieved correction spectral phase for the objective lens. (b) Autocorrelation function (ACF) of an ultrashort pulse in the focal plane before and after the objective phase correction. The ACF was measured in Coumarin 120 solution by two-photon excitation fluorescence. (c) Generation of burst pulses from a single femtosecond pulse. Expected temporal intensity profile (top) and applied/retrieved spectral phase (bottom). (d) Measured ACF in the focal plane for burst pulses. The red line represents the expected ACF for burst pulses with the temporal profile shown in (c). (e) Control of the STED pulse duration in the focal plane by $2^{\text {nd }}$-order spectral phase $\varphi_{2}$. Intensity profiles represent the measured ACF in the focal plane. (f) Relative delay between excitation and STED pulse as measured by the crosscorrelation function (CCF) in the focal plane. 


\subsection{Long pulse duration regime (10-500ps) Characterization of STED pulse duration by autocorrelation}

The pulse duration of long STED pulses was measured by a commercial autocorrelator (pulseCheck, APE) at the back aperture of the objective lens (Supplementary Fig. S3). The spectral phase introduced by the objective lens is negligible in this pulse duration regime. Before coupling the STED pulses to optical fibers, several high-dispersion glass rods were placed in the optical path to pre-stretch the ultrashort pulses and thus minimize nonlinear effects in the fibers. Different amounts of chirp were applied to the pulses by changing the length of polarization-maintaining fibers (Supplementary Table S2). The longest pulse duration $(\sim 500 \mathrm{ps})$ was at the edge of the autocorrelator measurement range. The time delay between excitation and STED pulse was controlled electronically to be approximately the FWHM of the STED pulse duration to maintain the highest possible de-excitation.

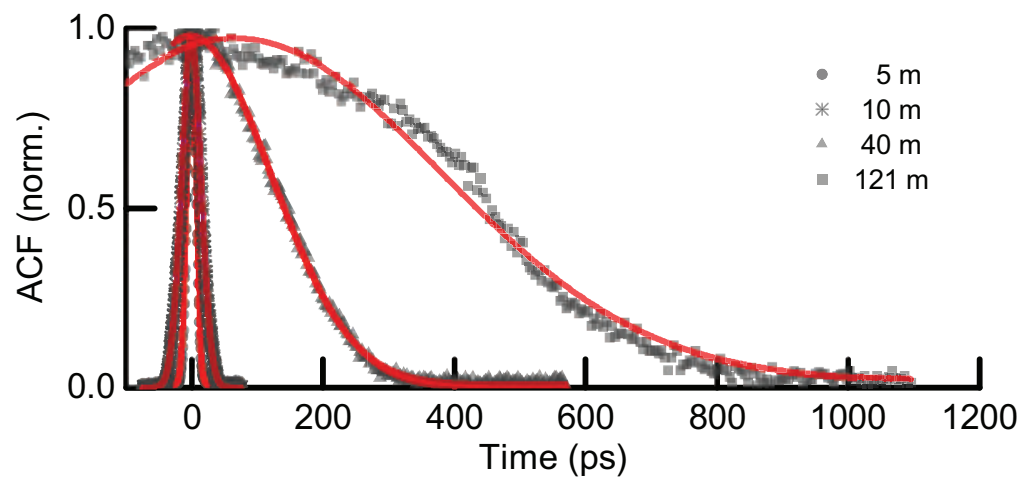

Supplementary Figure S3. STED pulse characterization in the long pulse regime (10-500 ps). Autocorrelation function (ACF), as measured at the back aperture of the objective lens for different lengths of the optical fibers. Red lines represent the Gaussian fits.

Supplementary Table S2. STED pulse duration with different optical fibers. Autocorrelation function width (ACF, FWHM), as measured at back aperture of the objective lens, and deconvolved STED pulse duration (Gaussian, FWHM) for different optical fibers. (*) Measurements for the longest pulses were at the edge of the autocorrelator range.

\begin{tabular}{llll}
\hline Fiber length $(\mathrm{m})$ & Model, manufacturer & ACF width $(\mathrm{ps})$ & pulse duration $\tau(\mathrm{ps})$ \\
\hline 5 & PM630-HP, Thorlabs & 16 & 11 \\
10 & PM630-HP, Thorlabs & 34 & 25 \\
40 & PMC-600-4, Schäfter + Kirchhoff GmbH & 283 & 200 \\
121 & PMJ-A3AHPC, OZ Optics & 736 & $520^{*}$ \\
\hline
\end{tabular}

\section{Data analysis}

An example of raw data from a measurement for a single pulse configuration is presented in Supplementary Fig. S4. First, we measured the reference fluorescence signal of the excitation pulse (Supplementary Fig. S4a). Then, the signal for the excitation and chopped STED beams acting together (Supplementary Fig. S4b) was measured and, finally, fluorescence induced by the chopped STED beam acting alone on the molecules (Supplementary Fig. S4c). Each measurement averaged signal over $\sim 100 \mathrm{~s}$. The respective time traces are presented in Supplementary Fig. S4d. In the data analysis process, we normalized all curves to the initial fluorescence signal $C_{0}$ (excitation only) (Supplementary Fig. S4a). Then, we subtracted fluorescence induced by the STED beam $C_{S}$ from the signal during exposure to both the excitation and STED beams together $C_{E S}$ (Supplementary Fig. S4b minus Supplementary Fig. S4c). Next, we distinguished the fast fluorescence recovery component (de-excitation) from the slow recovery part (diffusion of fresh molecules, i.e., proportional to photobleaching) by an automated procedure incorporating the derivative of the second curve (Supplementary Fig. S4d, blue line). De-excitation, bleaching and STED-light-induced fluorescence were calculated as mean values from $>10$ recovery traces (Supplementary Fig. S4d). The error of measurement is estimated from the standard deviation of retrieved values. It is important to note that, for the same sample, often different offsets of photobleaching (low-order photobleaching) were observed due to aging, probably related to changes in oxygen concentration. For this reason, all measurements were performed in freshly prepared solutions. The relative dependence on the pulse duration (high-order photobleaching) was characterized by the same order of nonlinearity in all cases. 
a

b
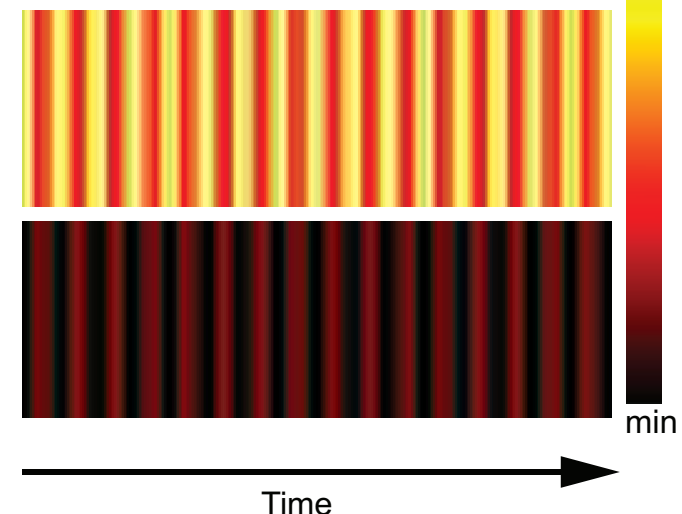

$\max$

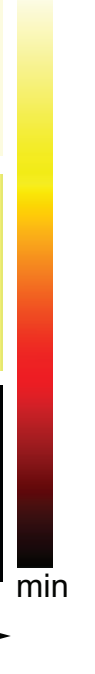

d

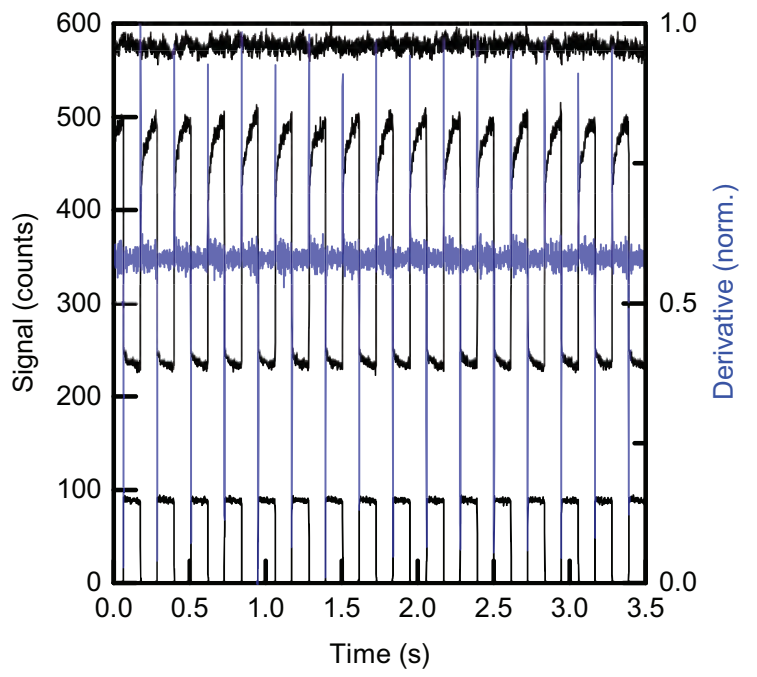

Supplementary Figure S4. Raw data (here: CW STED in ATTO647N TDE solution, $0.5 \mu$ M concentration, average over $\sim 100 \mathrm{~s}$.) (a) Fluorescence signal due to excitation pulses $(635 \mathrm{~nm}, 30 \mu \mathrm{W}$ average power). (b) Fluorescence recovery signal for excitation pulse (as previously) and chopped STED $(750 \mathrm{~nm}, 150 \mathrm{~mW}$ average power). (c) Fluorescence signal induced by the chopped STED beam (as previously). (d) Intensity traces of (a-c). The blue line represents the derivative of (b).

\section{Influence of the STED pulse chirp}

We observed a noticeable dependence of bleaching and STED-light-induced fluorescence on the spectral chirp of the deexcitation pulse. For all tested dyes and peak intensities, down-chirped STED pulses showed 25\% lower STED-induced fluorescence and $\sim 10 \%$ lower bleaching in comparison to commonly used up-chirped de-excitation pulses (Supplementary Fig. S5b,c). Within the measurement error, de-excitation was the same in both cases.

The influence of the chirp can be rationalized in the wave packet propagation picture (Supplementary Fig. S5a). Assuming two electronic potential surfaces $V_{0}, V_{1}$, the optical transition between them is possible only in case of resonance with an instantaneous optical frequency. After an ultrafast excitation, the wave packet propagates on an excited state potential $\left(V_{1}\right)$ and quickly relaxes by vibrational relaxation $(\sim 1 \mathrm{ps})$ to the lowest energy level of $V_{1}$. Incoming de-excitation photons cause stimulated emission. The energy difference of the wave packet after de-excitation and excited state potential $\left(V_{1}-V_{0}\right)$ increases in time, and an up-chirped pulse follows this motion causing unwanted excitation by the STED light. A down-chirped pulse gives better results, as STED-light-mediated excitation is reduced, due to the opposite temporal distribution of spectral components within the pulse. This process will be more prominent for pulses with higher energy and was observed before in the context of efficient excitation in fluorescent dye solutions ${ }^{3,4}$. 
a

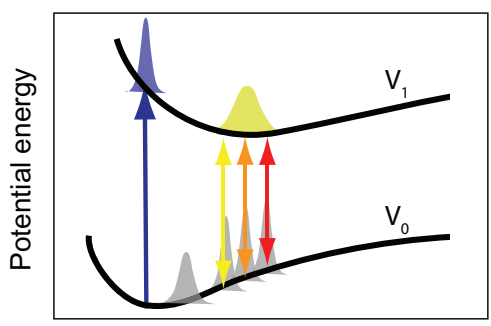

Interatomic distance
Down-chirped pulse $\left(\varphi_{2}<0\right)$

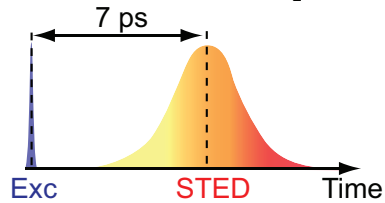

Up-chirped pulse $\left(\varphi_{2}>0\right)$

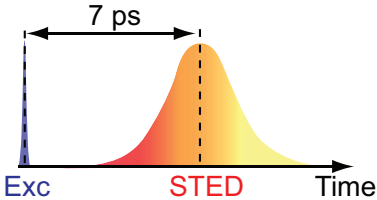

b

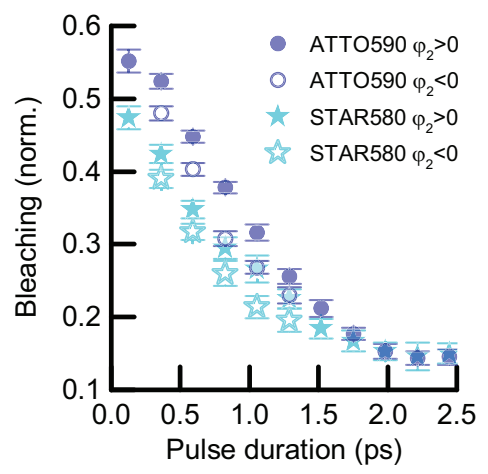

C

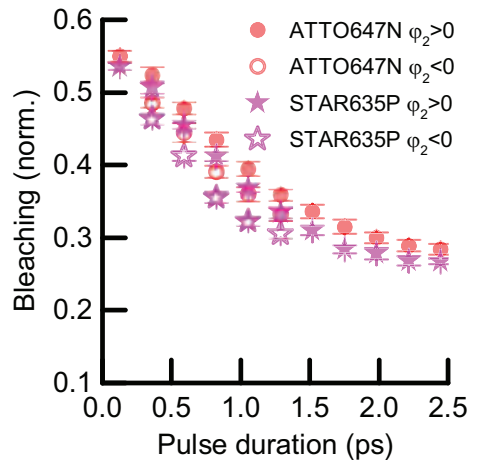

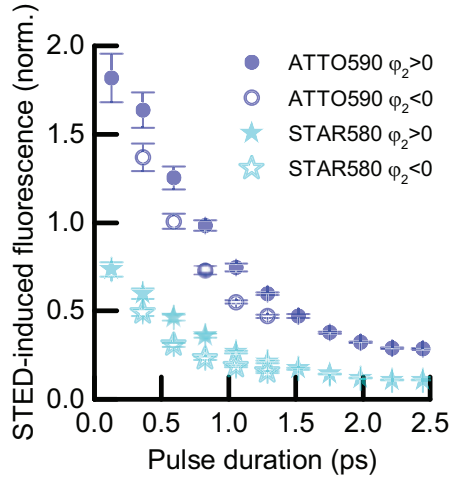
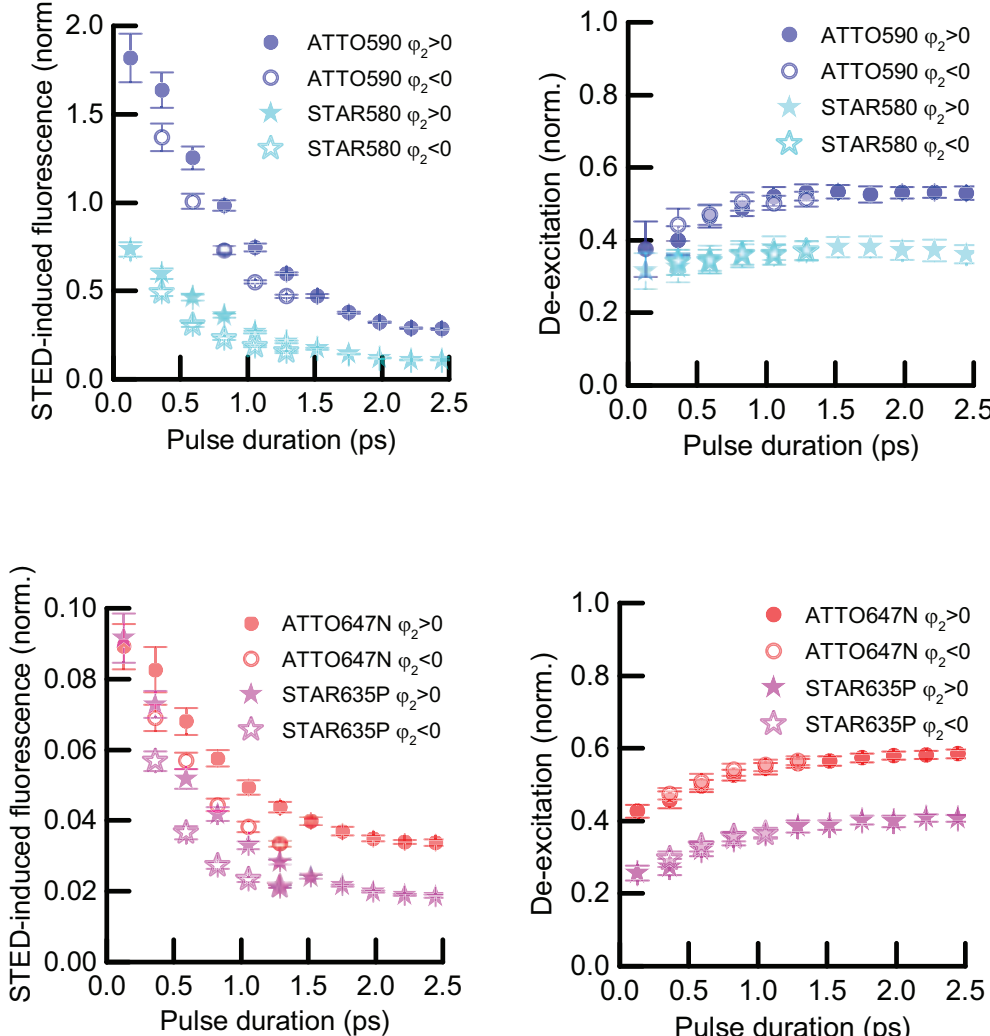

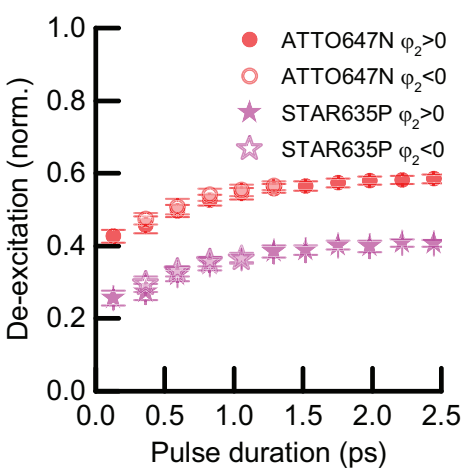

Supplementary Figure S5. Influence of the STED pulse chirp on the population of the excited state and photobleaching. (a) Schematic illustration of wave packet motion after de-excitation. The energy difference of the wave packet just after de-excitation and the excited-state potential $\left(V_{1}-V_{0}\right)$ increases in time, and an up-chirped pulse follows this motion, causing undesirable excitations of the just de-excited molecules. (b) Bleaching, STED-light-induced fluorescence and de-excitation as a function of pulse duration and chirp of the STED pulse for the dyes ATTO590 and STAR580. (c) Data as in (b) for the red dyes ATTO647N and STAR635P. All measurements were performed for an excitation power of $30 \mu \mathrm{W}$ and a STED power of $10 \mathrm{~mW}$ (measured at the back aperture of the objective lens). 


\section{Simulation of de-excitation, bleaching and STED-light-induced fluorescence for ATT0647N}

\subsection{D fitting procedure to experimental data}

To model the behaviour of ATTO647N in STED microscopy for various STED photon fluxes we inferred the necessary probabilities of the involved processes (i.e. stimulated emission $\sigma_{S T E D}$, one/two-photon STED light absorption $\sigma_{1 P E} / \sigma_{2 P E}$ and photobleaching probability $k$ ) based on experimental data (Fig. 3a). We applied a 3D fitting procedure to include the 3D spatial profiles of the beams, and thus obtain more realistic parameters.

De-excitation $(D)$, bleaching $(B)$ and STED-light-induced fluorescence $(S F)$ are defined as in the experiments:

$$
\begin{aligned}
D & =1-\left(\frac{\sum_{x, y, z} \overline{S_{1}(t)}}{\sum_{x, y, z} \overline{S_{1}(t)} \mid I_{S T E D}=0}-S F\right) \\
B & =\frac{\sum_{x, y, z} \overline{\beta(t)}}{\sum_{x, y, z} \overline{S_{1}(t)} \mid I_{S T E D}=0} \\
S F & =\frac{\sum_{x, y, z} \overline{S_{1}(t)} \mid I_{E x c}=0}{\sum_{x, y, z} \overline{S_{1}(t)} \mid I_{S T E D}=0}
\end{aligned}
$$

\section{Temporal domain}

The simplified model is presented in Supplementary Fig. S6. Most parameters are chosen as standard for fluorescent dyes and are fixed in the model (fluorescence lifetime $\tau_{f l}$, vibrational relaxation $\gamma_{v i b}$, excitation cross-section $\sigma_{e x c}$, see Supplementary Table S3). The excitation pulse has a Gaussian shape with constant pulse duration $\tau_{\text {exc }}=500 \mathrm{fs}$ and position $t_{0}=1100 \mathrm{ps}$ (Supplementary Fig. S6b). The STED pulse properties (duration $\tau$, position $t$, average power $P_{S T E D}$ ) are varied according to experimental conditions. The numerical time axis has length $t_{\text {axis }}=13 \mathrm{~ns}$, which roughly corresponds to one cycle at the $f=80 \mathrm{MHz}$ repetition rate. We assumed that all events on this time scale will be repeating with the next pulses (steady-state approximation). The rate equations derived from the diagram of simplified molecular states (Supplementary Fig. S6a) are presented below:

$$
\begin{aligned}
\frac{\mathrm{d}}{\mathrm{d} t} S_{1}{ }^{*} & =\left(w_{\text {exc }}+w_{\text {reexc }}\right)\left(S_{0}-S_{1}{ }^{*}\right)-\gamma_{v i b} S_{1}{ }^{*}-k\left(I_{\text {STED }}\right) S_{1}{ }^{*} \\
\frac{\mathrm{d}}{\mathrm{d} t} S_{1} & =\gamma_{v i b} S_{1}{ }^{*}-\gamma_{f l} S_{1}-w_{\text {STED }}\left(S_{1}-S_{0}{ }^{*}\right)-k\left(I_{\text {STED }}\right) S_{1} \\
\frac{\mathrm{d}}{\mathrm{d} t} S_{0}{ }^{*} & =w_{S T E D}\left(S_{1}-S_{0}{ }^{*}\right)+\gamma_{f l} S_{1}-\gamma_{v i b} S_{0}{ }^{*} \\
\frac{\mathrm{d}}{\mathrm{d} t} S_{0} & =\gamma_{v i b} S_{0}{ }^{*}-\left(w_{\text {exc }}+w_{\text {reexc }}\right)\left(S_{0}-S_{1}{ }^{*}\right) \\
\frac{\mathrm{d}}{\mathrm{d} t} \beta & =k\left(I_{S T E D}\right)\left(S_{1}+S_{1}{ }^{*}\right)
\end{aligned}
$$

where $\gamma=\frac{1}{\tau}, \tau$ is the lifetime of a state; $w=\frac{\sigma I}{\hbar \omega}$, with $\sigma$ a cross-section, $I$ the light intensity, and $\hbar \omega$ the photon energy; $k\left(I_{S T E D}\right)$ is the probability of photobleaching as a function of STED instantaneous intensity.

\section{Assumptions and free parameters in the model:}

(i) The population of each state is described by means of a probability, i.e. $\sum_{i} S_{i}=1$.

(ii) Initially all molecules are in the ground state $S_{0}=1$.

(iii) Excitation of ground-state molecules by the STED beam $\left(w_{\text {reexc }}\right)$ occurs by one- or two-photon absorption $\left(\sigma_{1 P E}, \sigma_{2 P E}\right)$ and $w_{\text {reexc }}=\sigma_{1 P E} \cdot \frac{I_{S T E D}}{\hbar \omega}+\frac{1}{2} \sigma_{2 P E} \cdot \frac{I_{S T E D}{ }^{2}}{\hbar \omega}$. The contributions of linear and two-photon absorption components have to be found experimentally. Note that in our simulation excitation by the STED beam occurs from the $S_{0}$ level, for simplicity. The more realistic situation for linear absorption at room temperature is presented in Fig. 5a. The interaction of molecules with the lower-energy STED-light photons leads to the population of higher vibrational levels of $S_{0}$, and is properly described by the Boltzmann distribution.

(iv) Bleaching by the excitation beam is not included in the model and negligible in comparison to STED-light-induced bleaching.

(v) Bleaching occurs only from the excited states $\left(S_{1}, S_{1}{ }^{*}\right)$ and is a monotonic function of STED intensity described as $k\left(I_{S T E D}\right)=k_{1} \cdot\left(\frac{I_{S T E D}}{1 W / m^{2}}\right)^{b}$, where $b$ is the order of nonlinearity of photobleaching. For ATTO647N, we measured $b=1.4$. The photobleaching amplitude $k_{1}$ has to be found experimentally.

(vi) The probability (cross-section) of stimulated emission $\sigma_{S T E D}$ has to be found experimentally. 


\section{Spatial domain}

Beam intensities in the foci are approximated by 3D Gaussian beams (Supplementary Fig. S6c):

$$
I(r, z)=\frac{2 P}{\pi w(z)^{2}} \exp \left(-\frac{2 r^{2}}{w(z)^{2}}\right)
$$

where $P$ is the average power, $w(z)=w_{0} \sqrt{1+\frac{z}{z_{R}}}$ is the beam radius at the axial position $z, w_{0}=\frac{F W H M}{\sqrt{2 \ln (2)}}$ is the beam radius in the focal plane, and $z_{R}=\frac{\pi w_{0}^{2}}{\lambda}$ is the Rayleigh range. Beam sizes are chosen as in the experiment, that is, the region of excitation in the focal plane is diffraction-limited $(\mathrm{FWHM}=227 \mathrm{~nm})$; the STED beam is enlarged $(\sim 1.5$ of diffraction-limited size at the STED wavelength, FWHM $=402 \mathrm{~nm}$ ). The confocal detection corresponds to $\sim 1$ Airy disc (AD) at the excitation wavelength.

\section{Extraction of free parameters from the experimental data}

All parameters employed in the model are listed in Supplementary Table S3. Values of free parameters $\left(\sigma_{1 P E}, \sigma_{2 P E}, \sigma_{S T E D}, k_{1}\right.$; highlighted in yellow) were found by scanning the parameter space and comparing simulated values to the experimental data (Fig. 3a). The normalized root mean square error (nRMSE) quantifies the quality of the global fit to the data. nRMSE was $\leq 10 \%$ for all three curves. The final modelled values of de-excitation, bleaching, and STED-light-induced fluorescence as a function of STED pulse duration $\tau$ are presented as solid lines in Fig. 3a.

Discussion of the inferred free parameter values:

(i) The deduced probability of stimulated emission $\left(\sigma_{S T E D}=4.8 \times 10^{-18} \mathrm{~cm}^{2}\right)$ is in good agreement with the expected stimulated emission cross-section for fluorescent dyes of order $10^{-17} \mathrm{~cm}^{2}$.

(ii) The linear absorption cross section of STED photons $\left(\sigma_{1 P E}=3.5 \times 10^{-21} \mathrm{~cm}^{2}\right)$ corresponds to the expected value at room temperature at the STED wavelength. In these conditions, higher vibrational levels of $S_{0}$ are occupied, and some molecules interact with the STED photons. The occupancy of higher vibrational states is described by the Boltzmann distribution, which well describes the red edge of the absorption band. Assuming that at an excitation wavelength $\lambda_{\text {exc }}=635 \mathrm{~nm}$ the absorption cross-section is $\sigma_{\text {exc }}\left(\lambda_{\text {exc }}\right) \approx 1 \times 10^{-16} \mathrm{~cm}^{2}$, the one-photon absorption probability at the STED wavelength $\left(\lambda_{S T E D}=750 \mathrm{~nm}\right)$ at room temperature $(T=293 \mathrm{~K})$ can be estimated as:

$$
\sigma_{1 P E} \approx \sigma_{e x c} \cdot \exp \left(-\frac{E\left(\lambda_{e x c}\right)-E\left(\lambda_{S T E D}\right)}{k T}\right) \approx 1.7 \cdot 10^{-5} \sigma_{e x c}=1.7 \times 10^{-21} \mathrm{~cm}^{2} .
$$

(iii) The two-photon excitation cross-section $\sigma_{2 P E}=3.5 G M$ roughly agrees with values measured for ATTO647N Streptavidin in phosphate buffered saline solution $\left(\sigma_{2 P E}=8 \pm 4 \mathrm{GM} \text { at } 750 \mathrm{~nm}\right)^{5}$. 
a
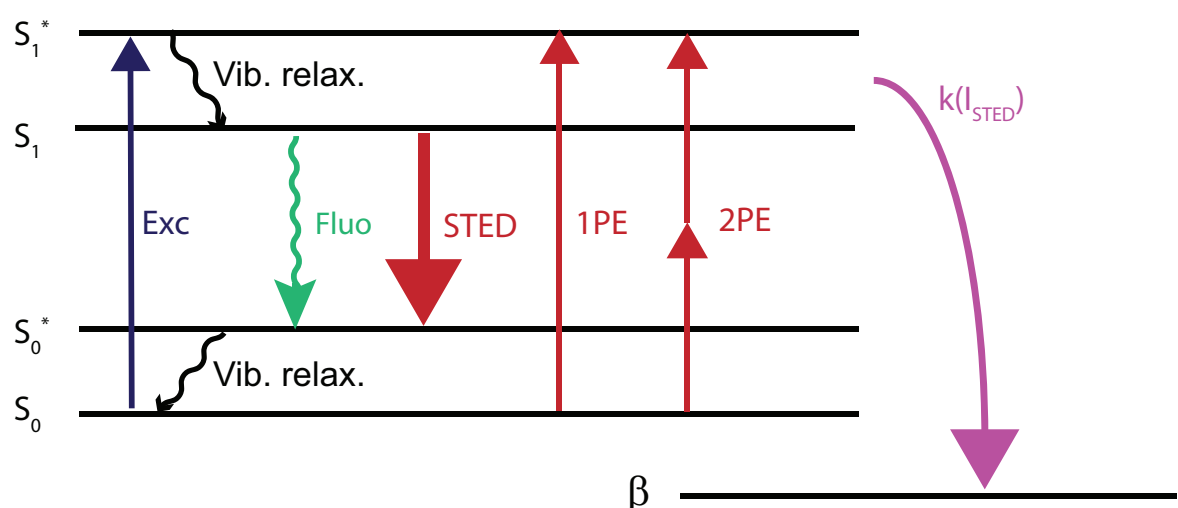

b
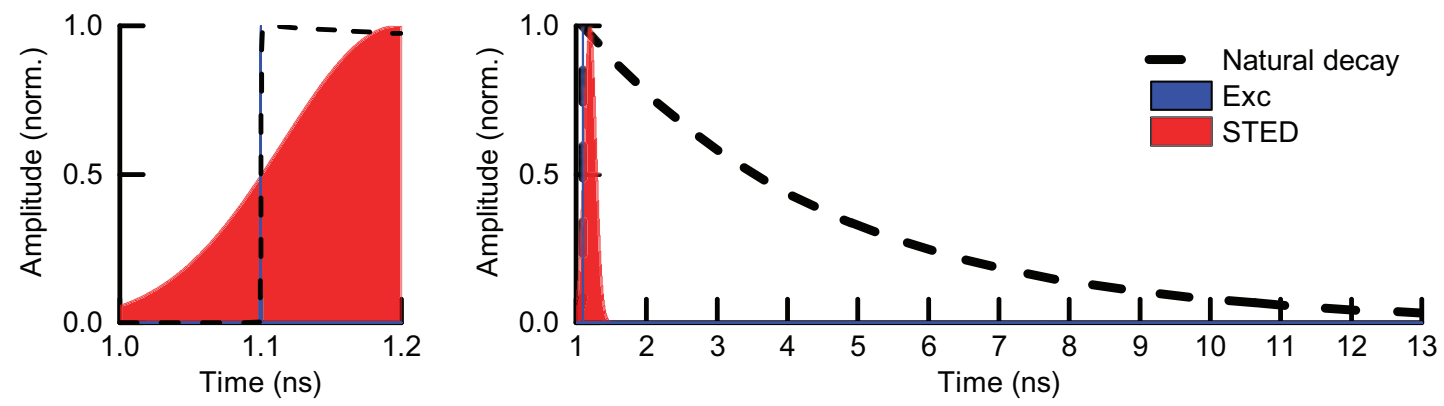

C
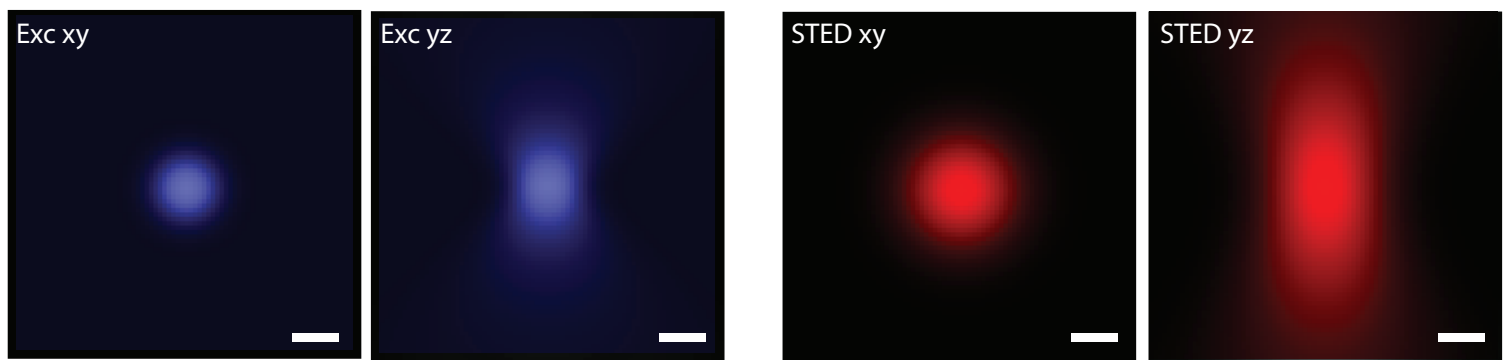

Supplementary Figure S6. Numerical simulation of de-excitation, bleaching and STED-light-induced fluorescence. (a) Simplified model of photobleaching in STED microscopy. (b) Temporal domain. Numerical time axis with excitation (blue) and STED (red) pulse profiles. The dashed line represents the natural fluorescence decay (i.e. STED power equals zero). (c) Spatial beam profiles for lateral ( $x y$ ) and axial $(y z)$ coordinates. All scale bars: $200 \mathrm{~nm}$. 
Supplementary Table S3. Parameters employed for modelling ATTO647N behaviour in STED nanoscopy. Fixed parameters are shown in white. Parameters extracted from fits to experimental data are highlighted in yellow. nRMSE represents a normalized root mean square error of the fit. The modelled curves and experimental data are presented in Fig. 3.

\begin{tabular}{|c|c|c|c|}
\hline \multicolumn{4}{|c|}{ Temporal domain } \\
\hline Parameter & Symbol & Value/Range & nRMSE \\
\hline time step & $\mathrm{d} t$ & $125 \mathrm{fs}$ & \\
\hline time range & $t_{\text {axis }}$ & $13 \mathrm{~ns}$ & \\
\hline vibrational relaxation & $t_{v i b}$ & $1 \mathrm{ps}$ & \\
\hline fluorescence lifetime & $\tau_{f l}$ & $3.5 \mathrm{~ns}$ & \\
\hline excitation cross-section & $\sigma_{e x c}$ & $1 \times 10^{-16} \mathrm{~cm}^{2}$ & \\
\hline excitation pulse duration & $\tau_{\text {exc }}$ & $500 \mathrm{fs}$ & \\
\hline excitation pulse position & $t_{\text {exc }}$ & $1.1 \mathrm{~ns}$ & \\
\hline excitation power & $P_{\text {exc }}$ & $30 \mu \mathrm{W}$ & \\
\hline stimulated emission cross-section & $\sigma_{S T E D}$ & $4.8 \times 10^{-18} \mathrm{~cm}^{2}$ & $2 \%$ \\
\hline STED pulse duration & $\tau$ & $0.5-3000 \mathrm{ps}$ & \\
\hline STED pulse position & $t_{S T E D}$ & $1.1 \mathrm{~ns}+0.75 \tau$ & \\
\hline STED power & $P_{S T E D}$ & $1-1000 \mathrm{~mW}$ & \\
\hline STED one-photon excitation & $\sigma_{1 P E}$ & $3.5 \times 10^{-21} \mathrm{~cm}^{2}$ & $10 \%$ \\
\hline STED two-photon excitation & $\sigma_{2 P E}$ & $3.5 \mathrm{GM}$ & \\
\hline photobleaching rate & $k_{1}(b=1.4)$ & $5.2 \times 10^{-10} \mathrm{~Hz}$ & $5 \%$ \\
\hline repetition rate & $f$ & $80 \mathrm{MHz}$ & \\
\hline \multicolumn{4}{|c|}{ Spatial domain } \\
\hline Parameter & Symbol & Value/Range & nRMSE \\
\hline radial step & $\mathrm{d} r$ & $20 \mathrm{~nm}$ & \\
\hline radial range & $r_{\text {range }}$ & $\pm 200 \mathrm{~nm}$ & \\
\hline axial step & $\mathrm{d} z$ & $20 \mathrm{~nm}$ & \\
\hline axial range & $z_{\text {range }}$ & $\pm 700 \mathrm{~nm}$ & \\
\hline excitation wavelength & $\lambda_{\text {exc }}$ & $635 \mathrm{~nm}$ & \\
\hline STED wavelength & $\lambda_{S T E D}$ & $750 \mathrm{~nm}$ & \\
\hline Factor of STED-focus enlargement & $M$ & 1.5 & \\
\hline Numerical aperture & NA & 1.4 & \\
\hline Confocal detection & conf & $1 \mathrm{AD}$ & \\
\hline
\end{tabular}




\subsection{D STED microscope simulation}

The 3D fitting procedure described above is costly in terms of time. In many practical cases, one can assume that the microscopic sample is two-dimensional (object is significantly thinner than the axial resolution, e.g. nuclear pore complexes). Calculations of resolution and spatial distribution of photobleaching were therefore performed for 2D intensity distributions, ignoring the axial dimension of the implemented profiles (Fig. 5, Supplementary Fig. S7). The radial step d $r$ for these numerical simulations was decreased from $20 \mathrm{~nm}$ to typically $2 \mathrm{~nm}$. In the focal plane, the excitation beam is approximated by a Gaussian function, and STED is represented by a first-order Laguerre-Gaussian beam (Fig. 5b top):

$$
\begin{aligned}
I_{\text {exc }}(r) & =I_{0} \exp \left(-\frac{2 r^{2}}{w_{0}^{2}}\right) \\
I_{S T E D}(r) & =I_{0}\left(\frac{2 r^{2}}{w_{0}^{2}}+\zeta\right) \exp \left(1-\frac{2 r^{2}}{w_{0}^{2}}\right)
\end{aligned}
$$

where $w_{0}$ is the beam width (excitation $w_{0}=191 \mathrm{~nm}$ corresponding to FWHM=225nm, STED $w_{0}=285 \mathrm{~nm}$ and FWHM=190 nm $1660 \mathrm{~nm}$ for inner/outer doughnut diameter respectively), $\zeta$ is the residual relative STED intensity at the targeted coordinate (in the minimum of the doughnut beam). Confocal detection is again defined as $1 \mathrm{AD}$ at the excitation wavelength, corresponding to the final confocal diffraction-limited lateral resolution $\Delta r_{\text {conf }}=\frac{\Delta r}{\sqrt{2}} \approx 162 \mathrm{~nm}$. Most parameters are fixed during simulation. The STED pulse properties are varied (duration $\tau$, delay $t$, power $P_{S T E D}$ ). After applying the beams' spatial distributions in the model, we obtained the fluorescence profile and probability of photobleaching as a function of lateral position (Fig. 5b bottom). The resolution is defined as the FWHM of the fluorescence profile. The photobleaching value represents an integral over the spatial distribution of photobleaching probability (without confocal detection).

\subsection{Resolution scaling with STED power}

We modelled the resolution scaling of a STED nanoscope as a function of STED average power $P_{S T E D}$. As shown previously ${ }^{6}$, the resolution follows a square-root law:

$$
\Delta r=\frac{\Delta r_{\text {conf }}}{\sqrt{1+P_{S T E D} / P_{\text {Sat }}}}
$$

where $\Delta r_{\text {conf }}=162 \mathrm{~nm}, P_{S T E D}$ is the average STED power, $P_{S a t}$ is a characteristic power to guarantee off-switching. We fit the formula (with a single free parameter $P_{S a t}$ ) to the modelled resolution scaling, finding excellent agreement with the expected behaviour (Supplementary Fig. S7a, $P_{\text {Sat }}=23 \mathrm{~mW}$; STED pulse duration $\tau=200 \mathrm{ps}$, STED delay $t=150 \mathrm{ps}$, residual relative STED intensity at doughnut minimum $\zeta=0$ ).

\subsection{Optimal delay between excitation and STED pulse}

The temporal distribution of de-excitation photons after the ultrafast excitation has an influence on the image quality. To enable a fair comparison between different STED pulse durations $\tau$, we chose a favourable time delay $t$ between the excitation and the STED pulse for non-gated detection (Supplementary Fig. S7b, circles) and gated detection (Supplementary Fig. S7b, dashed lines, starting at $t_{\text {gate }}=t+\frac{1}{2} \tau$ ). Taking into account the fluorescence decay, the flux of STED photons should be the highest just after the excitation, when many molecules relax by spontaneous emission. However, a simple overlap of the excitation and STED Gaussian pulses' maxima results in loss of half of the de-excitation photons: they do not contribute to depopulation of the excited state and, moreover, may interact with molecules in unwanted ways, causing excitation by the STED-light photons and photobleaching. On the other hand, a significant delay between pulses causes a reduction of the de-excitation efficiency because the STED intensity (and thus stimulated emission probability) is not high enough at the beginning of the fluorescence decay to compete with the spontaneous relaxation. This results in the early fluorescence component in the registered superresolution signal, creating wings (a halo) in the effective PSF $^{7}$. To characterize the optimal conditions, we modelled the resolution as a function of delay $t$ between excitation and STED pulse for different STED pulse durations $\tau$ (Supplementary Fig. S7b). The resolution changes as a function of delay $t$ are highest for a low average STED power ( $\sim$ few $\mathrm{mW}$ at $80 \mathrm{MHz}$ repetition rate), as the resolution curve is very steep in this regime (Supplementary Fig. S7a). To highlight the changes, we modelled the resolution for rather low constant time-averaged STED power $P_{S T E D}=10 \mathrm{~mW}$. The optimal delay (also for higher STED powers) depends on the pulse duration and is in the range $\left(\frac{1}{2} \mathrm{FWHM}\right.$; FWHM) of the STED pulse duration $\tau$. We therefore selected the delay $t=0.75 \tau$ for all investigated Gaussian pulses. The best resolution can be obtained for the shortest pulses $(\tau \approx 10 \mathrm{ps})$. Applying much longer pulses $(\sim 1 \mathrm{~ns})$ reduces the achievable resolution due to early fluorescence ${ }^{7}$. This can be mitigated by applying gating, with the gating time equal to approximately the STED pulse duration: $t_{\text {gate }} \approx \tau$. In this case, the resolution is nearly independent of the pulse duration. The downside is a reduced fluorescence signal. 


\subsection{Bleaching at different STED powers}

Similarly to the experimental results, our simulations reveal a sublinear scaling of bleaching with average STED power for the experimentally investigated power range (Supplementary Fig. S7c, left). Occupancy of the excited state (which largely initializes all bleaching pathways) is quite efficiently counter-acted by the STED light. In this regime, photobleaching may even be reduced in comparison to confocal microscopy measurements, because efficient transfer of the excited molecules to the ground state prevents them from following one of the possible destructive pathways. However, at higher average powers, the dependence of photobleaching should exhibit a superlinear scaling (Supplementary Fig. S7c, right). The reason is that, starting at higher STED pulse energies, the one-photon absorption events of STED light become equally significant as the absorption of photons from the excitation pulse. Thus, photobleaching initiated by STED-light-induced excitation of fluorophores becomes relevant, resulting in an increased region of photobleaching and a higher order of photobleaching scaling. A high probability of stimulated emission, and the typically confocal detection scheme employed in measurements, render the importance of STEDbeam excitation events typically largely invisible in the detected fluorescence signal. As an example, for ATTO647N, the STED-light-induced fluorescence is $<0.5 \%$ relative to the signal by excitation, at the average STED power $P_{S T E D}=1000 \mathrm{~mW}$ ( $\tau=1000 \mathrm{ps}$ ). However, the majority of bleaching is initiated by linear absorption of STED-light photons, and a reduction of the linear absorption coefficient $\left(\sigma_{1 P E}\right)$ by half reduces bleaching nearly by half (data not shown). Since the effects of unwanted one-photon absorption depend on the pulse energy rather than the temporal distribution of photons, this limits the STED pulse energies that can be applied (and thus resolution) before the fluorescent marker is photobleached. To reduce photobleaching, it is necessary to minimize the one-photon absorption coefficient by shifting the STED wavelength to longer wavelengths or changing the fluorescent dye to ones with bluer absorption spectra. For example, shifting the wavelength from $750 \mathrm{~nm}$ to $770 \mathrm{~nm}$ for ATTO647N should reduce the linear absorption cross-section approximately by an order of magnitude ( $\sim$ ten times), while the stimulated emission cross-section is reduced only by half.

a Resolution scaling with STED power

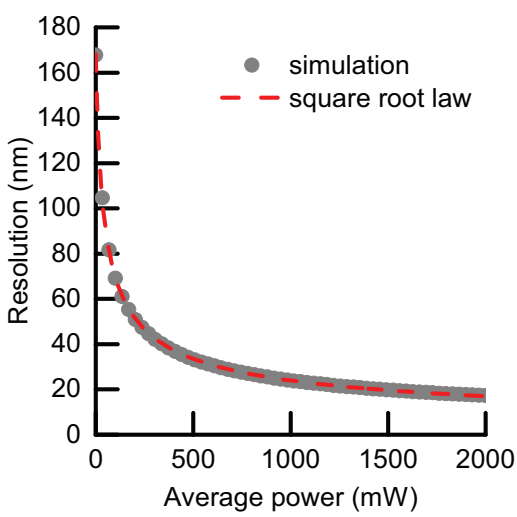

b Optimal delay between excitation and STED pulses

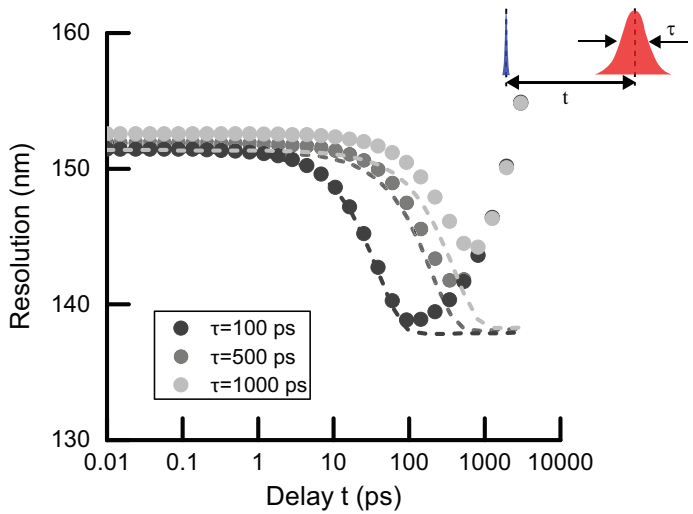

C Photobleaching scaling with average STED power ( $\tau=1 \mathrm{~ns})$
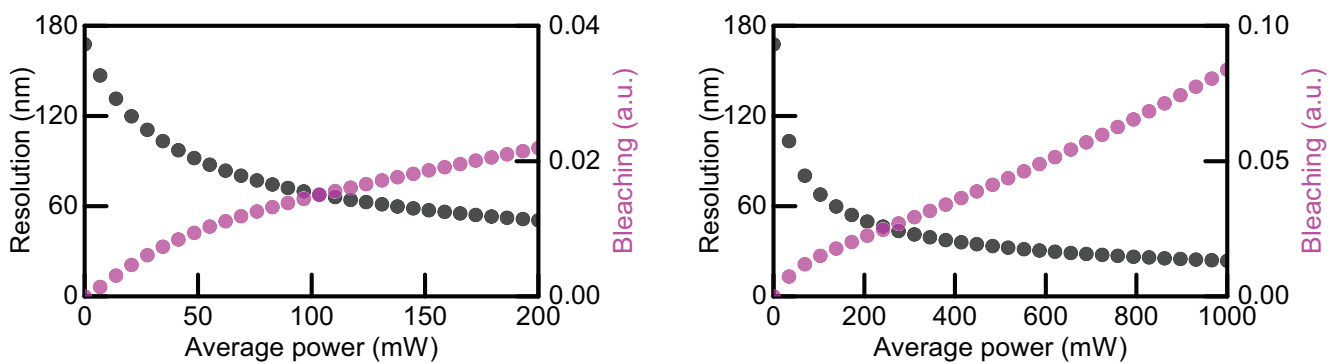

Supplementary Figure S7. Simulation of resolution and photobleaching for ATTO647N. (a) Resolution scaling with average STED power for STED pulse duration $\tau=200 \mathrm{ps}$. The dashed line represents the square-root function with a single free parameter $\left(P_{S a t}=23 \mathrm{~mW}\right)$. (b) Optimal time delay $t$ between ultrafast excitation and STED pulse for different STED pulse durations $\tau\left(P_{S T E D}=10 \mathrm{~mW}\right)$. The dashed lines corespond to gated detection, with $t_{\text {gate }}=t+\frac{1}{2} \tau$. (c) Photobleaching and resolution vs. time-averaged STED power for moderate deexcitation powers (left, negligible linear absorption at STED wavelength) and higher powers (right). STED pulse duration $\tau=1 \mathrm{~ns}$, repetition rate $f=80 \mathrm{MHz}$. 


\section{Simulation of de-excitation, bleaching and STED-light-induced fluorescence for ATT0590}

The presented photobleaching model gives a good description if photobleaching follows a single intensity scaling. For the majority of the dyes (ATTO590, STAR580, STAR635P) the bleaching mechanism is more complicated (see Fig. 3b), indicating different dominant mechanisms for different peak intensity ranges. As, in general, photobleaching is associated with singlet and triplet ladders of states, the details of which were not accessible in our experiments, it was not possible to include all relevant parameters in the numerical modelling. A first obstacle is the much longer time scales for triplet-related processes (with lifetime of the first excited triplet state micro- to milliseconds) compared to singlet processes (with nanosecond lifetime of the first excited singlet state). A second obstacle lies in the significant increases in the numbers of free parameters, which are typically not directly measurable and often depend on the environment of the dye (such as ISC rate(s), triplet state lifetimes and associated photobleaching rates). Nonetheless, we modelled the behaviour of ATTO590 in STED microscopy, following the same procedure as described above (Section 4). Since the photobleaching differs for different STED intensity regimes, the photobleaching rate from the excited state $S_{1}$ in our model must reflect two of these regimes and can be approximated by:

$$
k\left(I_{S T E D}\right)=k_{0} \cdot\left(\frac{I_{S T E D}}{1 W / m^{2}}\right)+k_{1} \cdot\left(\frac{I_{S T E D}}{1 W / m^{2}}\right)^{1.9} .
$$

The orders of nonlinearity were extracted from experimental data (see Fig. $3 \mathrm{~b}$, Table 1 ). The bleaching rates $k_{0}, k_{1}$ as well as the probabilities of stimulated emission $\sigma_{S T E D}$ and STED light absorption by one- and two-photon processes $\sigma_{1 P E}, \sigma_{2 P E}$ were found by fitting the model curves to the experimental data (see Supplementary Fig. S8a). All varied parameters are listed in Supplementary Table S4. As estimated based on normalized spectra, the stimulated emission probability is slightly lower than for ATTO647N, since the applied STED wavelength is further from the emission maximum of the dye (Fig. 1b). The probability of single-photon absorption is also lower since the STED wavelength is shifted from the absorption maximum. At the same time, ATTO590 is characterized by a significantly higher two-photon absorption probability. The experimental data and modelled curves are presented in Supplementary Fig. S8a,b). The presented simple numerical modelling only roughly describes the performance of ATTO590 and other markers in STED microscopy, as seen also from moderate but noticeable deviations in the power dependence of bleaching already for STED powers on the order of $100 \mathrm{~mW}$ in Supplementary Fig. S8b. This advises caution in the extrapolation to higher average STED powers and quantitative predictions of performance in the highest-resolution regime. The resolution and bleaching scaling for different STED pulse durations are presented in Supplementary Fig. S8c,d. ATTO590 shows slightly lower resolution than ATTO647N for the same average power. The bleaching has a stronger nonlinear component, however this difference manifests only at high STED peak powers (short pulses, compare Fig. 5c). With increasing STED pulse duration, the bleaching decreases to similar values as for ATTO647N. The resolution and bleaching scalings with STED time-averaged power are presented in Supplementary Fig. S8e,f. The STED pulse duration for the modelling equalled $\tau=1000 \mathrm{ps}$. The higher-order scaling of bleaching compared to ATTO647N suggests that further increases of pulse duration may indeed be beneficial for this dye. It is important to note that the spatial distribution of photobleaching is significantly different than for ATTO647N (compare Fig. 5f). Despite the different relative prominence of the involved optical transitions, the overall performance of the two dyes in STED microscopy is comparable.

Supplementary Table S4. Parameters extracted from experimental data for modelling ATTO590 behaviour in STED nanoscopy. All other parameters were fixed (see Supplementary Table S3). nRMSE is normalized root mean square error of the fit. The modelled curves and experimental data are presented in Supplementary Fig. S8a.

\begin{tabular}{lccc}
\hline & Temporal domain & & \\
Parameter & Symbol & Value & nRMSE \\
\hline stimulated emission cross-section & $\sigma_{S T E D}$ & $4 \times 10^{-18} \mathrm{~cm}^{2}$ & $4 \%$ \\
STED one-photon excitation & $\sigma_{1 P E}$ & $8 \times 10^{-22} \mathrm{~cm}^{2}$ & $17 \%$ \\
STED two-photon excitation & $\sigma_{2 P E}$ & $352 \mathrm{GM}$ & \\
photobleaching rate & $k_{0}(b=1)$ & $2.5 \times 10^{-5} \mathrm{~Hz}$ & $5 \%$ \\
photobleaching rate & $k_{1}(b=1.9)$ & $9 \times 10^{-18} \mathrm{~Hz}$ & \\
\hline
\end{tabular}



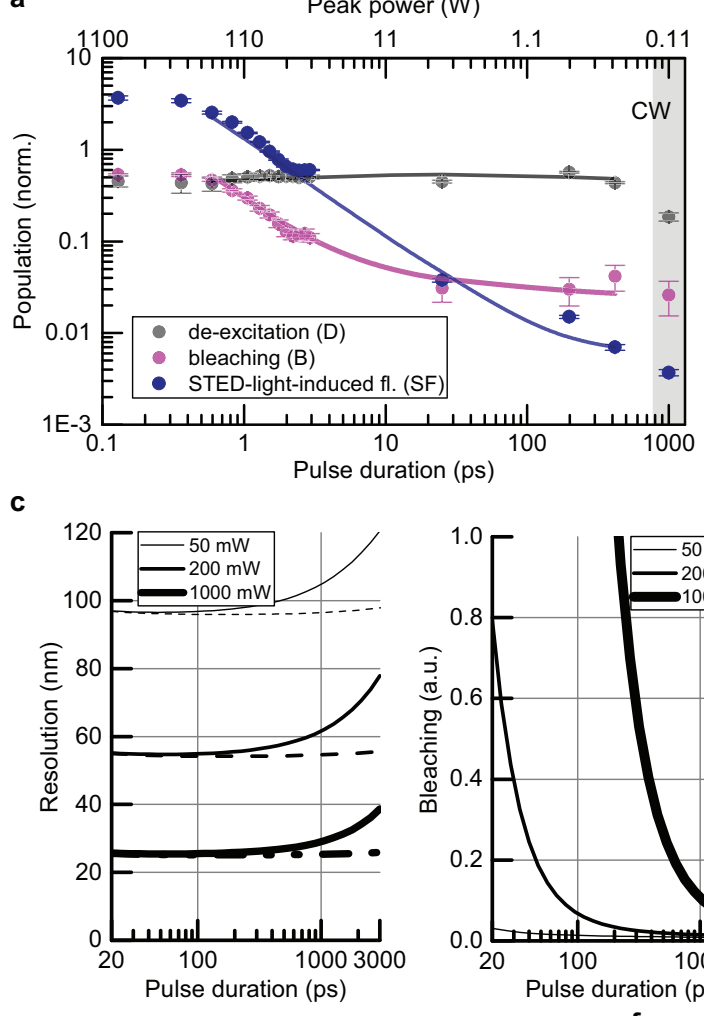

e
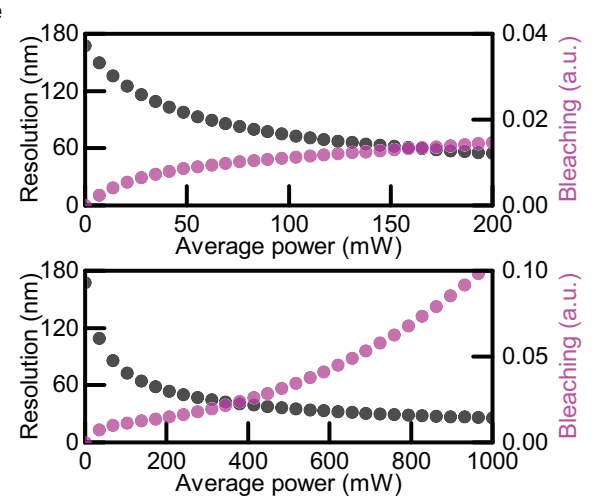
f b

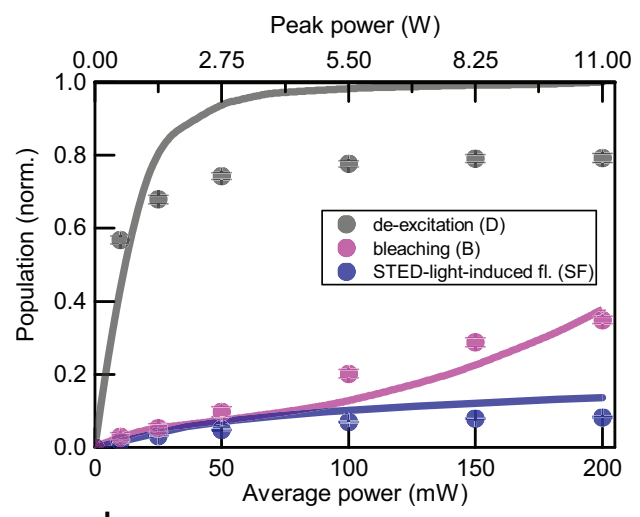

d

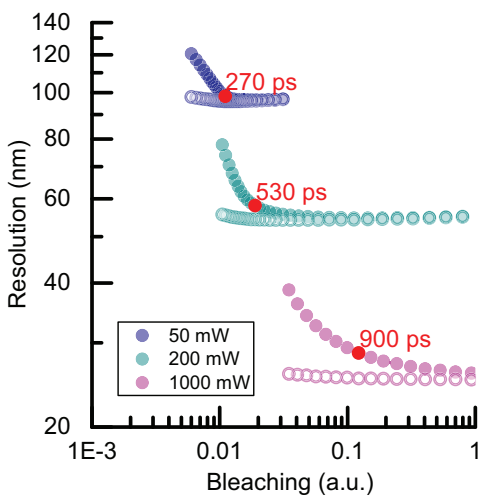

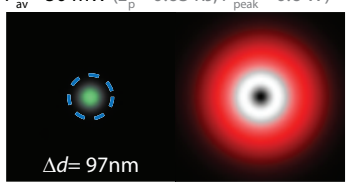

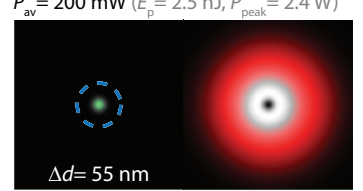

$P_{\mathrm{av}}=500 \mathrm{~mW}\left(E_{\mathrm{p}}=6.3 \mathrm{n} J, P_{\text {peak }}=5.9 \mathrm{~W}\right)$
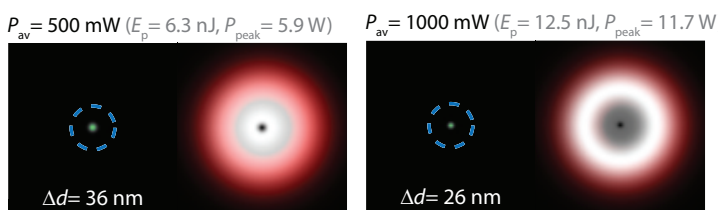

Supplementary Figure S8. Modelling ATTO590 in STED microscopy. (a) De-excitation, bleaching and STED-light-induced fluorescence vs. pulse duration for ATTO590. CW represents measurements for continuous-wave laser operation. The solid lines represent values calculated within the photobleaching model (see Supplementary Table S4). Time-averaged STED power: $10 \mathrm{~mW}\left(E_{p}=0.125 \mathrm{~nJ}\right)$ measured at the back aperture of the objective lens. (b) Comparison between experimental data and modelling for different time-averaged STED powers. STED pulse duration: $\tau=200 \mathrm{ps}$. (c) Resolution and bleaching as a function of pulse duration for three time-averaged STED powers. The dashed lines represent gated detection starting at $t_{\text {gate }}=\tau$. (d) The same data as in $\mathrm{c}$ presented as resolution vs. bleaching. The lowest bleaching and the highest resolution can be obtained by selecting the STED pulse duration and applying gated detection. (e) Photobleaching and resolution vs. time-averaged STED power for moderate de-excitation powers (top) and higher powers (bottom). STED pulse duration $\tau=1 \mathrm{~ns}$. (f) Spatial distribution of fluorescence (green), normalized probabilities of photobleaching (linear grey scale, with highest photobleaching in white) and normalized STED beam profile as reference (red) for different average STED powers (STED pulse duration: $\tau=1000 \mathrm{ps}$ ). The stated resolution values $\Delta d$ represent the respective FWHM of the fluorescence profile. The dashed blue circles correspond to the FWHM of the excitation focal spot. 
Supplementary Table S5. Different implementations of STED microscopy with red dyes and fluorescent beads. *unpublished own measurements

\begin{tabular}{|c|c|c|c|c|c|c|c|c|c|}
\hline$\#$ & Dye & $\lambda_{\text {STED }}(\mathrm{nm})$ & $\tau(\mathrm{ns})$ & $f(\mathrm{MHz})$ & $P_{S T E D}(\mathrm{~mW})$ & $E_{p}(\mathrm{~nJ})$ & $P_{S T E D}{ }_{\text {peak }}(\mathrm{W})$ & $\Delta r(\mathrm{~nm})$ & Ref. \\
\hline 1 & KK114 & 775 & 1 & 20 & 166 & 8.3 & 6.5 & 30 & 8 \\
\hline 2 & STAR635P & 775 & 1.2 & 20 & 200 & 10 & 7.83 & 20 & 9 \\
\hline 3 & $\begin{array}{l}\text { ATTO647N } \\
\text { STAR635P }\end{array}$ & 775 & 1 & 20 & 200 & 10 & 7.83 & 25 & 10 \\
\hline 4 & $\begin{array}{l}\text { Crimson } \\
\text { beads }\end{array}$ & 750 & 0.2 & 80 & 300 & 3.75 & 17.6 & 40 & $*$ \\
\hline 5 & ATTO647N & 750 & $\mathrm{CW}$ & - & 423 & 5.3 & 0.42 & 50 & 11 \\
\hline 6 & ATTO633 & 750 & 0.2 & 76 & 114 & 1.5 & 7 & 40 & 12 \\
\hline 7 & ATTO633 & 735 & 0.09 & 1 & 2.4 & 2.4 & 25 & 40 & 13 \\
\hline 8 & $\begin{array}{l}\text { fluorescent } \\
\text { beads }\end{array}$ & 763 & $\mathrm{CW}$ & - & 516 & 6.45 & 0.52 & 71 & 14 \\
\hline 9 & ATTO647N & 770 & 0.25 & 80 & 168 & 2.1 & 7.89 & 85 & 15 \\
\hline 10 & $\begin{array}{l}\text { Crimson } \\
\text { beads }\end{array}$ & 735 & 0.2 & 76 & 375 & 8.95 & 28 & 25 & 6 \\
\hline 11 & ATTO647N & 760 & 0.2 & 76 & 70 & 0.921 & 4.33 & 89 & 16 \\
\hline 12 & ATTO647N & 760 & 0.2 & 80 & 130 & 1.62 & 7.63 & 78 & 17 \\
\hline 13 & $\begin{array}{l}\text { Crimson } \\
\text { beads }\end{array}$ & 730 & $\mathrm{CW}$ & - & 1250 & 15.625 & 1.25 & 34 & 11 \\
\hline
\end{tabular}

\section{Comparison: Resolution in STED microscopy reported for red dyes and fluorescent beads}

The resolution improvement in STED microscopy relies on delivering to the sample a certain number of de-excitation photons, which force fluorophores to remain dark during signal detection in a spatially selective way. For de-excitation pulses shorter than the excited-state lifetime, the number of delivered photons (i.e., the pulse energy) roughly determines the resolution improvement (Supplementary Fig. S9a). For example, to obtain $25 \mathrm{~nm}$ resolution one needs to apply $\sim 11 \mathrm{~nJ}$ pulses (back aperture), for both $1 \mathrm{~ns}$ and 200 ps STED pulses. These energies correspond to substantially different peak powers. In the first case, the peak power is $\sim 7 \mathrm{~W}$. Such peak powers were successfully implemented in STED microscopy before, for crimson beads as well as the fluorescent dye ATTO633 (e.g. points 4, 6, 7, 10). However, to deliver the same energy in 200 ps STED pulses would require a peak power of $\sim 50 \mathrm{~W}$, well above what has been reported so far (Supplementary Fig. S9b). Reducing the peak power by implementing long STED pulses directly diminishes the nonlinear component of photobleaching for fluorescent dyes.

It is important to note that the overall molecular photobleaching of fluorophores can be divided into two categories: low-order photobleaching and high-order photobleaching. In the first case, the photobleaching probability of the excited molecule is independent or linear with the applied light intensity. One possible pathway in this category is the photobleaching associated with the first triplet state. The magnitude of photobleaching in this case depends on the internal properties of the dye and its microenvironment (reactivity, intersystem crossing ISC) and the lifetime of the excited states (both singlet and triplet). For this bleaching mechanism (dominant in low-power applications, and especially important for blue-orange fluorescent dyes with high ISC), the optimal strategy is to reduce triplet build-up (by T-Rex, quenchers) and to push the excited molecules to the non-reactive ground state as soon as possible (short de-excitation pulses). In this case, application of STED pulses can even reduce photobleaching in confocal microscopy, due to a reduction of the effective time which molecules spend in the excited state. The other mechanism, high-order photobleaching, is associated with higher excited states (singlets and triplets) of the fluorophore, which can be reached by absorption of photons. As a result, the effective photobleaching rate from the excited state depends on the STED-light (photon flux) with order $b>1$. High-order photobleaching can be reduced by applying long de-excitation pulses up to approximately the excited-state lifetime of the fluorescent dye. Both processes contribute to the photodestruction of the fluorophore. For different markers and different environments, the major mechanism(s) may change, and further studies are necessary depending on the imaging context.

This study shows that, for ATTO647N, the scaling of photobleaching is nonlinear for all intensities investigated $(b=1.4)$. This single bleaching scaling may be explained by its low ISC and thus low-order photobleaching, which is not limiting the performance of this dye in STED microscopy. For all other dyes, we report a nonlinear behaviour for the peak powers ( $>11 \mathrm{~W}$ ) currently applied in STED microscopy to reach the best resolutions. 

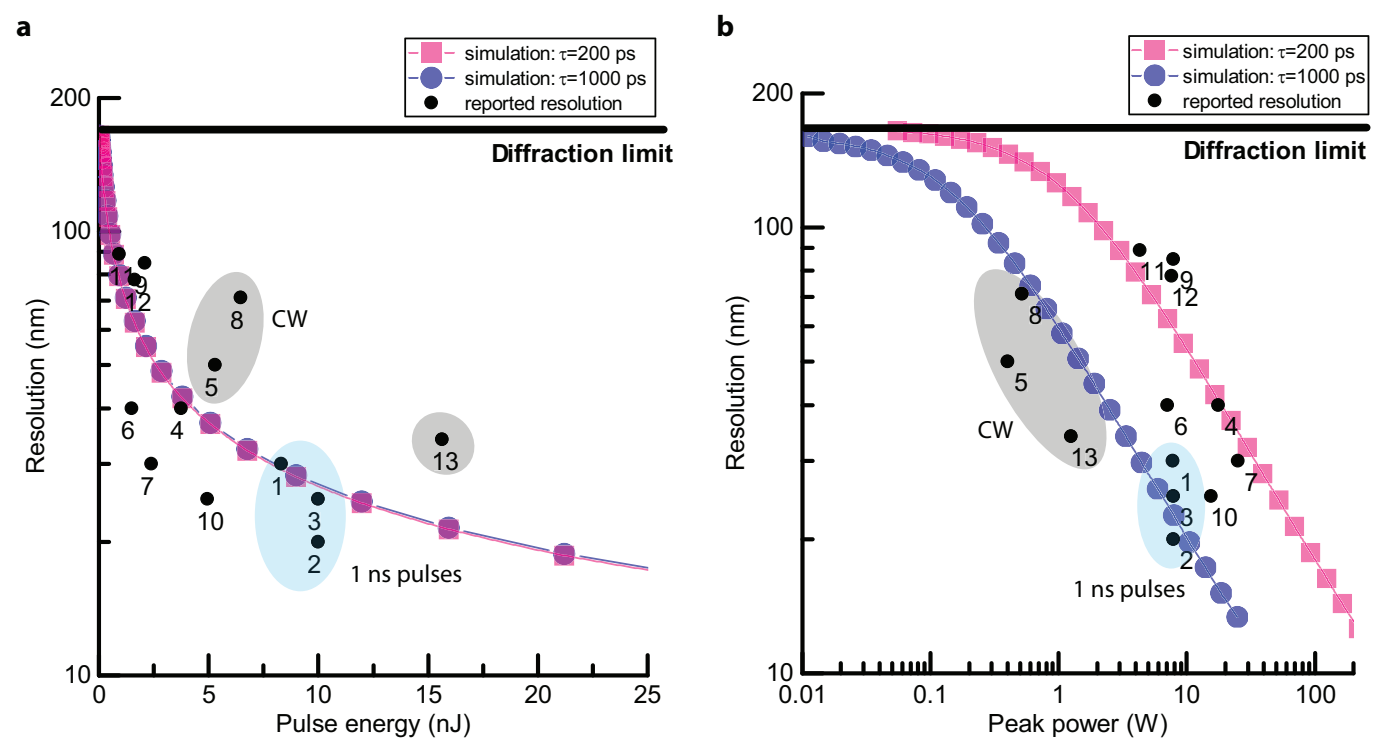

Supplementary Figure S9. Resolution reported for different STED implementations as a function of pulse energy (a) and pulse peak power (b) for different red dyes and crimson beads (see Supplementary Table S5). Pulse energies and peak powers were calculated at the back aperture of the objective lens. The data shown in colour represents simulation results for ATTO647N, for 200 ps (squares) and $1 \mathrm{~ns}$ (circles) Gaussian STED pulses at $80 \mathrm{MHz}$ repetition rate. The top black line indicates the diffraction-limited confocal resolution for an excitation wavelength of $635 \mathrm{~nm}$.

\section{Parameter calculations}

\section{Time-averaged power at back aperture of the objective lens}

All time-averaged STED powers were estimated at the back aperture of the objective lens. Since some publications specified this value in the focal plane, the powers at the back aperture were calculated as:

$$
P_{\text {back }}=T^{-1} P_{\text {focal }}
$$

where $P_{b a c k}$ is the average power at the back aperture, $P_{\text {focal }}$ is the power in the focal plane, and $T$ relates both measurements (and includes the transmission of the objective lens). $T$ was calculated based on publications which stated both values ${ }^{9,10}$ to $T=0.6$.

\section{Supplementary References}

1. Wong, V. \& Walmsley, I. A. Analysis of ultrashort pulse-shape measurement using linear interferometers. Opt. Lett. 19, 287-289 (1994).

2. Hacker, M., Stobrawa, G. \& Feurer, T. Iterative Fourier transform algorithm for phase-only pulse shaping. Opt. Express 9, 191-199 (2001).

3. Cerullo, G., Bardeen, C., Wang, Q. \& Shank, C. High-power femtosecond chirped pulse excitation of molecules in solution. Chem. Phys. Lett. 262, $362-368$ (1996).

4. Cao, J., Bardeen, C. J. \& Wilson, K. R. Molecular " $\pi$ pulse” for total inversion of electronic state population. Phys. Rev. Lett. 80, 1406-1409 (1998).

5. Velasco, M. G. M., Allgeyer, E. S., Yuan, P., Grutzendler, J. \& Bewersdorf, J. Absolute two-photon excitation spectra of red and far-red fluorescent probes. Opt. Lett. 40, 4915-4918 (2015).

6. Harke, B. et al. Resolution scaling in STED microscopy. Opt. Express 16, 4154-4162 (2008).

7. Moffitt, J. R., Osseforth, C. \& Michaelis, J. Time-gating improves the spatial resolution of STED microscopy. Opt. Express 19, 4242-4254 (2011).

8. Schmidt, R. et al. Mitochondrial cristae revealed with focused light. Nano Lett. 9, 2508-2510 (2009). 
9. Göttfert, F. et al. Coaligned dual-channel STED nanoscopy and molecular diffusion analysis at 20 nm resolution. Biophys. J. 105, L01 - L03 (2013).

10. Wurm, C. A. et al. Novel red fluorophores with superior performance in STED microscopy. Opt. Nanoscopy 1, 7 (2012).

11. Willig, K. I., Harke, B., Medda, R. \& Hell, S. W. STED microscopy with continuous wave beams. Nat. Methods 4, 915-918 (2007).

12. Punge, A. et al. 3D reconstruction of high-resolution STED microscope images. Microsc. Res. Tech. 71, 644-650 (2008).

13. Wildanger, D., Rittweger, E., Kastrup, L. \& Hell, S. W. STED microscopy with a supercontinuum laser source. Opt. Express 16, 9614-9621 (2008).

14. Liu, Y. et al. Achieving $\lambda / 10$ resolution CW STED nanoscopy with a Ti:Sapphire oscillator. PLoS ONE 7, e40003 (2012).

15. Bianchini, P., Harke, B., Galiani, S., Vicidomini, G. \& Diaspro, A. Single-wavelength two-photon excitation-stimulated emission depletion (SW2PE-STED) superresolution imaging. Proc. Natl. Acad. Sci. USA 109, 6390-6393 (2012).

16. Vicidomini, G. et al. STED nanoscopy with time-gated detection: Theoretical and experimental aspects. PLoS ONE 8, e54421 (2013).

17. Pellett, P. A. et al. Two-color STED microscopy in living cells. Biomed. Opt. Express 2, 2364 (2011). 
Chapter 5

Ground state depletion nanoscopy resolves semiconductor nanowire barcode segments at room temperature 



\title{
Ground State Depletion Nanoscopy Resolves Semiconductor Nanowire Barcode Segments at Room Temperature
}

\author{
Joanna Oracz, ${ }^{*}, \dagger$ Karl Adolfsson, ${ }^{\S}$ Volker Westphal, ${ }^{\dagger}$ Czesław Radzewicz, ${ }^{\ddagger}$ Magnus T. Borgström, ${ }^{\S}$
} Steffen J. Sahl, ${ }^{\dagger}$ Christelle N. Prinz, ${ }^{*}, \S$ and Stefan W. Hell ${ }^{*}{ }^{\dagger} \odot$

${ }^{\dagger}$ Department of NanoBiophotonics, Max Planck Institute for Biophysical Chemistry, 37077 Göttingen, Germany

${ }^{\ddagger}$ Faculty of Physics, University of Warsaw, 02-093 Warsaw, Poland

${ }^{\S}$ Division of Solid State Physics and NanoLund, Lund University, 22100 Lund, Sweden

\section{Supporting Information}

ABSTRACT: Nanowires hold great promise as tools for probing and interacting with various molecular and biological systems. Their unique geometrical properties (typically $<100$ $\mathrm{nm}$ in diameter and a few micrometers in length) enable minimally invasive interactions with living cells, so that electrical signals or forces can be monitored. All such experiments require in situ high-resolution imaging to provide context. While there is a clear need to extend visualization capabilities to the nanoscale, no suitable super-resolution far-
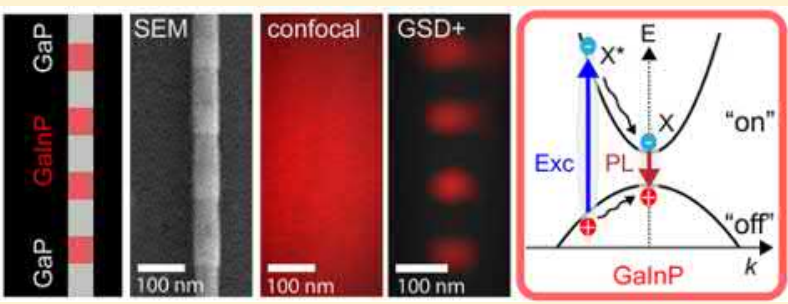

field photoluminescence microscopy of extended semiconductor emitters has been described. Here, we report that ground state depletion (GSD) nanoscopy resolves heterostructured semiconductor nanowires formed by alternating $\mathrm{GaP} / \mathrm{GaInP}$ segments ("barcodes") at a 5-fold resolution enhancement over confocal imaging. We quantify the resolution and contrast dependence on the dimensions of GaInP photoluminescence segments and illustrate the effects by imaging different nanowire barcode geometries. The far-red excitation wavelength $(\sim 700 \mathrm{~nm})$ and low excitation power $(\sim 3 \mathrm{~mW})$ make GSD nanoscopy attractive for imaging semiconductor structures in biological applications.

KEYWORDS: Nanowires, microscopy, super-resolution imaging, photoluminescence, semiconductor heterostructures

$\mathrm{H}$ igh aspect ratio nanostructures such as nanowires (NWs) or nanotubes can be utilized as sensors or needles to measure and perturb the surface or interior of a cell. ${ }^{1-3}$ These structures, owing to their geometry and optoelectronic properties, are candidates for new-generation nanometer-sized probes for biological molecules, including enzyme or other protein recognition, ${ }^{4-6}$ analysis of electrical activity, ${ }^{7,8}$ longterm molecular tracking, ${ }^{9}$ intracellular delivery, ${ }^{10,11}$ cellular force measurements, ${ }^{12}$ or nerve tissue engineering. ${ }^{13,14}$ All of these applications are to date still hampered by our relatively poor understanding of how extrinsic materials and their topology influence cell properties. ${ }^{15,16}$

To fully exploit the potential of one-dimensional nanostructures in biological research, it is necessary to establish minimally invasive, simple optical methods that allow controlled measurements of their interactions with cells. The high degree of control over NW synthesis enables the fine-tuning of their geometrical and optoelectronic properties, which makes NWs perfect model structures in this respect. Several studies have examined the interactions of NWs with cells, mainly using confocal microscopy. NWs have been indirectly visualized as dark spots due to spatial exclusion within labeled cells ${ }^{17,18}$ or were directly labeled with fluorescent organic dyes. ${ }^{19-21}$ To circumvent bleaching issues associated with these methods, it has been recently proposed ${ }^{22}$ to use the intrinsic photo- luminescence of NW heterostructures as contrast method for their visualization in biological systems. ${ }^{22,23}$ However, the diffraction-limited resolution of conventional lens-based microscopy restricts the amount of information that can be extracted from such images. While imaging at higher resolution, scanning electron microscopy (SEM) only provides surface information, and reduces throughput in the case of slice-andview SEM techniques. Therefore, there is a need for methods allowing for optical imaging of photoluminescent nanowires at resolution below the diffraction limit.

Addressing the resolution problem, a variety of subdiffraction far-field microscopy (here in short, "nanoscopy") methods have been reported for organic fluorophores, photoswitchable fluorescent proteins, quantum dots, and color centers in diamond. Breaking the far-field diffraction barrier ultimately relies on judiciously exploiting intrinsic emitter properties and transferring emitters between (at least) two states (typically signaling "on" and non-signaling "off") to achieve their separation $^{24,25}$ at subdiffraction length scales. In far-field nanoscopy, the properties of the accessible intrinsic states determine the best choice of imaging approach and its

Received: February 3, 2017

Revised: March 3, 2017

Published: March 6, 2017 
(a)

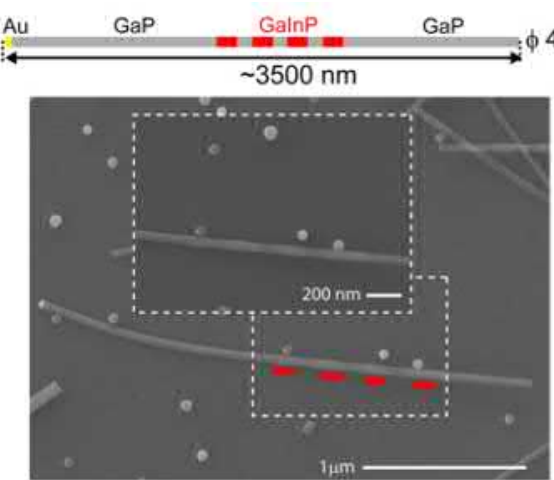

(c)

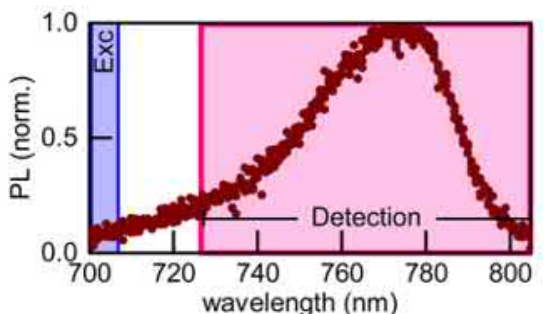

(b)

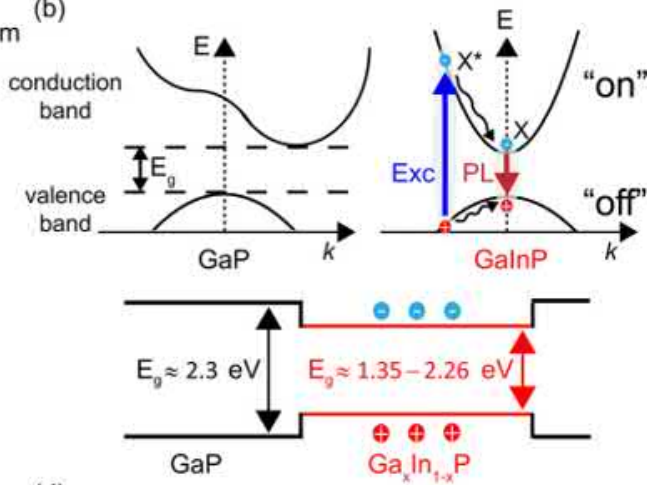

(d)

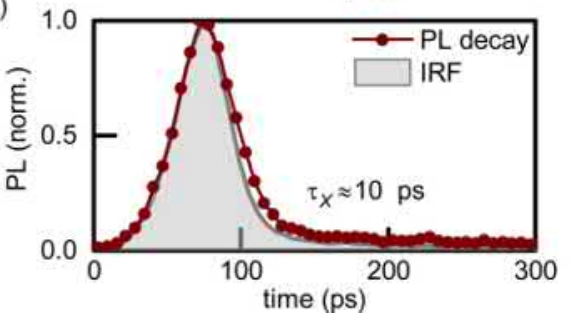

Figure 1. Properties of heterostructured nanowires. (a) Schematic drawing of a barcode nanowire and SEM image. Red lines indicate the photoluminescent GaInP segments. (b) Band structure diagram for GaP and GaInP. (c) Photoluminescence spectrum of a single NW GaInP segment. (d) Photoluminescence lifetime measured at room temperature with instrument response function (IRF, $\tau_{\text {jitter }}=40 \mathrm{ps}$ ) shown in gray. The estimated photoluminescence lifetime after deconvolution was $\tau_{\mathrm{X}} \approx 10 \mathrm{ps}$.

parameters. The emitter characteristics (such as quantum yield, optical cross sections, intersystem crossing, and photostability) affect the imaging performance and ultimately determine the attainable resolution.

We sought to explore the intrinsic photoluminescence (PL) of nanowire axial heterostructures with the goal of improving the resolution for this class of emitter. The nanowires consisted of alternating non-luminescent gallium phosphide $(\mathrm{GaP})$ and luminescent gallium indium phosphide (GaInP) segments ("barcodes") (Figure 1a). They were synthesized by metal organic vapor phase epitaxy (MOVPE) growth, similarly to previous experiments. ${ }^{22}$ The details of the synthesis are described in the Supporting Information. In previous experiments, no emission from $\mathrm{GaP}$ was observed, which can be explained by the fact that, typically, $\mathrm{GaP}$ has an indirect bandgap $(\sim 2.3 \mathrm{eV})$. A $\mathrm{Ga}_{x} \mathrm{In}_{1-x} \mathrm{P}$ alloy has a direct and tunable bandgap over a wide range of compositions $(\sim 1.35-2.26 \mathrm{eV})$, which can be controlled during the growth procedure. Upon illumination, valence band electrons are excited to the conduction band by any photons with energy greater than the bandgap energy, and recombination of electron-hole pairs (excitons) results in bright, stable, and spectrally broad PL at room temperature (Figure 1b,c). The detected emission has typically a lifetime of $\tau_{X} \sim 10-100$ ps (Figure 1d).

Most popular far-field nanoscopy methods are not readily applicable to semiconductor nanowires. The coordinatestochastic approaches (e.g., PALM ${ }^{26,27} / \mathrm{STORM}^{28}$ ), which rely on temporal signal intermittencies (blinking) of individual emitters, cannot be applied to the NWs due to the constant background PL contributions from many excitons within an extended segment. Alternatively, inhibiting short-lifetime photoluminescence by a coordinate-targeted strategy like STED $^{29}$ is challenged by unwanted absorption of the deexcitation light. Thus, as NWs feature high photostability, a different strategy appeared promising: to control the transfer of electrons to the conduction band ("on" state, Figure 1b) in a coordinate-targeted manner, transferring electrons out of the valence band "off" state, as originally suggested in the classic ground state depletion (GSD) concept ${ }^{30-32}$ and later also used in saturated structured illumination microscopy (SSIM). ${ }^{33,34}$ The majority of carriers are thus forced to reside most of the time in the "on" state by using an excitation intensity distribution in the focal plane featuring one or even many zeros. Upon scanning, this leads to the acquisition of a "negative" image with subdiffraction resolution. Excitation and photoluminescence are ideally disallowed at the zero(s), that is, the targeted coordinate(s). Everywhere else, the transition out of the ground state is guaranteed, and signal is generated.

Designating the two electron states as "on" and "off", electron transfer to the "on" state and thus photoluminescence can be virtually guaranteed for excitation intensities $I \gg$ $\frac{h \nu}{\tau_{X} \sigma_{\mathrm{exc}}} \approx I_{\mathrm{s}}$, where $h \nu$ is the photon energy, $\sigma_{\text {exc }}$ is the excitation cross-section, $\tau_{X}$ is the photoluminescence lifetime and $I_{s}$ denotes a characteristic threshold intensity at which half of the maximal PL signal $\eta$ is elicited. The estimated fraction of electrons in the "on" state scales with $I$ as follows: $\eta \approx 1 /\left(1+\frac{I_{s}}{I}\right)$. The effective point spread function (PSF), which governs resolution, can therefore be described by $h_{\text {eff }}(x) \approx \frac{1}{1+\frac{I_{s}}{I(x)}}$, where $I(x)$ is the spatial distribution of a doughnut-shaped excitation beam, which can be approximated around the targeted coordinate as $I(x) \approx I_{\max }\left(\frac{x^{2}}{\Delta d^{2}}+\zeta\right)$. In this expression, $\Delta d$ is related to the resolution of the diffractionlimited optical system, and $\zeta$ is the relative depth of the excitation beam minimum $(\zeta \ll 1)$. The resolution, defined here as the full width at half-maximum (FWHM) of the registered intensity dip, in the GSD microscope is well 
(a)
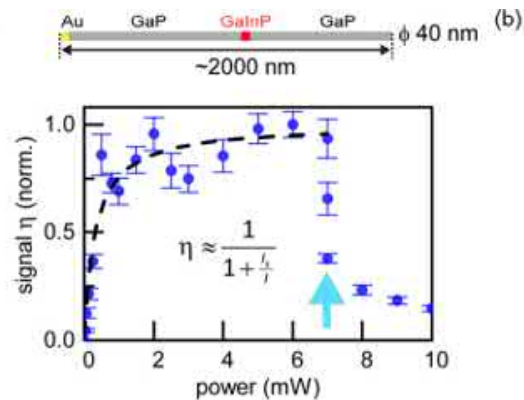

(c)

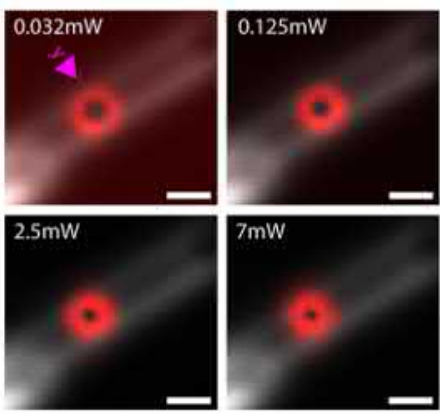

(b)

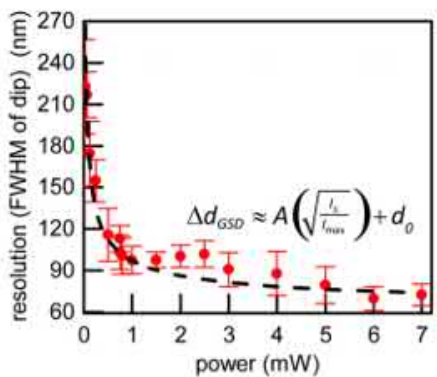

(d)

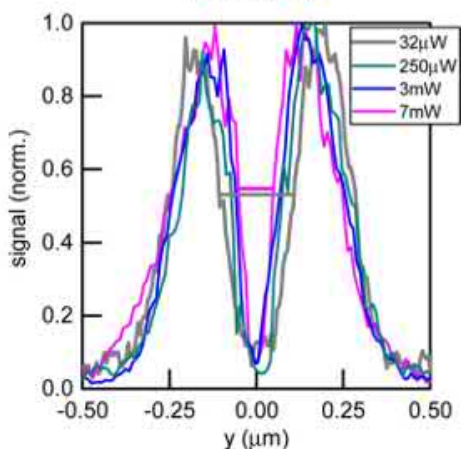

Figure 2. Resolution increase in GSD photoluminescence nanoscopy for a nanowire with a single GaInP segment. (a) Saturation behavior of the GaInP photoluminescence signal $\eta$. The saturation power is $P_{\mathrm{S}}=0.322 \mathrm{~mW}$ (corresponding to a peak threshold intensity $I_{\mathrm{S}} \approx 400 \mathrm{MW} / \mathrm{cm}^{2}$ ). The blue arrow indicates abrupt, irreversible decreases in photoluminescence with three subsequent measurements at $7 \mathrm{~mW}$ shown. Error bars indicate standard deviations of maximal signal from several adjacent line profiles. (b) Resolution scaling $\Delta d_{\mathrm{GSD}}$ as a function of the excitation power. The resolution was defined as the full width at half-maximum (FWHM) of the signal dip. Experimental data and fits to the shown expressions are displayed in (a) and (b) (including a finite offset related to the physical segment diameter in b). Error bars indicate standard deviations from several adjacent line profiles. (c) Scattering (gray) and photoluminescence (red) signal from a single NW. Scale bars: 500 nm. (d) Photoluminescence intensity profiles showing resolution changes as a function of the excitation power.

approximated by $\Delta d_{\mathrm{GSD}} \approx \Delta d \sqrt{\frac{I_{\mathrm{s}}}{I_{\max }}+\zeta}$ and tends to very small values for increasing intensity. In practice, the resolution is limited by the material properties and the residual intensity in the doughnut minimum (details in Supporting Information).

We found that, despite the short exciton lifetime of several picoseconds, it is possible to shelve electrons in the "on" state and ensure the PL of GaInP (Figure 2a). We imaged NWs that contained a single GaInP segment to characterize the signal $\eta$ and the resolution $\Delta d_{\mathrm{GSD}}$ as a function of excitation power. All experiments were performed on a home-built GSD microscope with short excitation pulses ( $\sim 5$ ps in the focal plane) in timegated operation (Supporting Information). On the basis of experimental data (Figure 2a), we inferred a peak threshold intensity $I_{\mathrm{s}} \approx 400 \frac{\mathrm{MW}}{\mathrm{cm}^{2}}$. Having identified the PL properties of GaInP (Figures 1 and 2a), the resolution of the imaging system was significantly improved by adopting a doughnut-shaped excitation beam (Figure $2 b-d$ ). For the excitation wavelength $\lambda$ $=700 \mathrm{~nm}$, the resolution was improved to values comparable to the GaInP segment size $(\sim 50 \mathrm{~nm})$. PL intensity profiles orthogonal to the NW axis (Figure 2d), that is, across the narrow dimension (labeled as $y$ ), were used to estimate the resolution scaling with excitation power (Figure $2 \mathrm{~b}, \mathrm{~d}$ ). Note that our measurements were carried out with a single NW width of $\sim 50 \mathrm{~nm}$ (and this offset was taken into account in the fitting analysis), meaning that resolution will not tend to zero for very large powers. In addition, the dip of the doughnutshaped profile is narrower in FWHM than a Gaussian-like standard excitation profile for the same wavelength (compare Figures $2 \mathrm{~b}$ and $3 \mathrm{~g}$ ). Occasionally, we observed a permanent reduction of the PL signal (to a lower PL level, or even complete loss of signal) starting at powers of $\sim 3-7 \mathrm{~mW}$ (for example, the arrow in Figure 2a). The PL reduction was often accompanied by an increased contrast of the registered minimum (Figure S6).

Next, we proceeded to explore the imaging of barcode $\mathrm{NWs}^{22}$ (Figures 3-5). We imaged NWs that were synthesized to have a diameter of $\sim 20 \mathrm{~nm}$ and consisted of four GaInP PL segments $(233 \pm 40 \mathrm{~nm}$ length) separated by nonluminescent $\mathrm{GaP}$ segments $(164 \pm 21 \mathrm{~nm})$, as characterized by SEM. Figure $3 \mathrm{a}-\mathrm{c}$ provides example confocal and GSD image comparisons. The confocal microscopy mode (Figure 3a) did not resolve individual segments, even after the application of deconvolution algorithms incorporating the known PSF (Figure 3d). GSD nanoscopy (Figure 3b,c,e) clearly distinguished the four segments. We retrieved a more intuitive image representation (Figure 3c) by simple subtraction of the raw data from its lowfrequency counterpart (obtained by low-pass filtering in the frequency domain to remove high-resolution information; see Supporting Information). In principle, the corresponding confocal data can also be used to provide the reference image for subtraction. On the basis of this image (Figure 3c), the lengths of the $\mathrm{GaP}$ and $\mathrm{GaInP}$ segments appeared as $162 \pm 20$ $\mathrm{nm}$ and $225 \pm 11 \mathrm{~nm}$, respectively, in an intensity profile along the NW axis (Figure 3f, direction labeled $x$; lengths were measured as FWHMs based on spacings of maxima and minima; standard deviations from $n=12$ and 9 segments, respectively). Providing a convenient target to estimate the resolution on the thinnest spatial feature, the intensity profile across the NW, that is, orthogonal to the NW axis (Figure 3g, 


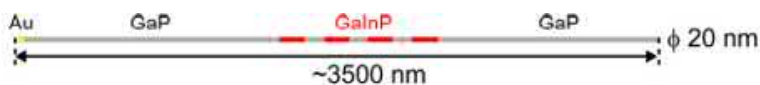

(a) Confocal (raw data)

(b) GSD (raw data)

(c) GSD (linear subtraction)
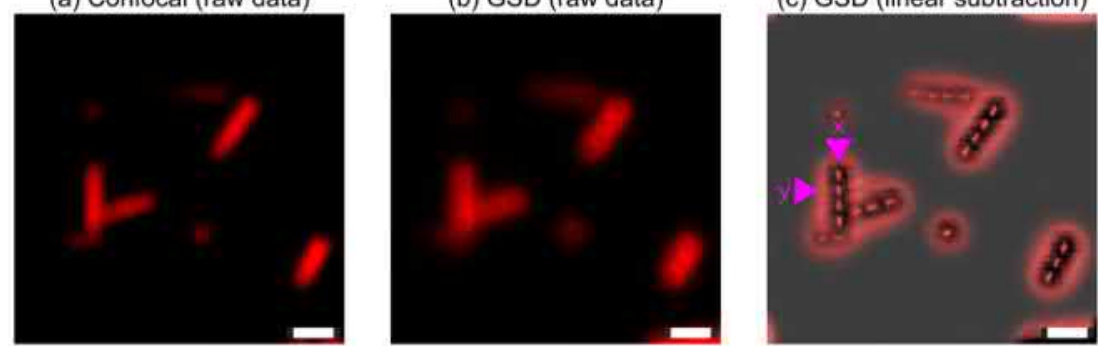

(d) Confocal+ (deconvolution)

(e) GSD+ (deconvolution)
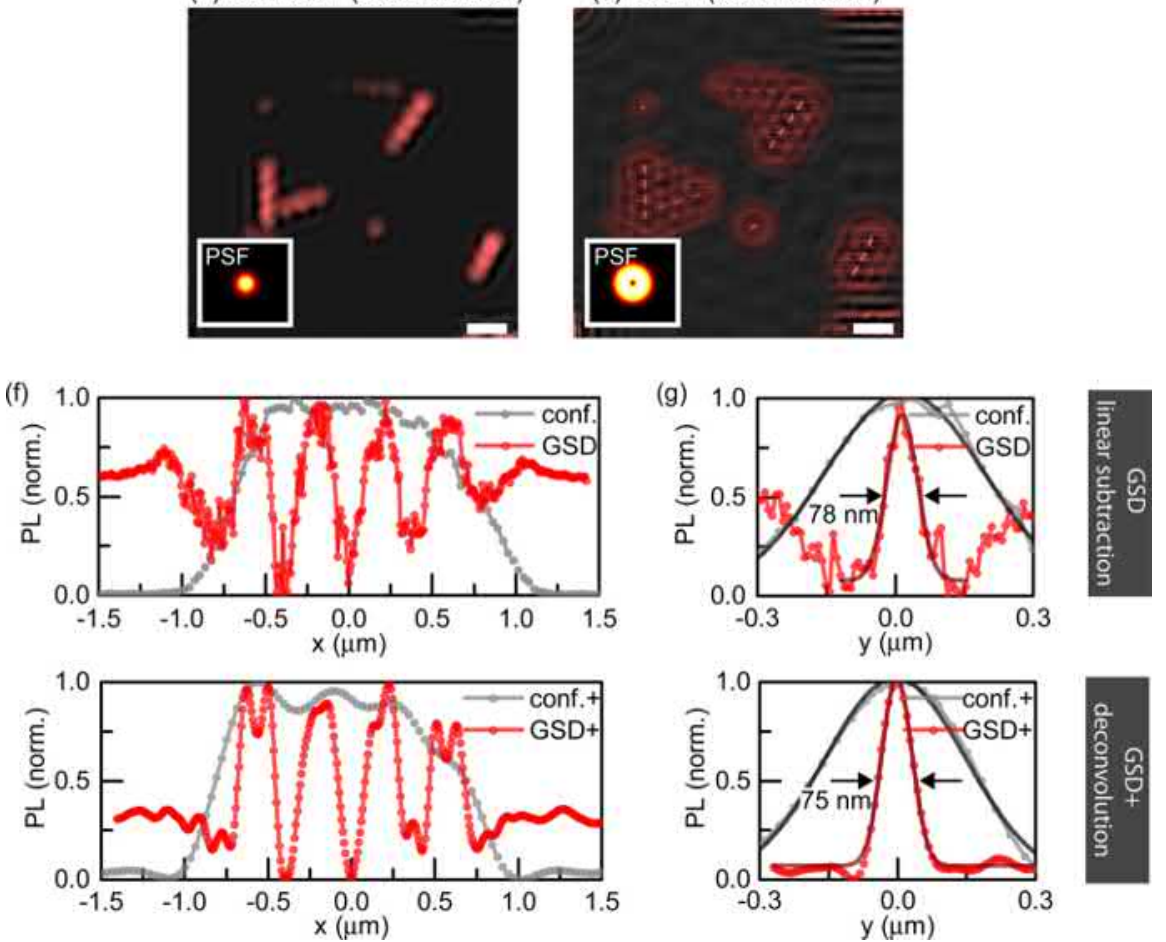

Figure 3. Photoluminescence nanoscopy of barcode nanowires $(\phi=20 \mathrm{~nm})$ with long photoluminescent segments. (a) Nanowires imaged in confocal mode with an excitation wavelength of $\lambda=700 \mathrm{~nm}$ (b) The same nanowires imaged with GSD PL nanoscopy (excitation power $5 \mathrm{~mW}$, excitation wavelength $\lambda=700 \mathrm{~nm}$ ). (c) Restored image by subtraction of raw-data GSD image from its low-frequency counterpart (not shown). (d,e) Reconstructed confocal (d) and GSD (e) image by Wiener deconvolution algorithm with PSFs (insets) calculated analytically. (f,g) PL intensity line profiles along $(x)$ and across $(y)$ a NW to compare confocal and GSD images for (a,c) (top) and (d,e) (bottom), showing the resolution improvement in GSD nanoscopy. All scale bars: $1 \mu \mathrm{m}$.

direction labeled $y$ ) was reduced from $388 \pm 25 \mathrm{~nm}$ in confocal mode to $76 \pm 11 \mathrm{~nm}$ in GSD $(n=12)$. The inferred resolution enhancement by 5 -fold therefore closely matches the modeled PSF of $\sim 66 \mathrm{~nm}$ at $5 \mathrm{~mW}$ (details in Supporting Information section "Limitations of direct subdiffraction imaging with GSD”).

Moving to shorter and more closely spaced GaInP segments (Figure 4), we imaged NWs whose segment dimensions were characterized using SEM to $64 \pm 15 \mathrm{~nm} / 50 \pm 20 \mathrm{~nm}$ for $\mathrm{GaInP} / \mathrm{GaP}$ lengths, respectively, with a diameter $\phi=43 \pm 3$ $\mathrm{nm}$ (Supporting Information, "Specification of NWs" section). The relatively large uncertainties for the segment length measurements are due to low contrast between GaP/GaInP segments in SEM images, as well as possible differences across the population of NWs related to the growth procedure. GSD nanoscopy resolved individual luminescent segments (Figure
$4 \mathrm{~b}-\mathrm{d})$ with an average separation of their centers of $112 \pm 13$ $\mathrm{nm}(n=20$ segments; SEM, $114 \pm 5 \mathrm{~nm} n=32$ segments $)$. Post-deconvolution (Figure 4d,f), we characterized the segment lengths to be $66 \pm 10 \mathrm{~nm} / 50 \pm 16 \mathrm{~nm}(\mathrm{GaInP} / \mathrm{GaP}, n \geq 15)$, observing an excellent agreement with the SEM measurements. While contrast was high $(>0.8)$ for an isolated segment (Figure $2 c, d)$, multiple closely spaced segments were imaged at significantly reduced contrast $(<0.22)$. For the nanowires of Figure 4, we found that the contrast continuously decreased between adjacent GaInP segments along a nanowire (compare enlarged views in Figure 4e), possibly related to a gradation in inherent photoluminescence strength. ${ }^{35}$ The GaInP segment with the lowest photoluminescence corresponds to the first grown segment. $^{22}$ Moreover, in GaInP nanowires, reduced contrast can arise from differences in material composition 
(a) Confocal (raw data)

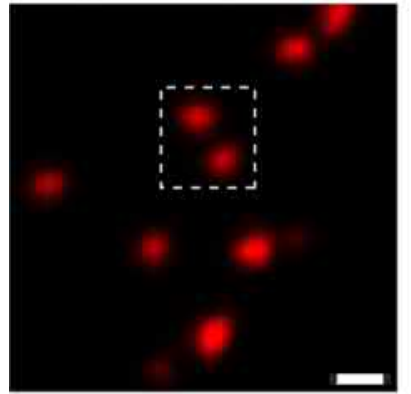

(d) GSD+ (deconvolved)

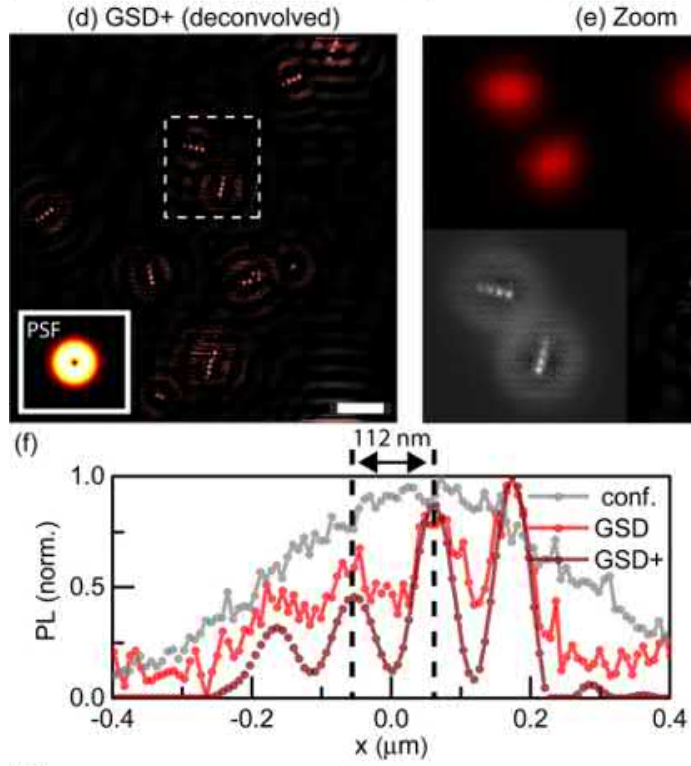

(h)
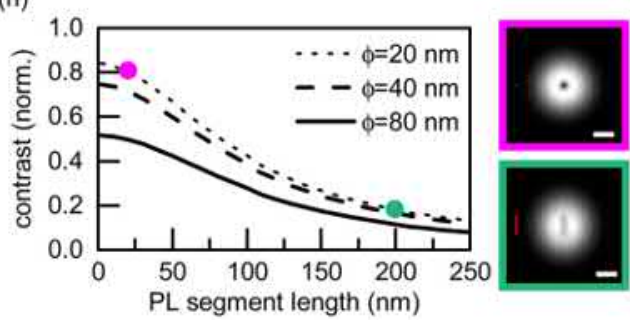

(b) GSD (raw data)

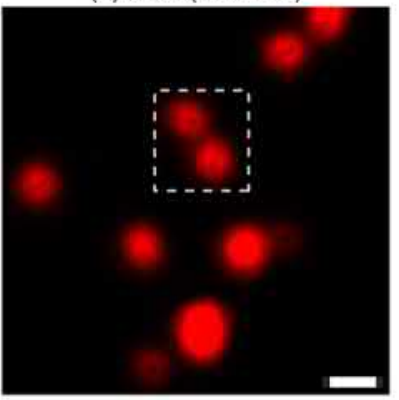

(e) Zoom (c) GSD (subtraction)
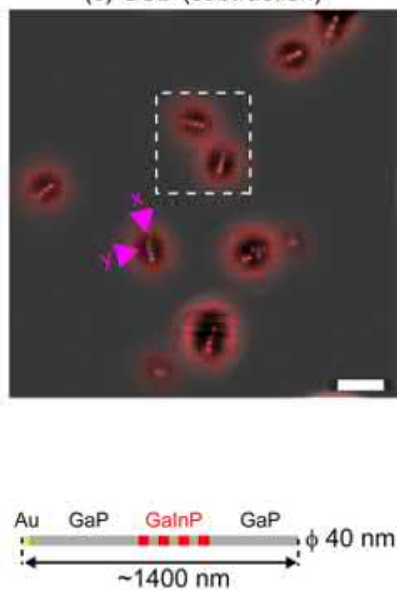

(g)

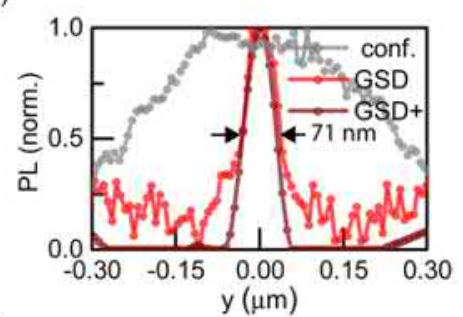

(i)

Figure 4. Photoluminescence nanoscopy of barcode nanowires $(\phi=40 \mathrm{~nm})$ with short luminescent segments. (a) Nanowires imaged in confocal mode. Excitation wavelength $\lambda=700 \mathrm{~nm}$. (b) The same area imaged by GSD PL nanoscopy with excitation power 3 mW. (c) Restored image by subtraction of raw-data GSD image from its low-frequency counterpart (not shown). (d) Reconstructed GSD image by Wiener deconvolution algorithm with PSF (inset) calculated analytically. (e) Enlarged views of selected image regions from (a-d) (GSD subtraction and deconvolved shown in greyscale for better visualization of achieved contrast). (f,g) Intensity profile lines along $(x)$ and across $(y)$ a NW indicated by pink arrows. (h) Simulated contrast for GSD imaging of an extended PL segment versus photoluminescence segment length (for different NW diameters $\phi$ ). (i) Simulated imaging contrast dependence on separation between luminescent segments (at the middle segment). The modeled NW consists of three photoluminescence segments with diameter and length equal $20 \mathrm{~nm}$. Images in (h,i) show the images calculated as a convolution of the objects (red) with the modeled PSF for $5 \mathrm{~mW}$ excitation power. Scale bars $1 \mu \mathrm{m}(\mathrm{a}-\mathrm{d}), 200 \mathrm{~nm}(\mathrm{e}, \mathrm{h}, \mathrm{i})$.

along the length of the nanowires, which affect the intensity, as previously described. ${ }^{22,36}$

Modeling of the GSD image formation revealed a clear connection of image contrast with the PL segment length and diameter (Figure 4h), with highest contrasts for shortest and thinnest PL segments. In addition, small spacings between segments lead to decreased contrast (Figure 4i). Therefore, the observed contrast enhancement upon the sudden decrease in PL signal (Figure S6) suggests a volume reduction of the emissive GaInP segment. As size and spacing strongly affect contrast, there is clearly room for optimization in the design of heterostructures to increase the quality of images, keeping in mind that smaller PL volumes come with reduced signals. Figure 5 displays further data on $40 \mathrm{~nm}$ diameter NWs with short luminescent segments $(50 \pm 8 \mathrm{~nm})$, this time with $\sim 2$ times longer $\mathrm{GaP}$ spacers $(95 \pm 13 \mathrm{~nm})$. The contrast along the NWs increased from 0.10 for the nanowires in Figure 4 to 0.14 for the nanowires in Figure 5 (data not shown). We compared the GSD nanoscopy characterization of the nanowires to SEM images (Figure $5 \mathrm{c}-\mathrm{h}$ ) and found an excellent agreement in the segment diameters $\langle\Delta y\rangle$, lengths $\langle\Delta x\rangle$, and separations $\left\langle\Delta x_{\mathrm{c}}\right\rangle$, extracted using both methods (data tabulated in Figure 5). 


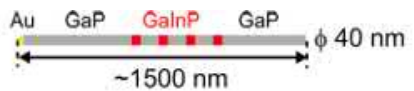

Photoluminescence microscopy
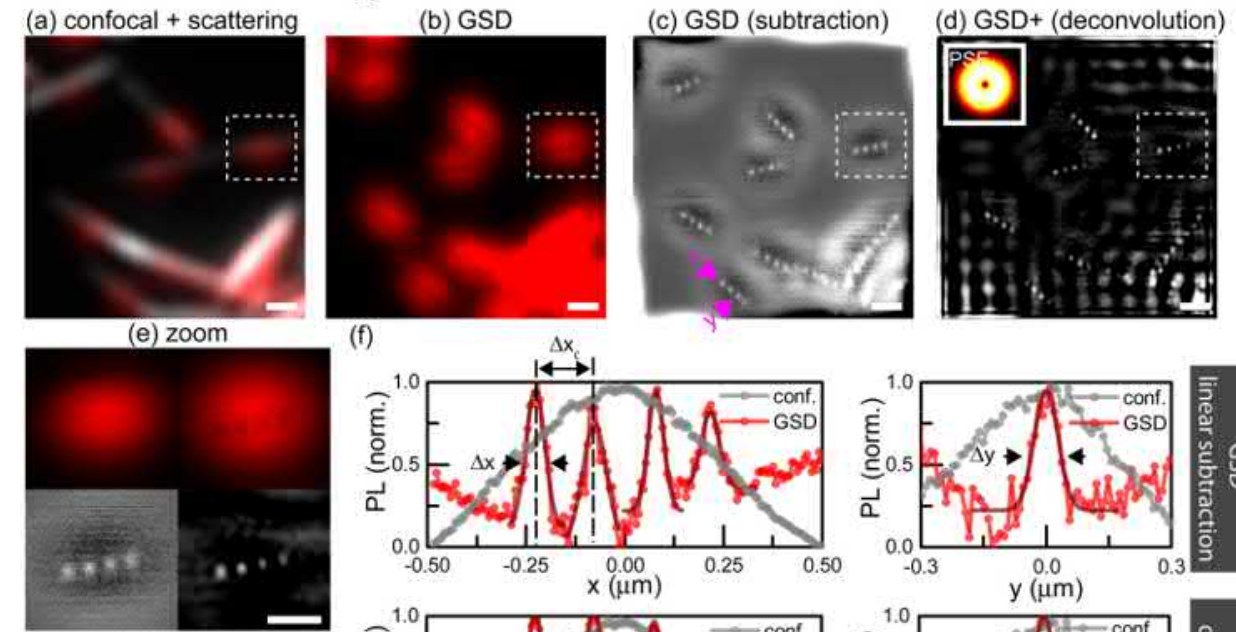

(d) GSD+ (deconvolution)

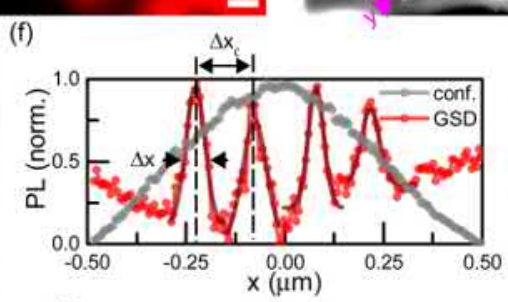

GSD (subtraction)

$\left\langle\Delta x_{c}\right\rangle=147 \pm 14 \mathrm{~nm}$ $\langle\Delta x\rangle=69 \pm 6 \mathrm{~nm}$ $\langle\Delta y\rangle=74 \pm 12 \mathrm{~nm}$ GSD+ (deconvolution) $\left\langle\Delta x_{c}\right\rangle=146 \pm 8 \mathrm{~nm}$ $\langle\Delta x\rangle=63 \pm 13 \mathrm{~nm}$ $\langle\Delta y\rangle=62 \pm 9 \mathrm{~nm}$
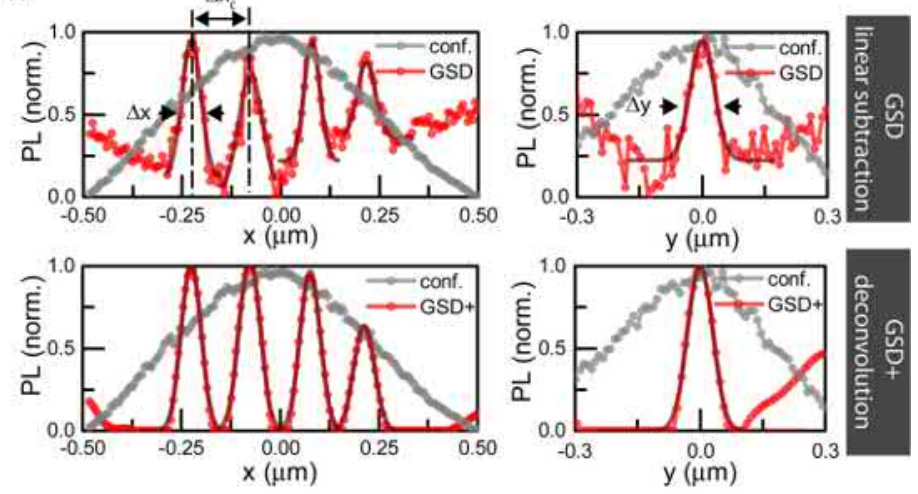

Scanning electron microscopy

(g)

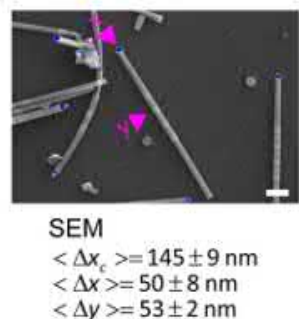

(h)
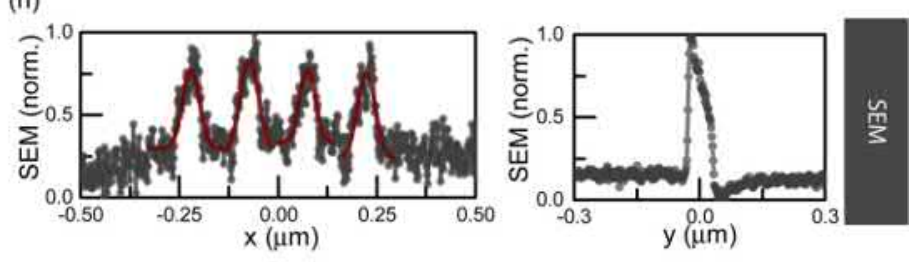

Figure 5. Photoluminescence nanoscopy of barcode nanowires $(\phi=40 \mathrm{~nm})$ with short luminescent segments and larger spacers compared to NWs in Figure 4 to increase the contrast. (a) Nanowires imaged in confocal mode (excitation wavelength $\lambda=700 \mathrm{~nm}$ ). (b) The same area imaged by GSD PL nanoscopy (excitation power, $5 \mathrm{~mW}$ ). (c) Restored image by linear subtraction. (d) Deconvolved GSD image by Wiener deconvolution. The inset shows the PSF. (e) Enlarged view of selected image regions from (a-d). (f) Intensity profile lines along (x) and across $(y)$ the NW indicated by pink arrows in (c). (g) Nanowires imaged by scanning electron microscopy (SEM). (h) Intensity profile lines along ( $x$ ) and across $(y)$ the NW indicated by pink arrows in $(\mathrm{g})$. Symbols: $\Delta x_{\mathcal{v}}$, separation of adjacent maxima; $\Delta x$, length of segment; $\Delta y$, width of segment. Data tabulated in the bottom left margin compare GSD and SEM results for NWs from the same batch for more than seven segments. Scale bars $500 \mathrm{~nm}$ (a-d), $200 \mathrm{~nm}$ $(\mathrm{e}, \mathrm{g})$.

In summary, we have demonstrated experimentally that GSD nanoscopy resolves $\mathrm{GaP} / \mathrm{GaInP} \mathrm{NW}$ heterostructures with diameters down to $20 \mathrm{~nm}$ at a 5 -fold resolution enhancement compared to confocal microscopy. GaP/GaInP NWs with even smaller diameters $(10 \mathrm{~nm})$ became challenging to image due to significant emission intermittency. As the diameter of NWs approached the exciton size (GaInP $\sim 4-10 \mathrm{~nm}$ ), the PL showed strong fluctuations (Figure S5 in Supporting Information), making segment positions virtually impossible to extract. We note that PL fluctuations can be possibly controlled $^{37,38}$ by choosing the PL material, notably through exciton-size or core-shell engineering to protect surface excitons. However, the nature of signal intermittencies would not allow the direct application of a PALM/STORM/GSDIM strategy: ${ }^{26-28,39}$ Excitons from an individual segment cannot be made to emit together while keeping the neighboring segment "silent", because the electrons behave independently and cannot be "locked" into one of the two states for an extended time. Even if selection ("activation") at the single-exciton level were realized (e.g., by very low fluxes of excitation photons), the exciton would not deliver bursts of $\gg 1$ consecutive photons, which is a fundamental requirement to allow the localization of one entity (here, the segment).

Further, it is worth commenting on a relevant difference between methods that prepare the "on" state at the targeted coordinate(s) ("positive" imaging) and those "inverted" approaches, as applied here, which locally single out the "off" state ("negative" imaging). In both cases, coordinates are targeted by positioning a zero or zeros (minimum/a) of the state-switching light intensity. In "positive" imaging, signals 
from the locally prepared "on"-state features are not affected by any contributions from neighboring non-targeted features because these are in an "off" state. But the "negative"-imaging modalities, forcing the emissive "on" state everywhere except at the zero(s), suffer from the problem of collecting signal from the entire diffraction zone. This includes contributions from all nearby features, whose signals fill up the all-important dip in registered intensity, partially hampering super-resolution information in the presence of noise. Note that the limitation does not stem from an imperfect state preparation. The "negative" modality is just as efficient in generating the "on"-"off" state contrast in the specimen as the "positive" modality, but the readout/detection step is less favorable in terms of contrast. The effect of reduced contrast in the presence of nearby emitters was previously appreciable in GSD nanoscopy data recorded from nitrogen vacancy $(\mathrm{NV})$ color centers, that is, confined point-like emitters in diamond. ${ }^{32}$ In the extended semiconductor structures investigated here, we showed that shorter PL segments lead to higher contrast, as do larger separations from the nearest luminescent neighbors. Certainly, the ability to freely control the geometry within NW heterostructures (notably, the sequence of PL segment lengths and spacings) allows for further optimization. Another possible direction for improvements will be controlled changes in the NW segments' material composition. This would allow for the modification of spectral characteristics (even customized differences among adjacent PL segments) as well as saturation behaviors and damage thresholds. The latter remains a limiting factor to additional resolution improvements, and a better understanding of the mechanisms of PL loss will benefit further advances.

Because of the far-red excitation wavelength (low phototoxicity) and the moderate average power, we believe the described approach will be highly relevant for revealing positions, orientations, and identities of NWs in biological contexts. Membranes or other cellular structures could be visualized by complementary nanoscale imaging methods such as STED. The good performance of NW GSD nanoscopy at room temperature indicates similar applicability at physiological temperatures under live-cell conditions. The comparatively low excitation power required to obtain a substantial resolution enhancement over confocal imaging should allow parallelization of this approach, which may significantly increase the speed of nanoscale imaging in the future.

\section{ASSOCIATED CONTENT}

\section{S Supporting Information}

The Supporting Information is available free of charge on the ACS Publications website at DOI: 10.1021/acs.nanolett.7b00468.

Experimental setup, synthesis of $\mathrm{GaP} / \mathrm{GaInP}$ barcode nanowires, sample preparation, mathematical image processing, limitations of direct subdiffraction imaging with GSD (resolution and contrast), specifications of NWs, lookup tables (color scales) (PDF)

\section{AUTHOR INFORMATION}

\section{Corresponding Authors}

*E-mail: joanna.oracz@mpibpc.mpg.de.

*E-mail: christelle.prinz@ftf.lth.se.

*E-mail: stefan.hell@mpibpc.mpg.de.
ORCID

Stefan W. Hell: 0000-0002-9638-5077

\section{Author Contributions}

The manuscript was written through contributions of all authors. All authors have given approval to the final version of the manuscript.

\section{Funding}

This work was supported by the Foundation for Polish Science International Ph.D. Projects Programme, cofinanced by the EU European Regional Development Fund. The Lund team acknowledges funding from NanoLund, the Swedish Research Council (VR) and the ERC consolidator grant NanoPokers (682206) and thank Nicklas Anttu for help with nanowire characterization.

\section{Notes}

The authors declare no competing financial interest.

\section{ABBREVIATIONS}

GaInP, gallium indium phosphide; GaP, gallium phosphide; GSD, ground state depletion; NW, nanowire; NV, nitrogen vacancy; PL, photoluminescence; PSF, point spread function; SEM, scanning electron microscopy; STED, stimulated emission depletion; QD, quantum dot

\section{REFERENCES}

(1) Tian, B.; Cohen-Karni, T.; Qing, Q.; Duan, X.; Xie, P.; Lieber, C. M. Science 2010, 329, 830-834.

(2) Qing, Q.; Jiang, Z.; Xu, L.; Gao, R.; Mai, L.; Lieber, C. M. Nat. Nanotechnol. 2013, 9 (2), 142-147.

(3) Eschermann, J. F.; Stockmann, R.; Hueske, M.; Vu, X. T.; Ingebrandt, S.; Offenhäusser, A. Appl. Phys. Lett. 2009, 95 (8), 083703.

(4) Liu, H.; He, J.; Tang, J.; Liu, H.; Pang, P.; Cao, D.; Krstic, P.; Joseph, S.; Lindsay, S.; Nuckolls, C. Science 2010, 327 (5961), 64-67.

(5) Williams, K. A.; Veenhuizen, P. T. M.; de la Torre, B. G.; Eritja, R.; Dekker, C. Nature 2002, 420 (6917), 761-761.

(6) Bisker, G.; Dong, J.; Park, H. D.; Iverson, N. M.; Ahn, J.; Nelson, J. T.; Landry, M. P.; Kruss, S.; Strano, M. S. Nat. Commun. 2016, 7, 10241.

(7) Patolsky, F.; Timko, B. P.; Yu, G.; Fang, Y.; Greytak, A. B.; Zheng, G.; Lieber, C. M. Science 2006, 313 (5790), 1100-1104.

(8) Robinson, J. T.; Jorgolli, M.; Shalek, A. K.; Yoon, M.-H.; Gertner, R. S.; Park, H. Nat. Nanotechnol. 2012, 7 (3), 180-184.

(9) Fakhri, N.; Wessel, A. D.; Willms, C.; Pasquali, M.; Klopfenstein, D. R.; MacKintosh, F. C.; Schmidt, C. F. Science 2014, 344 (6187), 1031-1035.

(10) Smith, B. R.; Ghosn, E. E. B.; Rallapalli, H.; Prescher, J. A.; Larson, T.; Herzenberg, L. A.; Gambhir, S. S. Nat. Nanotechnol. 2014, 9 (6), 481-487.

(11) Xu, A. M.; Aalipour, A.; Leal-Ortiz, S.; Mekhdjian, A. H.; Xie, X.; Dunn, A. R.; Garner, C. C.; Melosh, N. A. Nat. Commun. 2014, 5, 3613.

(12) Hällström, W.; Lexholm, M.; Suyatin, D. B.; Hammarin, G.; Hessman, D.; Samuelson, L.; Montelius, L.; Kanje, M.; Prinz, C. N. Nano Lett. 2010, 10 (3), 782-787.

(13) Cellot, G.; Cilia, E.; Cipollone, S.; Rancic, V.; Sucapane, A.; Giordani, S.; Gambazzi, L.; Markram, H.; Grandolfo, M.; Scaini, D.; Gelain, F.; Casalis, L.; Prato, M.; Giugliano, M.; Ballerini, L. Nat. Nanotechnol. 2009, 4 (2), 126-133.

(14) Piret, G.; Perez, M.-T.; Prinz, C. N. ACS Appl. Mater. Interfaces 2015, 7 (34), 18944-18948.

(15) Prinz, C. N. J. Phys.: Condens. Matter 2015, 27 (23), 233103.

(16) Persson, H.; Li, Z.; Tegenfeldt, J. O.; Oredsson, S.; Prinz, C. N. Sci. Rep. 2015, 5, 18535 .

(17) Kim, W.; Ng, J. K.; Kunitake, M. E.; Conklin, B. R.; Yang, P. J. Am. Chem. Soc. 2007, 129 (23), 7228-7229. 
(18) Berthing, T.; Bonde, S.; Sørensen, C. B.; Utko, P.; Nygård, J.; Martinez, K. L. Small 2011, 7 (5), 640-647.

(19) Shalek, A. K.; Robinson, J. T.; Karp, E. S.; Lee, J. S.; Ahn, D.-R.; Yoon, M.-H.; Sutton, A.; Jorgolli, M.; Gertner, R. S.; Gujral, T. S.; MacBeath, G.; Yang, E. G.; Park, H. Proc. Natl. Acad. Sci. U. S. A. 2010, 107 (5), 1870-1875.

(20) Mumm, F.; Beckwith, K. M.; Bonde, S.; Martinez, K. L.; Sikorski, P. Small 2013, 9 (2), 263-272.

(21) Berthing, T.; Bonde, S.; Rostgaard, K. R.; Madsen, M. H.; Sørensen, C. B.; Nygård, J.; Martinez, K. L. Nanotechnology 2012, 23 (41), 415102.

(22) Adolfsson, K.; Persson, H.; Wallentin, J.; Oredsson, S.; Samuelson, L.; Tegenfeldt, J. O.; Borgström, M. T.; Prinz, C. N. Nano Lett. 2013, 13 (10), 4728-4732.

(23) Mattsson, K.; Adolfsson, K.; Ekvall, M. T.; Borgström, M. T.; Linse, S.; Hansson, L.-A.; Cedervall, T.; Prinz, C. N. Nanotoxicology 2016, 10 (8), 1160-1167.

(24) Hell, S. W. Science 2007, 316 (5828), 1153-1158.

(25) Huang, B.; Babcock, H.; Zhuang, X. Cell 2010, 143 (7), 10471058.

(26) Betzig, E.; Patterson, G. H.; Sougrat, R; Lindwasser, O. W.; Olenych, S.; Bonifacino, J. S.; Davidson, M. W.; Lippincott-Schwartz, J.; Hess, H. F. Science 2006, 313 (5793), 1642-1645.

(27) Hess, S. T.; Girirajan, T. P. K.; Mason, M. D. Biophys. J. 2006, 91 (11), 4258-4272.

(28) Rust, M. J.; Bates, M.; Zhuang, X. Nat. Methods 2006, 3 (10), 793-796.

(29) Hell, S. W.; Wichmann, J. Opt. Lett. 1994, 19 (11), 780-782.

(30) Hell, S. W.; Kroug, M. Appl. Phys. B: Lasers Opt. 1995, 60 (5), 495-497.

(31) Bretschneider, S.; Eggeling, C.; Hell, S. W. Phys. Rev. Lett. 2007, 98 (21), 218103.

(32) Rittweger, E.; Wildanger, D.; Hell, S. W. EPL 2009, 86 (1), 14001.

(33) Heintzmann, R.; Jovin, T. M.; Cremer, C. J. Opt. Soc. Am. A 2002, 19 (8), 1599-1609.

(34) Gustafsson, M. G. L. Proc. Natl. Acad. Sci. U. S. A. 2005, 102 (37), 13081-13086.

(35) Bao, W.; Melli, M.; Caselli, N.; Riboli, F.; Wiersma, D. S.; Staffaroni, M.; Choo, H.; Ogletree, D. F.; Aloni, S.; Bokor, J.; Cabrini, S.; Intonti, F.; Salmeron, M. B.; Yablonovitch, E.; Schuck, P. J.; WeberBargioni, A. Science 2012, 338 (6112), 1317-1321.

(36) Berg, A.; Lenrick, F.; Vainorius, N.; Beech, J. P.; Wallenberg, R. L.; Borgström, M. T. Nanotechnology 2015, 26 (43), 435601.

(37) Frantsuzov, P.; Kuno, M.; Janko, B.; Marcus, R. A. Nat. Phys. 2008, 4 (5), 519-522.

(38) Glennon, J. J.; Tang, R.; Buhro, W. E.; Loomis, R. A. Nano Lett. 2007, 7 (11), 3290-3295.

(39) Fölling, J.; Bossi, M.; Bock, H.; Medda, R.; Wurm, C. A.; Hein, B.; Jakobs, S.; Eggeling, C.; Hell, S. W. Nat. Methods 2008, 5 (11), 943-945. 


\title{
Supporting Information
}

\section{Ground State Depletion Nanoscopy Resolves Semiconductor Nanowire Barcode Segments at Room Temperature}

\author{
Joanna Oracz, ${ }^{\dagger}+$ Karl Adolfsson, ${ }^{\S}$ Volker Westphal, ${ }^{\dagger}$ Czestaw Radzewicz, ${ }^{+}$ \\ Magnus T. Borgström, ${ }^{\S}$ Steffen J. Sahl,$^{\dagger}$ Christelle N. Prinz ${ }^{\S} \&$ Stefan W. Hell ${ }^{\dagger}$
}

†Department of NanoBiophotonics, Max Planck Institute for Biophysical Chemistry, 37077 Göttingen, Germany

\#aculty of Physics, University of Warsaw, 02-093 Warsaw, Poland

${ }^{\S}$ Division of Solid State Physics and NanoLund, Lund University, 22100 Lund, Sweden

\section{Table of Contents}

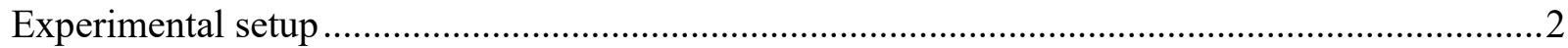

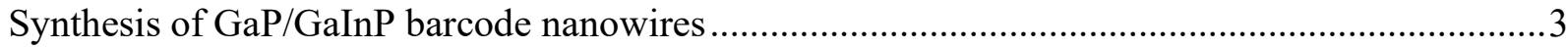

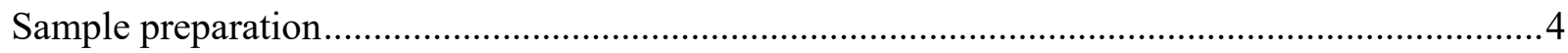

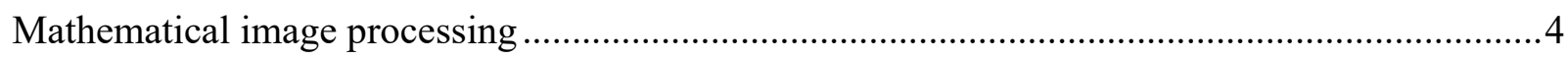

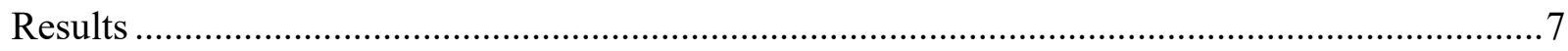

Limitations of direct subdiffraction imaging with GSD (resolution and contrast) ........................ 8

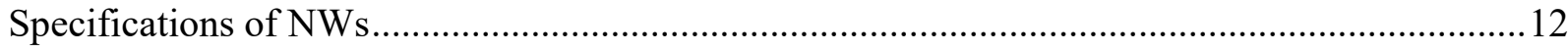

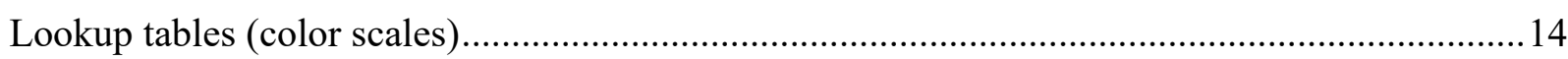

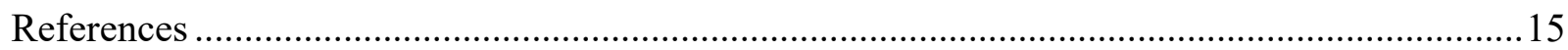




\section{Experimental setup}

Measurements were performed on a home-built microscopy system equipped with a commercial Ti:Sapphire oscillator (Mai Tai, Spectra Physics), a piezo scanner (NanoMax MAX312D, Melles Griot) and a high numerical aperture objective (NA 1.4, 100×, Leica). A schematic drawing of the setup is presented in Figure S1a. A doughnut-shaped excitation profile in the focal plane was generated by a helical phase plate (HPP, VPP-1a, Rochester Photonics). Confocal recordings were performed without the HPP. Nanowires were excited at $\sim 700 \mathrm{~nm}$ by $\sim 5 \mathrm{ps}$ laser pulses stretched in a polarization-maintaining single-mode fiber. The pulse repetition rate was $80 \mathrm{MHz}$. Photoluminescence was filtered by bandpass/cut-off filters (AHF Analysentechnik, Semrock) and detected by a single-photon counting avalanche photodiode (APD, SPCM-AQR-13-FC, Perkin Elmer). In some experiments, the APD was replaced by a single-photon counting module with high temporal resolution (low dead time) (id100, idQuantique). Confocal detection was realized by a multimode fiber (M31LO3, Thorlabs) with $\sim 1$ Airy diameter. The photoluminescence lifetime was recorded with a time-correlated single-photon counting module (SPC-150, Becker \& Hickl). The response function of the detection electronics (jitter) was measured by scattering of an ultrashort laser pulse ( $\sim 128 \mathrm{fs}$ ) to be $40 \mathrm{ps}$ (Figure 1d). Typical excitation powers for the recording of superresolution GSD images varied from $0.5 \mathrm{~mW}$ to $7 \mathrm{~mW}$. All powers stated were measured at the back aperture of the objective lens. Pixel dwell times were adjusted to be $\sim 0.1-1 \mathrm{~ms}$. All optical experiments were carried out at room temperature. 
(a)

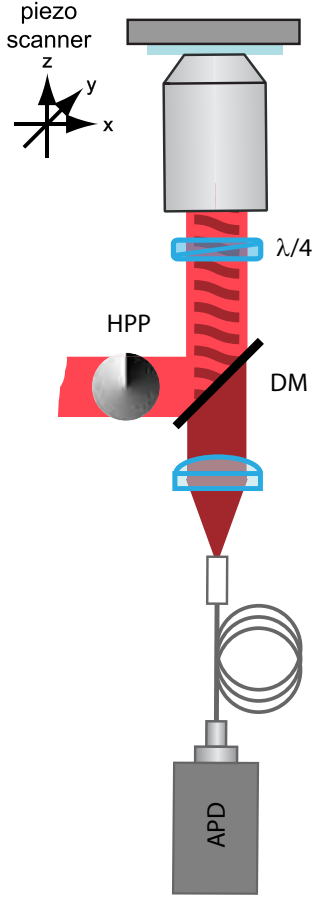

(b)
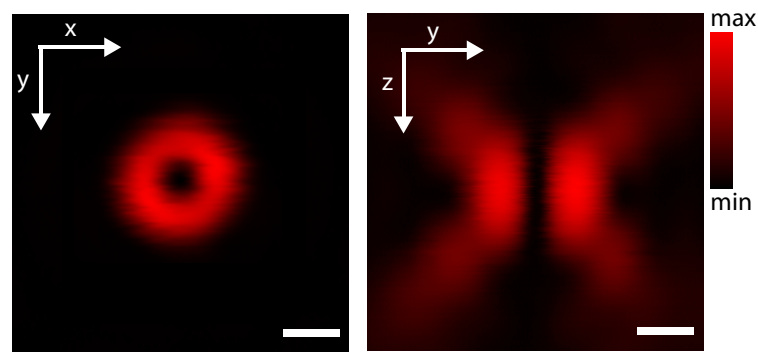

(c)

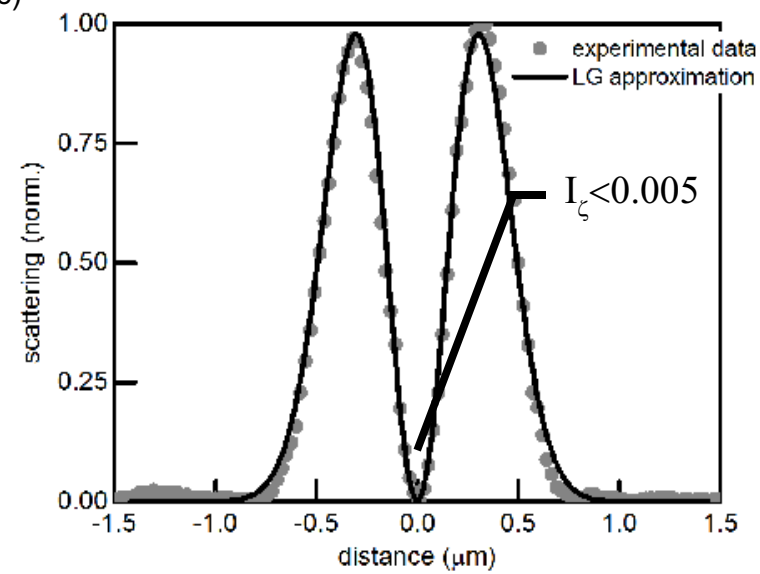

Figure S1. (a) Experimental setup for GSD nanoscopy (DM: dichroic mirror, APD: avalanche photodiode, HPP: helical phase plate, $\lambda / 4$ : quarter-wave plate). The scattered signal could be separately measured on a photomultiplier tube by reflection with a pellicle beamsplitter (path not shown). (b) Point spread function of the microscope, as measured by scattering of $80 \mathrm{~nm}$ gold beads in reflection. Scale bars: $500 \mathrm{~nm}$. (c) Zero quality of the doughnut-shaped excitation beam measured to be $I_{\zeta}<0.5 \%$ (LG: Laguerre-Gaussian).

\section{Synthesis of GaP/GaInP barcode nanowires}

$\mathrm{GaP} / \mathrm{GaInP}$ nanowires were synthesized by use of Au-particle assisted vapor liquid solid growth ${ }^{1}$ in a commercial MOVPE system (Aixtron 200/4). Catalytic Au particles were deposited on GaP(111)B substrates by means of aerosol deposition ${ }^{2}$, producing particles with diameter of 10 , 20,40 or $80 \mathrm{~nm}$ and at a surface density of 2 or $4 \mu \mathrm{m}^{-2}$. The substrate was then placed in the MOVPE reactor chamber. The epitaxial growth was preceded by a 10 -min temperature annealing step at $650^{\circ} \mathrm{C}$ with a constant $\mathrm{PH}_{3}$ flow (molar fraction of $6.9 \times 10^{-3}$ ) in $\mathrm{H}_{2}$ carrier gas (total flow of $13000 \mathrm{SCCM}$ ) to remove the native oxide from the GaP surface. The temperature was subsequently lowered to $440^{\circ} \mathrm{C}$ and precursors for $\mathrm{GaP}$ were introduced into the reactor chamber, i.e. trimethylgallium (TMGa, at a molar fraction of $\left.4.5 \times 10^{-5}\right)$ and phosphine $\left(\mathrm{PH}_{3}\right.$, at a molar fraction 
of $\left.6.9 \times 10^{-3}\right)$. Hydrogen chloride $(\mathrm{HCl})$, which impedes undesirable radial growth, was also added at a molar fraction of $6.2 \times 10^{-5}$. Segments of GaInP were synthesized by introducing the precursor for indium, trimethylindium (TMIn at a molar fraction of $1.3 \times 10^{-5}$ ). The length and arrangement of GaP and GaInP segments were tuned by opening and closing the TMIn source at different time points. In our experiments, the GaInP growth time was varied between $1 \mathrm{~s}$ and $75 \mathrm{~s}$, resulting in segments from $\sim 50 \mathrm{~nm}$ to $>200 \mathrm{~nm}$ in length. Nanowires with 1 and $4 \mathrm{GaInP}$ segments were synthesized. The GaP growth time was varied between $5 \mathrm{~s}$ and $180 \mathrm{~s}$, resulting in segment lengths between $\sim 100 \mathrm{~nm}$ and $1.4 \mu \mathrm{m}$.

\section{Sample preparation}

The NWs substrate was first vertically placed in an Eppendorf tube filled with Milli-Q water and then sonicated for $\sim 1$ min to detach NWs from their substrate. Next, the dilute NWs suspension was spread on a coverslip (plasma-cleaned) and dried overnight or under a vacuum pump (dropcasting). A small drop of the microscope immersion oil used for imaging (Type B, Cargille) was added to a microscope object slide for index matching, and the coverslip put on top. After oil had spread out under the whole coverslip, the sample was sealed with nail polish.

\section{Mathematical image processing}

The present demonstration of PL GSD nanoscopy extracts high-resolution (sub-diffraction) information by preparing features in the "on" state everywhere except at and near the minimum of a doughnut-shaped excitation beam, resulting in "negative" images at raw data level. This drawback can in principle be overcome experimentally (by applying a second modulated laser beam, which excites non-saturated emitters, and a lock-in detection ${ }^{3}$ ), or by mathematical processing. The second option is easier to implement and does not necessitate slower recording. We therefore used two different methods: linear subtraction and Wiener deconvolution (Figure S2).

For the linear subtraction, we first applied a low-pass Gaussian filter to the original data set (Figure S2a) to remove high-resolution information from the image (Figure S2b). An image was then created by subtraction of original data from its low-resolution copy (Figure S2c: image b minus image a). In principle, a confocal image might be used as substitute for image b, but, due to potential movement of the piezo stage or any instability of the experimental system, the mathematical procedure is preferred. We implemented a Wiener deconvolution algorithm based on division of the raw data (image a) by the point spread function (PSF) of the GSD microscope 
(Figure S2d) in the frequency domain. Deconvolution (Figure S2e) typically gave satisfactory results, however for small-diameter NWs $(\sim 20 \mathrm{~nm})$ the image obtained by our Wiener deconvolution algorithm had substantial artifacts (Figure S2e).

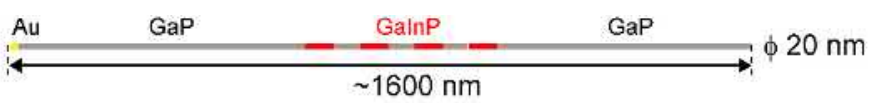

(a) raw data

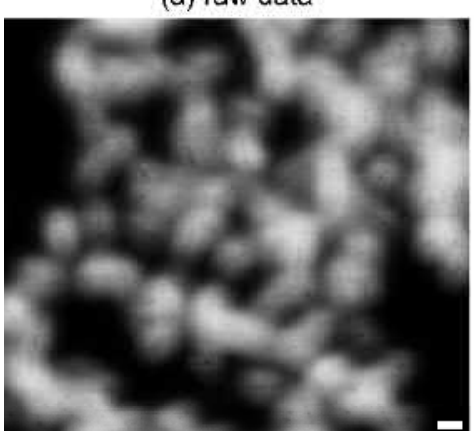

(d) PSF

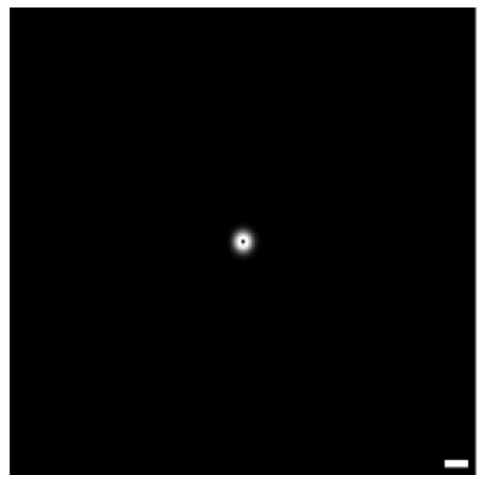

(b) raw data + low pass filter

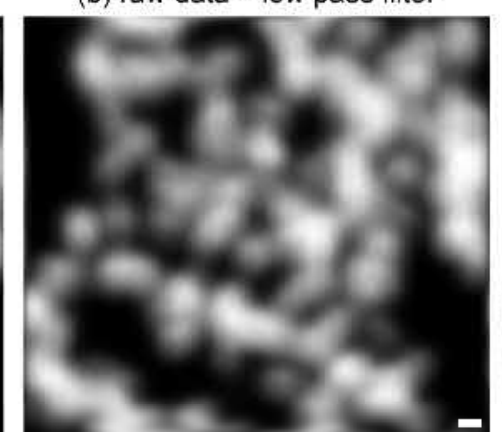

(e) linear deconvolution (c) linear subtraction

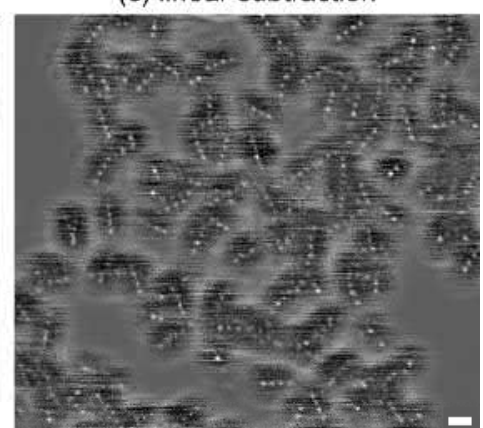

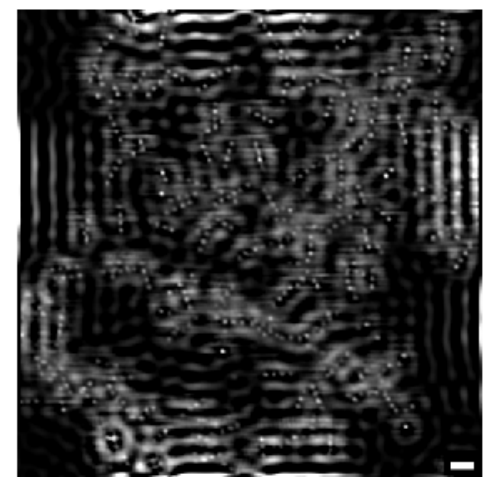

Figure S2. Image restoration. (a) Raw-data image of NW barcode $(\phi=20 \mathrm{~nm})$ with four luminescent segments. The excitation power was $4 \mathrm{~mW}$. (b) Result of low-pass Gaussian filter applied to raw data. (c) Image created by linear subtraction (image b - image a). (d) Modeled point spread function for $4 \mathrm{~mW}$. (e) Image resulting from Wiener deconvolution using the raw-data image (a) and the estimated PSF (d). All scale bars: $500 \mathrm{~nm}$.

The PSF in GSD imaging is dependent on the emitter properties, and is difficult to assess experimentally for extended emitters. The GaInP PL segment size is comparable to the expected resolution, as estimated from the characterized saturation behavior (Figure 2a) and the PSF (Figure $\mathrm{S} 1 b)$. For this reason, we modeled an emitter response function $h_{G S D}$. We calculated the effective PSF using a non-saturated point spread function approximated by a Laguerre-Gaussian beam $h_{P S F}(r)$ (Figure S1c) and the experimentally measured saturation properties (Figure 2a): 


$$
\begin{aligned}
& h_{G S D}\left(r, P_{E x c}\right)=h_{P S F}(r) \otimes h_{o b j}\left(\delta, P_{E x c}\right) \\
& h_{G S D}\left(r, P_{E x C}\right) \approx\left(1+\frac{P_{S}}{P_{E x c} \cdot 2.66\left(\frac{2 r^{2}}{w_{0}^{2}}+\zeta\right) \exp \left(-\frac{2 r^{2}}{w_{0}^{2}}\right)}\right)^{-1}
\end{aligned}
$$

where a point-like object is denoted by $h_{o b j}$, the saturation average power is $P_{\mathrm{S}}=0.322 \mathrm{~mW}$, the average excitation power is $P_{E x c}$, the doughnut zero parameter is denoted by $\zeta=0.001$, and the Gaussian beam width $w_{0}=430 \mathrm{~nm}$.

The effective PSF is a multiplication of $h_{G S D}$ with the confocal detection probability $h_{d e t}$. The detection probability was represented by a Gaussian distribution $\left(w_{0}=280 \mathrm{~nm}\right)$, close to experimental conditions. The final effective PSF $h_{\text {eff }}$ then takes the form:

$$
h_{e f f}\left(r, P_{E x c}\right)=h_{G S D}\left(r, P_{E x c}\right) \times h_{\text {det }}(r) .
$$

Figure S3 displays a direct comparison between the simulated PSF and images of a single GaInP segment in a nanowire for four different average excitation powers.

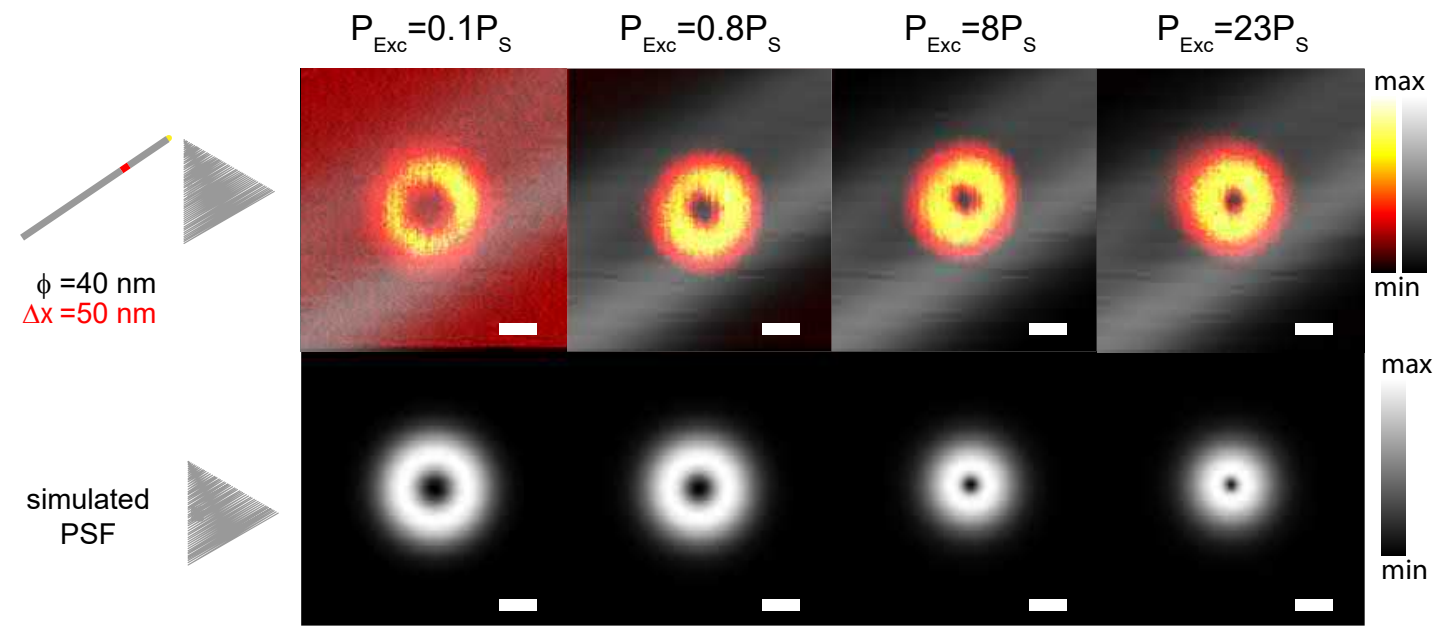

Figure S3. Comparison between raw-data images of a single small luminescent segment with diameter $\phi=40 \mathrm{~nm}$ and length $\Delta x=50 \mathrm{~nm}$ (top) and the predicted PSF of the GSD nanoscope (bottom) for different excitation powers. All scale bars: $200 \mathrm{~nm}$. 


\section{Results}

We compared the GSD nanoscopy results with standard confocal microscopy and scanning electron microscopy (SEM). The tables below report measurements for several NWs in a given field of view. Uncertainties were estimated as the standard deviation of reported measurements. In this study, we defined the contrast as $\left(I_{\max }-I_{\min }\right) /\left(I_{\max }+I_{\min }\right)$.

Notation: $\Delta x_{c}$ - separation of adjacent maxima, $\Delta x$ - apparent segment length (FWHM), $\Delta y-$ apparent segment width (FWHM).

\section{NW \#10188 J3 (Figure 3)}

Table S1. Comparison of segment dimensions for NW \#10188 J3 measured by confocal microscopy, GSD nanoscopy and electron microscopy.

\begin{tabular}{|l|c|c|c|c|c|c|c|c|}
\hline \multirow{2}{*}{ segment } & \multicolumn{2}{|c|}{ Confocal } & \multicolumn{2}{c|}{ GSD raw data } & \multicolumn{2}{c|}{ GSD deconvolved } & \multicolumn{2}{c|}{ SEM } \\
\cline { 2 - 9 } & $\Delta \mathrm{x}(\mathrm{nm})$ & $\Delta \mathrm{y}(\mathrm{nm})$ & $\Delta \mathrm{x}(\mathrm{nm})$ & $\Delta \mathrm{y}(\mathrm{nm})$ & $\Delta \mathrm{x}(\mathrm{nm})$ & $\Delta \mathrm{y}(\mathrm{nm})$ & $\Delta \mathrm{x}(\mathrm{nm})$ & $\Delta \mathrm{y}(\mathrm{nm})$ \\
\hline $\mathrm{GaInP}$ & - & $388 \pm 25$ & $225 \pm 14$ & $76 \pm 11$ & $212 \pm 22$ & $73 \pm 9$ & $233 \pm 39$ & $25 \pm 1$ \\
\hline $\mathrm{GaP}$ & - & & $162 \pm 20$ & & $174 \pm 17$ & & $164 \pm 21$ & $25 \pm 1$ \\
\hline
\end{tabular}

Contrast along NW axis (at the middle segments): $0.13 \pm 0.01$

Contrast across NW axis: $0.10 \pm 0.02$

NW \#9609 D3 (Figure 4)

Table S2. Comparison of segment dimensions for NW \#9609 D3 measured by GSD nanoscopy and electron microscopy.

\begin{tabular}{|l|c|c|c|c|c|c|c|c|}
\hline \multirow{2}{*}{ segment } & \multicolumn{2}{|c|}{ GSD raw data } & \multicolumn{3}{|c|}{ GSD deconvolved data } & \multicolumn{3}{c|}{ SEM } \\
\cline { 2 - 9 } & $\Delta \mathrm{x}_{\mathrm{c}}(\mathrm{nm})$ & $\Delta \mathrm{y}(\mathrm{nm})$ & $\Delta \mathrm{x}_{\mathrm{c}}(\mathrm{nm})$ & $\Delta \mathrm{x}(\mathrm{nm})$ & $\Delta \mathrm{y}(\mathrm{nm})$ & $\Delta \mathrm{x}_{\mathrm{c}}(\mathrm{nm})$ & $\Delta \mathrm{x}(\mathrm{nm})$ & $\Delta \mathrm{y}(\mathrm{nm})$ \\
\hline GaInP & $112 \pm 13$ & $84 \pm 12$ & $116 \pm 6$ & $66 \pm 10$ & $66 \pm 6$ & $114 \pm 5$ & $64 \pm 15$ & $43 \pm 3$ \\
\hline
\end{tabular}

Contrast along NW axis (at the middle segments): $0.10 \pm 0.03$

Contrast across NW axis: $0.21 \pm 0.02$ to $0.07 \pm 0.03$

\section{NW \#9607 A3 (Figure 5)}

Table S3. Comparison of segment dimensions for NW \#9607 A3 measured by GSD nanoscopy and electron microscopy.

\begin{tabular}{|l|c|c|c|c|c|c|c|c|c|}
\hline \multirow{3}{*}{ segment } & \multicolumn{3}{|c|}{ GSD raw data } & \multicolumn{3}{c|}{ GSD deconvolved data } & \multicolumn{3}{c|}{ SEM } \\
\cline { 2 - 10 } & $\Delta \mathrm{x}_{\mathrm{c}}(\mathrm{nm})$ & $\Delta \mathrm{x}(\mathrm{nm})$ & $\Delta \mathrm{y}(\mathrm{nm})$ & $\Delta \mathrm{x}_{\mathrm{c}}(\mathrm{nm})$ & $\Delta \mathrm{x}(\mathrm{nm})$ & $\Delta \mathrm{y}(\mathrm{nm})$ & $\Delta \mathrm{x}_{\mathrm{c}}(\mathrm{nm})$ & $\Delta \mathrm{x}(\mathrm{nm})$ & $\Delta \mathrm{y}(\mathrm{nm})$ \\
\hline $\mathrm{GaInP}$ & $147 \pm 14$ & $69 \pm 6$ & $74 \pm 12$ & $146 \pm 8$ & $63 \pm 13$ & $62 \pm 9$ & $145 \pm 4$ & $50 \pm 9$ & $53 \pm 2$ \\
\hline
\end{tabular}

Contrast along NW axis (at the middle segments): $0.14 \pm 0.01$

Contrast across NW axis: $0.17 \pm 0.04$ 
NW \#10476 B1 (Figure S2)

Table S4. Separation of adjacent maxima and segment dimensions, as measured by GSD nanoscopy for NW \#10476 B1.

\begin{tabular}{|l|l|l|l|l|}
\hline \multicolumn{5}{|c|}{ GSD raw data } \\
\cline { 1 - 4 } segment & $\Delta \mathrm{x}_{\mathrm{c}}$ & segment & $\Delta \mathrm{x}(\mathrm{nm})$ & $\Delta \mathrm{y}(\mathrm{nm})$ \\
\hline $1^{\text {st }}-2^{\text {nd }} \mathrm{GaInP}$ & $164 \pm 9$ & $1^{\text {st }} \mathrm{GaInP}$ & $72 \pm 10$ & \multirow{2}{*}{$67 \pm 12$} \\
\cline { 1 - 4 } $2^{\text {nd }}-3^{\text {rd }} \mathrm{GaInP}$ & $181 \pm 6$ & $2^{\text {nd }} \mathrm{GaInP}$ & $74 \pm 12$ & \\
\cline { 1 - 4 } $3^{\text {rd }}-4^{\text {th }} \mathrm{GaInP}$ & $184 \pm 8$ & $3^{\text {rd }} \mathrm{GaInP}$ & $73 \pm 19$ & \\
\hline & & $4^{\text {th }} \mathrm{GaInP}$ & $85 \pm 24$ & \\
\hline
\end{tabular}

\section{Limitations of direct subdiffraction imaging with GSD (resolution and contrast)}

The resolution enhancement is limited by several factors. One of them is the quality of the doughnut zero, which can influence the image contrast. We found that the non-zero intensity at the minimum (in our case $\zeta=0.001)$ only slightly influenced the contrast at applicable power levels $(<20 \%$ reduction). The predicted resolution and contrast for a point-like luminescent segment $\left(1 \times 1 \mathrm{~nm}^{2}\right)$, calculated for the saturation properties and the analytical PSF (both informed by measurement), is presented in Figure S4.

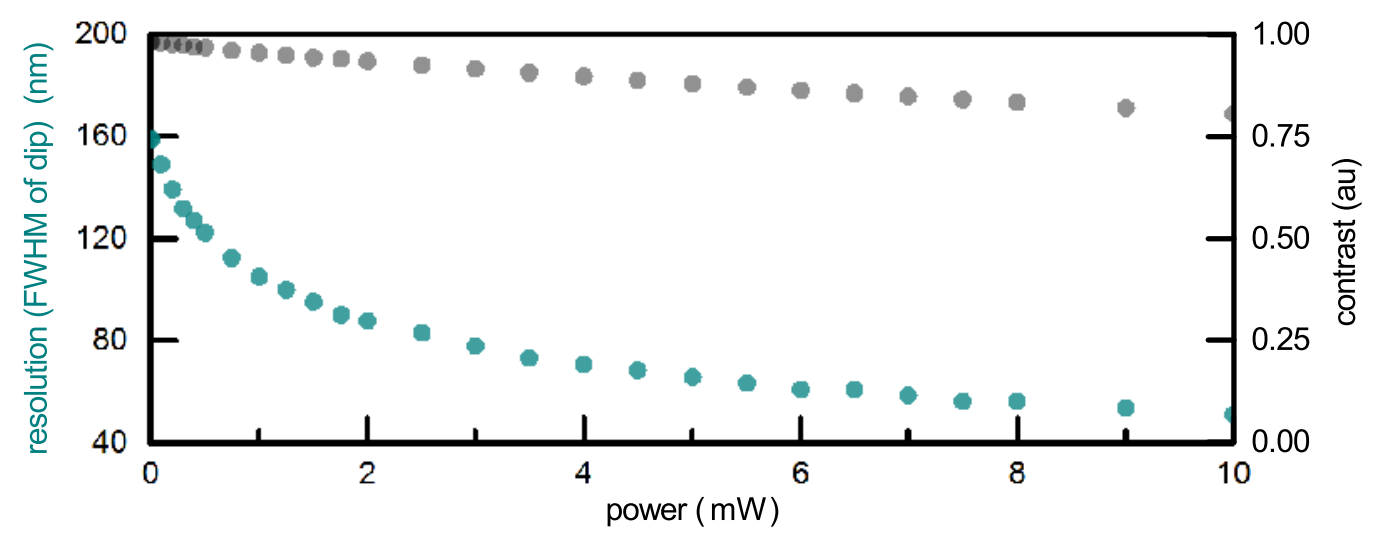

Figure S4. Resolution and contrast simulated for a point-like photoluminescent segment $\left(1 \times 1 \mathrm{~nm}^{2}\right)$. The quality of the doughnut zero was $\zeta=0.001$.

A $66 \mathrm{~nm}$ spatial resolution can be obtained for excitation power $P_{E x c}=5 \mathrm{~mW}$ with meager contrast reduction by $10 \%$. However, significant reductions of the contrast are related to the finite 
size of the PL segments and/or multiple segments spaced within the diffraction limit (Figure 4h,i). In this case, PL of nearby excitons lowers the registered intensity dip. In Figure 4h, we show the contrast reduction as a function of PL segment length for different nanowire diameters $\phi$ (excitation power $5 \mathrm{~mW}$ ). For a single luminescent segment with $20 \mathrm{~nm}$ diameter, the image contrast was reduced from 0.8 to 0.2 by increasing the GaInP length from $20 \mathrm{~nm}$ to $200 \mathrm{~nm}$. Not only the segment length but also the spacing between several emissive segments influenced the depth of observed dips. The contrast as function of the separation (spacer length) between three adjacent luminescent segments with diameter and length both equal $20 \mathrm{~nm}$ is shown in Figure 4i. Separation smaller than the resolution $(\sim 60 \mathrm{~nm})$ leads to indistinguishable segments and was not taken into account. For a separation close to the resolution, the contrast (simulated) has the lowest value $\sim 0.16$; increases in separation lead to nearly linearly increased contrast (Figure 4i).

The highest contrast can be obtained for the smallest luminescent objects at the expense of signal (which scales as the volume, i.e., number of electrons). As nanowires are exceptionally bright, we decreased both the diameter and the length of luminescent segments. However, small NWs (diameter $<20 \mathrm{~nm}$ ) exhibited strong PL intermittency, which makes GSD imaging impracticable (Figure S5). When NW luminescent segment size became comparable to the (bulk) exciton radius (for a $\mathrm{Ga}_{\mathrm{x}} \mathrm{In}_{1-\mathrm{x}} \mathrm{P}$ exciton the Bohr radius is $\sim 4.2-8.6 \mathrm{~nm}$ ), we observed significant fluctuations in emitted PL. Currently, these fluctuations are most often explained as induced by light charging of the semiconductor material, which then cannot emit light. Unneutralized charge is created by photoexcited carriers trapped in acceptor-like surface states, and eventually recombines via a nonradiative Auger-like mechanism. This charging effectively quenches emission until the semiconductor is re-neutralized. The nature of PL fluctuations is still not fully understood ${ }^{4}$. 

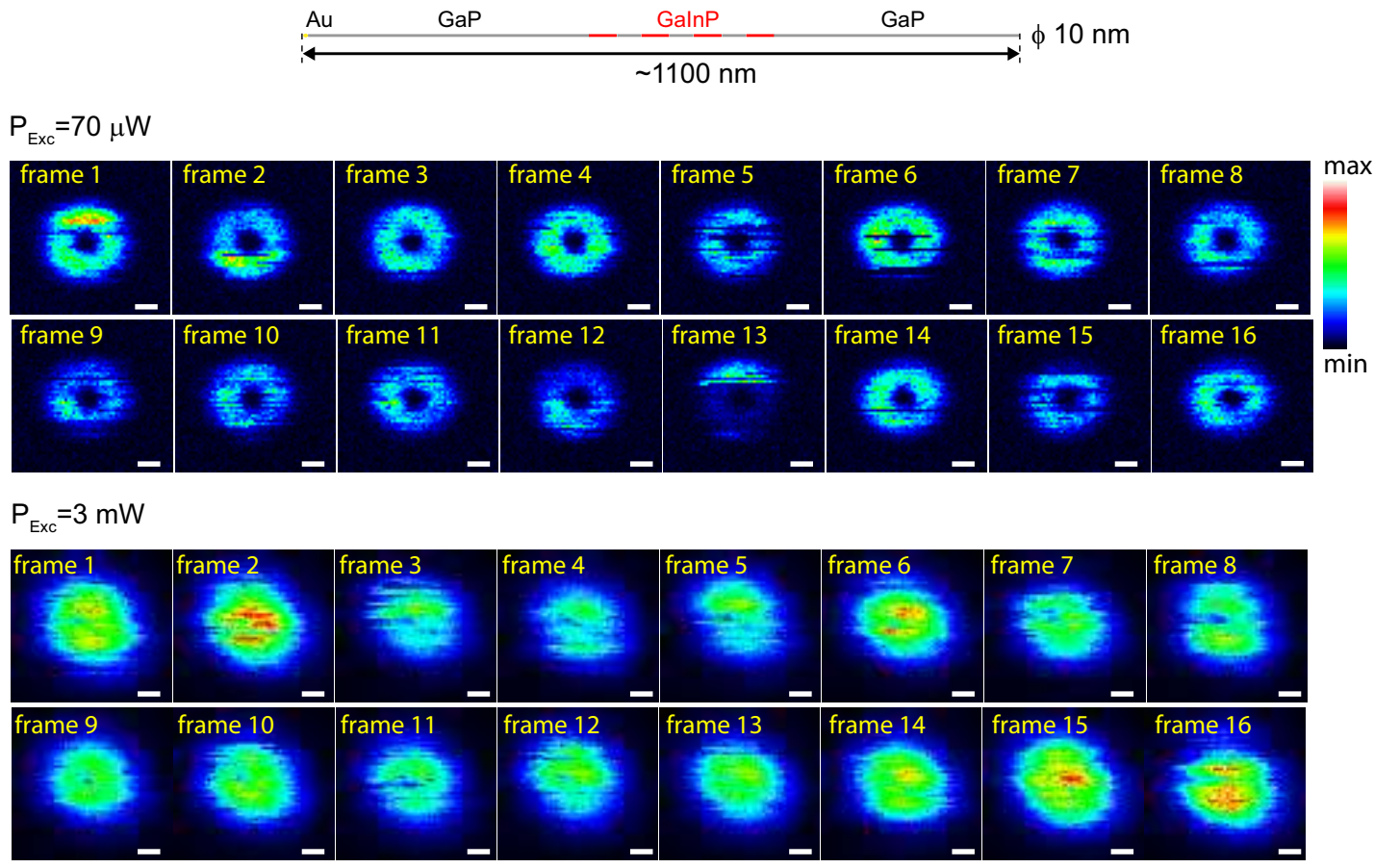

Figure S5. Photoluminescence of a single NW ( $\phi=10 \mathrm{~nm})$ with four PL segments. An increased photoluminescence intermittency was observed due to the small diameter of the NW. The excitation power was $70 \mu \mathrm{W}$ (top) and $3000 \mu \mathrm{W}$ (bottom), the dwell time $1 \mathrm{~ms}$. The photoluminescence segments became indistinguishable due to the strong signal fluctuations, which masked the expected signal dips. All scale bars: $200 \mathrm{~nm}$.

A practical limit of resolution enhancement is related to permanent loss of PL from the emitter structure (Figure S6). Such damage eventually occurs after applying a high-power excitation beam and manifests as significantly lower PL signal and, very often, suddenly increased contrast of the recorded image. This contrast increase is likely due to reduction of the emissive segment volume. This observation is supported by changes in the scattering profile before and after photoluminescence decrease (Figure S6d). For a single NW, the threshold for permanent signal loss varied from 3 to $7 \mathrm{~mW}$, depending on the NWs. The mechanisms of PL decrease are beyond the scope of this article. 
(a)
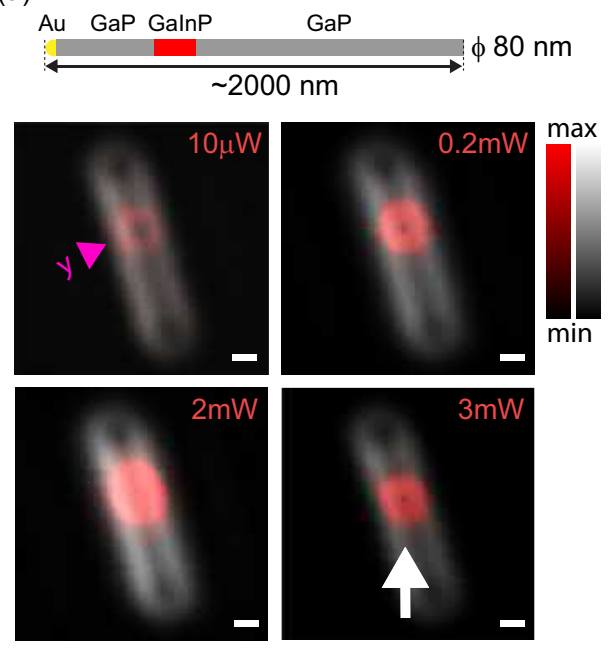

(b)

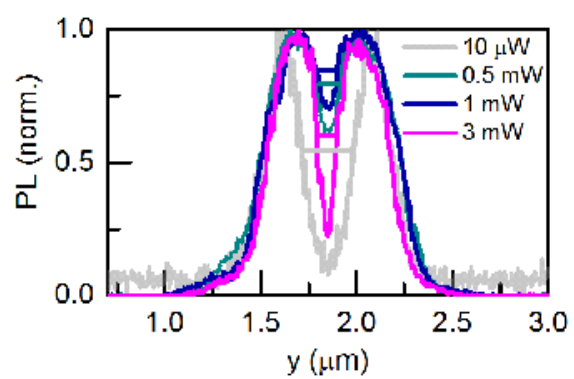

(c)

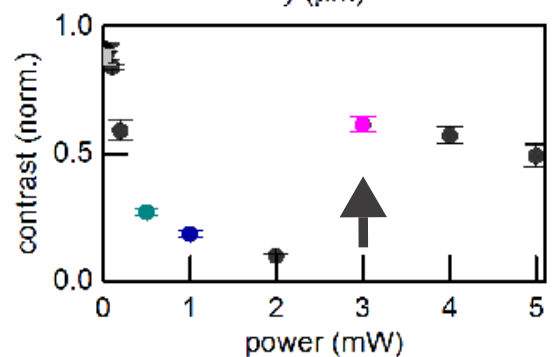

(d)
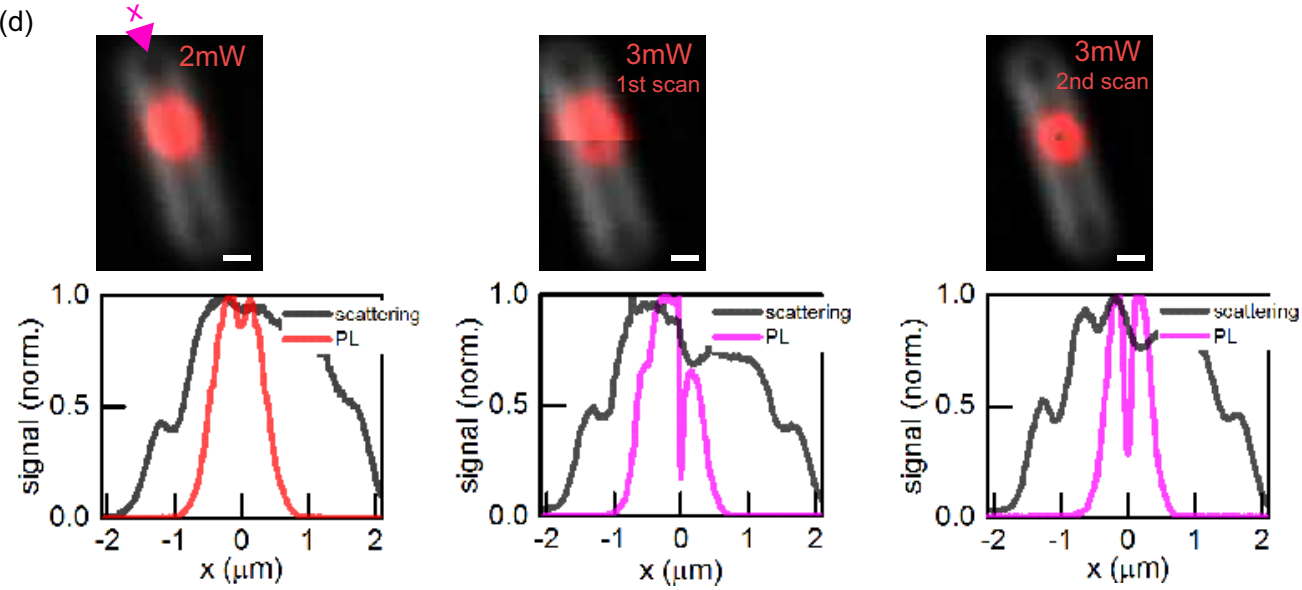

Figure S6. (a) Scattering (gray) and photoluminescence (red) of a single NW ( $\phi=80 \mathrm{~nm})$ with a single GaInP segment. (b) Photoluminescence intensity profiles show resolution changes as a function of the excitation power for the same NW. The resolution was defined as the full-width at half-maximum (FWHM) of the signal dip to minimum. (c) Contrast for data in b. The arrow indicates the abrupt reduction of photoluminescence. (d) The same NW imaged with GSD (successive scans). The decrease in photoluminescence was accompanied by a significant increase in the contrast of the image, which suggests a volume reduction of the luminescent segment. All scale bars: $400 \mathrm{~nm}$. 


\section{Specifications of NWs}

NW \#9030 A8 (Figure 2, Figure S3)

Table S5. Geometry of NW \#9030 A8 based on the growth time.

\begin{tabular}{|l|l|l|l|l|l|}
\hline \multirow{2}{*}{$\begin{array}{l}\text { NW } \\
\text { density }\end{array}$} & \multirow{2}{*}{$\begin{array}{l}\text { gold } \\
\text { bead }\end{array}$} & \multicolumn{3}{|c|}{ growth properties } & \multirow{2}{*}{ total length } \\
\cline { 3 - 5 } & & $2 \mathrm{~min}$ & $5 \mathrm{~s}$ & $2 \mathrm{~min}$ & \\
\cline { 3 - 5 } & $\mathrm{GaP}$ & $\mathrm{GaInP}$ & $\mathrm{GaP}$ & \\
\hline $4 / \mu \mathrm{m}^{2}$ & $40 \mathrm{~nm}$ & $\sim 1.1 \mu \mathrm{m}$ & $<100 \mathrm{~nm}$ & $\sim 0.9 \mu \mathrm{m}$ & $\sim 2 \mu \mathrm{m}$ \\
\hline
\end{tabular}

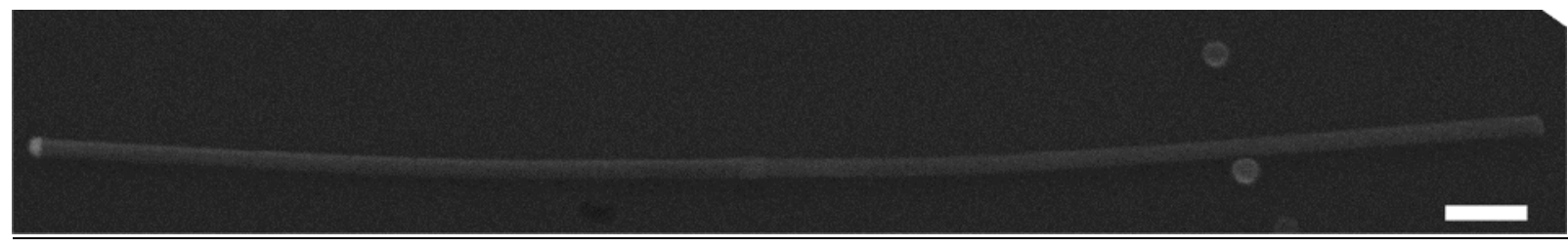

Figure S7. Example SEM image of barcode NW \#9030 A8. Measured GaInP segment length and width both $\sim 50 \mathrm{~nm}$. Scale bar: $200 \mathrm{~nm}$.

NW \#10188 J3 (Figure 3)

Table S6. Geometry of NW \#10188 J3 deduced from growth time.

\begin{tabular}{|l|l|l|l|l|l|l|l|l|l|l|l|}
\hline \multirow{2}{*}{$\begin{array}{l}\text { NW } \\
\text { density }\end{array}$} & \multirow{2}{*}{$\begin{array}{l}\text { gold } \\
\text { bead }\end{array}$} & \multicolumn{9}{|c|}{ growth properties } & total \\
& & $2 \mathrm{~min}$ & $5 \mathrm{~s}$ & $10 \mathrm{~s}$ & $5 \mathrm{~s}$ & $10 \mathrm{~s}$ & $5 \mathrm{~s}$ & $10 \mathrm{~s}$ & $5 \mathrm{~s}$ & $1 \mathrm{~min}$ & length \\
\cline { 3 - 12 } & & $\mathrm{GaP}$ & $\mathrm{GaInP}$ & $\mathrm{GaP}$ & GaInP & GaP & GaInP & GaP & GaInP & GaP \\
\hline $2 / \mu \mathrm{m}^{2}$ & $20 \mathrm{~nm}$ & $\sim 1.4 \mu \mathrm{m}$ & $\sim 150 \mathrm{~nm}$ & $\sim 100 \mathrm{~nm}$ & $\sim 150 \mathrm{~nm}$ & $\sim 100 \mathrm{~nm}$ & $\sim 150 \mathrm{~nm}$ & $\sim 100 \mathrm{~nm}$ & $\sim 150 \mathrm{~nm}$ & $\sim 1.2 \mu \mathrm{m}$ & $\sim 3.5 \mu \mathrm{m}$ \\
\hline
\end{tabular}

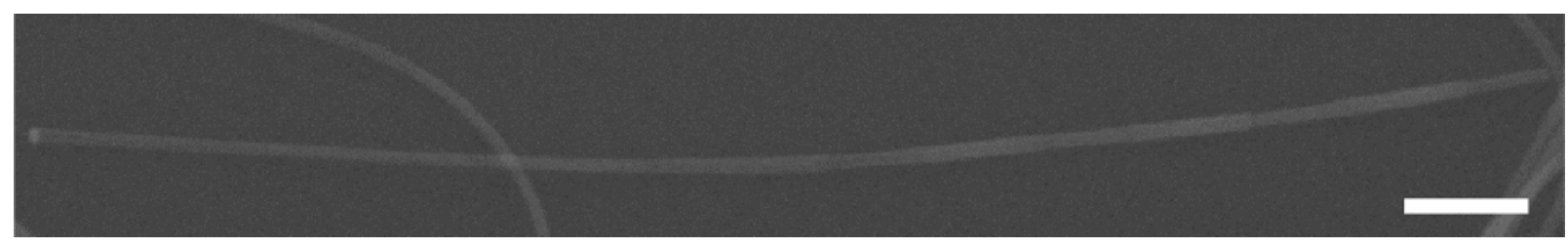

Figure S8. Example SEM image of barcode NW \#10188 J3. Measured GaInP/GaP segment lengths equal $233 \pm 39 / 164 \pm 21 \mathrm{~nm}$ respectively, with diameter $25 \pm 2 \mathrm{~nm}$. Scale bar: $200 \mathrm{~nm}$.

\section{NW \#9609 D3 (Figure 4)}

Table S7. Geometry of NW \#9609 D3 deduced from growth time.

\begin{tabular}{|c|c|c|c|c|c|c|c|c|c|c|c|}
\hline \multirow{3}{*}{$\begin{array}{l}\text { NW } \\
\text { density }\end{array}$} & \multirow{3}{*}{$\begin{array}{l}\text { gold } \\
\text { bead } \\
\phi\end{array}$} & \multicolumn{9}{|c|}{ growth properties } & \multirow{3}{*}{$\begin{array}{l}\text { total } \\
\text { length }\end{array}$} \\
\hline & & $2 \min$ & $5 \mathrm{~s}$ & $7 \mathrm{~s}$ & $5 \mathrm{~s}$ & $7 \mathrm{~s}$ & $5 \mathrm{~s}$ & $7 \mathrm{~s}$ & $5 \mathrm{~s}$ & $1 \min$ & \\
\hline & & $\mathrm{GaP}$ & GaInP & $\mathrm{GaP}$ & GaInP & $\mathrm{GaP}$ & GaInP & $\mathrm{GaP}$ & GaInP & $\mathrm{GaP}$ & \\
\hline $2 / \mu \mathrm{m}^{2}$ & $40 \mathrm{~nm}$ & $\sim 0.4 \mu \mathrm{m}$ & $\sim 70 \mathrm{~nm}$ & $\sim 70 \mathrm{~nm}$ & $\sim 70 \mathrm{~nm}$ & $\sim 70 \mathrm{~nm}$ & $\sim 70 \mathrm{~nm}$ & $\sim 70 \mathrm{~nm}$ & $\sim 70 \mathrm{~nm}$ & $\sim 0.5 \mu \mathrm{m}$ & $\sim 1.4 \mu \mathrm{m}$ \\
\hline
\end{tabular}




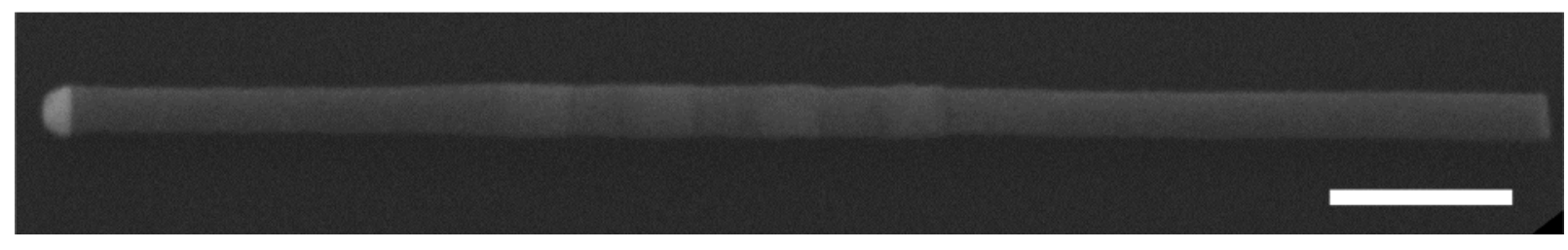

Figure S9. Example SEM image of barcode NW \#9609 D3. Measured GaInP segment length $64 \pm 15 \mathrm{~nm}$, with $114 \pm 5 \mathrm{~nm}$ separation between the adjacent maxima. NW diameter retrieved by SEM: $43 \pm 3 \mathrm{~nm}$. Scale bar: $200 \mathrm{~nm}$.

\section{NW \#9607 A3 (Figure 5)}

Table S8. Geometry of NW \#9607 A3 deduced from growth time.

\begin{tabular}{|c|c|c|c|c|c|c|c|c|c|c|c|}
\hline \multirow{3}{*}{$\begin{array}{l}\text { NW } \\
\text { density }\end{array}$} & \multirow{3}{*}{$\begin{array}{l}\text { gold } \\
\text { bead } \\
\phi\end{array}$} & \multicolumn{9}{|c|}{ growth properties } & \multirow{3}{*}{$\begin{array}{l}\text { total } \\
\text { length }\end{array}$} \\
\hline & & $2 \min$ & $5 \mathrm{~s}$ & $10 \mathrm{~s}$ & $5 \mathrm{~s}$ & $10 \mathrm{~s}$ & $5 \mathrm{~s}$ & $10 \mathrm{~s}$ & $5 \mathrm{~s}$ & $1 \mathrm{~min}$ & \\
\hline & & $\mathrm{GaP}$ & GaInP & $\mathrm{GaP}$ & GaInP & $\mathrm{GaP}$ & GaInP & $\mathrm{GaP}$ & GaInP & $\mathrm{GaP}$ & \\
\hline $2 / \mu \mathrm{m}^{2}$ & $40 \mathrm{~nm}$ & $\sim 500 \mathrm{~nm}$ & $\sim 50 \mathrm{~nm}$ & $\sim 0.1 \mu \mathrm{m}$ & $\sim 50 \mathrm{~nm}$ & $\sim 0.1 \mu \mathrm{m}$ & $\sim 50 \mathrm{~nm}$ & $\sim 0.1 \mu \mathrm{m}$ & $\sim 50 \mathrm{~nm}$ & $\sim 500 \mathrm{~nm}$ & $\sim 1.5 \mu \mathrm{m}$ \\
\hline
\end{tabular}

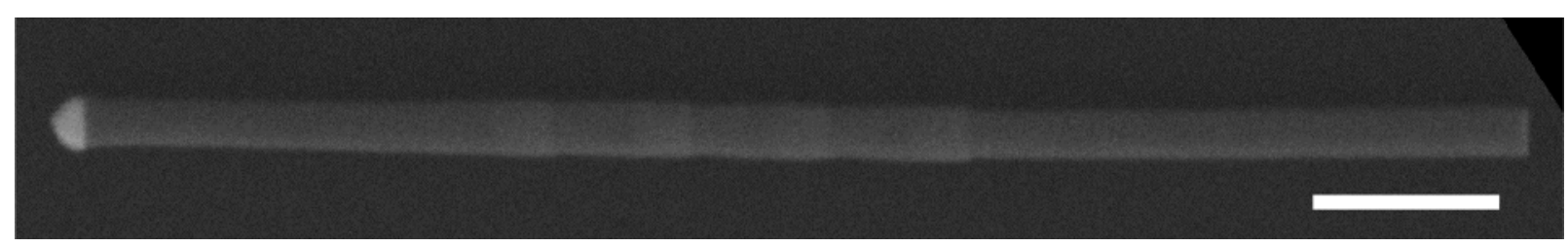

Figure S10. Example SEM image of barcode nanowire \#9607 A3. Measured GaInP segment length $50 \pm 9 \mathrm{~nm}$, with $145 \pm 4 \mathrm{~nm}$ separation between the adjacent maxima. NW diameter retrieved by SEM: $53 \pm 2 \mathrm{~nm}$. Scale bar: $200 \mathrm{~nm}$.

\section{NW \#10476 B1 (Figure S2)}

Table S9. Geometry of NW \#10476 B1 deduced from growth time.

\begin{tabular}{|l|l|l|l|l|l|l|l|l|l|l|l|}
\hline \multirow{2}{*}{$\begin{array}{l}\text { NW } \\
\text { density }\end{array}$} & $\begin{array}{l}\text { gold } \\
\text { bead } \\
\end{array}$ & \multicolumn{9}{|c|}{ growth properties } & total \\
& $\phi$ & $2 \mathrm{~min}$ & $5 \mathrm{~s}$ & $12 \mathrm{~s}$ & $5 \mathrm{~s}$ & $10 \mathrm{~s}$ & $5 \mathrm{~s}$ & $8 \mathrm{~s}$ & $5 \mathrm{~s}$ & $1 \mathrm{~min}$ & length \\
\cline { 3 - 13 } & $\mathrm{GaP}$ & GaInP & GaP & GaInP & GaP & GaInP & GaP & GaInP & GaP & \\
\hline $2 / \mu \mathrm{m}^{2}$ & $20 \mathrm{~nm}$ & $\sim 500 \mathrm{~nm}$ & $\sim 50 \mathrm{~nm}$ & $\sim 90 \mathrm{~nm}$ & $\sim 50 \mathrm{~nm}$ & $\sim 90 \mathrm{~nm}$ & $\sim 50 \mathrm{~nm}$ & $\sim 90 \mathrm{~nm}$ & $\sim 50 \mathrm{~nm}$ & $\sim 600 \mathrm{~nm}$ & $\sim 1.6 \mu \mathrm{m}$ \\
\hline
\end{tabular}

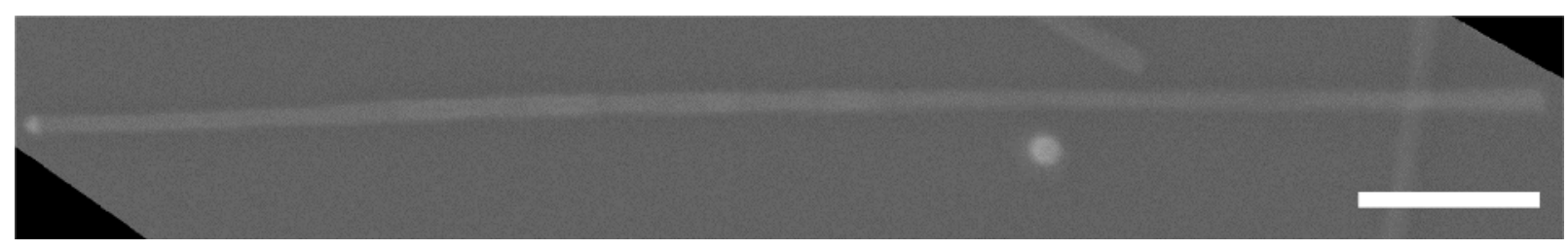

Figure S11. SEM image of barcode nanowire \#10476 B1. Scale bar: $200 \mathrm{~nm}$. 
NW \#10474 A6 (Figure S5)

Table S10. Geometry of NW \#10474 with growth times for individual segments.

\begin{tabular}{|c|c|c|c|c|c|c|c|c|c|c|c|}
\hline \multirow{3}{*}{$\begin{array}{l}\mathrm{NW} \\
\text { density }\end{array}$} & \multirow{3}{*}{$\begin{array}{l}\text { gold } \\
\text { bead } \\
\phi\end{array}$} & \multicolumn{9}{|c|}{ growth properties } & \multirow[t]{3}{*}{ total length } \\
\hline & & $2 \mathrm{~min}$ & $5 \mathrm{~s}$ & $10 \mathrm{~s}$ & $5 \mathrm{~s}$ & $8 \mathrm{~s}$ & $5 \mathrm{~s}$ & $5 \mathrm{~s}$ & $5 \mathrm{~s}$ & $1 \mathrm{~min}$ & \\
\hline & & $\mathrm{GaP}$ & GaInP & $\mathrm{GaP}$ & GaInP & $\mathrm{GaP}$ & GaInP & $\mathrm{GaP}$ & GaInP & $\mathrm{GaP}$ & \\
\hline $4 / \mu \mathrm{m}^{2}$ & $10 \mathrm{~nm}$ & $*$ & $*$ & * & * & $*$ & $*$ & $*$ & $*$ & * & $\sim 11 \mathrm{um}$ \\
\hline
\end{tabular}

(*) difficult to estimate segment lengths

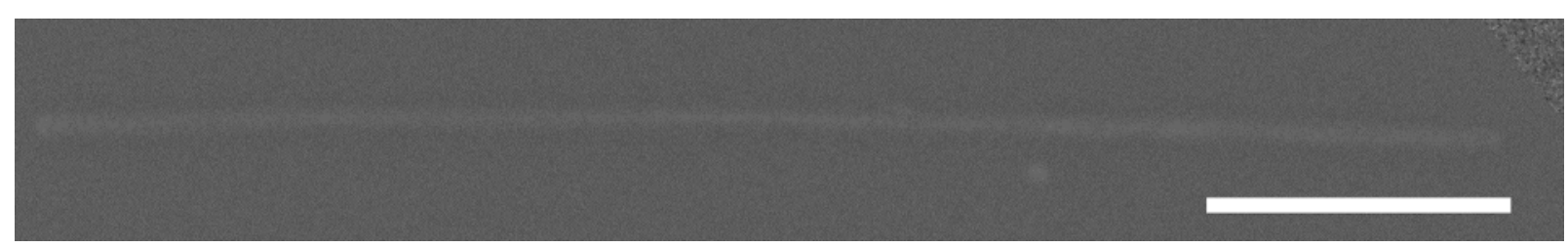

Figure S12. SEM image of barcode nanowire \#10474 A6. Scale bar: $200 \mathrm{~nm}$.

NW \#7816 (Figure S6)

Table S11. Geometry of NW \#7816 deduced from growth time.

\begin{tabular}{|l|l|l|l|l|l|}
\hline \multirow{2}{*}{$\begin{array}{l}\text { NW } \\
\text { density }\end{array}$} & \multirow{2}{*}{$\begin{array}{l}\text { gold } \\
\text { bead }\end{array}$} & \multicolumn{3}{|c|}{ growth properties } & \multirow{2}{*}{ total length } \\
\cline { 3 - 5 } & $\phi$ & $3 \mathrm{~min}$ & $1+15 \mathrm{~s}$ & $1.5 \mathrm{~min}$ & \\
\cline { 3 - 5 } & $\mathrm{GaP}$ & $\mathrm{GaInP}$ & $\mathrm{GaP}$ & \\
\hline $2 / \mu \mathrm{m}^{2}$ & $80 \mathrm{~nm}$ & $\sim 1.3 \mu \mathrm{m}$ & $\sim 200 \mathrm{~nm}$ & $\sim 0.5 \mu \mathrm{m}$ & $\sim 2 \mu \mathrm{m}$ \\
\hline
\end{tabular}

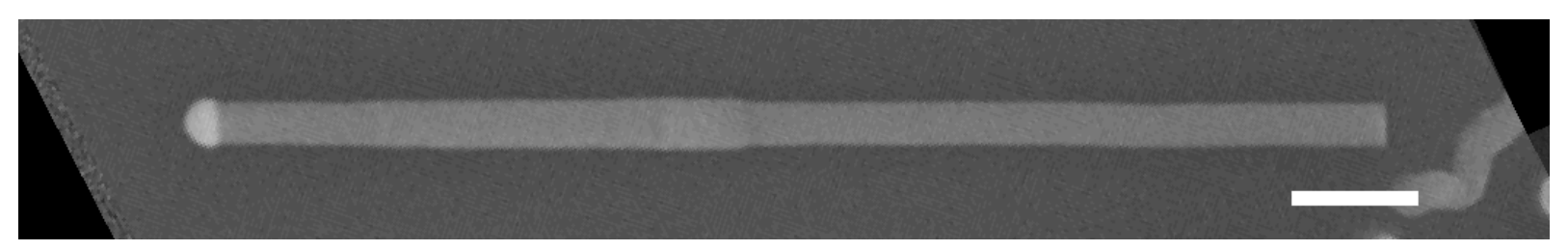

Figure S13. SEM image of barcode nanowire \#7816. Scale bar: $200 \mathrm{~nm}$.

\section{Lookup tables (color scales)}

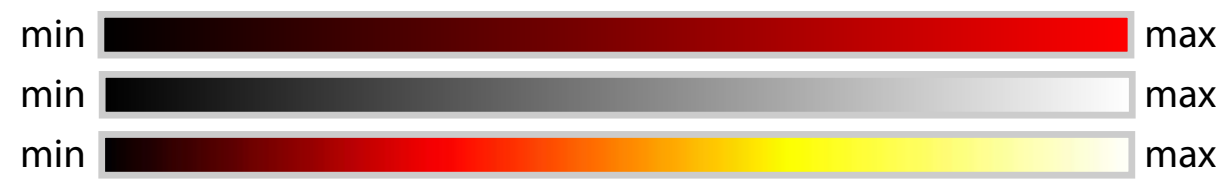

Figure S14. Color scales used in Figures 2-5. 


\section{References}

1. $\quad$ Wagner, R. S.; Ellis, W. C. Appl. Phys. Lett. 1964, 4, (5), 89-90.

2. Magnusson, M. H.; Deppert, K.; Malm, J.-O.; Bovin, J.-O.; Samuelson, L. Nanostruct. Mater. 1999, 12, (1-4), 45-48.

3. $\quad$ Rittweger, E.; Wildanger, D.; Hell, S. W. EPL 2009, 86, (1), 14001.

4. Frantsuzov, P.; Kuno, M.; Janko, B.; Marcus, R. A. Nat. Phys. 2008, 4, (5), 519-522. 



\section{Acknowledgments}

Scientific research is a collaborative effort and I would like to express my gratitude to many people who had an influence on me and on the final shape of this thesis.

First of all, I would like sincerely thank to Prof. Stefan W. Hell, who not only fully supported my research and allowed me to freely perform experiments in his well-equipped lab, but most importantly who always had time to discuss science. His unique way of addressing scientific problems was a great source of inspiration for me.

Secondly, I want to express my gratitude to Prof. Czesław Radzewicz, thanks to whom I directed my interest towards optics in general, and later particularly to super-resolution microscopy. I am very grateful for the opportunity to work in his Ultrafast Phenomena Laboratory from the beginning of my studies at the University of Warsaw. I will never forget the faith he had in me, allowing me to realize my own sonoluminescence project (which at the end was a failure), his patience and support during my early years in his laboratory, and his boundless knowledge, which he was always happy to share.

I would like to thank to Prof. Tim Salditt for his interest in my work and his willingness to be the advisor of my thesis.

Many thanks to Dr. Steffen J. Sahl, who notably influenced all of my manuscripts and also my development as a scientist. Steffen, I highly appreciate our discussions, your help and advise. I am very grateful for the opportunity to learn from you. I want to express my gratitude also to Dr. Volker Westphal for his support. Volker, it has been a pleasure to work with you.

I would like to thank Dr. Karl Adolfsson, the King of nanostructure growth. Kalle, I was very lucky to work with you and I highly appreciate your enthusiasm and knowledge about semiconductor heterostructures. I am also very grateful to Prof. Christelle Prinz for providing the photoluminescent nanowires used in this study, and for many interesting discussions and the opportunity to visit Lund University.

I would like to acknowledge the support of the extraordinary infrastructure at the Max Planck Institute for Biophysical Chemistry, especially its mechanical workshop, the electrical workshop and the optical workshop. It was a pleasure to perform experiments in such an active environment and I have to admit I am quite spoiled now. I am very grateful especially for the technical assistance of Jaydev Jethwa and Rainer Pick.

I want to thank Dr. Carola Gregor and Dr. Jan Keller for their critical reading of large parts of this thesis.

The work in the lab, however pleasant it is, was definitely further enriched by the many people whom I had a chance to know over several years in the Department of NanoBiophotonics. In particular, I would like to thank my friends Tatiana, Carola, Kamila, Mark, Felix, Volker, Silvia, Steffen, Francisco, Martín and Ulrike. Thank you for making this department such a wonderful place to work. 
I also would like to acknowledge my friends from the Faculty of Physics at the University of Warsaw for all the fun in the lab and during preparations of many didactic shows, especially Yuriy, Piotrek Ć., Radek, Filip, Piotr F., Piotr W., Michał K., Michał N., Piotrek M. and Marcin.

I am thankful to my best friends who enhanced the quality of my life in Göttingen. Special thanks to Mish and Dorota. I am very grateful to know you.

Finally, I would like to thank my family for their unlimited support. 


\section{Joanna Oracz - Curriculum vitae}

\section{Personal Information}

E-mail: joanna.oracz@mpibpc.mpg.de

Address: Rote Straße 29, 37073 Göttingen, Germany

Tel.: (+49) 15226773851

Born 23 October 1987 in Katowice, Poland

\section{Education}

2015-2018 University of Göttingen, $\mathrm{PhD}$ programme in physics supervisors: Prof. Czesław Radzewicz, Prof. Stefan W. Hell

2011-2018 University of Warsaw, PhD programme in physics supervisors: Prof. Czesław Radzewicz, Prof. Stefan W. Hell

2006-2011 University of Warsaw, MSc programme in physics; thesis: 'High-resolution fluorescence microscopy' supervisor: Prof. Czesław Radzewicz

\section{Professional experience}

\section{2-present Max Planck Institute for Biophysical Chemistry}

Department of NanoBiophotonics, head: Prof. Stefan W. Hell projects: photobleaching in stimulated emission depletion (STED) microscopy, superresolution microscopy with photoluminescent nanowires

2011 Faculty of Physics, University of Warsaw Ultrafast Phenomena Laboratory, head: Prof. Czesław Radzewicz project: prototype construction of a STED microscope with lateral resolution $<100 \mathrm{~nm}$

2010 Institute of Physical Chemistry, Polish Academy of Sciences Laser Center, head: Prof. Czesław Radzewicz project: construction of adaptive ultrashort pulse shaper based on multiphoton intrapulse interference phase scan (MIIPS)

2006-2007 Faculty of Physics, University of Warsaw

Ultrafast Phenomena Laboratory, head: Prof. Czesław Radzewicz project: experimental setup for real-time pulse characterization based on spatiotemporal spectral interferometry (SEA TADPOLE) 


\section{Publications and articles}

2017 'Photobleaching in STED nanoscopy and its dependence on the photon flux applied for reversible silencing of the fluorophore'

Joanna Oracz, Volker Westphal, Czesław Radzewicz, Steffen J. Sahl and Stefan W. Hell. Scientific Reports 7, 11354 (2017)

2017 'Ground state depletion nanoscopy resolves semiconductor nanowire barcode segments at room temperature'

Joanna Oracz, Karl Adolfsson, Volker Westphal, Czesław Radzewicz, Magnus T. Borgström, Steffen J. Sahl, Christelle N. Prinz, and Stefan W. Hell.

Nano Letters 17 (4), 2652-2659 (2017), (see also Nature Photonics 11, 267 (2017))

2015 'Nagroda Nobla z chemii 2014 - Super-rozdzielcza mikroskopia optyczna wykorzystująca tradycyjne soczewki'

Joanna Oracz

Chemia w Szkole 1/2015; (in Polish)

2012 'Measurement of the mass of an object hanging from a spring-revisited'

Kamil Serafin, Joanna Oracz, Marcin Grzybowski, Maciej Koperski, Paweł Sznajder, Łukasz Zinkiewicz and Piotr Wasylczyk

European Journal of Physics 33, 129-134 (2012)

\section{Teaching experience}

2015/2016 Experimentalphysik I (tutor, in English); Faculty of Physics, University of Göttingen $\sim 30 \mathrm{~h}$ in equivalent of tutorial hours

2014/2015 The Course 'Start' - Physics advanced level (tutor); Faculty of Physics, University of Warsaw $\sim 60 \mathrm{~h}$ in equivalent of tutorial hours

2014/2015 Fundamentals of Physics III (assistant); Faculty of Physics, University of Warsaw $\sim 60 \mathrm{~h}$ in equivalent of tutorial hours

2011/2012 The Course 'Plus' - Physics basic level (tutor); Faculty of Physics, University of Warsaw $\sim 30 \mathrm{~h}$ in equivalent of tutorial hours

2011/2012 Computing workshop (tutor); Faculty of Physics, University of Warsaw $\sim 60 \mathrm{~h}$ in equivalent of tutorial hours

\section{Scientific and didactic activities}

2015 invited talk at Summer School of Optical Microscopy (25-28/06/2015, Rynia, Poland)

2011 inter alia co-founder of University of Warsaw Student Chapter of Optical Society

2009.12 research internship at Institut d'Alembert ENS Cachan, Paris Quantum and Molecular Photonics Laboratory, supervised by Dr. Dominique Chauvat project: far-field imaging of nanocrystals using second-harmonic generation

2007-2008 organizer of the $7^{\text {th }}$ Polish Physics Students' Society's Conference (7-10/11/2008, University of Warsaw)

2007-2009 president of the Physics Students Society at the University of Warsaw

2006-2011 volunteering for many didactic and scientific shows

2006-2011 several talks at students' conferences 


\section{Conference posters}

2014 'Pulse shape dependence of photobleaching in STED microscopy' Joanna Oracz, Volker Westphal, Stefan W. Hell Labeling and Nanoscopy, Heidelberg, Germany

2011 'High-resolution fluorescence microscopy' Joanna Oracz, Czesław Radzewicz $2^{\text {nd }}$ Polish Optical Conference, Szczecin, Poland

\section{Curses attended}

2010 Summer course: quantum engineering (advanced level), Warsaw

2009 Nonlinear photonics and optical telecommunications, Szczecin

\section{Fellowships, awards}

2011-2016 Fellowship of the Foundation for Polish Science; International Ph.D. Projects Programme

$20113^{\text {rd }}$ place in Experimental Confrontations at Faculty of Physics (Faculty of Physics, University of Warsaw), co-author: Marcin J. Grzybowski competition to determine the mass of a metal cylinder hanging on a spring inside a transparent enclosure within $24 \mathrm{~h}$ time limit (results published in European Journal of Physics 33, 129-134 (2012))

$20102^{\text {nd }}$ place in contest Girls of the Future - in the footsteps of Maria Skłodowska-Curie (Ministry of Science and Higher Education of the Republic of Poland, Elle magazine)

$20061^{\text {st }}$ place in the Didactic Show Competition for Students and Doctoral Students of the Faculty of Physics (Faculty of Physics, University of Warsaw) 'Diffraction of light by sound; home-built acusto-optic modulator'

2006 Bronze medal, International Conference of Young Scientists (Heidehof-Stiftung Stuttgart), co-author: Bartłomiej Jańczak 'Physics in sport, that is how to score from the corner?'

2005, $20062^{\text {nd }}$ place in national Young Physicists' Tournament (Polish Physical Society), Creative Group Quark

\section{Additional information}

Languages Native speaker of Polish. Fluent in English.

Programming LabView, MATLAB, Mathematica, C++ 
